
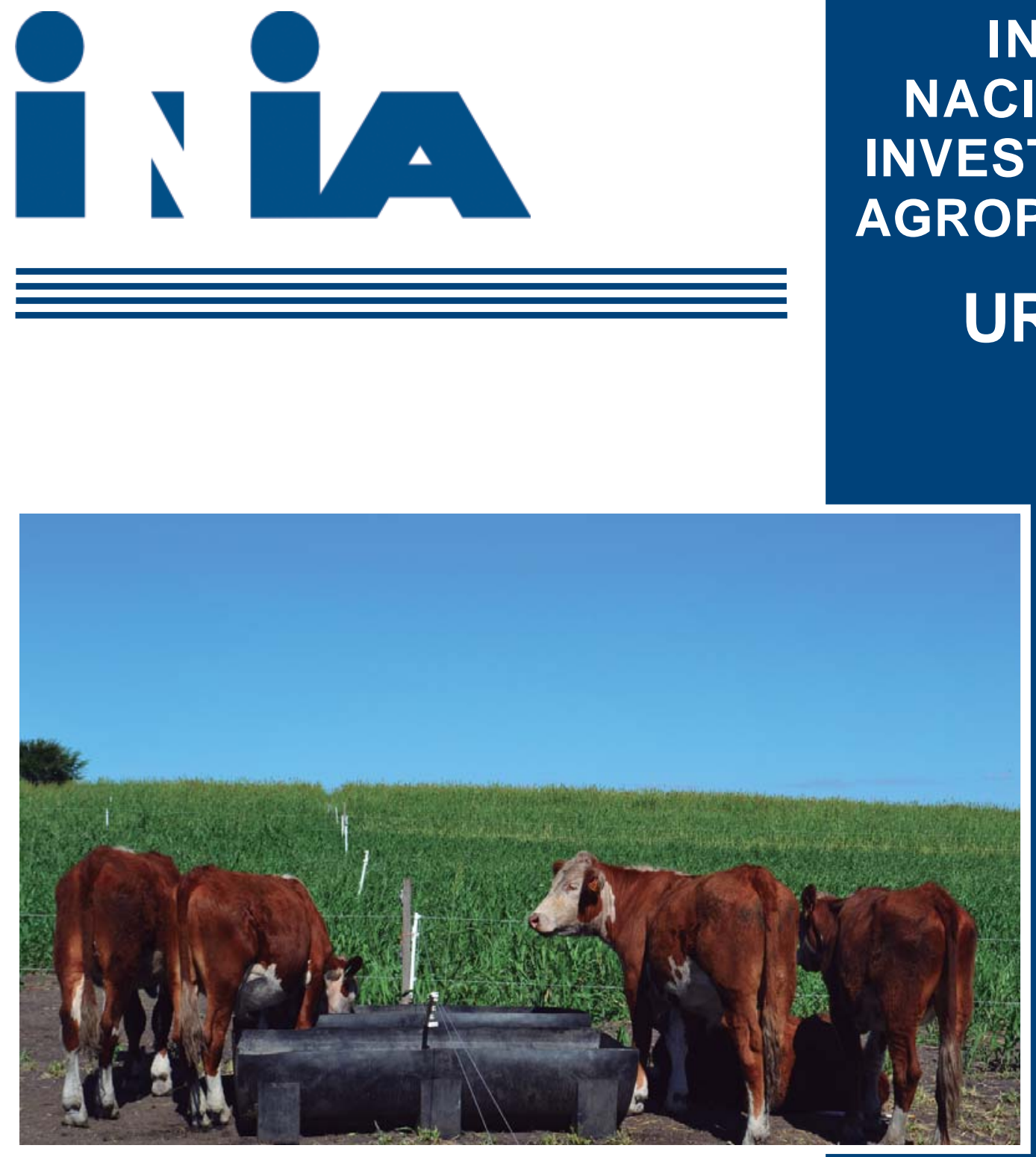

SISTEMAS DE

ENGORDE DE

NOVILLOS SOBRE

VERDEOS ANUALES

ESTIVALES EN LA

REGIÓN DE BASALTO
INSTITUTO NACIONAL DE INVESTIGACIÓN AGROPECUARIA

URUGUAY 


\title{
SISTEMAS DE ENGORDE DE NOVILLOS SOBRE VERDEOS ANUALES ESTIVALES EN LA REGIÓN DE BASALTO
}

\author{
Editores: Montossi, Fabio* \\ Cazzuli, Fiorella* \\ Lagomarsino, Ximena ${ }^{* * *}$
}


Título: SISTEMAS DE ENGORDE DE NOVILLOS SOBRE VERDEOS ANUALES ESTIVALES EN LA REGIÓN DE BASALTO

Editores: Fabio Montossi, Fiorella Cazzuli, Ximena Lagomarsino

Serie Técnica $N^{\circ} 230$

(C) 2017, INIA

ISBN 978-9974-38-375-3

Editado por la Unidad de Comunicación y Transferencia de Tecnología del INIA Andes 1365, Piso 12. Montevideo - Uruguay

http://www.inia.uy

Quedan reservados todos los derechos de la presente edición. Esta publicación no se podrá reproducir total o parcialmente sin expreso consentimiento del INIA. 


\section{I nstituto Nacional de I nvestigación Agropecuaria}

\section{Integración de la Junta Directiva}

Ing. Agr., MSc., PhD. Álvaro Roel - Presidente

D.M.T.V., PhD. J osé Luis Repetto - Vicepresidente

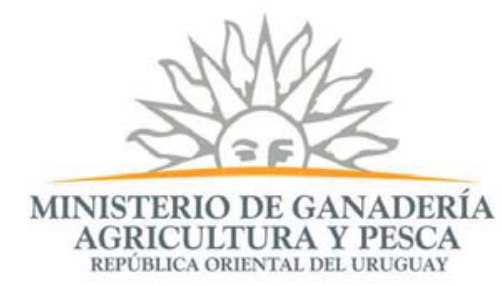

Ing. Agr. MSc., Diego Payssé Salgado

Ing. Agr. J orge Peñagaricano
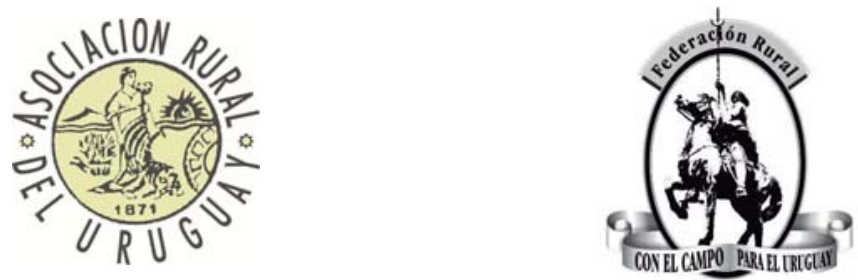

Ing. Agr. Pablo Gorriti

Ing. Agr. Alberto Bozzo
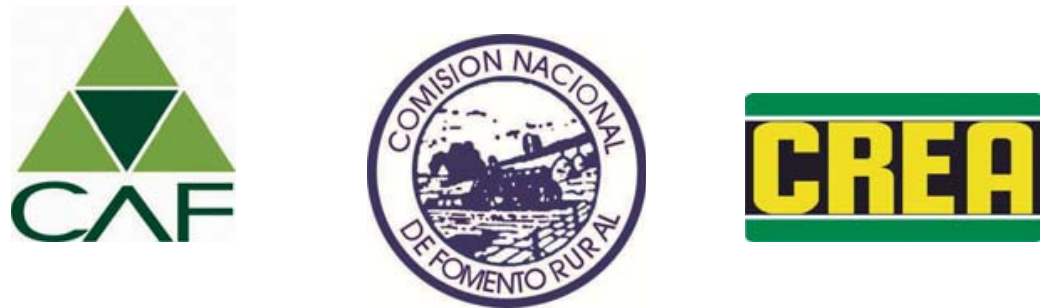


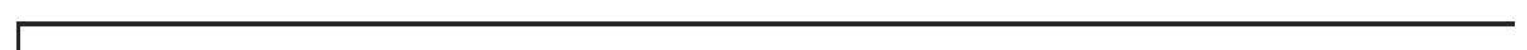




\section{CONTENIDO}

Página

I. INTRODUCCIÓN .................................................................................................. 1

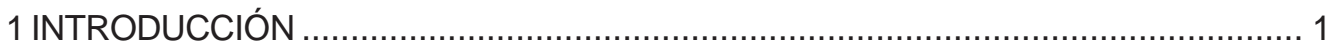

1.1. Contexto de la producción, faena y mercado de carne vacuna de la Región de Basalto y del Uruguay

1.2. Contexto y justificación de la propuesta tecnológica desarrollada en esta publicación

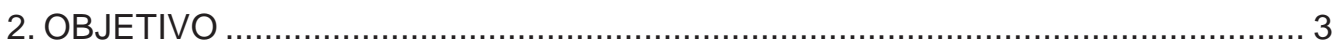

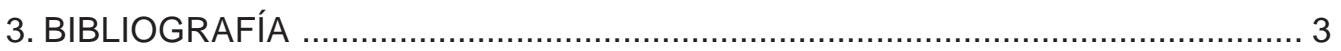

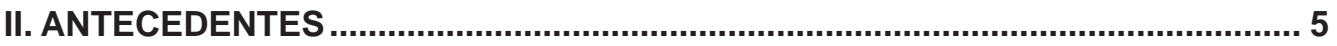

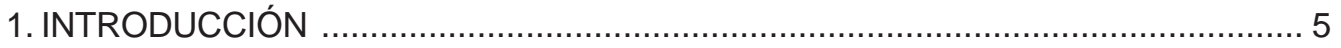

2. SORGOS FORRAJEROS (SORGHUM SP.) …......................................... 3

2.1. Sudangrás (Sorghum sudanense) ...................................................... 6

2.2. Sorgos híbridos (Sorghum $x$ S. sudanense) …......................................... 7

3. SUPLEMENTACIÓN DE BOVINOS SOBRE VERDEOS DE VERANO ................. 8

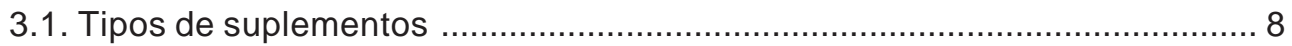

4. PERFORMANCE ANIMAL DE NOVILLOS SOBRE VERDEOS DE VERANO ...... 9

4.1. Requerimientos de energía y proteína ..................................................... 9

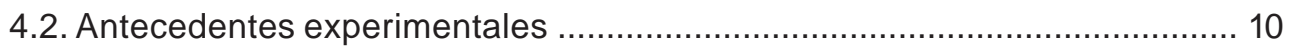

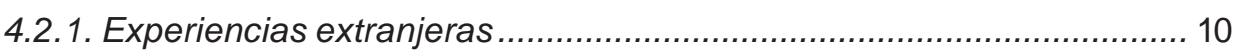

4.2.2 .Experiencias nacionales .............................................................. 15

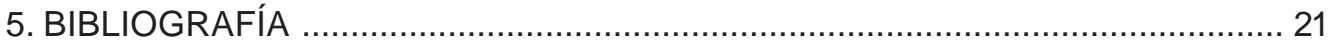

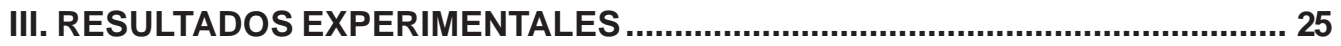

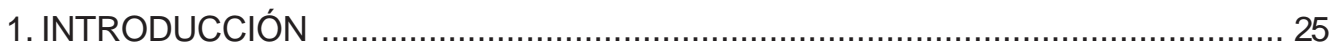

2. EVALUACIÓN DE LA PRODUCTIVIDAD DE NOVILLOS PASTOREANDO

SORGO FORRAJERO Y SUDANGRÁS CON SUPLEMENTACIÓN PROTEICA . 25

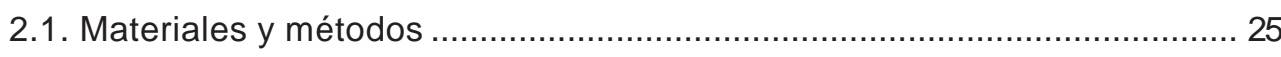

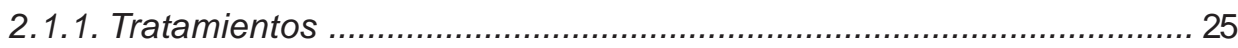

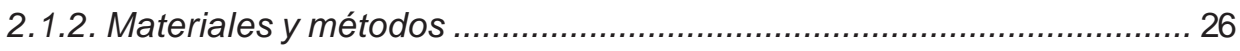

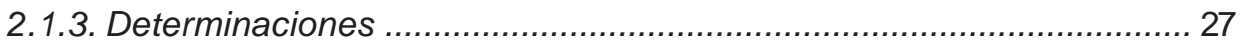

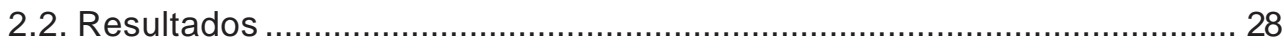

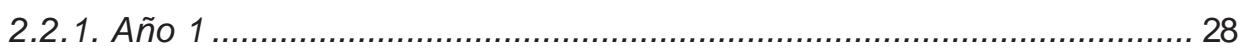

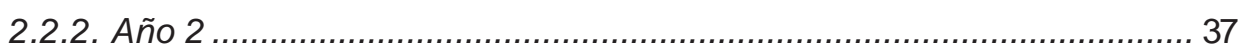

2.2.3. Comentarios y conclusiones de ambos años $(1+2)$ en conjunto ...... 45 


\section{Página}

3.EVALUACIÓN DE NOVILLOS SOBRE SORGO FORRAJERO CON DISTINTAS CARGAS Y TIPOS DE SUPLEMENTACIÓN

3.1. Evaluación del uso de diferentes suplementos sobre sorgo forrajero ......... 46

3.1.1. Materiales y métodos .................................................................. 46

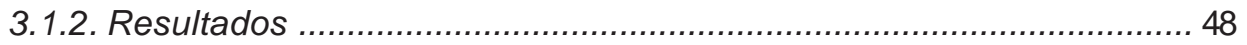

3.1.3. Comentarios y conclusiones de ambos años $(1+2)$ en conjunto ...... 62

3.2. Evaluación del uso de diferentes suplementos y cargas sobre sorgo

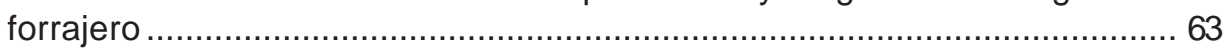

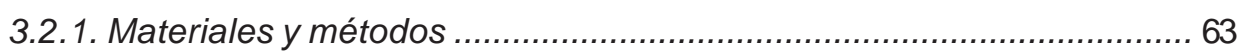

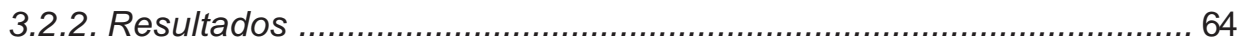

3.3. Evaluación del uso de diferentes suplementos sobre sorgo forrajero

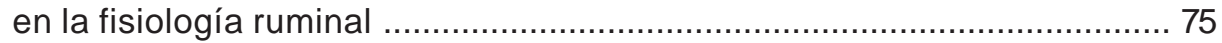

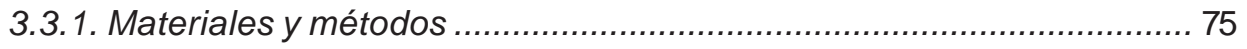

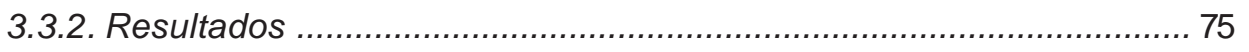

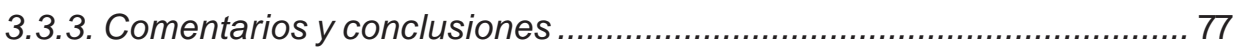

4. RESUMEN Y DISCUSIÓN CONJUNTA DE LA INFORMACIÓN PRESENTADA . 77

4.1. Base forrajera: sorgo híbrido vs. sudangrás .......................................... 77

4.2. Manejo del pastoreo y comportamiento animal ........................................ 77

4.3. Producción forrajera y producción animal ................................................ 79

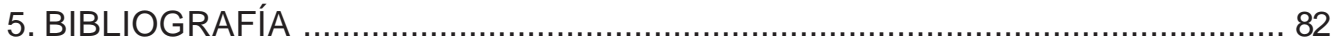

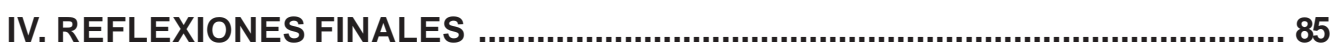

1. INTEGRACIÓN DE RESULTADOS DE ENSAYOS NACIONALES DE RECRÍA Y ENGORDE DE NOVILLOS SOBRE SORGOS FORRAJEROS 85

2. IMPLICANCIAS Y RECOMENDACIONES PRÁCTICAS DE ESTA LÍNEA DE

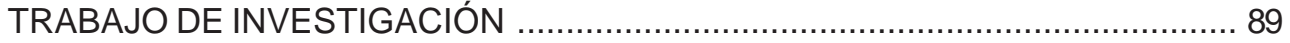

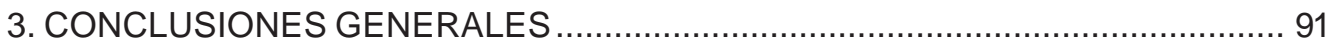

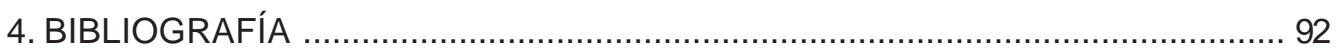

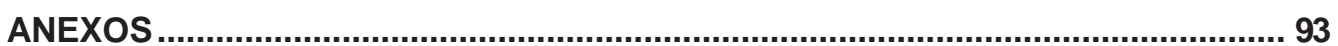




\section{PRÓLOGO}

En un contexto de intensificación sostenible de la producción bovina del Uruguay, la mejora de los procesos de recría y engorde conforman aspectos clave para aumentar la producción de carne por unidad de superficie y la ganancia individual, calidad de producto y su proyección positiva sobre el ingreso y la calidad de vida de los productores, lo cual ha sido demostrado por INIA y otras organizaciones nacionales de investigación y transferencia de tecnología, así como por productores individuales o grupos de productores que comparten su información productiva y económica (FUCREA, IPA, etc.).

Puntualmente durante los meses estivales, y en particular en la ganadería extensiva y semi-extensiva, las restricciones están dadas por un desbalance entre los requerimientos energéticos animales con la oferta de nutrientes, reflejado por una insuficiente calidad o cantidad de forraje. A ello se suma que las máximas variaciones en la producción forrajera se registran durante el verano, inestabilidad que puede ser atenuada con la implantación y posterior utilización de verdeos anuales estivales. La región del Basalto es un fiel reflejo de esta variabilidad en la producción y valor nutritivo de forraje que afecta la producción animal.

Si bien se encuentran afectados por la variabilidad interanual de las relaciones de precios de insumos y productos, así como por el efecto adicional de la variabilidad climática, generalmente en la ganadería los verdeos anuales estivales pueden ser una alternativa productiva y económica que puede dar estabilidad a la producción de forraje de los sistemas productivos ganaderos, otorgándoles una mayor resiliencia y sostenibilidad.

La lógica productiva y económica de la aplicación de esta alternativa tecnológica requiere de un conocimiento preciso de las relaciones de causalidad entre los diferentes elementos que hacen a su instrumentación y su respuesta animal y económica. Los verdeos de verano se caracterizan por producir altas cantidades de forraje en un tiempo acotado del período estivalotoñal, pero con deficiencias en cuanto al valor nutricional del forraje para impactar en forma sustantiva en la productividad individual de los animales, por lo cual su mayor ventaja en los sistemas ganaderos es su alta capacidad de carga. Pero en un contexto de aumento de la productividad y de relaciones insumo/producto desafiantes, es necesario explorar nuevas variantes en el uso de estos verdeos que permitan optimizar su uso a nivel de todos los sistemas productivos. En este sentido, existe una serie de limitantes en cuando a la disponibilidad de la información proveniente de la investigación nacional, especialmente en cuanto a su aplicación a nivel de sistemas ganaderos extensivos o semi-extensivos, como es el caso de la región de Basalto. Esta región ocupa una importante proporción de la ganadería nacional ha visto aumentado su rol como proveedora de animales terminados para abastecer la faena nacional.

Los trabajos experimentales de la presente publicación tuvieron por objetivo cuantificar la respuesta animal (por unidad de superficie y como producción individual) frente a la aplicación de variantes en el manejo de diferentes materiales genéticos de sorgo forrajero, la capacidad de carga y la suplementación con diferentes opciones de granos de cereales o sub-productos de la industria cerealera nacional. De esta forma se generaron coeficientes técnicos que permiten al productor y/o a su asesor proyectar y predecir performances individuales y producciones por hectárea, y por ende estimar diferentes escenarios de ingreso económico por la aplicación de las tecnologías que se proponen en esta publicación. Con estos resultados de la investigación de INIA, complementada con la proveniente de otros organismos de investigación nacional e internacional, las propuestas tecnológicas resumidas y sistematizadas en esta publicación deberán adaptarse a las condiciones particulares de cada año y de cada empresa para su potencial aplicación comercial, de acuerdo a los intereses y expectativas de los productores y su entorno. 


\section{AGRADECIMIENTOS}

El cuerpo técnico del INIA responsable de llevar adelante esta publicación, quiere agradecer muy especialmente a:

- Alos investigadores del INIA Tacuarembó, y en particular a Robin Cuadro, Diego Giorello, Gustavo Brito y América Mederos.

- $\quad$ Al personal de apoyo de INIA Tacuarembó: Sergio Daniel Bottero, Yovana Martínez, Julio Frugoni, Gonzalo Escayola, Juan Levratto Wilfredo Zamit, Mauro Bentancur, Julio Costales, Guillermo De Souza, Franco Rodríguez, Héctor Rodríguez, Eduardo Moreira, Fernando Icatt, Matías Barboza, Matías Martínez, Griselda Altez, Natalia Lemos.

- Al personal de administración, secretaría, difusión, operaciones, mantenimiento y recursos humanos, pero particularmente a la Lic. Carolina Pereira, Zenia Barrios y Manuel Viera.

— A Raúl Gómez-Miller y Laura Orrego por la revisión de esta edición. 


\section{INTRODUCCIÓN}

Lagomarsino, Ximena*

Cazzuli, Fiorella**

Montossi, Fabio***

\section{INTRODUCCIÓN}

\subsection{Contexto de la producción, faena y mercado de carne vacuna de la región de Basalto y del Uruguay}

Si bien a nivel mundial la producción ganadera uruguaya representa solamente el $0,77 \%$ en términos de cabezas de ganado, las exportaciones nacionales representan el $3,4 \%$ del mercado mundial de carne bovina (FAO, 2013).

En el año 2013, las exportaciones de carne bovina uruguaya correspondieron al $80 \%$ del total de lo producido a nivel nacional, siendo la composición de esta exportación la siguiente: 80,16 y 4 \% para carne congelada, carne enfriada y carne elaborada y/o salada, respectivamente (DIEA, 2014).

El valor de la tonelada de carne bovina exportada por Uruguay en el año 2014 promedió los $4.038 \mathrm{U} \$ \mathrm{~S} / \mathrm{t}$, siendo los principales destinos de colocación Asia (30\%), Unión Europea (29\%), países del NAFTA (17\%) y resto de mundo (24\%) (INAC, 2015).

Según un estudio llevado a cabo por Rava et al. (2014) sobre la producción de carne vacuna para exportación, esta es una actividad económica muy competitiva, constituyendo la base de las exportaciones del país (15\% del total). En este estudio, se concluye que en términos generales, esta cadena transfiere una muy alta cantidad de recursos hacia otros sectores de la economía nacional, a través de la transferencia directa (40\%) y de impuestos y cargas sociales (60\%).

En cuanto a la importancia de los novillos en la faena nacional, de las más de 2 millones de cabezas faenadas en el año 2014, esta categoría representó el $53 \%$ del total de faena (INAC, 2015). El promedio de peso vivo en pie en planta fue de $510,5 \mathrm{~kg}$ con un rendimiento $53,13 \%$, lo que resulta en un promedio de $271,2 \mathrm{~kg}$ de peso canal (caliente) en $4^{\text {ta }}$ balanza. Al discriminar la faena de los novillos, se observa que para este mismo año, el $6 \%$ correspondía a novillitos diente de leche, $40 \%$ a novillos $2-4$ dientes, $20 \%$ a novillos 6 dientes y el restante $34 \%$ a novillos boca llena. Asimismo, en cuanto a la distribución estacional de la faena de novillos, esta se encuentra distribuida en 24 , 23,24 y $30 \%$ para las estaciones de invierno, primavera, verano y otoño, respectivamente (INAC, 2014).

A nivel productivo, económico y social, la región de Basalto presenta importancia destacada para el país, extendiéndose desde Artigas hasta Río Negro $(23,22 \%$ de la superficie agrícola útil del país). La principal base forrajera son las pasturas naturales, mientras que el área mejorada ocupa una superficie del 4,8 al 5,8 \% del total. A pesar que las praderas y los campos mejorados siguen ocupando una mayor superficie en comparación al campo fertilizado y los verdeos anuales, la superficie de estos ha aumentado durante los últimos años. El $38 \%$ del rodeo nacional se encuentra en esta región (Berreta et al., 2014).

\footnotetext{
* Ing. Agr. Programa Nacional de Producción de Carne y Lana, INIA Tacuarembó (2011-2015)

** Ing. Agr. MSc. Programa Nacional de Producción de Carne y Lana, INIA Tacuarembó.

*** Ing. Agr. PhD. Director Nacional de INIA.
} 


\subsection{Contexto y justificación de la propuesta tecnológica desarrollada en esta publicación}

Durante los últimos 15 años, a nivel de los sistemas ganaderos del norte del país se registraron importantes cambios cualitativos y cuantitativos, entre los que se destaca un incremento del área agrícola y del área forestada. Estos incrementos, combinados con el hecho de que el mercado financiero demostraba interés en realizar inversiones seguras y rentables, determinaron un aumento del precio y la renta de la tierra. Esta nueva realidad en la región determinó un aumento del stock bovino y su perfil productivo, es decir, se intensificaron los procesos de recría e invernada (Montossi et al., 2014). Esta fue la manera que encontró la ganadería para mantenerse competitiva frente a otras alternativas productivas más rentables.

El desarrollo e implementación de estrategias de alimentación y manejo especializadas que potencien la producción y maximicen la eficiencia de la producción de carne bovina, determinan mejoras en la productividad y eficiencia de la ganadería, disminuyendo la edad de faena (Soares de Lima y Montossi, 2010).

El engorde estival de novillos de sobreaño ha sido identificado como una de las limitantes en la mejora de la productividad de los sistemas ganaderos en particular en las condiciones agroecológicas imperantes en la región del Basalto (Lagomarsino y Montossi, 2014). Por otra parte, la inclusión de un área reducida mejorada de uso intensivo permitiría darle sostenibilidad productiva y económica a los sistemas productivos basados en el campo natural. Esta es una alternativa para viabilizar esquemas productivos que tengan como objetivo mantener y/o conservar la estabilidad y productividad de los buenos campos naturales del Basalto (Montossi et al., 2014). En este contexto, entre otras opciones de inclusión de pasturas mejoradas en los esquemas forrajeros de los sistemas productivos, la introducción de verdeos estivales -para acelerar los procesos de recría e invernada- podrían ser una alternativa forrajera válida para incrementar la productividad e ingreso de estos sistemas de la región basáltica.

Es bien conocida la variación en cantidad y distribución del régimen pluviométrico de la región basáltica. Las precipitaciones medias anuales varían entre 1100 y 1300 $\mathrm{mm}$, aumentando desde el sur hacia el norte, existiendo una irregularidad de las mismas, tanto en periodicidad como en intensidad, manifestándose en sequías e inundaciones que pueden ocurrir en distintas estaciones del año (Berreta,1998).

La siembra de cultivos anuales como los verdeos estivales es una práctica que se realiza a fin de asegurar una producción elevada y estable de los establecimientos ganaderos (Sawchik, 2012). En este sentido, los sorgos forrajeros presentan buena tolerancia a períodos de déficits hídricos (Fassio et al., 2002) y por lo tanto son altamente valorados en este contexto. Los cultivos forrajeros anuales estivales y la suplementación con granos, subproductos de la agricultura y concentrados elaborados, se tornan especialmente útiles para levantar las limitantes que se presentan en el verano en Uruguay (Lagomarsino y Montossi, 2014) y en esta región en particular.

Dependiendo del esquema del sistema productivo implementado y la orientación productiva y comercial del mismo, el incrementar la productividad y su estabilidad estival puede ser una estrategia clave para acelerar el proceso de intensificación de la ganadería en general y en particular de las etapas finales del proceso de engorde de novillos. En este contexto, los verdeos anuales estivales adquieren una relevancia estratégica para cumplir con ambos objetivos. Por ello, una finalización de la etapa de invernada vacuna durante el verano permitiría extraer del sistema a una categoría adulta con altos requerimientos de consumo de forraje, antes de la llegada del invierno, e incrementar la productividad global del predio. 


\section{OBJETIVO}

El objetivo de esta publicación es presentar la información generada por INIA durante 4 años con relación al engorde de novillos sobre verdeos anuales estivales de sorgos forrajeros en pastoreo para las condiciones agroecológicas del Basalto durante el período estival.

Esta línea de trabajo experimental incluyó los siguientes factores de evaluación:

a) material forrajero de Sorghum sp. (sorgos forrajeros),

b) carga animal,

c) nivel y tipo de suplemento y

d) las potenciales interacciones entre los factores mencionados.

Las evaluaciones incluyen los efectos de estos factores sobre las características y producción del cultivo, la producción individual, comportamiento animal, calidad de canal in vivo y producción por unidad de superficie.

La información que se presenta es clave para brindar herramientas tecnológicas a productores y/o técnicos que faciliten el análisis productivo y económico de la conveniencia de cada una de las tecnologías propuestas a nivel de esa etapa productiva de final de recría y terminación de novillos, o potencialmente, su influencia en la eficiencia biológica y/o económica a nivel de todo el sistema productivo.

\section{BIBLIOGRAFÍA}

BERRETA, E. 1998. Principales características climáticas y edáficas de la región de basalto en Uruguay. En: Berretta, E.J. (ed.). Seminario de actualización en tecnologías para basalto, INIA Tacuarembó. Montevideo: INIA. p 3 - 10. (Serie Técnica; 102)

BERRETA, E.J.; MONTOSSI, F.; BRITO, G. 2014. Introducción. En: Berretta, E.J.; Montossi, F.; Brito, G. (eds.). Alternativas tecno- lógicas para los sistemas ganaderos de basalto. Montevideo: INIA. (Serie Técnica 217).

FAO. 2013. FAOSTAT. Consultado 15 may. 2015 de: http://faostat3.fao.org/home/E

FASSIO, A.; CAZZOLINO, D.; IBAÑEZ, V.; FERNÁNDEZ, E. 2002. Sorgo: destino forrajero, INIA La Estanzuela. Montevideo: INIA. 30 p. (Serie Técnica 127).

INAC. 2014. Informe estadístico año agrícola julio 2013-junio 2014. Montevideo: INAC. $83 \mathrm{p}$.

INAC. 2015. DIAE/INAC. Consultado 15 may. 2015 de: http://www.inac.gub.uy/inac/ diae/faena.html

LAGOMARSINO, X.; MONTOSSI, F. 2014. Engorde estival de novillos en pastoreo sobre sorgos forrajeros con suplementación proteica. Revista INIA, 39: 17-22.

MONTOSSI, F.; SOARES DE LIMA, J.; BRITO, G.; BERRETTA, E.J. 2014. Impacto en lo productivo y económico de las diferentes orientaciones productivas y tecnologías propuestas para la región del basalto. En: Berretta, E.J.; Montossi, F.; Brito, G. (eds.). Alternativas tecnológicas para los sistemas ganaderos de basalto. Montevideo: INIA. p. 557-568. (Serie Técnica; 217)

RAVA, C.; FERRARO, B.; LANFRANCO, B. 2012. Competitividad y transferencias en la cadena cárnica bovina en Uruguay. Montevideo: INIA. 42 p. (Serie Técnica; 198).

SAWCHIK, J. 2012. Necesidades de riego en cultivos y pasturas. En: Seminario Internacional (2, 2012, Salto, Uruguay). Riego en cultivos y pasturas. Montevideo, UY. INIA p. 57-68.

SOARES DE LIMA, J.; MONTOSSI, F. 2010. Años muy buenos, años muy malos: El rol de la suplementación en sistemas ganaderos extensivos en un contexto de alta variabilidad climática y de producción de forraje. Revista INIA, 22: 16 - 20.

URUGUAY. MGAP, DIEA. 2014. Anuario estadístico agropecuario 2014. Montevideo: DIEA. 243 p. 


\section{INTRODUCCIÓN}

El objetivo de este capítulo es recopilar la información experimental que se presenta más adelante en esta misma publicación, de manera de poder conocer los antecedentes de la investigación y realizar una comparación entre los resultados obtenidos previamente, con los de la información producida por esta línea de investigación.

En primera instancia, se describe brevemente la base forrajera sobre la que se realizaron los ensayos (sorgos), detallando características de los distintos tipos de sorgos forrajeros: sudangrás y sorgos forrajeros híbridos ("clásicos", con el gen BMR y fotosensitivos).

Luego, se mencionan algunas generalidades sobre la suplementación de novillos en pastoreo sobre verdeos estivales.

Finalmente, luego de presentar los requerimientos animales energéticos y proteicos de novillos en fase de terminación, se describen las experiencias extranjeras y nacionales con relación al engorde de novillos sobre verdeos de verano.

\section{SORGOS FORRAJEROS (SORGHUM SP.)}

Douglas (1980), citado por Silungwe (2011), clasifica a los sorgos forrajeros en tres grupos: sudangrás, híbridos de sorgo $\mathrm{x}$ sudangrás y sorgo dulce. Los tres presentan características tales que pueden ser cultivados como una pastura de alta producción de aceptable calidad (Watson et al., 1993, citados por Silungwe, 2011).
En Uruguay, el desarrollo de especies templadas en los meses del verano no es el óptimo debido a las condiciones que se presentan en términos de humedad y temperatura. Los verdeos de verano de especies subtropicales, como el sudangrás, sorgo y sus híbridos, son ideales para llenar estos momentos de crisis forrajera (Vaz Martins et al., 2003).

El uso de distintas variedades de sudangrás y sorgo forrajero para pastoreo directo de los animales se ha generalizado en regiones ganaderas del Uruguay. El pastoreo de verdeos de verano permite mejorar la ganancia de peso de bovinos y aumentar la capacidad de carga y productiva del predio (Rovira y Echeverría, 2013).

Estos materiales se caracterizan por tener una elevada producción total de forraje por unidad de superficie (8000 a 15000 kilos de materia seca por hectárea - kg MS/ha) que permite sostener una alta carga animal durante los meses de verano (4 a 6 animales/ha) (Rovira y Echeverría, 2013).

En cuanto a su valor nutritivo, ContrerasGovea et al. (2010) citando a Cummins (1981), resumen algunas características del cultivo en diferentes estadios de maduración, en el Cuadro 1.

Los sorgos son especies de metabolismo C4, lo cual los hace más eficientes que las plantas con metabolismo C3 (Fassio et al., 2002). Se caracterizan por presentar excelente macollaje, por lo tanto están bien adaptadas al pastoreo, además de contar con una elevada tolerancia a períodos de déficits hídricos. Aportan altos volúmenes de forraje de aceptable calidad si son maneja-

\footnotetext{
* Ing. Agr. MSc. Programa Nacional de Producción de Carne y Lana, INIA Tacuarembó.

** Ing. Agr. Programa Nacional de Producción de Carne y Lana, INIA Tacuarembó (2011-2015)

*** Ing. Agr. PhD. Director Nacional de INIA.
} 
Cuadro 1. Composición del sorgo forrajero y su valor nutritivo en diferentes estadios de madurez (Cummins, 1981, citado por Contreras-Govea et al., 2010).

\begin{tabular}{|ccccccccc|}
\hline Estadio fenológico & \multicolumn{2}{c}{ Componente (\%) } & & \multicolumn{2}{c|}{ DMO (\%) } & & \multicolumn{2}{c|}{ FDA (\%) } \\
\cline { 2 - 3 } & Hoja & Tallo & & Hoja & Tallo & & Hoja & Tallo \\
\hline Lechoso tardío & 24 & 57 & 38 & 55 & & 35 & 39 \\
Pastoso temprano & 20 & 53 & & 44 & 55 & & 35 & 39 \\
Pastoso & 18 & 47 & & 46 & 53 & & 38 & 3 \\
Pastoso duro & 16 & 45 & & 44 & 54 & & 40 & 38 \\
\hline
\end{tabular}

Nota: $\mathrm{DMO}$ = digestibilidad de la materia orgánica in vitro; FDA = fibra detergente ácido.

dos correctamente en pastoreo. Una posible limitante que pueden presentar los sorgos forrajeros es su bajo contenido de proteína, la cual no es suficiente para mantener la producción de animales de alto potencial de crecimiento (Carámbula, 2007).

Cuanto más drásticas son las defoliaciones y se realizan más frecuentemente, decrece de forma notable la producción de forraje total (Carámbula, 1964). Lagomarsino y Montossi (2014) sostienen que el buen aprovechamiento de los sorgos forrajeros depende fundamentalmente del manejo del pastoreo aplicado y la carga animal utilizada. Este concepto es reforzado por Vaz Martins et al. (2003) quienes sostienen que podría ser más importante realizar un correcto manejo del material seleccionado, antes que la propia elección del tipo de sorgo a utilizar.

Gallarino (2008) menciona que uno de los problemas que se presentan a la hora de manejar los sorgos forrajeros es su crecimiento inicial -especialmente el tipo sudanya que este es muy rápido y como consecuencia de ello, los sorgos maduran rápidamente perdiendo su calidad y disminuyendo su producción futura.

Los sorgos forrajeros tienen el potencial de liberar ácido cianhídrico (HCN) como un mecanismo de adaptación contra los herbívoros (Wheeler et al., 1990; Busk y Moller, 2002; citados por Silungwe, 2011). Si la concentración de ácido cianhídrico es lo suficientemente elevada, puede ocurrir el envenenamiento del ganado en pastoreo (Fjell et al., 1991, citados por Silungwe, 2011).

La concentración de HCN que se considera segura para los animales varía según diferentes fuentes. Por ejemplo, Fjell et al. (1991), citados por Silungwe (2011) reportaron que rangos de $0-600 \mathrm{mg} / \mathrm{kg}$ de $\mathrm{PV}$ de $\mathrm{HCN}$ son considerados seguros, mientras que concentraciones de 600-1000 mg/kg de $\mathrm{PV}$ son considerados potencialmente tóxicos. En contraste, Vogel et al. (1987), citados por Silungwe (2011) sostienen que concentraciones de $0-500 \mathrm{mg} / \mathrm{kg}$ de PV son poco probables que resulten en intoxicación de ganado, mientras que rangos de $500-750 \mathrm{mg} / \mathrm{kg}$ PV son considerados muy tóxicos. Por otro lado, Takamitsu (1973), citado por Silungwe (2011), especificó que 2,042 $\mathrm{mg} / \mathrm{kg}$ PV de HCN puede envenenar al animal que consuma un sorgo con estas características y provocar su muerte.

\subsection{Sudangrás (Sorghum sudanense)}

Dentro del grupo de los sorgos, el sudangrás se destaca netamente del resto por sus características morfofisiológicas y sus atributos agronómicos. Es una especie con plantas muy macolladoras, con tallos delgados, sólidos, que rara vez superan los $6 \mathrm{~mm}$ de diámetro y que poseen gran cantidad de hojas. Resiste muy bien al pisoteo y tiene un excelente rebrote, el cual se hace mayor a medida que avanza su ciclo. Esta especie produce en promedio un total de $9000 \mathrm{~kg} \mathrm{MS} / \mathrm{ha}$. La digestibilidad de la materia orgánica (DMO) oscila entre 53,7 y $69,8 \%$ y el contenido de proteína cruda (PC) oscila entre un máximo de 14,1 y un mínimo de 7,7 \% (Carámbula, 2007).

No obstante, el sudangrás tiene un rendimiento menor en comparación, por ejemplo, con los sorgos híbridos (Silungwe, 2011). 


\subsection{Sorgos híbridos (Sorghum x S. sudanense)}

Para poder superar las ventajas productivas del sudangrás, los mejoradores genéticos cruzaron sudangrás por sorgo para producir variedades con mejores valores nutritivos y de productividad que el sudangrás (Kalton, 1988; Miron et al., 2007; Moyer et al., 2004; citados por Silungwe, 2011). Así, fueron generados los materiales híbridos, resultantes de la cruza de Sorghum bicolor x Sorghum sudanense (Silungwe, 2011).

Los sorgos híbridos presentan tallos más gruesos y hojas más anchas que los de sudangrás y se caracterizan por poseer una mayor precocidad, lo que les permite ser pastoreados antes. Los rendimientos de forraje totales son mayores que los de sudangrás (Carámbula, 2007). Su modalidad de crecimiento y sus rendimientos elevados en un período corto de tiempo crean problemas en su utilización y en el mantenimiento de su valor nutritivo (Hoveland, 1968, citado por Carámbula, 2007).

Si bien el potencial productivo del sorgo hibrido es elevado, este puede ser limitado en mayor proporción que en el sudangrás cuando se efectúan cortes más frecuentes (Carámbula, 2007).

Giorello et al. (2012) reportaron producciones totales de sorgo híbrido en régimen de secano de $10.066 \mathrm{~kg} \mathrm{MS} / \mathrm{ha}$. Por otro lado, Bianco et al. (2003) reportaron disponibilidades de forraje de 3806, 842 y $1185 \mathrm{~kg}$ MS/ha para un sorgo híbrido para el primer, segundo y tercer pastoreo, respectivamente. Estos últimos autores reportaron contenidos de proteína cruda (PC) de 15,1 y 12,7 \% para el segundo y tercer ciclo de pastoreo, respectivamente.

Los sorgos del tipo nervadura marrón o Brown Middle Rib (BMR) tienen la particularidad que cuentan con una digestibilidad mayor que el Sorghum bicolor, dado su menor lignificación (Vasconcelos et al., 2003; Casler et al., 2003; Mc Cuistion et al., 2005; Schwarz et al., 2008; Contreras-Govea et al., 2010).

Los sorgos BMR cuentan con mayor calidad como forraje fresco que se traduce en una mayor producción de carne, siempre que esta opción forrajera esté acompañada de un

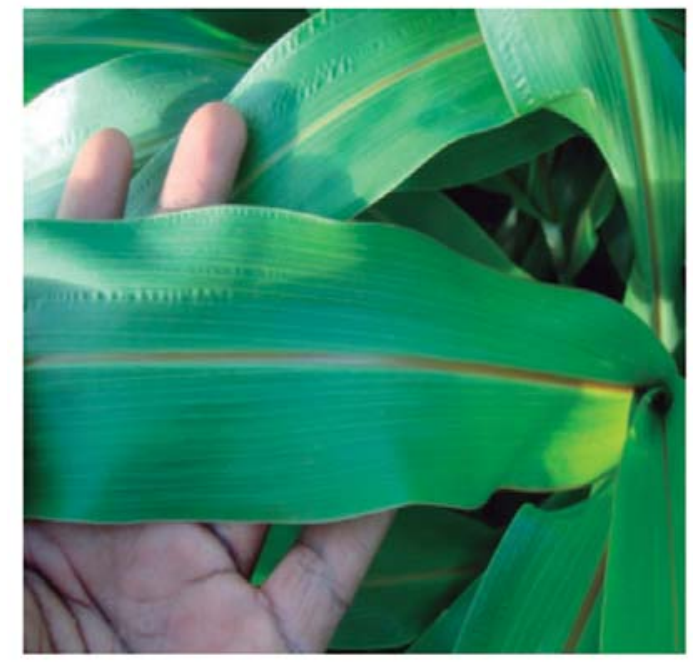

Figura 1. Sorgo de nervadura marrón o BMR (Silungwe, 2011).

aporte adicional de proteína, un correcto manejo (intensidad de pastoreo) y una adecuada asignación de forraje (Fernández Mayer et al., 2011ab).

A pesar que el gen BMR aporta características deseables en forrajes de verano, generalmente se asocia con rendimientos en materia seca menores y con un menor vigor de las plantas (Casler et al., 2003; Miller y Stroup, 2003; Pedersen et al., 2005; citados por Silungwe, 2011). A partir de esta situación, los mejoradores genéticos trabajaron en la creación de nuevos híbridos con mayor vigor y alto rendimiento productivo (Miller y Stroup, 2003, citados por Silungwe, 2011).

Bianco et al. (2003) reportaron disponibilidades de forraje de sorgo BMR de 3901, 772 y 1148 kg MS/ha, para el primer, segundo y tercer ciclo de pastoreo, respectivamente. Estos autores reportaron 13,3 y 12,0 \% de PC para el segundo y tercer ciclo de pastoreo, respectivamente.

Por otra parte, actualmente se cuenta con sorgos del tipo "fotosensitivos". Según Mc Cuistion et al. (2005), las variedades de sorgos fotosensitivos retrasan el inicio del período reproductivo de la planta hasta que la longitud del día no sea mayor que 12 horas con 30 minutos. La característica fotosensitiva de este tipo de materiales mejora la producción potencial de materia seca, al extender el período vegetativo de la planta (Mc Cuistion et al., 2011). 
Asimismo, Vasconcelos et al. (2003) plantean que los sorgos híbridos foto sensitivos, por lo general, presentan una mayor relación hoja/tallo, lo que se podría asociar con mayores digestibilidades del forraje.

\section{SUPLEMENTACIÓN DE BOVINOS SOBRE VERDEOS DE VERANO}

La suplementación en pastoreo es una de las principales herramientas para la intensificación de los sistemas ganaderos. Permite corregir dietas desbalanceadas, aumentar la eficiencia de conversión de las pasturas, mejorar las ganancias de peso de los animales y acortar los ciclos de engorde de los bovinos (Peruchena, 1999).

La suplementación también es una herramienta para aumentar la capacidad de carga de los sistemas productivos, incrementando la eficiencia de utilización de las pasturas en sus picos de producción y aumentando el nivel de producción por unidad de superficie (Leng, 1983, citado por Peruchena, 1999).

Según Pordomingo (2003), la respuesta en la mejora de la eficiencia total de producción se manifiesta a niveles de suplementación mayores al 0,75\% del PV. El mismo autor sostiene que el alimento combinado de forraje y suplementos debe ser de alta digestibilidad para que se exprese una mejor conversión de alimento en aumento de peso. A niveles de suplementación por debajo del $0,5 \%$ del PV el efecto de sustitución es poco importante y podría no afectar el consumo de forraje.

Peruchena (1998), citado por Peruchena (1999) y Peruchena (1997), citado por Peruchena, (2003) estudiaron la suplementación de novillos en la fase de terminación durante el verano, si bien para este caso fue sobre una pastura perenne de Bracchiaria brizantha. Los animales lograron ganancias de $1,140,1,070$ y $0,510 \mathrm{~kg} / \mathrm{an} /$ día al ser suplementados con expeller de algodón al 0,6 \% del PV (suplementación proteica), suplemento balanceado energético-proteico al $1 \%$ del PV y testigo sin suplementación, respectivamente. La suplementación permitió terminar todos los novillos para faena. El investigador destaca la alta eficiencia de conver- sión de ración en peso vivo, reduciendo el aporte de las pasturas. En el caso de la suplementación proteica, la eficiencia fue de $3: 1$, mientras que con la suplementación energético-proteica la misma fue de 5,8:1.

\subsection{Tipos de suplementos}

Según de Ruiter et al. (2007), los suplementos son utilizados en la producción animal cuando los nutrientes provenientes del forraje no son suficientes para cubrir los requerimientos de los animales. Estos autores clasifican a los concentrados en energéticos o proteicos, según el principal aporte de un nutriente u otro.

Field (2007) considera energéticos a los suplementos que tienen menos de $20 \%$ de proteína y menos de $18 \%$ de fibra, mientras que los suplementos proteicos son definidos como alimentos con más de $20 \%$ de proteína.

En la práctica, se destacan en nuestras condiciones suplementos energéticos (grano de maíz), energético proteicos (afrechillo de arroz) y proteicos (expeller de soja y girasol).

El grano de maíz es un suplemento energético. Mc Donald et al. (2011) consideran a este alimento como una excelente fuente de energía digestible, si bien cuenta con muy baja concentración proteica de muy baja calidad. Estos autores reportan concentraciones de proteína cruda del orden de los 90-140 g/kg MS. Mieres (2004) reporta contenidos promedio de proteína cruda de 9,18 \% y energía metabolizable de 3,26 MCal/kg MS.

En cuanto al afrechillo de arroz, es un subproducto de la industria molinera arrocera y su uso es muy común en el país, especialmente en las zonas cercanas a estas industrias. Pittaluga et al. (2005) demostraron que este suplemento energético-proteico es una alternativa económica y biológicamente válida para mejorar el proceso de recría vacuna invernal, lo cual fue ratificado por Lagomarsino et al. (2014). Mc Donald et al. (2011) reportan en afrechillo de arroz contenidos de proteína cruda de 12-14,2 \% PC. Mieres (2004) reporta una concentración de 
Energía Metabolizable (EM) de $3 \mathrm{MCal} / \mathrm{kg}$ MS, 15,2 \% PC, 13,8 \% FDA (fibra detergente ácido), 31,7 \% FDN (fibra detergente neutro), 10,5\% Cen (cenizas) y $15 \%$ EE (extracto etéreo).

Los alimentos tipo "expeller" son ricos en proteína, conteniendo entre 20 y $50 \%$ de PC. Estos alimentos, en general, presentan alrededor del $95 \%$ del contenido de nitrógeno como proteína verdadera de excelente calidad biológica (Mc Donald et al., 2011).

Mieres (2004) reporta para expeller de soja, contenidos de $45,7 \%$ de PC y EM del orden de 3,15 MCal/kg MS.

Mc Donald et al. (2011) reportan valores de EM para el expeller de girasol del orden de 3,1 $\mathrm{MCal} / \mathrm{kg} \mathrm{MS}$. Estos autores sostienen que si bien este tipo de suplementos son fuentes del alto nivel de proteína en la dieta para los animales, puede ser limitante en contenido de lisina. Mieres (2004) reporta valores de 36,3\%

PC para este tipo de alimento.

\section{PERFORMANCE ANIMAL DE NOVILLOS SOBRE VERDEOS DE VERANO}

\subsection{Requerimientos de energía y proteína}

Se presentan los requerimientos de energía para mantenimiento y para engorde de vacunos adultos, según NRC (1996) en el Cuadro 2.

Por otro lado, se presentan los requerimientos de proteína metabolizable para mantenimiento y para engorde de vacunos adultos en el Cuadro 3.

Los animales con los que se trabajó en los experimentos para obtener los resulta-

Cuadro 2. Requerimientos de energía metabolizable para mantenimiento y engorde (MJ ME/día) para ganado en crecimiento y terminación (adaptado de NRC, 1996).

\begin{tabular}{|cccccc|}
\hline \multirow{2}{*}{$\begin{array}{c}\text { GMD } \\
\text { (kg/an/día) }\end{array}$} & $\mathbf{2 5 0}$ & $\mathbf{3 0 0}$ & $\mathbf{3 5 0}$ & $\mathbf{4 0 0}$ & $\mathbf{4 5 0}$ \\
\hline \multicolumn{5}{c}{ Energía metabolizable } & para mantenimiento \\
0 & 20,3 & 23,2 & 26,1 & 28,8 & 31,5 \\
\hline \multicolumn{5}{c}{ Energía } & metabolizable para ganancia de peso \\
0,500 & 6,3 & 7,2 & 8,1 & 9,0 & 9,8 \\
1,000 & 13,4 & 15,4 & 17,3 & 19,1 & 20,9 \\
1,500 & 21,0 & 24,0 & 27,0 & 30,0 & 32,6 \\
2,000 & 28,8 & 33,0 & 37,0 & 40,9 & 44,7 \\
\hline
\end{tabular}

Nota: GMD = ganancia media diaria; kg/an/día = kilos por animal por día; PV = peso vivo.

Cuadro 3. Requerimientos de proteína metabolizable para mantenimiento y engorde ( $\mathrm{g} / \mathrm{d}$ ía) para ganado en crecimiento y terminación (adaptado de NRC, 1996).

\begin{tabular}{|c|c|c|c|c|c|}
\hline \multirow{2}{*}{$\begin{array}{c}\text { GMD } \\
\text { (kg/an/día) }\end{array}$} & \multicolumn{5}{|c|}{ PV (kg) } \\
\hline & 250 & 300 & 350 & 400 & 450 \\
\hline \multicolumn{6}{|c|}{ Proteína metabolizable necesaria para mantenimiento } \\
\hline- & 239 & 274 & 307 & 340 & 371 \\
\hline \multicolumn{6}{|c|}{ Proteína metabolizable necesaria para crecimiento } \\
\hline 0,500 & 155 & 158 & 157 & 145 & 133 \\
\hline 1,000 & 300 & 303 & 298 & 272 & 246 \\
\hline 1,500 & 440 & 442 & 432 & 391 & 352 \\
\hline 2,000 & 577 & 577 & 561 & 505 & 451 \\
\hline
\end{tabular}

Nota: GMD = ganancia media diaria; kg/an/día = kilos por animal por día; PV = peso vivo. 
dos del próximo capítulo, en términos generales, se encuentran entre pesos superiores a $300 \mathrm{~kg}$ PV y menores a $400 \mathrm{~kg}$ PV.

\subsection{Antecedentes experimentales}

\subsubsection{Experiencias extranjeras}

\subsubsection{Sin suplementación}

Berti (2011) realizó un ensayo en el Chaco Argentino (ambiente subtropical), en la que se evaluó la suplementación azufrada sobre la ganancia de novillos a pastoreo directo sobre sudangrás ad libitum. Los animales eran cruza cebú con un PV inicial de $280 \mathrm{~kg}$ PV y con 16 meses de edad. Los tratamientos fueron: testigo $(\mathrm{T})$; suplementación mineral que incluía azufre (S); suplementación mineral sin azufre (MIN). El Cuadro 4 resume algunos de los resultados de este ensayo.

Se reportaron valores de ácido cianhídrico (HCN) en planta promedio de 40,7 ppm, no afectando la performance animal. La digestibilidad de la materia orgánica (DMO) pro- medio fue de $45,8 \%$. Si bien no se registraron diferencias significativas entre las ganancias obtenidas $(P>0,05)$ en todo el período, durante los primeros 39 días de ensayo se constataron menores ganancias para los dos tratamientos suplementados, efecto que se revirtió luego del rebrote del verdeo.

Vasconcelos et al. (2003) llevaron a cabo un ensayo en Texas, Estados Unidos con novillos pastoreando dos materiales de sorgos híbridos tipo BMR (BMR I y II) y fotosensitivos (FS I y II). Se utilizaron novillos cruza con un peso inicial promedio de $250 \mathrm{~kg}$ PV. Se presentan algunos resultados de esta experiencia en el Cuadro 5.

Unos años más tarde, el mismo equipo de investigación de Texas (Mc Cuistion et al., 2005) llevó a cabo un ensayo con novillos cruza pastoreando distintos tipos de sorgos forrajeros, comparando: un material fotosensitivo (FS), dos materiales BMR exclusivamente y uno fotosensitivo con BMR (ambas características combinadas en el mismo material; FS+BMR). Los novillos iniciaron el ensayo con $213 \mathrm{~kg}$ PV y la pastura

Cuadro 4. Efecto del tipo de suplementación sobre performance de novillos pastoreando sudangrás (adaptado de Berti, 2011).

\begin{tabular}{|l|c|c|c|}
\hline Parámetros medidos & T & S & MIN \\
\hline Disponibilidad inicial promedio (kg MS/ha) & \multicolumn{3}{|c|}{8531} \\
Disponibilidad final promedio (kg MS/ha) & 5514 \\
Relación hoja/tallo inicial & \multicolumn{3}{|r|}{0,391} \\
Relación hoja/tallo final & 0,696 & 0,676 & 0,714 \\
\hline GMD (kg/an/día) &
\end{tabular}

Nota: $\mathrm{kg} \mathrm{MS/ha} \mathrm{=} \mathrm{kilos} \mathrm{de} \mathrm{materia} \mathrm{seca} \mathrm{por} \mathrm{hectárea;} \mathrm{GMD} \mathrm{=} \mathrm{ganancia} \mathrm{media} \mathrm{diaria;}$ $\mathrm{kg} / \mathrm{an} / \mathrm{día}=$ kilos por animal por día.

Cuadro 5. Efecto del pastoreo de distintos tipos de sorgo sobre novillos en crecimiento (adaptado de Vasconcelos et al., 2003).

\begin{tabular}{|lcccc|}
\hline Parámetro medido & FS I & FS II & BMR I & BMR II \\
\hline Disponibilidad inicial (kg MS/ha) & $2160^{\mathrm{b}}$ & $2496^{\mathrm{ab}}$ & $3000^{\mathrm{a}}$ & $2205^{\mathrm{b}}$ \\
Disponibilidad final (kg MS/ha) & 1763 & $1761^{\mathrm{a}}$ & 1185 & 867 \\
Hojas (\%) & $59^{\mathrm{b}}$ & $67^{\mathrm{b}}$ & 61 & 65 \\
GMD (kg/an/día) & $1,020^{\mathrm{b}}$ & $1,048^{\mathrm{b}}$ & $1,161^{\mathrm{b}}$ & $1,379^{\mathrm{a}}$ \\
Producción PV (kg/ha) & $457^{\mathrm{ab}}$ & $402^{\mathrm{b}}$ & $435^{\mathrm{ab}}$ & $514^{\mathrm{a}}$ \\
\hline
\end{tabular}

Nota: $\mathrm{kg} \mathrm{MS} / \mathrm{ha}=$ kilos de materia seca por hectárea; GMD = ganancia media diaria; kg/an/día = kilos por animal por día; $\mathrm{PV}=$ peso vivo; Valores de una misma línea evaluado con letras distintas son significativamente distintas $(P<0,10)$. 
Cuadro 6. Efecto del pastoreo de distintos tipos de sorgo sobre novillos en crecimiento (adaptado de Mc Cuistion et al., 2005).

\begin{tabular}{|lcccc|}
\hline Parámetro medido & FS & BMR 1 & BMR 2 & FS+BMR \\
\hline Disponibilidad inicial (kg MS/ha) & 2267 & 2052 & 2168 & 2163 \\
Hojas (\%) & $59^{\mathrm{a}}$ & $58^{\mathrm{ab}}$ & $55^{\mathrm{b}}$ & $55^{\mathrm{b}}$ \\
GMD (kg/an/día) & $0,903^{\mathrm{c}}$ & $1,157^{\mathrm{ab}}$ & $1,202^{\mathrm{a}}$ & $1,034^{\mathrm{b}}$ \\
Producción PV (kg/ha) & $138^{\mathrm{b}}$ & 148 & $166^{\circ}$ & 145 \\
\hline
\end{tabular}

Nota: kg MS/ha = kilos de materia seca por hectárea; GMD = ganancia media diaria; kg/an/día = kilos por animal por día; PV = peso vivo; Valores de una misma línea evaluado con letras distintas son significativamente distintas $(P<0,01)$.

alcanzaba una altura de $60-76 \mathrm{~cm}$. El Cuadro 6 presenta algunos de los resultados obtenidos en este ensayo.

Los tres materiales conteniendo el gen BMR alcanzaron performances significativamente superiores $(P<0,01)$ al material FS. Sin embargo, no se encontraron diferencias significativas a nivel de la producción total de $P V / h a$.

Mc Cuistion et al. (2007) condujeron un ensayo contrastando dos tipos de sorgos híbridos: BMR versus fotosensitivo (FS) ofrecidos en pastoreo a novillos con un PV inicial de $238 \mathrm{~kg}$. El ensayo se repitió por tres años consecutivos, con un período promedio de 85 días, utilizando cargas de entre 3,2 y 6,8 cabezas/ha. Los animales ingresaban a pastorear con aproximadamente $60 \mathrm{~cm}$ de altura del forraje. En el Cuadro 7 se presentan algunos de los resultados logrados en este ensayo.

Los novillos pastoreando sobre sorgo FS lograron ganancias máximas a una carga de 5,0 cabezas/ha, mientras que las ganancias mínimas se registraron con una dotación de 5,9 cabezas/ha. Para el caso del BMR, la ganancia máxima obtenida se logró con una carga de 4,6 cabezas/ha, luego de lo cual, las ganancias disminuían hasta un mínimo registrado con la carga de 5,9 cabezas/ha. Por otra parte, la producción máxima de carne para el FS se alcanzó con 5,7 cabezas/ha, mientras que para el BMR la máxima producción se asoció a la dotación de 5,3 cabezas/ha.

Al haber sido realizado este ensayo por tres años, los investigadores pudieron obser-
Cuadro 7. Efecto del pastoreo de distintos tipos de sorgo sobre novillos en crecimiento (adaptado de Mc Cuistion et al., 2007).

\begin{tabular}{|lcc|}
\hline Parámetro medido & FS & BMR \\
\hline $\begin{array}{l}\text { Disponibilidad inicial } \\
\text { (kg MS/ha) }\end{array}$ & 1783 & 2264 \\
PC (\%) & $22^{\text {a }}$ & $20^{\text {b }}$ \\
GMD max (kg/an/día) & 0,962 & 1,100 \\
GMD min (kg/an/día) & 0,744 & 0,640 \\
Producción PV máx & 365 & 410 \\
(kg/ha) & & \\
\hline
\end{tabular}

Nota: $\mathrm{kg} \mathrm{MS/ha} \mathrm{=} \mathrm{kilos} \mathrm{de} \mathrm{materia} \mathrm{seca} \mathrm{por} \mathrm{hectá-}$ rea; GMD = ganancia media diaria; $\mathrm{kg} / \mathrm{an} / \mathrm{día}=$ kilos por animal por día; PV = peso vivo; Valores de una misma línea evaluado con letras distintas son significativamente distintas $(\mathrm{P}<0,05)$ (solo para el caso de disponibilidad y contenido de proteína cruda).

var variaciones debidas a condiciones ambientales entre otras fuentes de variación. Se concluye que a medida que la carga animal aumenta, la exactitud de la predictibilidad de la performance animal se reduce.

Mc Cuistion et al. (2011) continuaron la línea de trabajo recién presentada, trabajando con novillos cruza de razas Británica $x$ Continental, comparando sorgos FS contra BMR. El Cuadro 8 presenta algunos resultados del ensayo.

La producción de peso vivo por hectárea alcanzó un máximo para BMR con cargas más elevadas que en el caso del sorgo FS y declinó más pronunciadamente a medida que la carga animal se incrementaba.

Debido a la selectividad animal en pastoreo, el estado de madurez de las plantas y 
Cuadro 8. Efecto del pastoreo de distintos tipos de sorgo sobre novillos en crecimiento (adaptado de Mc Cuistion et al., 2011).

\begin{tabular}{|lcc|}
\hline Parámetro medido & FS & BMR \\
\hline \hline $\begin{array}{l}\text { Disponibilidad inicial } \\
\text { (kg MS/ha) }\end{array}$ & 2777 & 3130 \\
PC (\%) & 21 & 19 \\
GMD máx (kg/an/día) & 0,900 & 0,930 \\
$\begin{array}{l}\text { Producción PV máx } \\
\text { (kg/ha) }\end{array}$ & 394 & 420 \\
\hline
\end{tabular}

Nota: $\mathrm{kg} \mathrm{MS/ha} \mathrm{=} \mathrm{kilos} \mathrm{de} \mathrm{materia} \mathrm{seca} \mathrm{por} \mathrm{hectárea;}$ $\mathrm{PC}=$ proteína cruda; $\mathrm{GMD}$ = ganancia media diaria; $\mathrm{kg} / \mathrm{an} /$ día = kilos por animal por día; $\mathrm{PV}=$ peso vivo; Valores de una misma línea evaluado con letras distintas son significativamente distintas $(p<0,05)$ (solo para el caso de disponibilidad y contenido de proteína cruda).

senescencia de las hojas, se registró una restricción de la disponibilidad de nutrientes para los animales al final del período de crecimiento de los cultivos, lo que limitó las ganancias de peso por deficiencias en el contenido de energía y/o proteína de la dieta. De esto se deduce que podría ser en este punto de la estación de pastoreo en que un programa de suplementación que provea una fuente adicional de proteína y/o energía pueda ser de utilidad para mitigar la restricción de nutrientes.

En Río Grande do Sul, Brasil, Coser y Maraschin (1983) realizaron un ensayo sobre sorgo forrajero para evaluar el desempeño con animales cruza cebú y cruza Charolais (edad $12-15$ meses y PV inicial $=190 \mathrm{~kg}$ ). El ensayo tuvo una duración total de 140 días (desde 21 de diciembre hasta 9 de mayo). Las disponibilidades de forraje inicial, media y final fueron de 4100, 2600 y 1200 kg MS/ha, respectivamente, mientras que la dotación animal inicial, media y final fue de $18,4,5,2$ y 4,0 animales/ha, respectivamente. En cuanto a la producción animal, la ganancia inicial, media y final fue de 0,100, 0,110 y 0,710 kg/an/día, respectivamente, si bien se registró un máximo de 1,340 kg/an/día en el tercer ciclo de pastoreo. En promedio, se obtuvieron ganan-

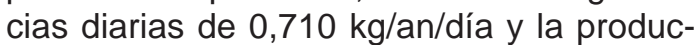
ción de carne se estimó en 401 kg PV/ha para todo el período experimental.

También en Rio Grande do Sul, Restle et al. (2002) evaluaron la performance de noviIlos cruza Charolais $x$ Nelore en fase recría $(\mathrm{PV}$ inicial $=211 \mathrm{~kg}$; edad promedio $14 \mathrm{me}$ ses) sobre sorgo forrajero (Sorghum bicolor), desde los primeros días de enero hasta mediados de abril (98 días en total). La masa de forraje objetivo al ingreso del pastoreo era de $2000 \mathrm{~kg} \mathrm{MS} / \mathrm{ha}$. Los animales finalizaron el período de evaluación con $330 \mathrm{~kg} \mathrm{PV}$. Algunos resultados de esta experiencia se presentan en el Cuadro 9.

Aita (1995), citado por Ribas y Machado (2009), reportó en Río Grande do Sul los resultados que se presentan en el Cuadro 10 en un ensayo con novillos de razas carniceras, pastoreando sobre un sorgo híbrido.

Los resultados presentados corresponden al promedio de un período experimental desde el $1^{\circ}$ de enero hasta el 24 de abril. Se destaca que durante el cuarto ciclo de pastoreo (desde 27 de marzo hasta 23 de abril),

Cuadro 9. Evaluación de desempeño de novillos pastoreando sobre sorgo forrajero (adaptado de Restle et al., 2002).

\begin{tabular}{|lccccc|}
\hline Parámetro medido & Ciclo 1 & Ciclo 2 & Ciclo 3 & Ciclo 4 & Media-Total \\
\hline Disponibilidad (kg MS/ha) & 1408 & 2260 & 2230 & 2258 & 2039 \\
PC (\%) & 12,8 & 10,1 & 8,5 & 8,5 & 9,95 \\
DMO (\%) & 67,5 & 55,9 & 48,6 & 46,3 & 54,6 \\
Carga total (kg PV/ha) & 1272 & 1416 & 1496 & 1353 & 1389 \\
GMD (kg/an/día) & 1,295 & 1,330 & 1,112 & 0,370 & 1,121 \\
Producción PV (kg/ha) & 190 & 199 & 159 & 22 & 570 \\
\hline
\end{tabular}

Nota: kg MS/ha = kilos de materia seca por hectárea; $\mathrm{PC}=$ proteína cruda; $\mathrm{DMO}$ = digestibilidad de la materia orgánica; $\mathrm{GMD}$ = ganancia media diaria; kg/an/día = kilos por animal por día; $\mathrm{PV}$ = peso vivo . 
Cuadro 10. Evaluación de desempeño de novillos pastoreando sobre sorgo forrajero híbrido (adaptado de Aita, 1995, citado por Ribas y Machado, 2009).

\begin{tabular}{|lc|}
\hline Parámetro medido & Promedio \\
\hline PC (\%) & 10 \\
DMO (\%) & 58 \\
GMD (kg/an/día) & 1,121 \\
Producción PV (kg/ha) & 570 \\
\hline
\end{tabular}

Nota: $P C=$ proteína cruda; $\mathrm{DMO}=$ digestibilidad de la materia orgánica; GMD = ganancia media diaria; $\mathrm{kg} / \mathrm{an} / \mathrm{d}$ ía = kilos por animal por día; $\mathrm{PV}=$ peso vivo.

el contenido de proteína cruda descendió hasta $8 \%$, la DMO fue de 50,7 \% y las ganancias mínimas registradas en todo el período fueron 0,375 kg/an/día.

En Río Grande do Sul, Brasil, Neumann et al. (2005) realizaron una experiencia con novillos cruza de las razas Charolais y Nelore, de edad inicial promedio 13 meses y peso inicial promedio de $296 \mathrm{~kg} \mathrm{PV}$, los que pastoreaban sobre un cultivo de sorgo forrajero (Sorghum bicolor). El período de evaluación se extendió desde el 31 de diciembre hasta el 26 de marzo. El Cuadro 11 presenta algunos resultados obtenidos de esta experiencia.

En un ambiente similar al del ensayo anterior, Devicenzi (2011) evaluó el engorde de novillos sobre sorgo forrajero. Los animales pertenecían a la raza Aberdeen Angus, con aproximadamente 20 meses al inicio del ensayo y un PV promedio de $354 \mathrm{~kg}$. Los registros se realizaron desde el mes de febrero, ajustándose una asignación de forraje del
Cuadro 12. Performance animal y calidad de canal y carne de novillos británicos pastoreando sobre sorgo forrajero (adaptado de Devicenzi, 2011).

\begin{tabular}{|lc|}
\hline Parámetro medido & Dato \\
\hline PV faena (kg) & 458 \\
GMD (kg/an/día) & 0,824 \\
Rendimiento (\%) & 49,0 \\
PCC (kg) & 226 \\
PCF $(\mathrm{kg})$ & 222 \\
EGS $(\mathrm{mm})$ & 2,2 \\
AOB $\left(\mathrm{cm}^{2}\right)$ & 63,6 \\
pH & 5,8 \\
Color * L & 34,8 \\
Color * a & 21,2 \\
Color * b & 7,5 \\
CRA $(\%)$ & 68,8 \\
Terneza $\left(\mathrm{kg} \mathrm{F/cm}{ }^{2}\right)$ & 5,5 \\
\hline
\end{tabular}

Nota: $\mathrm{PV}=$ peso vivo; GMD = ganancia media diaria; kg/an/día = kilos por animal por día; Rendimiento = rendimiento carnicero; $\mathrm{PCC}=$ peso canal caliente; $\mathrm{PCF}=$ peso canal fría; $\mathrm{EGS}=$ espesor de grasa subcutánea; $\mathrm{AOB}=$ área ojo de bife; $\mathrm{pH}=\mathrm{pH}$ a las 24 horas post-faena; $\mathrm{CRA}=$ capacidad retención de agua; $\mathrm{kgF} / \mathrm{cm}^{2}=$ kilos de fuerza por centímetro cuadrado.

$13 \%$ PV intentando siempre lograr una altura de frente de forraje ofrecido de $90 \mathrm{~cm}$ y un remanente de $30 \mathrm{~cm}$. Se presentan algunos resultados de este ensayo en el Cuadro 12.

Fernández Mayer et al. (2009) realizaron una experiencia durante dos años con novillos Aberdeen Angus en Argentina. Durante el primer año, se utilizaron dos tandas de animales, con novillos en terminación avan-

Cuadro 11. Características de cultivo sorgo forrajero y performance animal de novillos de razas carniceras (adaptado de Neumann et al., 2005)

\begin{tabular}{|ccccc|}
\hline Parámetro medido & \multicolumn{3}{c}{ Ciclo pastoreo } & Promedio-Total \\
\cline { 2 - 4 } & $\mathbf{1}$ & $\mathbf{2}$ & $\mathbf{3}$ & \\
\hline PC (\%) & 9,9 & 7,5 & 7,2 & 8,2 \\
DMO (\%) & 50,4 & 43,0 & 38,8 & 44,2 \\
Carga total (kg PV/ha) & 2733 & 1209 & 1320 & 1754 \\
GMD (kg/an/día) & 0,495 & 0,680 & 0,658 & 0,608 \\
Producción PV (kg/ha) & 117 & 71 & 78 & 266 \\
\hline
\end{tabular}

Nota: $\mathrm{PC}=$ proteína cruda; $\mathrm{DMO}=$ digestibilidad de la materia orgánica; $\mathrm{GMD}$ = ganancia media diaria; $\mathrm{kg} / \mathrm{an} / \mathrm{día}=$ kilos por animal por día; $\mathrm{PV}=$ peso vivo. 
zada (PV inicial $=406 \mathrm{~kg}$ ) y con novillos en terminación menos avanzada ( $P V$ inicial $=368$ $\mathrm{kg}$ ). Se realizaron tres ciclos de pastoreo y la regulación de la carga se hizo utilizando animales volantes, buscando mantener la utilización de la pastura en el entorno del $65 \%$.

En cuanto a la calidad del sorgo de pastoreo utilizado -tipo BMR azucarado-, en ambos años, el promedio de calidad del forraje fue el siguiente: el máximo de PC se registró en el primer pastoreo $(19,6 \%)$ y el mínimo en el tercer pastoreo $(8,69 \%)$, la DMO se ubicó en el rango entre $78,8 \%$ (primer pastoreo) y $74,9 \%$ (segundo pastoreo), mientras que la FDN registró su máximo en el primer pastoreo $(57,6 \%)$ y su mínimo en el segundo pastoreo (64,3 $\%$ ). En esta oportunidad (segundo año de evaluación), el primer pastoreo se inició más tarde en relación a la altura óptima de pastoreo, a pesar de lo cual el forraje presentó muy buena calidad.

En cuanto a la performance animal del primer año, los novillos iniciaron el ensayo con $406 \mathrm{~kg}$ PV y registraron ganancias de $0,672 \mathrm{~kg} / \mathrm{an} / \mathrm{d}$ ía, mientras que los novillos que iniciaron con $368 \mathrm{~kg} P V$ registraron ganancias de $0,600 \mathrm{~kg} / \mathrm{an} / \mathrm{día}$. Durante el segundo año de evaluación, del primer al segundo pastoreo se lograron ganancias de 1,300 $\mathrm{kg} / \mathrm{an} / \mathrm{día}$ y del segundo al tercer ciclo de pastoreo se obtuvieron ganancias promedio de 0,632 kg PV.
Por otra parte, Fernández et al. (2011ab) continuaron la línea de investigación recién presentada y realizaron otro ensayo de características similares. En esta oportunidad, se utilizaron novillos Aberdeen Angus con un $P V$ inicial de $364 \mathrm{~kg}$, sobre un verdeo de sorgo forrajero tipo BMR fotosensible. La carga animal promedio fue de 4,5 animales/ha equivalente a $1764 \mathrm{~kg} \mathrm{PV/ha} \mathrm{y} \mathrm{la} \mathrm{productividad}$ del sorgo fue de $5800 \mathrm{~kg} \mathrm{MS} / \mathrm{ha}$ en el período considerado, lo que determinó una asignación forrajera del 4,6\% del PV. Se registraron consumos promedio de 10,4 kg $\mathrm{MS} / \mathrm{cab} /$ día y la calidad del forraje fue de $76,9 \%$ de DMO, $12,9 \%$ de PB y $62,5 \%$ de FDN. El contenido de proteína inicial tuvo un nivel de 19,6\%, para luego pasar a 10,4\% y finalmente 8,7\%; estos dos últimos valores se consideran insuficientes para cubrir los requerimientos de estos animales (Dimarco, 1994, citado por Fernández et al., 2011). Las ganancias promedio fueron de 0,801 $\mathrm{kg} / \mathrm{an} /$ día con una producción de peso vivo por unidad de superficie de $249 \mathrm{~kg} \mathrm{PV/ha.}$

En Paraguay, Pusineri y Ocampos (2013) evaluaron el desempeño productivo de novillos sangre cebú y novillos cruza Bos taurus $x$ Bos indicus sobre sorgo forrajero (Sorghum bicolor). Los animales se encontraban en fase terminación, promediando los 27 meses de edad y con $406 \mathrm{~kg}$ PV de peso inicial. El ensayo totalizó 94 días de período de evaluación. El Cuadro 13 presenta algunos resultados de esta experiencia.

Cuadro 13. Evaluación de desempeño de novillos pastoreando sobre sorgo forrajero híbrido (adaptado de Pusineri y Ocampos, 2013).

\begin{tabular}{|lccc|}
\hline Parámetro medido & \multicolumn{3}{c|}{ Ciclo pastoreo } \\
\cline { 2 - 4 } & $\mathbf{1}$ & $\mathbf{2}$ & $\mathbf{3}$ \\
\hline Disponibilidad (kg MS/ha) & 15.803 & 18.585 & 12.485 \\
Utilización (\%) & 68,7 & 65,7 & 70,6 \\
PC (\%) & 13,4 & 10,8 & 14,3 \\
Relación hoja/tallo & 0,96 & 0,64 & 1,12 \\
PV final (kg PV) & & 473 & \\
GMD (kg/an/día) & \multicolumn{3}{c}{0,704} \\
Rendimiento (\%) & 54,2 \\
Nota: kg MS/ha = kilos de materia seca por hectárea; PC = proteína cruda; PV = peso \\
vivo; GMD = ganancia media diaria; kg/an/día = kilos por animal por día; Rendimiento = \\
rendimiento carnicero.
\end{tabular}




\subsubsection{Con suplementación}

En Nebraska, Estados Unidos, Schwarz et al. (2008) realizaron un ensayo durante 72 días, con novillos sobre dos tipos de rastrojo de sorgo: sorgo híbrido ( $\mathrm{T}$ ) y sorgo similar pero conteniendo el gen BMR. Los novillos iniciaron el ensayo con $249 \mathrm{~kg}$ PV a una carga de 2,6 novillos/ha. Los animales se suplementaban diariamente a razón de 1,1 $\mathrm{kg} / \mathrm{cabeza} / \mathrm{día}$ con un suplemento a base de granos de destilería y otros ingredientes, incluyendo un promotor de crecimiento (alimento energético-proteico). En el Cuadro 14 se presentan algunos resultados de esta experiencia.

Cuadro 14. Efecto del pastoreo de rastrojo de sorgo sobre novillos en crecimiento suplementados (adaptado de Schwarz et al., 2008).

\begin{tabular}{|lcc|}
\hline Parámetro medido & T & BMR \\
\hline PV inicial $(\mathrm{kg})$ & \multicolumn{2}{c|}{249} \\
PV final (kg) & $280^{\mathrm{b}}$ & $296^{\mathrm{a}}$ \\
GMD (kg/an/día) & $0,340^{\mathrm{b}}$ & $0,558^{\mathrm{a}}$ \\
\hline
\end{tabular}

Nota: $\mathrm{PV}=$ peso vivo; $\mathrm{GMD}$ = ganancia media diaria; $\mathrm{kg} / \mathrm{an} / \mathrm{día}$ = gramos por animal por día; Valores de una misma línea evaluado con letras distintas son significativamente distintas $(P<0,01)$.

También en Nebraska, Geiss et al. (2010) realizaron un ensayo por 65-69 días repetido en dos años, en el que novillos pastoreaban directamente un rastrojo de cultivo de sorgo híbrido $(T)$ o un sorgo similar pero conteniendo el gen BMR, a una carga de 2,58 novillos/ha en ambos casos. Además, ambos tratamientos eran suplementados a razón de $1,1 \mathrm{~kg} / \mathrm{cabeza} / \mathrm{día}$ con el mismo suplemento que en el ensayo anterior (energético-proteico). En el Cuadro 15 se presentan algunos resultados de esta experiencia.

Al igual que en la experiencia realizada por Schwarz et al. (2008), el uso del sorgo BMR resultó en mejor performance animal que el testigo. No se registraron interacciones entre los tratamientos para ninguno de los parámetros presentados.
Cuadro 15. Efecto del pastoreo de rastrojo de sorgo sobre novillos en crecimiento suplementados (adaptado de Geiss et al., 2010).

\begin{tabular}{|lcc|}
\hline Parámetro medido & T & BMR \\
\hline PV inicial $(\mathrm{kg})$ & 240 & 239 \\
PV final $(\mathrm{kg})$ & $271^{\mathrm{b}}$ & $280^{\mathrm{a}}$ \\
GMD (kg/an/día) & $0,467^{\mathrm{b}}$ & $0,630^{\mathrm{a}}$ \\
\hline
\end{tabular}

Nota: PV = peso vivo; GMD = ganancia media diaria; $\mathrm{kg} / \mathrm{an} / \mathrm{día}=$ gramos por animal por día; Valores de una misma línea evaluado con letras distintas son significativamente distintas $(P<0,01)$.

\subsubsection{Experiencias nacionales}

\subsubsection{Asociaciones entre altura y disponibilidad de forraje en sorgos}

Montossi et al. (2013) encontraron valores asociativos de 312 y $211 \mathrm{~kg} \mathrm{MS} / \mathrm{ha}$ por cada $\mathrm{cm}$ de aumento en la altura del forraje en el cv. LE Comiray, para pre y post pastoreo con valores de exactitud que variaron entre $73 \%$ y $75 \%$, respectivamente (Figura 2).

Asimismo, Montossi et al. (comp. pers), citados por Montossi et al. (2013), registra-
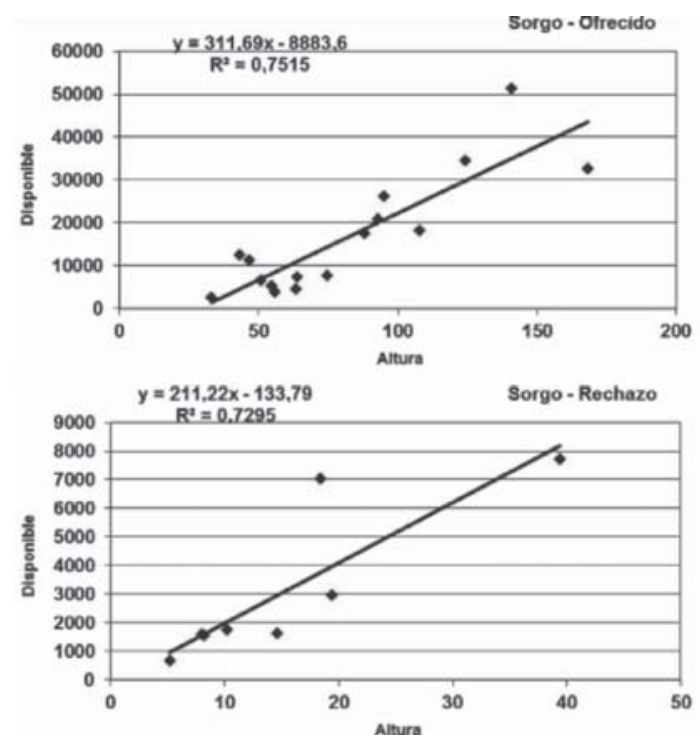

Figura 2. Relación entre altura (cm) y disponibilidad (kg MS/ha) para forraje ofrecido y rechazado de sudangrás (Montossi et al., 2013). 

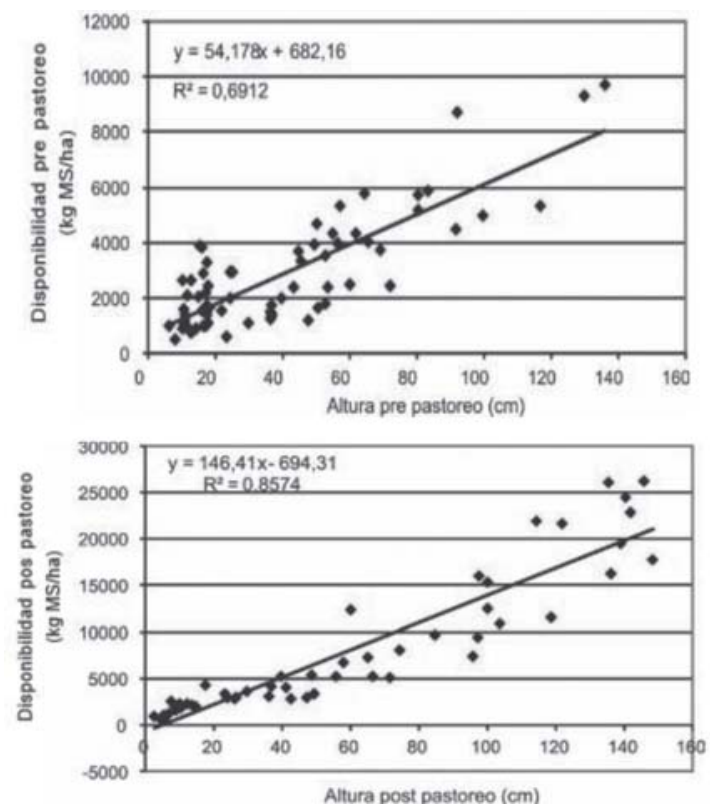

Figura 3. Relación entre altura $(\mathrm{cm})$ y disponibilidad (kg MS/ha) para forraje ofrecido y rechazado de sudangrás (Montossi et al. comp. pers., citados por Montossi et al., 2013).

ron valores de 54 y $146 \mathrm{~kg} \mathrm{MS} / \mathrm{ha}$ por cada $\mathrm{cm}$ de aumento en el mismo cultivar de sudangrás para pre y post pastoreo respectivamente con exactitudes de $69 \%$ y $86 \%$ (Figura 3). En esta experiencia la pastura fue manejada siempre por debajo de los $45 \mathrm{~cm}$.

Rovira (2002) encontró una asociación simple y positiva entre la altura del cultivo y la disponibilidad de materia seca, con un alto coeficiente de correlación $(r=0,88)$. En este caso, un $77 \%$ de la variación de la disponi-

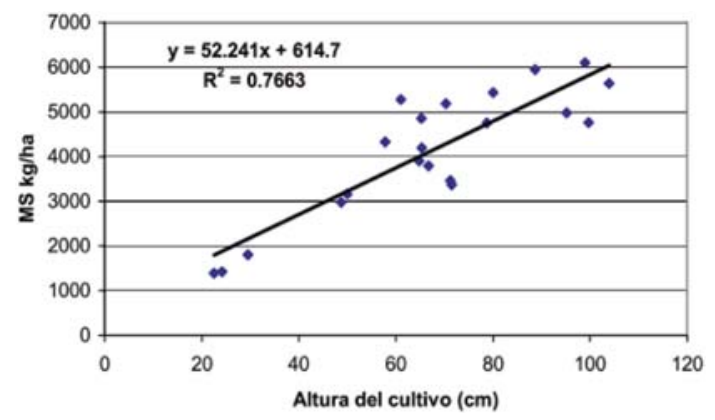

Figura 4. Relación entre altura $(\mathrm{cm})$ y disponibilidad (kg MS/ha) del forraje (Rovira, 2002). bilidad de materia seca estuvo explicada por la altura del tapiz. La ecuación de predicción encontrada entre disponibilidad y altura de forraje se presenta en la Figura 4.

Gabard y Russi (2005) reportan un coeficiente de regresión de $R^{2}=0,88$ entre altura y disponibilidad de forraje pos pastoreo, donde por cada $\mathrm{cm}$ de aumento en la altura del forraje remanente, la materia seca disponible aumentó $63 \mathrm{~kg} \mathrm{MS} / \mathrm{ha}$ en promedio para los tres pastoreos que involucraron este experimento.

Berlangeri (2008) calculó la correlación entre la disponibilidad del forraje y la altura disponible. La Figura 5 presenta los resultados de este cálculo.

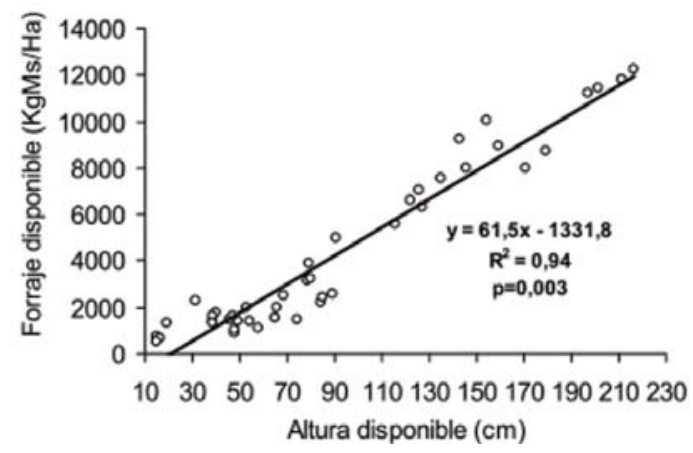

Figura 5. Relación entre disponibilidad de forraje (kg MS/ha) y altura disponible (cm) para todo el período (Berlangeri, 2008).

Como se puede apreciar, se encontró una correlación alta y positiva entre disponibilidad y altura, determinando que por cada $\mathrm{cm}$ de aumento de altura, aumentó el forraje disponible promedio en $61,4 \mathrm{~kg} \mathrm{MS} / \mathrm{ha}$.

Rovira y Echeverría (2013) encontraron una correlación alta y positiva $(P<0,05)$ entre la altura del tapiz y el forraje disponible al inicio de los pastoreos tanto para sudangrás como para sorgo BMR (en ambos casos, $R^{2}=0,80$ ). El modelo de regresión lineal entre altura y disponibilidad también fue significativo $(P<0,05)$, en donde cada $\mathrm{cm}$ adicional de altura correspondió a un incremento de 77 y $102 \mathrm{~kg}$ MS en sudangrás $\left(R^{2}=0,64\right)$ y sorgo $\operatorname{BMR}\left(R^{2}=0,60\right)$, respectivamente. 


\subsubsection{Productividad de pasturas y animales sobre sorgos}

Rovira (2002) realizó una experiencia con novillos de 2 años cruza Hereford $x$ Aberdeen Angus en terminación sobre un verdeo de sudangrás cv. LE Comiray. Los animales comenzaron el ensayo a una dotación de 5,3 animales/ha y con peso inicial de $400 \mathrm{~kg}$ PV. En el Cuadro 16 se presentan los resultados medidos en la pastura.

Cuadro 16. Caracterización de la pastura utilizada por novillos en terminación (adaptado de Rovira, 2002).

\begin{tabular}{|lcc|}
\hline $\begin{array}{l}\text { Parámetros } \\
\text { medidos }\end{array}$ & $\begin{array}{c}\text { Dispo- } \\
\text { nible }\end{array}$ & $\begin{array}{c}\text { Recha- } \\
\text { zos }\end{array}$ \\
\hline $\begin{array}{l}\text { Disponibilidad } \\
\text { (kg MS/ha) }\end{array}$ & 4366 & 2031 \\
Altura (cm) & 74 & 30 \\
Hojas (\%) & 37 & 17 \\
Utilización (\%) & 53,5 & \\
\hline
\end{tabular}

Nota: $\mathrm{kg}$ MS/ha = kilos de materia seca por hectárea.

Cuadro 17.Caracterización de la pastura utilizada por novillos en terminación (adaptado de Rovira, 2002).

\begin{tabular}{|lc|}
\hline Parámetro medido & Dato \\
\hline PV inicial (kg) & 400 \\
PV final (kg) & 424 \\
Carga total (kg PV/ha) & 2197 \\
Carga total (UG/ha) & 5,5 \\
Carga instantánea (kg PV/ha) & 8788 \\
Carga instantánea (UG/ha) & 22 \\
GMD (kg/an/día) & 0,451 \\
Producción PV (kg/ha) & 128 \\
\hline
\end{tabular}

Nota: $\mathrm{PV}=$ peso vivo; $1 \mathrm{UG}=400 \mathrm{~kg} \mathrm{PV} ; \mathrm{GMD}=$ ganancia media diaria; $\mathrm{kg} / \mathrm{an} / \mathrm{d}$ ía = kilos por animal por día.

En el Cuadro 17 se presentan los resultados de producción animal.

La Figura 6 muestra la evolución de las ganancias medias diarias y la relación hoja/tallo entre los distintos ciclos de pastoreo.
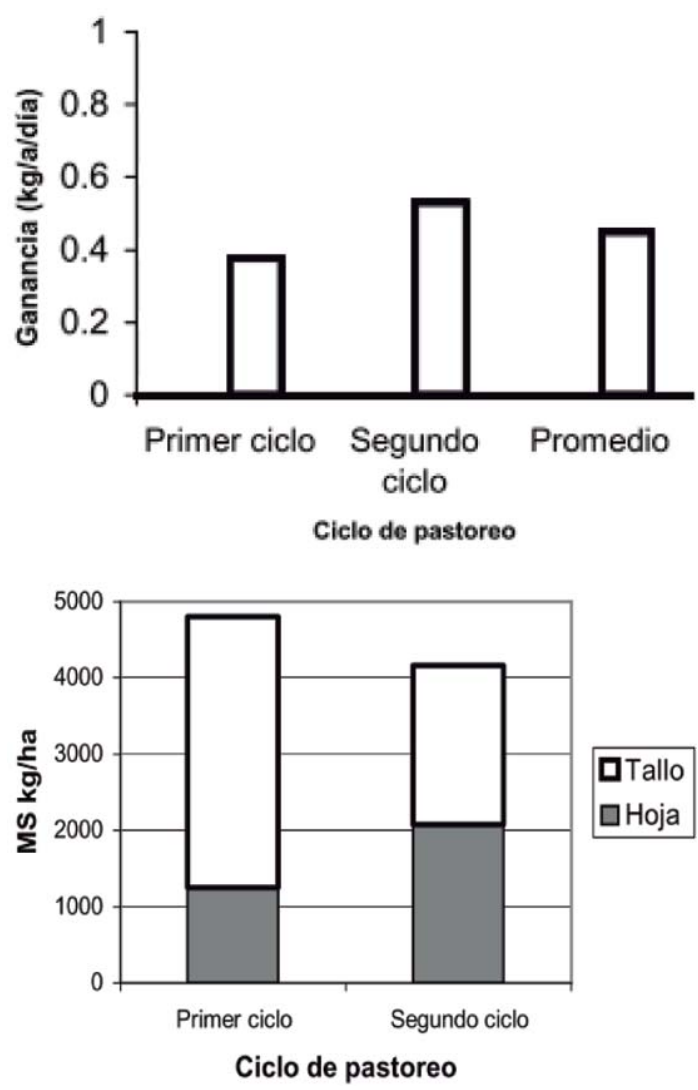

Figura 6. Ganancias medias diarias según ciclo de pastoreo y relación hoja/taIlo (adaptado de Rovira, 2002).

La explicación del incremento de las ganancias del primer ciclo hacia el segundo, independientemente del tratamiento, podría estar fundamentada en el aumento de la relación hoja/tallo en el forraje ofrecido, ya que la disponibilidad fue similar en ambos ciclos.

Gabard y Russi (2005) llevaron a cabo un experimento en el que novillos de raza Holando (2-2,5 años de edad) con un PV inicial de $382 \mathrm{~kg}$ pastoreaban un verdeo de sudangrás cv. LE Comiray, en el que se evaluaron 4 alturas de forraje remanente $(15,30$, 45 y $60 \mathrm{~cm}$ de altura). Se realizaron 3 pastoreos en total, entre el 17 de enero y el 28 de marzo. En el Cuadro 18 se resumen algunos resultados de esta experiencia.

Esquivel et al. (2006) y Velazco et al. (2008) publicaron los resultados de un ensayo de novillos pastoreando sudangrás durante el verano, cuyo objetivo era evaluar el impacto de la sombra en la performance de 
Cuadro 18. Efecto de la altura del remanente sobre la pastura y su utilización (adaptado de Gabard y Russi, 2005).

\begin{tabular}{|cccc|}
\hline $\begin{array}{c}\text { Remanente } \\
(\mathbf{c m})\end{array}$ & $\begin{array}{c}\text { Disponibilidad } \\
\text { (kg MS/ha) }\end{array}$ & $\begin{array}{c}\text { Relación } \\
\text { hoja/tallo }\end{array}$ & $\begin{array}{c}\text { Utilización } \\
\text { (\%) }\end{array}$ \\
\hline 60 & $8115^{\mathrm{a}}$ & $0,35^{\mathrm{c}}$ & $44^{\mathrm{c}}$ \\
45 & $7011^{\mathrm{ab}}$ & $0,37^{\mathrm{bc}}$ & $50^{\mathrm{c}}$ \\
30 & $6229^{\mathrm{bc}}$ & $0,39^{\mathrm{b}}$ & $60^{\mathrm{b}}$ \\
15 & $5223^{\mathrm{c}}$ & $0,43^{\mathrm{a}}$ & $72^{\mathrm{a}}$ \\
\hline
\end{tabular}

Nota: $\mathrm{kg}$ MS = kilos de materia seca; Valores de una misma línea evaluado con letras distintas son significativamente distintas $(P<0,05)$.

novillos en engorde. Se presentan solamente los resultados del tratamiento testigo, sin acceso a sombra. El período experimental fue de 68 días en total (fines de enero a mediados de marzo) y se utilizó el cv. INIA Surubí sembrado a mitad de noviembre. Los animales eran novillos cruza Hereford $x$ Aberdeen Angus, con un PV inicial de 278 $\mathrm{kg}$. El primer pastoreo se atrasó determinando un excesivo crecimiento inicial del forraje, afectando el manejo posterior del verdeo. Algunos resultados se presentan en el Cuadro 19.
En cuanto al comportamiento animal, se reportaron 540 minutos diarios en promedio para la actividad de pastoreo.

Berlangeri (2008) realizó un ensayo con novillos de la raza Holando (PV inicial $=330$ $\mathrm{kg}$ ), contrastando dos cultivares de sorgo forrajero, uno de los cuales era sorgo híbrido (Sorghum sudanense $\times$ Sorghum bicolor) (SG) y el otro BMR. El período experimental se extendió desde noviembre a abril, si bien los animales solo pastorearon en total 90 días. Se evaluaron los dos materiales forrajeros y dos remanentes pos pastoreo (con

Cuadro 19. Resultados en la pastura de novillos pastoreando sudangrás, según ciclo de pastoreo y performance animal (adaptado de Esquivel et al., 2006).

\begin{tabular}{|lccc|}
\hline Parámetros medidos & 1er ciclo & 2do ciclo \\
\hline \hline Carga animal (animales/ha) & & 7 & \\
Disponible ofrecido (kg MS/ha) & 6084 & 4309 \\
Altura disponible ofrecido (cm) & 165 & 113 \\
Utilización (\%) & 36 & 45 \\
DMO (\%) & 59,2 & 59,4 \\
PC (\%) & 7,8 & & 7,5 \\
FDA (\%) & 46,6 & & 42,0 \\
GMD (kg/an/día) & & 0,473 & \\
PV final (kg) & & 312 & \\
Producción PV (kg/ha) & \multicolumn{3}{|c}{272} \\
\hline
\end{tabular}

Nota: $\mathrm{kg}$ MS = kilos de materia seca; $\mathrm{DMO}$ = digestibilidad de la materia orgánica; $\mathrm{PC}=$ proteína cruda; FDA = fibra detergente ácido; $\mathrm{GMD}$ = ganancia media diaria; $\mathrm{kg} / \mathrm{an} / \mathrm{d} i ́ \mathrm{a}=$ kilos por animal por día; PV = peso vivo. 
Cuadro 20. Efecto del tipo de sorgo forrajero y el manejo del remanente de forraje con novillos Holando (adaptado de Berlangeri, 2008).

\begin{tabular}{|c|c|c|c|c|}
\hline Parámetros medidos & BMR-R & BMR-SR & SG-R & SG-SR \\
\hline Carga instantánea promedio (UG/ha)* & 20,0 & 17,7 & 20,0 & 19,0 \\
\hline AF (\%) & 7,3 & 5,9 & 6,9 & 6,5 \\
\hline Disponible ofrecido ( $1^{\mathrm{er}}$ pastoreo) (kg MS/ha) & 11.152 & 9.918 & 9.939 & 10.727 \\
\hline Disponible ofrecido ( $2^{\text {do }}$ y $3^{\text {er }}$ pastoreo) $(\mathrm{kg} \mathrm{MS} / \mathrm{ha})$ & $2378^{a}$ & $1118^{b}$ & $2157^{a b}$ & $1276^{a b}$ \\
\hline Altura disponible ofrecido promedio $(\mathrm{cm})$ & 91 & 76 & 92 & 83 \\
\hline Altura disponible rechazo promedio $(\mathrm{cm})$ & $36^{b}$ & $14^{\mathrm{a}}$ & $37^{b}$ & $14^{\mathrm{a}}$ \\
\hline GMD (kg/an/día) & 0,930 & 0,760 & 0,850 & 0,740 \\
\hline Producción PV (kg/ha) & 370 & 359 & 400 & 288 \\
\hline
\end{tabular}

Nota: * 1 UG= 400 kg PV; PV = peso vivo; GMD = ganancia media diaria; g/an/día = gramos por animal por día; $A F=$ asignación diaria de forraje; Valores de una misma línea evaluado con letras distintas son significativamente distintas $(P<0,05)$.

rotativa: $\mathrm{R}$ y sin rotativa: $\mathrm{SR})$. Se presentan algunos resultados de esta experiencia en el Cuadro 20.

Los porcentajes de utilización del forraje no difirieron entre tratamientos $(P<0,05)$, promediando $52,5 \%$ (máximo $=58 \%$ y mínimo $=48 \%)$. No se encontró ninguna relación entre la altura del forraje disponible y el porcentaje de utilización del forraje ofrecido. En cuanto a la calidad del forraje ofrecido, el porcentaje de PC de los SG oscilaron entre 12,7 y $16,7 \%$, mientras que para el BMR los contenidos se situaron entre 12,6 y $13,0 \%$.

Al comparar la producción de PV/ha en todo el período, se encontró diferencia estadísticamente significativa $(P<0,05)$ entre los cultivares de sorgo (BMR = $365 \mathrm{~kg} \mathrm{PV/ha;}$ $\mathrm{SG}=344 \mathrm{~kg}$ PV/ha).

Vaz Martins et al. (2003 y 2001) y Vaz Martins (2000) llevaron adelante una experiencia con novillos Hereford pastoreando tres tipos de sorgos: sudangrás cV. LE Comiray y dos híbridos Sorghum bicolor x Sorghum sudanense ( $\mathrm{Sg} \mathrm{H} 1$ y Sg H2). El objetivo era evaluar dos alturas contrastantes de ingreso a los verdeos: $60 \mathrm{~cm}$ y $100 \mathrm{~cm}$. La altura del remanente en todos los casos fue en promedio $15 \mathrm{~cm}$. En el Cuadro 21 se resume la información relacionada a la pastura.

Los días de pastoreo fueron mayores en aquellos materiales de manejo a $60 \mathrm{~cm}$ de altura y, como consecuencia, el número de pastoreos también fue mayor que en el caso del manejo a $100 \mathrm{~cm}$ de altura. En cuanto a los resultados de performance animal, el Cuadro 22 presenta alguno de los resultados de este ensayo.

No se encontraron diferencias $(P<0,05)$ de ganancia de peso vivo entre tipos de sorgo ni para la interacción de manejos por tipo de sorgos, pero sí entre manejos. No se observó ningún síntoma de toxicidad por ácido cianhídrico a lo largo del período experimental.

En este caso, el sudangrás fue el tipo de sorgo más apropiado para pastoreo por capacidad de carga, calidad de forraje y días de pastoreo, que determinó mayores ganancias de peso vivo por animal y por unidad de superficie.

Rovira y Echeverría (2013) realizaron un ensayo con novillos para contrastar dos bases forrajeras: sudangrás (Sorghum sudanense cV. LE Comiray) y sorgo forrajero (Sorghum vulgare cv. AGT 21, tipo BMR). Los novillos eran de 1,5 años de edad cruza Hereford $x$ Aberdeen Angus (PV inicial $=304$ $\mathrm{kg}$ ). El Cuadro 23 presenta las características del forraje disponible de los verdeos, según ciclo de pastoreo.

En cuanto al forraje remanente, no se registraron diferencias significativas $(P<0,05)$ entre tratamientos en la altura y disponibilidad de forraje durante los tres ciclos de pastoreo. 
Cuadro 21. Caracterización de distintos tipos de sorgo (sudán y sorgos híbridos) como base forrajera para novillos en crecimiento (adaptado de Vaz Martins et al., 2003).

\begin{tabular}{|c|c|c|c|c|c|c|}
\hline \multirow[t]{2}{*}{ Parámetros medidos } & \multicolumn{3}{|c|}{$60 \mathrm{~cm}$} & \multicolumn{3}{|c|}{$100 \mathrm{~cm}$} \\
\hline & Sudán & Sg H1 & $\mathrm{Sg} \mathrm{H} 2$ & Sudán & $\mathrm{Sg} \mathrm{H1}$ & $\mathrm{Sg} \mathrm{H} 2$ \\
\hline \multicolumn{7}{|c|}{ Forraje ofrecido } \\
\hline Disponible (kg MS/ha) & 1489 & 1331 & 1549 & 2035 & 2401 & 3339 \\
\hline Altura $(\mathrm{cm})$ & 50 & - & 56 & 74 & 96 & 74 \\
\hline Relación hoja/tallo & 0,84 & 0,74 & 0,96 & 0,70 & 0,55 & 0,64 \\
\hline Utilización (\%) & 49 & 53 & 45 & 42 & 51 & 46 \\
\hline \multicolumn{7}{|c|}{ Forraje remanente } \\
\hline Disponible (kg MS/ha) & 834 & 737 & 1043 & 1238 & 1111 & 1694 \\
\hline Relación hoja/tallo & 0,19 & 0,16 & 0,20 & 0,10 & 0,09 & 0,15 \\
\hline $\mathrm{N}^{\circ}$ pastoreos & 5 & 4 & 3 & 3 & 3 & 2 \\
\hline Días pastoreo & 118 & 107 & 107 & 101 & 92 & 79 \\
\hline
\end{tabular}

Nota: $\mathrm{kg} \mathrm{MS/ha} \mathrm{=} \mathrm{kilos} \mathrm{de} \mathrm{materia} \mathrm{seca} \mathrm{por} \mathrm{hectárea.}$

Cuadro 22. Desempeño de novillos en crecimiento pastoreando distintos tipos de sorgo (sudán y sorgos híbridos) (adaptado de Vaz Martins et al., 2003).

\begin{tabular}{|c|c|c|c|c|c|c|}
\hline \multirow[t]{2}{*}{ Parámetros medidos } & \multicolumn{3}{|c|}{$60 \mathrm{~cm}$} & \multicolumn{3}{|c|}{$100 \mathrm{~cm}$} \\
\hline & Sudán & Sg H1 & $\mathrm{Sg} \mathrm{H} 2$ & Sudán & Sg H1 & $\mathrm{Sg} \mathrm{H} 2$ \\
\hline Carga animal (UG/ha) & 3,4 & 3,0 & 3,5 & 2,4 & 2,2 & 3,0 \\
\hline PV inicial (kg) & 190 & 194 & 191 & 190 & 192 & 192 \\
\hline PV final $(k g)$ & $290^{a}$ & $270^{a}$ & $277^{a}$ & $263^{b}$ & $266^{b}$ & $255^{b}$ \\
\hline GMD (kg/an/día) & 0,888 & 0,849 & 0,980 & 0,718 & 0,788 & 0,803 \\
\hline GMD (kg/an/día) & \multicolumn{3}{|c|}{$0,906^{a}$} & \multicolumn{3}{|c|}{$0,769^{b}$} \\
\hline Producción PV/ha (kg/ha) & 570 & 395 & 507 & 314 & 321 & 367 \\
\hline
\end{tabular}

Nota: UG/ha = unidad ganadera por hectárea; PV = peso vivo; GMD = ganancia media diaria; kg/an/día = kilo por animal por día; Valores de una misma línea evaluado con letras distintas son significativamente distintas $(P<0,05)$.

En el Cuadro 24 se presenta el desempeño productivo de los novillos.

Como se puede observar, a pesar que no existieron diferencias significativas $(P<0,05)$ en el PV final de los animales, se lograron ganancias mayores $(P<0,05)$ con el sorgo forrajero BMR que con el sudangrás.

Desde el punto de vista nutricional, el principal parámetro responsable del descenso en el desempeño productivo de los animales fue el contenido de proteína cruda, la cual disminuyó al avanzar el ciclo de producción. La oferta total de proteína cruda se consideró limitante para un adecuado crecimiento de los animales al tercer ciclo de pastoreo $(12,9 \%$ en hoja y $3,5 \%$ en tallo, siendo que la fracción tallo representó más del 70 \% del forraje). 
Cuadro 23. Caracterización de dos verdeos de verano (sudan y sorgo SG-BMR) como base forrajera para novillos en fase de terminación (adaptado de Rovira y Echeverría, 2013).

\begin{tabular}{|ccrc|}
\hline Pastoreo & Parámetros medidos & Sudan & SG BMR \\
\hline Primero & Disponible ofrecido (kg MS/ha) & 5973 & 6845 \\
& Altura (cm) & 101 & 98 \\
& Hojas (\%) & 50 & 49 \\
\hline Segundo & Disponible ofrecido (kg MS/ha) & 7451 & 9550 \\
& Altura (cm) & 95 & 93 \\
& Hojas (\%) & 25 & 26 \\
\hline Tercero & Disponible ofrecido (kg MS/ha) & 5712 & 6935 \\
& Altura (cm) & 84 & 85 \\
& Hojas (\%) & 24 & 21 \\
\hline
\end{tabular}

Nota: $\mathrm{kg} \mathrm{MS/ha} \mathrm{=} \mathrm{kilos} \mathrm{de} \mathrm{materia} \mathrm{seca} \mathrm{por} \mathrm{hectárea.}$

Cuadro 24. Desempeño de novillos pastoreando sudangrás o sorgo (adaptado de Rovira y Echeverría, 2013).

\begin{tabular}{|lccc|}
\hline \multicolumn{2}{l}{ Parámetros medidos } & Sudan & SG BMR \\
\hline PV inicial $(\mathrm{kg})$ & & 320 & 320 \\
PV final $(\mathrm{kg})$ & & 378 & 388 \\
GMD (kg/an/día) & $0-21$ días & $0,796^{\mathrm{b}}$ & $1082^{\mathrm{a}}$ \\
& $21-42$ días & 1,034 & 1,091 \\
& $42-73$ días & 0,669 & 0,708 \\
& $0-73$ días & $0,823^{\mathrm{b}}$ & $0,945^{\mathrm{a}}$ \\
\hline
\end{tabular}

Nota: $\mathrm{PV}=$ peso vivo; GMD = ganancia media diaria; $\mathrm{kg} / \mathrm{an} / \mathrm{día}=$ kilo por animal por día; Valores de una misma línea evaluado con letras distintas son significativamente distintas $(P<0,05)$.

\section{BIBLIOGRAFÍA}

BERLANGERI, S. 2008. Efecto del manejo y el material genético en la productividad de sorgo forrajero bajo pastoreo. Tesis Ingeniero Agronomo. Montevideo, Uruguay. Facultad de Agronomía. 189 p.

BERTI, R. 2011. Efecto de la suplementacion azufrada sobre la ganancia diaria de bovinos pastoreando sorgos forrajeros hibridos. [En línea]. Buenos Aires: INTA. Consultado: 27 abr. 2015. Disponible en: http://inta.gob.ar/documentos/efectode-la-suplementacion-azufrada-sobrela-ganancia-diaria-de-bovinos-pastoreando-sorgos-forrajeros-hibridos/
BIANCO, A.; ASTIGARRAGA, L.; MELLO, R.; BATTEGAZZORE, G.; MONTEDÓNICO, D. 2003. Estudio de las características de híbridos de sorgo de bajo contenido en lignina $(B M R)$ y la respuesta en leche en pastoreo directo o conservado como ensilaje. [En línea]. Montevideo: Facultad de Agronomía. Consultado $27 \mathrm{abr}$. 2015. Disponible en: http:// www.biscayart.com/images/infotecnica/ sorgobmryrepuestaenleche.pdfg

CARÁMBULA, M. 1964. Efectos de diferentes intensidades y frecuencias de corte en sudangras (Sorghum sudanense). Montevideo: Facultad de Agronomía. 11 p. (Boletín; 7). 
CARÁMBULA, M. 2007. Verdeos de verano. Montevideo: Hemisferio Sur. 226 p.

CASLER, M.; PEDERSEN, J.; UNDERSANDER, D. 2003. Forage yield and economic losses associated with the Brown-Midrib trait in sudangrass. Crop Science, 43: 782-789.

CONTRERAS-GOVEA, F.; MARSALIS, M.; LAURIAULT, L.; BEAN, B. 2010. Forage Sorghum Nutritive Value: A Review. Forage and Grazinglands, 8(1).

COSER, A.; MARASCHIN, G. 1983. Desempenho animal em pastagens de capim-gordura e braquiária. Pesquisa Agropecuaria Brasilera, 18 (4): 21-426.

DEVICENZI, T. 2011. Características da carcaca e da carne de novilhos Aberdeen Angus terminados em diferentes pastagens. MSc Thesis, Universidade Federal do Rio Grande do Sul. 110 p.

DE RUITER, J.; DALLEY, D.; HUGHES, T.; FRASER, T.; DEWHURST, R. 2007. Types of supplements: their nutritive value and use. En: Rattray, P.V.; Brookes, I.M.; Nicol, A.M. (eds.). Pasture and supplements for grazing animals. Hamilton, New Zealand: New Zealand Society of Animal Production. p. 97-115. (Occasional Publication; 14).

ESQUIVEL, J.; VELAZCO, J.; ROVIRA, P. 2006. Efecto del acceso a sombra artificial en la ganancia de peso, estrés y conducta de novillos pastoreando sudangrás durante el verano. En: Jornada Anual Unidad Experimental Palo a Pique. INIA Treinta y Tres. Montevideo: INIA. p 2236. (Serie Actividades de Difusión; 511).

FASSIO, A.; CAZZOLINO, D.; IBAÑEZ, V.; FERNÁNDEZ, E. 2002. Sorgo: destino forrajero, INIA La Estanzuela. Montevideo: INIA. 30 p. (Serie Técnica; 127).

FERNÁNDEZ MAYER, A.; CORIA, M.; CHIATELLINO, D. 2009. Engorde pastoril con sorgos nervadura marrón (Brown Middle Rib - BMR) [En línea]. Consultado: abr. 2015. Disponible en http:// www.produccion-animal.com.arl informacion_tecnica/invernada_o_engorde_pastoril_o_a_campo35nervadura_marron.pdf

FERNÁNDEZ MAYER, A.; STUART, R.; CHONGO, B.; MARTIN, P. 2011. Ceba pastoril con sorgos nervadura marrón o BMR (Brown Middle Rib) como forraje fresco. Revista
Cubana de Ciencia Agrícola, 45(3): $251-$ 256.

FIELD, T. 2007. Beef production and management decisions. Colorado: Colorado State University. $718 \mathrm{p}$.

GABARD, L.; RUSSI, I. 2005. Efecto de la intensidad de pastoreo en producción y dinámica poblacional de Sorghum sudanense var. Comiray. Tesis Ingeniero Agrónomo. Montevideo, Uruguay. Facultad de Agronomía. 100 p.

GALLARINO, H. 2008. Manejo de sorgos forrajeros, su aprovechamiento. Marca Líquida Agropecuaria, 18(180): 52-54.

GEISS, J. R.; WATSON, A.; KLOPFENSTEIN, T.; ERICKSON, G.; BENTON, J. 2010. Forage Quality and Grazing Performance of Beef Cattle Grazing Brown Midrib Grain Sorghum Residue. Nebraska Beef Cattle Reports. [En línea]. Consultado: abr. 2015. Disponible en: http://digital commons.unl.edu/cgi/viewcon-tent.cgi? article $=1559 \&$ context $=$ animalscinbcr

GIORELLO, D.; JAURENA, M.; BOGGIANO, P.; PEREZ GOMAR, E. 2012. Respuesta al riego suplementario en pasturas y forrajes. En: Seminario internacional, riego en cultivos y pasturas, 2., Salto, Uruguay. Montevideo: INIA. p. 45 - 54.

LAGOMARSINO, X.; MONTOSSI, F. 2014. Engorde estival de novillos en pastoreo sobre sorgos forrajeros con suplementación proteica. Revista INIA, 39: 17-22.

LAGOMARSINO, X.; LUZARDO, S.; MONTOSSI, F. 2014. ¿Cómo producir terneros con más de $300 \mathrm{~kg}$ con edades menores a los 15 meses en sistemas ganaderos de Basalto? En: Estrategias de intensificación ganadera, INIA Treinta y Tres. Montevideo: INIA. p. 33-38. (Serie Actividades Difusión; 734).

MC CUISTION, K.; MC COLLUM, F.; GREENE, L.; MAC DONALD, J.; BEANT, B. 2011. Performance of stocker cattle grazing two sorghum-sudangrass hybrids under various stocking rates. The Professional Animal Scientist, 27: 92-100.

MC CUISTON, K.; MC COLLUM, F.; GREENE, L.; MAC DONALD, J.; BEAN, B.; VAN METER, R. 2007. Performance of stocker cattle grazing two sorghum-sudagrass hybrids under various stocking rates. Beef Cattle Research in Texas [En línea]. Consulta- 
do: abr. 2015. Disponible en http:// coals.tamu.edu

MC CUISTION, K.; MC COLLUM, T.; GREENE, W.; BEAN, B.; VAN METER, R.; VASCONCELOS, J.; SILVA, J. 2005. Performance of steers grazing photoperiodsensitive and brown midrib varieties of sorghum-sudangrass. Forage sorghum field day. Amarillo: Texas Agricultural Research and Extension Centre. p. 1416.

MC DONALD, P.; EDWARDS, R.; GREENHALGH, J.; MORGAN, C.; SINCLAIR, L.; WILKINSON, R. 2011. Animal nutrition. London: Pretice Hall. 692 p.

MIERES, J.M. 2004. Guía para la alimentación de rumiantes, INIA La Estanzuela. Montevideo: INIA. 81 p. (Serie Técnica; 142).

MONTOSSI, F.; DE BARBIERI, I.; DIGHIERO, A. 2013. El uso de la altura del forraje: una herramienta disponible para el manejo eficiente de sistemas pastoriles orientados a la producción ovina. En: Montossi, F.; De Barbieri, I. (eds). Tecnologías de engorde de corderos pesados sobre pasturas cultivadas en Uruguay. Montevideo. INIA. p. 159-182. (Serie Técnica; 206).

NEUMANN, M.; RESTLE, J.; FILHO, D.; MACCARI, M.; PELLEGRINI, L.; SOUZA, A.; PEIXOTO, L. 2005. Qualidade de forragem e desempenho animal em pastagem de sorgo (Sorghum bicolor L.) fertilizada com dois tipos de adubo, sob pastejo continuo. Revista Brasilera Agrociencia, 11(2): 221-226.

N. R. C. (National Research Council). 1996. Nutrient requirements of beef cattle. Washington, D. C.: National Academy Press.

PERUCHENA, C. 1999. Suplementación de bovinos para carne sobre pasturas tropicales: aspectos nutricionales, productivos y económicos. [En línea]. Consultado: abr. 2015. Disponible en: http:// www.produccion-animal.com.ar/informa-cion_tecnica/invernada_o_engord e sorgos_bmr.pdf

PERUCHENA, C. 2003. Suplementacion de bovinos en sistemas pastoriles. Proyecto Ganadero Corrientes. [En línea]. Consultado: abr. 2015. Disponible en: http:// www.produccion-animal.com.ar/ informacion_tecnica/suplementacion/ 107-en_siste-mas_pastoriles.pdf

PITTALUGA, P.; BRITO, G.; SOARES DE LIMA, J.; DEL CAMPO, M.; ZAMIT, W.; DA CUNHA, K.; PIÑEIRO, A.; LAGOMARSINO, X.; OLIVERA, J.; TRINDADE, G.; ARRIETA, G.; MOREIRA, R. 2005. Efecto de diferentes dietas sobre el crecimiento carnicero y la calidad de carne. En: Día de campo Producción animal, pasturas y forestal; Unidad Experimental Glencoe, INIA Tacuarembó. Montevideo: INIA. p. 43-50. (Serie Actividades Difusión; 431).

PORDOMINGO, A. 2003. Suplementación con granos a bovinos en pastoreo. [En línea]. Consultado: abr. 2015. Disponible en: http://www.produccion-animal. com.ar/ informacion_tecnica/suplemen-tacion/ 67 -suplementacion_con_granos_en_pastoreo.pdf

PUSINERI, J.; OCAMPOS, D. 2013. Desempeño productivo de novillos cebuinos Bos indicus e híbridos Bos taurus $x$ Bos indicus mantenidos bajo pasatoreo rotativo de sorgo forrajero Sorghum bicolor L. Mench. Investigación Agraria, 6 (2): 2933.

RESTLE, J.; ROSO, C.; AITA, V.; NÖRNBERG, J.; BRONDANI, I.; CERDÓTES, L.; DE OLIVEIRA CARRILHO, C. 2002. Produção animal em pastagem com gramíneas de estação quente. Revista Brasileira de Zootecnia, 31(3): 1491-1500.

RIBAS, M.; MACHADO, F. 2009. Produção de forragem utilizando híbridos de sorgo com capim Sudão (S . bicolor x S . sudanense). [En línea]. Consultado: abr. 2015. Disponible en: http://abms.org.br/ cn_milho/palestras/011.pdf

ROVIRA, P. 2002. Efecto de la sombra artificial en el engorde de novillos durante los meses de verano. En: Jornada anual de producción animal. Resultados experimentales, INIA Treinta y Tres. Montevideo: INIA. p. 79-95. (Serie Actividades de Difusión ; 294).

ROVIRA, P.; ECHEVERRÍA, J. 2013. Desempeño productivo de novillos pastoreando sudangras o sorgo forrajero nervadura marrón (BMR) durante el verano. Revista Veterinaria, 24 (2): 91-96.

SCHWARZ, A.; GODSEY, C.; LUEBBE, M.; ERICKSON, G.; KLOPFENSTEIN, T. 2008. Forage Quality and Grazing Performan- 
ce of Beef Cattle Grazing Brown Mid-rib Grain Sorghum Residue. [En línea]. Consultado: abr. 2015. Disponible en: http:// digitalcommons.unl.edu/cgi/ viewcontent.cgi?article $=1012 \&$ context=animalscinbcr\&sei-redir=1\&referer=http://scholar.google.es/scholar? $\mathrm{hl}=\mathrm{es} \& \mathrm{q}=$ Forage+quality+and+grazing + performance+of+beef+cattle+grazing+ brown+midrib+grain+sorghum+residue.+(en+l?

SILUNGWE, D. 2011. Evaluation of forage yield and quality of sorghum, sudangrass and pearl millet cultivars in Manawatu. MSc. Thesis, Massey (NZ), Massey University. $140 \mathrm{p}$.

VASCONCELOS, J; GREENE, L.; MC COLLUM, F.; BEAN, B.; VAN METER, R. 2003. Performance of crossbred steers grazing photoperiod sensitive and non photoperiod sensitive sorghum sudangrass hybrids. Journal of Animal Science, 81 (Supplemment 2): 1-2.
VAZ MARTINS, D. 2000. Pastoreo de sorgo para engorde de ganado: otra alternativa para el verano. Revista Plan Agropecuario, (94): 31-36.

VAZ MARTINS, D.; SEIGAL, E.; PITTALUGA, O. 2001. Producción de carne con tres tipos de sorgo bajo dos sistemas de manejo. Revista Argentina de Producción Animal, 21 (1): 72-73.

VAZ MARTINS, D.; SEIGAL, E.; PITTALUGA, 0. 2003. Producción de carne con sudangrass dulce, híbrido de sudangrass por sorgo granífero y sorgo doble propósito. En: Vaz Martins, D. (ed.), Avances sobre engorde de novillos en forma intensiva. INIA La Estanzuela. Montevideo: INIA. p. 19-22. (Serie Técnica; 135).

VELAZCO, J.; ROVIRA, P.; ESQUIVEL, J. 2008. Efecto del acceso a sombra artificial en la ganancia de peso de novillos pastoreando sudangrás durante el verano (Uruguay). Revista Argentina de Producción Animal, 28 (1): 237-302. 


\section{INTRODUCCIÓN}

Los resultados que se presentan en este capítulo, se dividen en dos grandes secciones. En la primera sección, se muestran los dos años de evaluación del pastoreo de sorgo forrajero y sudangrás de novillos en proceso de terminación con suplementación proteica (años 2012 y 2013). En la segunda sección, se presentan los dos años de evaluación del pastoreo de sorgo forrajero de novillos con suplementación de diferentes características (años 2014 y 2015), así como los resultados de un año de evaluación de distintas cargas y el uso de animales fistulados para estudios del ambiente ruminal (año 2014).

\section{EVALUACIÓN DE LA}

\section{PRODUCTIVIDAD DE NOVILLOS PASTOREANDO SORGO FORRAJERO Y SUDANGRÁS CON SUPLEMENTACIÓN PROTEICA}

El objetivo general de estos ensayos fue evaluar en forma comparativa el pastoreo del sorgo forrajero azucarado (BMR) y sudangrás (cv. INIA Comiray) conjuntamente con la evaluación de la suplementación proteica, como alternativas para el engorde de novillos durante el período estival en la región de Basalto.

Los objetivos específicos fueron:

- Evaluar la performance animal de novillos Hereford sobre un verdeo de sorgo tipo BMR (cv. Nutritop) a una carga de 7,5 novillos/ha.

- Evaluar la performance animal de novillos Hereford sobre un verdeo de sudangrás (cv. INIA Comiray) a una carga de 7,5 novillos/ha.

- Evaluar el efecto de la suplementación con expeller de girasol en la productividad animal, sobre verdeos de sorgo tipo BMR (cv. Nutritop) y de sudangrás (cv. INIA Comiray).

- Evaluar el efecto del pastoreo y la suplementación en la productividad de verdeos de sorgo tipo BMR (cv. Nutritop) y de sudangrás (cv. INIA Comiray).

- Evaluar la interacción de los factores evaluados.

- Evaluar el efecto de los tratamientos sobre la deposición de tejidos en el animal in vivo.

\subsection{Materiales y métodos}

\subsubsection{Tratamientos}

Este ensayo fue desarrollado en dos años consecutivos. En el primer año, el período experimental comenzó el 19 de diciembre de 2011 y finalizó el 2 de abril de 2012 (105 días) mientras que, en el segundo año, el período experimental comenzó el 8 de enero y finalizó el 7 de marzo de 2013 (58 días).

El Cuadro 1 presenta un esquema de los tratamientos aplicados.

Los animales de estos experimentos provenían de ensayos realizados durante su primer invierno de vida, por lo que la distribución para cada tratamiento estival tuvo en cuenta el tipo de alimentación que recibieron los mismos previamente, de manera de mitigar los efectos nutricionales previos al inicio de cada ensayo.

\footnotetext{
* Ing. Agr. Programa Nacional de Producción de Carne y Lana, INIA Tacuarembó (2011-2015).

** Ing. Agr. MSc. Programa Nacional de Producción de Carne y Lana, INIA Tacuarembó.

*** Ing. Agr. PhD. Programa Nacional de Producción de Carne y Lana, INIA Tacuarembó

$\star * * *$ Ing. Agr. PhD. Director Nacional de INIA.
} 
Cuadro 1. Tratamientos experimentales y peso vivo lleno al inicio del ensayo para los años 2012 y 2013.

\begin{tabular}{|lcccc|}
\hline Tratamiento & $\mathbf{1}$ & $\mathbf{2}$ & $\mathbf{3}$ & $\mathbf{4}$ \\
\hline Nomenclatura & SudanSS & SudanCS & SorgoSs & SorgoCs \\
\hline Pastura & Sudangrás & Sudangrás & Sorgo BMR & Sorgo BMR \\
Suplemento (\% PV) & 0 & 1 & 0 & 1 \\
PV Inicial (kg) - Año 2012 & 313,0 & 313,6 & 313,5 & 313,2 \\
PV Inicial (kg) - Año 2013 & 295,4 & 295,7 & 295,3 & 295,6 \\
\hline
\end{tabular}

SS = sin suplementación; CS = con suplementación.

\subsubsection{Materiales y métodos}

El Cuadro 2 presenta el manejo previo y durante la siembra de los verdeos, según el año de evaluación.
Se trabajó con 40 novillos de la raza Hereford, de 15 meses de edad promedio al inicio del período experimental, nacidos en la primavera de los años 2010 y 2011, para el año 1 y año 2 , respectivamente.

Cuadro 2. Manejo previo y durante la siembra de los verdeos según año de evaluación.

\begin{tabular}{|c|c|c|}
\hline Labor & Año 1 & Año 2 \\
\hline $\begin{array}{l}\text { Acondicionamiento } \\
\text { previo }\end{array}$ & $\begin{array}{l}\text { 04/10/11: } 3,5 \text { L/ha de } \\
\text { glifosato } \\
\text { 04/10/11: atrazina (2,9 L/ha) } \\
\text { y metolaclor (Dual Gold, } \\
\text { 1,4 L/ha) }\end{array}$ & $\begin{array}{l}\text { 25/09/12 y } 30 / 09 / 2012: 3,0 \mathrm{~L} / \mathrm{ha} \\
\text { de glifosato. }\end{array}$ \\
\hline Material genético & \multicolumn{2}{|c|}{$\begin{array}{l}\text { Sorgo BMR cv. Nutritop } \\
\text { Sudangrás cv. LE Comiray }\end{array}$} \\
\hline $\begin{array}{l}\text { Tratamiento } \\
\text { de la semilla }\end{array}$ & $\begin{array}{l}\text { Protector (Concep: Fluxofenim) } \\
\text { 4cc/10kg semilla } \\
\text { Insecticida (Imidacloprid 60\% } \\
\text { FS) } 350 \mathrm{cc} / 100 \mathrm{~kg} \text { semilla } \\
\text { Fungicida (Metalaxil } 35 \% \text { ) } \\
350 \mathrm{cc} / 100 \mathrm{~kg} \text { semilla }\end{array}$ & $\begin{array}{l}\text { Protector (Concep: Fluxofenim) } \\
\text { 4cc/10kg semilla } \\
\text { Insecticida (Imidacloprid } 60 \% \\
\text { FS) } 350 \mathrm{cc} / 100 \mathrm{~kg} \text { semilla }\end{array}$ \\
\hline Método de siembra & Directa, a $32 \mathrm{~cm}$ entre hilera. & Directa, a $37 \mathrm{~cm}$ entre hilera. \\
\hline Densidad de siembra & \multicolumn{2}{|c|}{$\begin{array}{l}\text { Sorgo BMR: } 25 \mathrm{~kg} / \mathrm{ha} \\
\text { Sudangrás: } 25 \mathrm{~kg} / \mathrm{ha}\end{array}$} \\
\hline Fertilización basal & $120 \mathrm{~kg} / \mathrm{ha}$ de 25.33 .0 & $100 \mathrm{~kg} / \mathrm{ha}$ de 25.33 .0 \\
\hline Fecha de siembra & $\begin{array}{l}\text { 18/10/2011: Sorgo BMR } \\
\text { 19/10/2011: Sudangrás }\end{array}$ & 01/11/2012: Sorgo BMR y Sudangrás \\
\hline Resiembra & No fue necesaria & $\begin{array}{l}\text { * 20/11/12: Resiembra Sorgo BMR } \\
\text { y Sudangrás }\end{array}$ \\
\hline Refertilización & $\begin{array}{l}50 \mathrm{~kg} / \mathrm{ha} \text { de urea: Sorgo BMR } \\
\text { 18/10/11 } \\
50 \mathrm{~kg} / \mathrm{ha} \text { de urea: Sudangrás } \\
19 / 10 / 11\end{array}$ & $\begin{array}{l}\text { Luego de cada ciclo de pastoreo se } \\
\text { realizó una refertilización: } 100 \mathrm{~kg} / \mathrm{ha} \\
\text { de urea }\end{array}$ \\
\hline
\end{tabular}

* Por invasión de pasto blanco (Digitaria sanguinalis) se volvió a aplicar glifosato a 3,0 L/ha el 19/11/2012 y se procedió a la resiembra total el 20/11/12. Luego, el 20 y 21/11/12 se aplicó atrazina (3 L/ha) y metolaclor (1,5 L/ha). 
El sistema de pastoreo utilizado en ambos años y para ambas bases forrajeras fue rotativo, utilizando 3 parcelas de pastoreo, con 10 días de ocupación y 20 días de descanso. A la salida de los novillos de cada parcela, y en caso de considerarlo necesario, se utilizó la pasada de una rotativa para homogenizar la altura del forraje remanente de la misma.

Los animales de los tratamientos suplementados fueron racionados una vez al día, temprano en la mañana, con expeller de girasol a razón del $1 \%$ del $\mathrm{PV}$, en forma grupal (comederos). El período de acostumbramiento al suplemento fue muy reducido, ya que los animales tenían experiencia previa en el consumo de los mismos. Los animales contaron con agua de calidad y sales minerales ad libitum durante todo el período experimental.

\subsubsection{Determinaciones}

\subsubsection{Pasturas}

La disponibilidad del forraje fue determinada en las parcelas 1 y 3 de pastoreo de cada tratamiento en ambas repeticiones, a través de la realización de 5 cortes de 2 metros lineales y simultáneamente se realizaban 4 determinaciones de altura en cada línea de corte. Estas determinaciones se realizaron tanto para el forraje ofrecido como para el remanente.

Las 5 muestras cortadas (ofrecido y remanente) por parcela, se pesaban individualmente en verde y luego se juntaban en un único pool de forraje. Este se mezclaba de manera tal que cada submuestra contuviera fracciones de cada una de las muestras originales.

Para determinar el porcentaje de materia seca (MS), se utilizaba el pool de forraje restante, del que se extraían 2 submuestras. Estas se pesaban individualmente en verde para posteriormente ser secadas a estufa a $60^{\circ} \mathrm{C}$ hasta peso constante. Luego, con el peso verde de cada corte individual y el porcentaje de materia seca promedio, se estimaba la disponibilidad de forraje/ha para cada una de las muestras.

Para determinar la composición botánica, una vez pesadas en verde las 5 mues- tras de cada parcela, se tomaban 5 plantas enteras. Las mismas se separaban en las fracciones tallo (tallo + vaina), hoja (lámina) y panoja, restos secos y malezas. Estas fracciones se pesaban en verde, para luego ser secadas a $60^{\circ} \mathrm{C}$ hasta peso constante y determinar su peso seco.

El valor nutritivo del forraje se determinó con las mismas muestras utilizadas para determinar el contenido de materia seca, una vez secadas a $60^{\circ} \mathrm{C}$. Las muestras se molían y mezclaban uniformemente para posteriormente enviar una submuestra al Laboratorio de Nutrición Animal de INIA La Estanzuela. Allí se determinaron los porcentajes de las fracciones proteína cruda (PC) por el método de Kjeldhal (1984), y fibra detergente neutro (FDN) y fibra detergente ácido (FDA) por el método de Van Soest (1982). La digestibilidad de la MS (DMS) fue calculada utilizando la fórmula de Holland y Kezar (1990): DMS = 88,9 - (\%FDA x 0,779).

El valor nutritivo del suplemento se realizó a partir de una muestra representativa de cada bolsa de expeller de girasol, de $200 \mathrm{~g}$ aproximadamente, a efectos de construir un pool semanal, para su posterior evaluación en el Laboratorio de Nutrición Animal del INIA La Estanzuela. Los parámetros evaluados fueron los porcentajes de: PC, FDN y FDA. La metodología de análisis de estas fracciones fue determinada por los mismos métodos que el forraje. La fracción cenizas fue determinada mediante incineración a $300^{\circ} \mathrm{C}$ durante 3 horas.

\subsubsection{Animales}

El peso vivo lleno (PVLL) se registró al inicio, cada 14 días y al final de cada período experimental. El peso vivo vacío (PVV) se registró con 16 horas de ayuno, al inicio y al final del período experimental.

La carga parasitaria de los animales fue evaluada a través de muestras de materia fecal de todos los animales el día del inicio del ensayo y posteriormente cada 28 días, a los efectos de realizar el análisis coprológico correspondiente. Todos los animales fueron dosificados cuando el conteo de los huevos por gramo de materia fecal (HPG) superaba 500 en la mitad más uno de los animales, 
en al menos una de las parcelas. El procedimiento utilizado para estimar los HPG fue mediante la técnica de Mc Master modificada por Willamson et al. (1994).

El comportamiento animal fue evaluado en tres instancias durante el desarrollo de los ensayos. En cada ocasión, las observaciones se realizaron a lo largo de las horas luz del día, cada 15 minutos, registrándose las siguientes actividades: pastoreo, caminata, rumia, descanso (incluyó juegos, rascado, etc.), consumo de suplemento, consumo de sal y consumo de agua. Además, se tomaron mediciones de la tasa de bocado en 4 momentos del día; 2 en la mañana y 2 en la tarde. La metodología para estas determinaciones consistía en medir el tiempo requerido para efectuar 20 bocados (Jamieson y Hodgson, 1979).

La cantidad de suplemento ofrecido se ajustaba cada 14 días, de acuerdo al peso vivo promedio de los animales en cada parcela. Para estimar el consumo de suplemento, se colectó, de existir, el suplemento remanente (no consumido) en forma diaria.

Se realizaron medidas por ultrasonografía, a los efectos de medir el área del ojo de bife (AOB; entre la $12^{\circ}$ y $13^{\circ}$ costilla), cobertura de grasa (EGS; a nivel del AOB y $\mathrm{P} 8$ a nivel del cuadril) y contenido de grasa intramuscular (GIM en \%). También se realizó la medida de altura de anca en las mismas fe- chas en que se realizaron las ultrasonografías.

Una vez finalizados los ensayos, se calculó la eficiencia de conversión a través de la cantidad de suplemento consumido necesario para ganar $1 \mathrm{~kg}$ de peso animal, utilizando el consumo de suplementos en base seca.

\subsubsection{Diseño experimental}

El diseño experimental utilizado fue factorial de $2 \times 2$ completamente aleatorizado con dos repeticiones, en donde se evaluó el efecto de dos tipos de pasturas (sudangrás y sorgo BMR) y el uso de la suplementación (con y sin suplemento). Las variables fueron analizadas mediante modelos mixtos utilizando el procedimiento MIXED del paquete estadístico SAS (SAS, 2013).

\subsection{Resultados}

A continuación se presentan los resultados del ensayo, primero discriminado por el año de evaluación y finalmente se realiza un análisis conjunto de los dos años.

\subsubsection{Año 1}

\subsubsection{Resultados en pasturas}

El Cuadro 3 presenta los resultados registrados en la pastura. En el mismo puede

Cuadro 3. Efecto de la base forrajera y la suplementación de novillos sobre el forraje de sudangrás y sorgo (Año 1).

\begin{tabular}{|c|c|c|c|c|c|c|c|}
\hline \multirow{2}{*}{ Parámetro } & \multicolumn{3}{|c|}{ Pastura } & \multicolumn{3}{|c|}{ Suplemento } & \multirow{2}{*}{$\begin{array}{c}\text { P } \\
\text { Past }^{\star} \text { Sup }\end{array}$} \\
\hline & Sudan & Sorgo & $\mathbf{P}$ & No & $\mathrm{Si}$ & $\mathbf{P}$ & \\
\hline \multicolumn{8}{|c|}{ OFRECIDO } \\
\hline Altura $(\mathrm{cm})$ & 77,9 & 77,8 & ns & $71,2^{b}$ & $84,5^{a}$ & ** & ns \\
\hline $\begin{array}{l}\text { Disponibilidad } \\
\text { (kg MS/ha) }\end{array}$ & 2237,2 b & $3427,3^{a}$ & ** & $2639,1^{b}$ & $3025,4^{a}$ & * & ns \\
\hline \multicolumn{8}{|c|}{ REMANENTE } \\
\hline Altura & 40,1 & 39,9 & ns & $33,4^{b}$ & $46,6^{a}$ & ** & ns \\
\hline $\begin{array}{l}\text { Disponibilidad } \\
\text { (kg MS/ha) }\end{array}$ & $1127,7^{b}$ & $1607,4^{a}$ & ** & $1173,2^{b}$ & $1560,9^{a}$ & ** & ns \\
\hline \multicolumn{8}{|c|}{ UTILIZACIÓN } \\
\hline Utilización (\%) & $46,9^{b}$ & $53,4^{a}$ & * & $54,4^{a}$ & $45,9^{b}$ & ** & ns \\
\hline
\end{tabular}

Nota: medias con letras diferentes entre columnas son significativamente diferentes; ${ }^{*}=\mathrm{P}<0,05$; ${ }^{\star *}=\mathrm{P}<0,01$; ns= no significativo. 
observarse que la altura de la pastura del forraje ofrecido y remanente no se vio afectada por el tipo de sorgo utilizado. Sin embargo, la disponibilidad del forraje ofrecido fue significativamente superior en el caso del sorgo forrajero, coincidente con lo reportado por Kalton (1988), Miron et al. (2007), Moyer et al. (2004), citados por Silungwe (2011), quienes encontraron que los sorgos híbridos presentan una mayor productividad que el sudangrás. Rovira (2002) registró disponibilidades promedio de sudangrás a una carga de 5,3 animales/ha de 4366 y $2031 \mathrm{~kg} \mathrm{MS} / \mathrm{ha}$ para el forraje ofrecido y remanente, respectivamente. Por otro lado, en sorgo forrajero, Giorello et al. (2012) reportaron una producción superior a $10000 \mathrm{~kg} \mathrm{MS/ha}$ acumulados en el total del período evaluado.

La suplementación afectó significativamente los parámetros de la pastura, determinando mayores disponibilidades y alturas tanto del forraje ofrecido como del remanente en aquellos tratamientos suplementados, demostrando un efecto de sustitución. Este efecto se da en aquellos casos en donde el forraje es de alta calidad y la disponibilidad no es limitante, si bien se hace más relevante cuanto mayor calidad presente la pastura (Elizalde, 2003).

En cuanto a la utilización del forraje, se observa que los animales realizaron un mayor aprovechamiento en el caso del sorgo forrajero y cuando los animales no fueron suplementados. Este último resultado podría estar determinando un fenómeno de sustitu- ción del forraje por suplemento en los tratamientos suplementados. Rovira (2002), registró una utilización promedio de 53,5\% al evaluar novillos pastoreando sudangrás con una disponibilidad promedio de 4366 $\mathrm{kg} \mathrm{MS/ha} \mathrm{y} \mathrm{una} \mathrm{carga} \mathrm{de} \mathrm{5,3} \mathrm{an/ha.}$

Por otra parte, vale la pena mencionar que la interacción entre los factores evaluados no fue significativa para ninguna de las variables descritas en el Cuadro 3.

El Cuadro 4 presenta el valor nutritivo de ambas bases forrajeras, según tratamiento.

Ninguno de los parámetros referentes a la calidad de la pastura ni sus interacciones fueron afectados por los factores evaluados $(P>0,05)$, tanto para el forraje ofrecido como para el remanente. Pigurina y Methol (2004), encontraron un promedio de $7,9 \%$ de PC en una pastura de sudangrás y de 6,0 \% en sorgo. Por otra parte, Rovira y Echeverría (2014) reportaron un contenido de PC de $14,7 \%$ y $5,0 \%$ para la fracción hoja y tallo de sudangrás, respectivamente.

Esquivel et al. (2006), trabajando con novillos pastoreando sudangrás, encontraron valores de PC de 7,8 y $7,5 \%$ en el primer y segundo ciclo de pastoreo, respectivamente. Por su parte, Berlangeri (2008) reportó valores de 13,0 y $12,6 \%$ de PC en el forraje disponible de un cultivo de sorgo forrajero BMR ofrecido a novillos. En cuanto al porcentaje de FDA en el forraje ofecido, Esquivel et al. (2006) reportaron valores superiores a

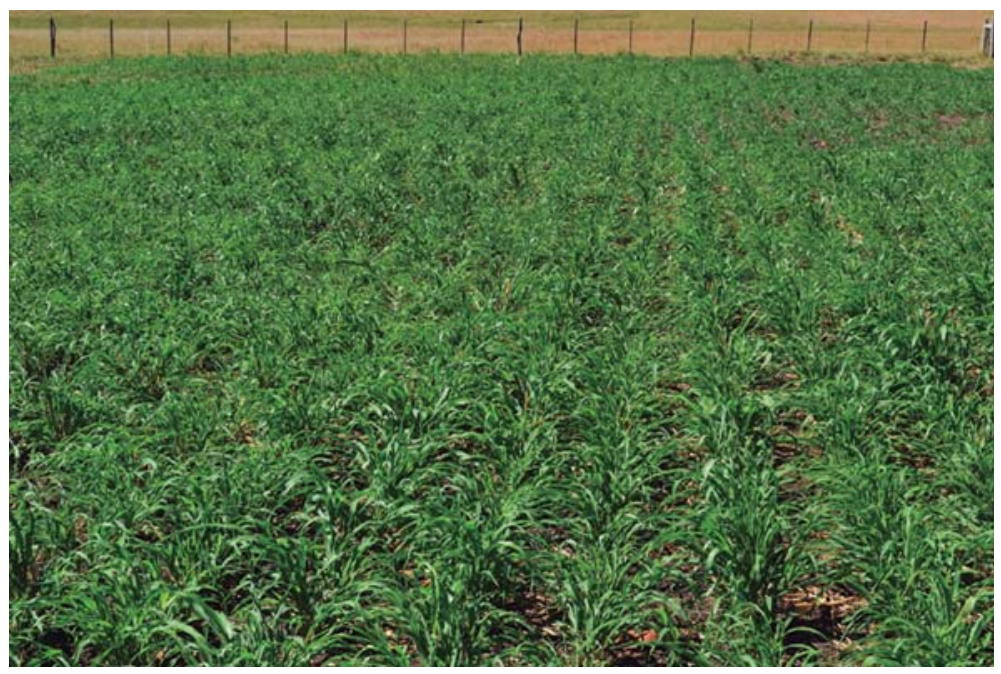


Cuadro 4. Efecto de la base forrajera y la suplementación de novillos sobre los parámetros de calidad de la pastura de sudangrás y sorgo (Año 1).

\begin{tabular}{|c|c|c|c|c|c|c|c|}
\hline \multirow{2}{*}{$\%$} & \multicolumn{3}{|c|}{ Pastura } & \multicolumn{3}{|c|}{ Suplemento } & \multirow{2}{*}{$\begin{array}{c}\text { P } \\
\text { Past }{ }^{\star} \text { Supl }\end{array}$} \\
\hline & Sudan & Sorgo & $\mathbf{P}$ & No & Si & $\mathbf{P}$ & \\
\hline \multicolumn{8}{|c|}{ OFRECIDO } \\
\hline PC & 9,0 & 9,2 & ns & 8,8 & 9,3 & ns & ns \\
\hline FDA & 37,9 & 37,8 & ns & 37,7 & 38,0 & ns & ns \\
\hline FDN & 63,6 & 62,9 & ns & 63,3 & 63,2 & ns & ns \\
\hline DMS & 59,4 & 59,5 & ns & 59,5 & 59,3 & ns & ns \\
\hline \multicolumn{8}{|c|}{ REMANENTE } \\
\hline PC & 4,7 & 4,2 & ns & 4,6 & 4,3 & ns & ns \\
\hline FDA & 46,8 & 45,0 & ns & 46,1 & 45,7 & ns & ns \\
\hline FDN & 72,9 & 70,9 & ns & 71,7 & 72,0 & ns & ns \\
\hline DMS & 52,5 & 53,9 & ns & 53,0 & 53,0 & ns & ns \\
\hline
\end{tabular}

Nota: $\mathrm{ns}=$ no significativo; $\mathrm{PC}=$ proteína cruda; FDA = fibra detergente ácido; FDN = fibra detergente neutro; DMS = Digestibilidad de la materia seca .

los encontrados en el presente trabajo $(46,6$ y $42,0 \%$ para el primer y segundo ciclo de pastoreo, respectivamente), mientras que Berlangeri (2008) halló valores de FDA que se ubicaron en 32,2 y $31,6 \%$ para dos materiales distintos de sorgo BMR.

Por otra parte los contenidos de proteína del forraje remanente se encuentran por debajo del contenido de proteína en el forraje ofrecido, en todos los escenarios evaluados. Lo inverso sucede con el contenido de FDA y FDN, lo que evidencia la selectividad de los animales, con una calidad inferior en el forraje remanente. La selectividad de los rumiantes a favor de determinados componentes de un forraje ha sido

Cuadro 5. Efecto de la base forrajera y la suplementación de novillos sobre la composición botánica promedio de la pastura de sudangrás y sorgo (Año 1).

\begin{tabular}{|c|c|c|c|c|c|c|c|}
\hline \multirow{2}{*}{$\%$} & \multicolumn{3}{|c|}{ Pastura } & \multicolumn{3}{|c|}{ Suplemento } & \multirow{2}{*}{$\begin{array}{c}\text { P } \\
\text { Past*Supl }\end{array}$} \\
\hline & Sudan & Sorgo & $\mathbf{P}$ & No & $\mathrm{Si}$ & $\mathbf{P}$ & \\
\hline \multicolumn{8}{|c|}{ OFRECIDO } \\
\hline RS & 3,0 & 2,8 & ns & 3,0 & 2,8 & ns & ns \\
\hline MS verde & 97,0 & 97,2 & ns & 97,0 & 97,2 & ns & ns \\
\hline Hoja ${ }^{a}$ & 26,6 & 29,4 & ns & 29,0 & 27.0 & ns & ns \\
\hline Tallo $^{\mathrm{a}}$ & 71,1 & 69,2 & ns & 69,2 & 71,1 & ns & ns \\
\hline Panoja $^{a}$ & 2,3 & 1,4 & ns & 1,9 & 1,8 & ns & ns \\
\hline \multicolumn{8}{|c|}{ REMANENTE } \\
\hline RS & 1,6 & 1,3 & ns & 1,5 & 1,3 & ns & ns \\
\hline MS verde & 98,4 & 98,7 & ns & 98,5 & 98,7 & ns & ns \\
\hline Hoja ${ }^{a}$ & 9,0 & 18,6 & ns & 12,4 & 15,2 & ns & ns \\
\hline Tallo ${ }^{\mathrm{a}}$ & 90,9 & 81,4 & ns & 87,6 & 84,7 & ns & ns \\
\hline Panoja ${ }^{a}$ & 0,1 & 0,0 & ns & 0,0 & 0,1 & ns & ns \\
\hline
\end{tabular}

Nota: ns = no significativo; MS = contenido de materia seca; $\mathrm{RS}$ = fracción restos secos. ${ }^{\text {a }}$ respecto a la materia seca verde. 
ampliamente documentada (Chacon y Stobbs, 1976; Van Dyne, 1980; Arnold, 1981; Clark et al., 1982; Hodgson, 1982, 1985, 1990; L'Hullier et al., 1984; Vallentine, 1990; citados por Montossi et al., 2000).

El Cuadro 5 presenta la composición botánica promedio de todo el período de cada base forrajera, según el tratamiento aplicado.

Al igual que lo observado para los parámetros de valor nutritivo del forraje, ni los factores tipo de forraje y suplementación, ni la interacción entre estos afectaron significativamente ninguno de los componentes de la composición botánica $(P>0,05)$. Tomando en cuenta los resultados en disponibilidad, valor nutritivo y composición botánica, se puede decir que solamente la cantidad del forraje fue afectada por los tratamientos aplicados, mientras que la calidad del mismo no fue afectada por estos.

En un trabajo experimental realizado por Rovira y Echeverría (2014), se reportaron

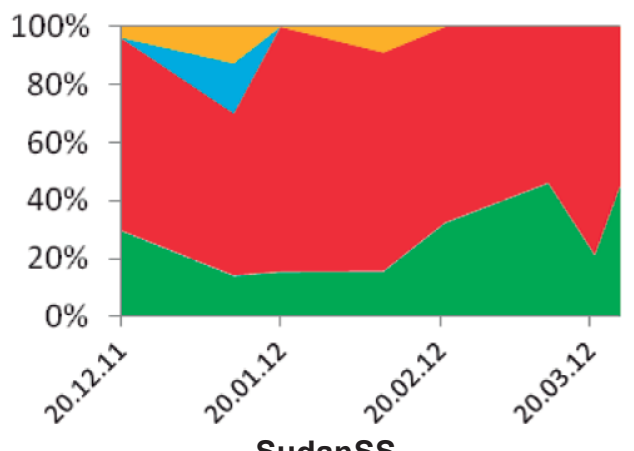

Sudanss

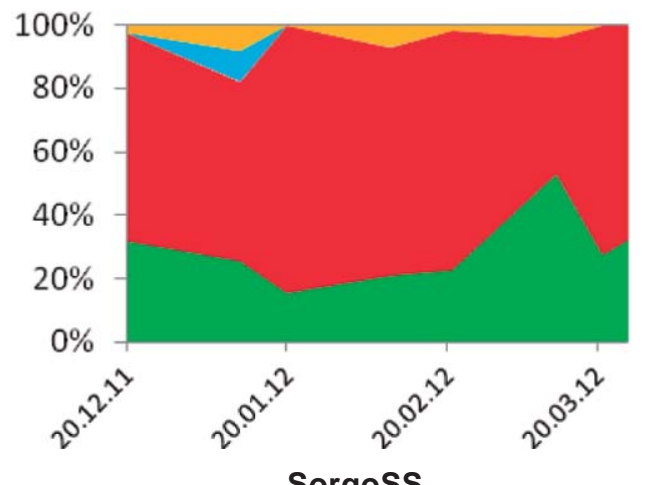

Sorgoss porcentajes de hoja de $48 \%$ en sudangrás y $49 \%$ en sorgo BMR en el primer pastoreo, $25 \%$ en sudangrás y $26 \%$ en sorgo BMR en el segundo ciclo de pastoreo y $24 \%$ en sudangrás y $21 \%$ en sorgo BMR en el tercer ciclo de pastoreo, en el forraje ofrecido. Los porcentajes de hojas reportados por estos autores son sensiblemente mayores a los obtenidos en el presente ensayo. Las diferencias entre ambas experiencias podrían estar asociadas al tipo de cultivar utilizado, la estrategia de manejo del pastoreo, suplementación diferencial, etc.

Se destaca la muy baja proporción de panojas y restos secos para ambas bases forrajeras, lo cual sería un indicador del correcto manejo del pastoreo durante el ensayo, ya que no se le permitió llegar a estadios reproductivos.

La Figura 1 presenta la evolución en el tiempo de la composición botánica del forraje ofrecido de cada base forrajera, se-

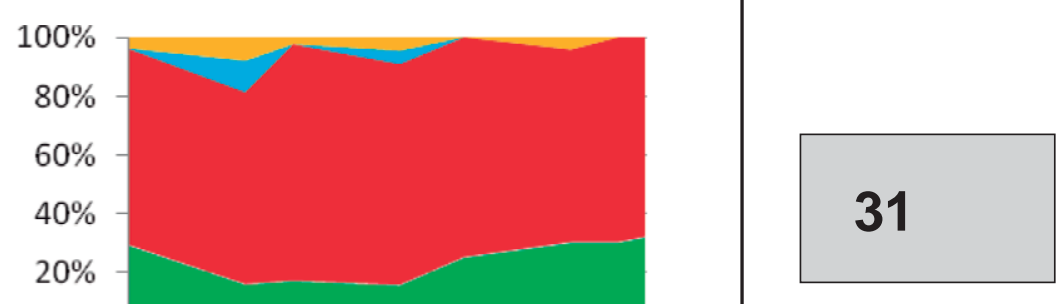

Nota: $S S=$ sin suplementación; CS = con suplementación; $H=$ hoja; $T$ = tallo; $P$ = panoja; $R S=$ restos secos.

Figura 1. Evolución de la composición botánica del forraje ofrecido de sudangrás y sorgo forrajero, con y sin suplementación, bajo pastoreo de novillos (Año 1). 
gún los cuatro tratamientos resultantes de la interacción de los factores, sudangrás sin suplemento (SudanSS), sudangrás con suplemento (SudanCS), sorgo sin suplemento (SorgoSS) y sorgo con suplemento (SorgoCS).

La fracción tallo dominó la composición del forraje en todos los tratamientos, a lo largo de todo el período experimental. La proporción de hojas, especialmente en el cultivo de sorgo, tuvo un aumento hacia el final del período, lo cual se vio reflejado en la calidad del forraje disponible al final del período experimental.

La Figura 2 presenta la evolución de la composición botánica del forraje remanente en cada tratamiento.

La composición botánica del sudangrás se comportó de manera similar entre los tratamientos sin suplementación y con suplementación, con predominio neto de la fracción tallo a lo largo de todo el período (Figura 2). Algo muy similar se registró en el sor-
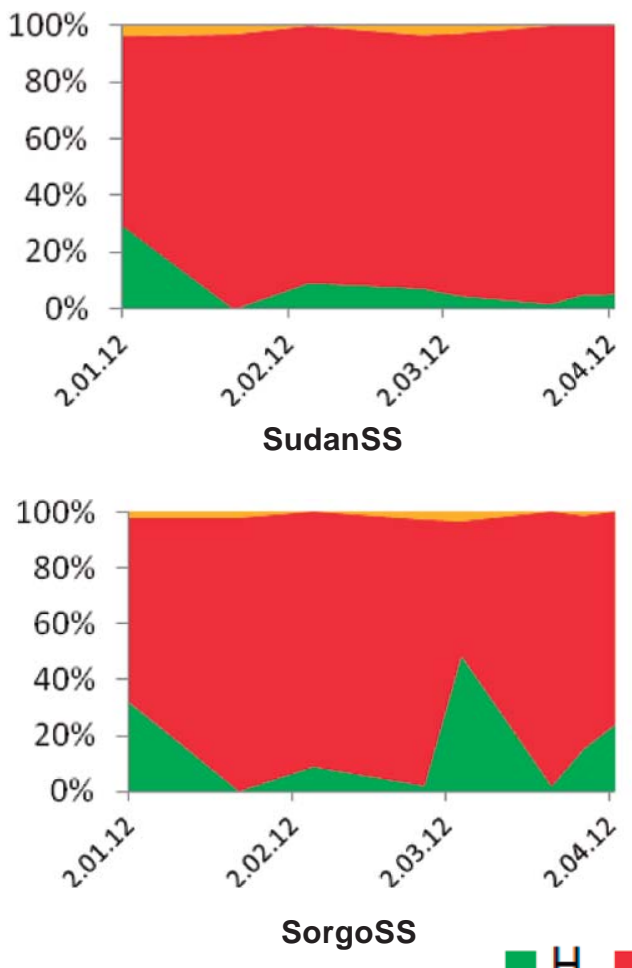

Sorgoss go forrajero. Sin embargo, el sorgo tuvo oscilaciones más marcadas en la proporción de tallo y hoja.

\subsubsection{Resultados en comportamiento y producción animal}

Se presentan primero los resultados registrados en el comportamiento de pastoreo de los animales, el cual estaría asociado a los resultados del desempeño animal.

El Cuadro 6 muestra los resultados obtenidos en el comportamiento animal a partir de los dos factores evaluados (tipo de forraje y uso de suplemento) y en la Figura 3 se presentan los resultados del comportamiento animal y tasa de bocado promedio, para cada uno de los tratamientos evaluados.

El tipo de forraje utilizado no tuvo efecto en ninguno de los parámetros estudiados ni en la tasa de bocado. Sin embargo, el uso de suplemento determinó diferencias significativas $(P<0,05)$ en algunas de las activi-
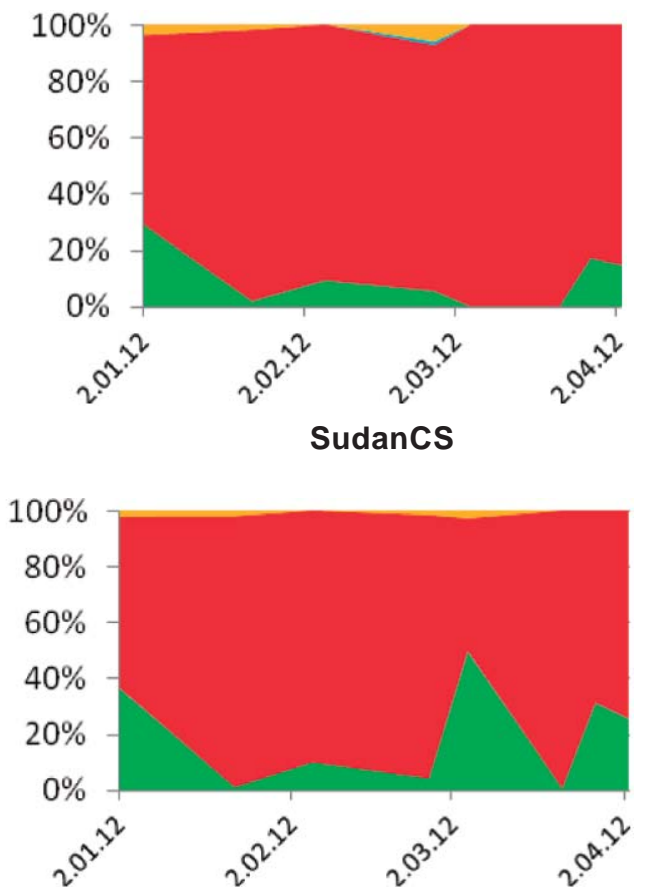

SorgoCS

Nota: $\mathrm{SS}=$ sin suplementación; $\mathrm{CS}$ = con suplementación; $\mathrm{H}=$ hoja; $\mathrm{T}$ = tallo; $\mathrm{P}$ = panoja; $\mathrm{RS}=$ restos secos.

Figura 2. Evolución de la composición botánica del forraje remanente de sudangrás y sorgo forrajero, con y sin suplementación, bajo pastoreo de novillos (Año1). 
Cuadro 6. Efecto de la base forrajera y la suplementación de novillos sobre la conducta animal (Año 1).

\begin{tabular}{|c|c|c|c|c|c|c|c|}
\hline \multirow{2}{*}{$\%$} & \multicolumn{3}{|c|}{ Pastura } & \multicolumn{3}{|c|}{ Suplemento } & \multirow{2}{*}{$\begin{array}{c}\text { P } \\
\text { Past }^{\star} \text { Supl }\end{array}$} \\
\hline & Sudan & Sorgo & $\mathbf{P}$ & No & Si & $\mathbf{P}$ & \\
\hline Pastoreo & 43,1 & 41,7 & ns & $48,9^{a}$ & $35,9^{b}$ & ** & ns \\
\hline Rumia & 14,7 & 16,0 & ns & $18,8^{a}$ & $12,0^{b}$ & ** & ns \\
\hline Suplemento & 2,9 & 2,2 & ns & $0,0^{b}$ & $5,1^{a}$ & ** & ns \\
\hline Agua & 3,9 & 4,9 & ns & 3,7 & 5,1 & ns & $\star \star$ \\
\hline Caminar & 1,7 & 1,6 & ns & 1,8 & 1,5 & ns & ns \\
\hline Descanso & 33,7 & 33,6 & ns & $26,9^{b}$ & $40,4^{a}$ & ** & ns \\
\hline $\begin{array}{l}\text { Tasa de bocado } \\
\text { (bocado/minuto) }\end{array}$ & 11,6 & 11,7 & ns & $12,8^{a}$ & $10,5^{b}$ & ** & ns \\
\hline
\end{tabular}

Nota: medias con letras diferentes entre columnas son significativamente diferentes; ns = no significativo; ${ }^{*}=\mathrm{P}<0,05 ;{ }^{*}=\mathrm{P}<0,01$. Nota: Las actividades realizadas por los animales se expresan como porcentaje del tiempo total.

dades comportamentales. Como era de esperar, el tiempo dedicado al pastoreo fue mayor en los animales que no recibieron expeller de girasol en su dieta, determinando un mayor tiempo dedicado a la rumia y un menor tiempo de descanso. En la tasa de bocado también se observa una diferencia significativa $(P<0,05)$, siendo mayor la cantidad de bocados por minuto en los animales no suplementados. Esto podría explicarse porque el incremento en la tasa de bocado es una respuesta compensatoria que tiene el animal para prevenir un descenso en la tasa de consumo total (Penning et al., 1991, citados por Montossi, 1995).

La interacción de los factores presentó diferencias significativas $(P<0,05)$ únicamente en el tiempo destinado al consumo de agua, siendo en los animales que se encontraron pastoreando sorgo y expeller de girasol mayor $(7,0 \%)$ que en los restantes tratamientos $(4,3 \%$ Sudanss, 3,2 \%

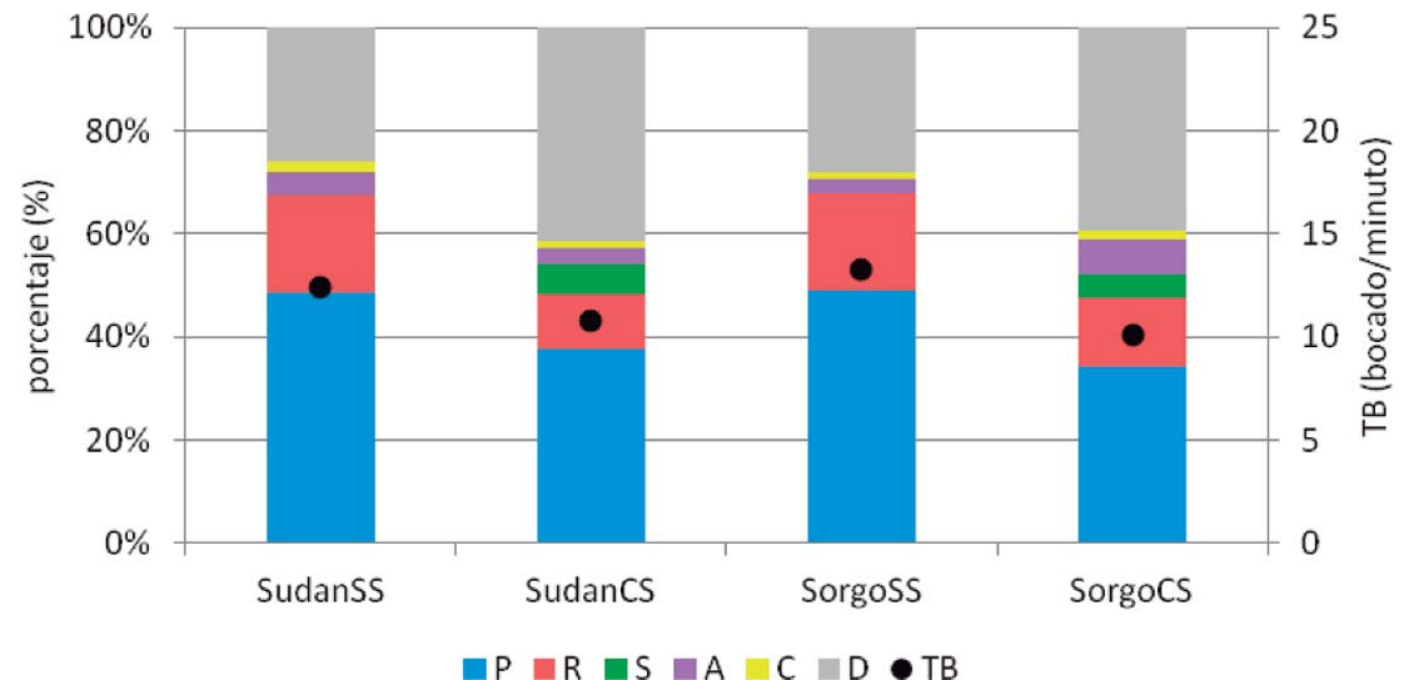

Nota: $\mathrm{SS}=$ sin suplementación; $\mathrm{CS}$ = con suplementación; $\mathrm{TB}$ = tasa de bocado; $\mathrm{P}$ = actividad de pastoreo; $\mathrm{R}$ = actividad de rumia; $\mathrm{S}=$ actividad de consumo de suplemento; $\mathrm{A}=$ actividad de consumo de agua; $\mathrm{C}=$ actividad de caminata; $\mathrm{D}=$ descanso.

Figura 3. Efecto de la base forrajera y la suplementación sobre el comportamiento animal y tasa de bocado de novillos sobre verdeo estival (Año1). 


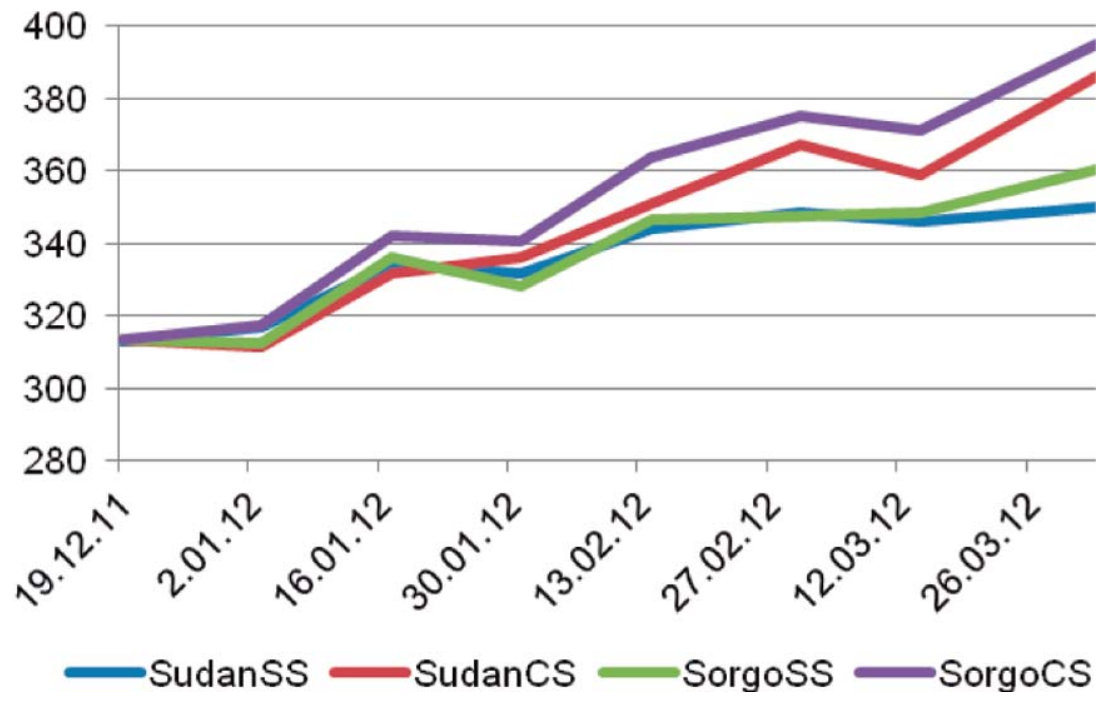

Figura 4. Evolución del peso vivo de novillos sobre verdeos de verano (Año 1).

SudanCS y $2,8 \%$ SorgoSS) que no presentaron diferencias entre ellos.

La Figura 4 presenta la evolución del PV de cada tratamiento. En el Cuadro 7 se observan los principales resultados obtenidos en relación al desempeño animal para cada factor.

Al inicio del estudio los animales no presentaron diferencias en el peso promedio en cada uno de los tratamientos evaluados. Los pesos vivos llenos finales no presentaron diferencias significativas $(P>$
$0,05)$ cuando fueron analizados según el forraje utilizado. Sin embargo, para el factor suplementación, sí se encontraron diferencias estadísticas a favor de los animales que recibieron expeller de girasol durante el período de estudio. Las ganancias medias diarias llenas, presentaron diferencias para ambos factores estudiados, siendo superiores las de los animales pastoreando sorgo y los que fueron suplementados. Para las ganancias medias de peso vivo vacío se observaron valores

Cuadro 7. Efecto de la base forrajera y la suplementación sobre la performance animal de novillos sobre verdeo estival (Año 1).

\begin{tabular}{|c|c|c|c|c|c|c|c|}
\hline \multirow{2}{*}{ Parámetro } & \multicolumn{3}{|c|}{ Pastura } & \multicolumn{3}{|c|}{ Suplemento } & \multirow{2}{*}{$\begin{array}{c}\text { P } \\
\text { Past*Supl }\end{array}$} \\
\hline & Sudan & Sorgo & $\mathbf{P}$ & No & Si & $\mathbf{P}$ & \\
\hline PVLL i (kg) & 313,3 & 313,4 & ns & 313,1 & 313,6 & ns & ns \\
\hline PVLL $\mathrm{f}(\mathrm{kg})$ & 368,2 & 377,7 & ns & $355,4^{b}$ & $390,5^{a}$ & $\star *$ & * \\
\hline PVV i (kg) & 286,8 & 287,3 & ns & 289,6 & 284,4 & ns & ns \\
\hline$P \vee \vee f(k g)$ & 354,1 & 359,9 & ns & $345,0^{b}$ & $369,0^{a}$ & * & ns \\
\hline AA i (cm) & 123,7 & 124,1 & ns & 123,4 & 124,4 & ns & ns \\
\hline$A A f(c m)$ & 127,6 & 128,1 & ns & 127,5 & 128,2 & ns & ns \\
\hline GPVLL (kg/an/día) & $0,522^{b}$ & $0,613^{a}$ & $\star \star$ & $0,403^{b}$ & $0,732^{a}$ & $\star \star$ & ns \\
\hline GPVV (kg/an/día) & 0,641 & 0,691 & ns & $0,528^{b}$ & $0,805^{a}$ & ** & ns \\
\hline Producción (kg PV/ha) & $411,4^{b}$ & $482,6^{a}$ & $\star *$ & $317,3^{b}$ & $576,8^{a}$ & $\star *$ & ns \\
\hline
\end{tabular}

Nota: medias con letras diferentes entre columnas son significativamente diferentes; ns = no significativo; ${ }^{*}=\mathrm{P}<0,05 ;{ }^{*}=\mathrm{P}<0,01 ; \mathrm{i}=$ inicial $; \mathrm{f}=$ final; $\mathrm{PVLL}=$ peso vivo lleno; $\mathrm{PVV}=$ peso vivo vacío; $\mathrm{AA}=$ altura de anca; GPV = ganancia de peso vivo. 
mayores $(P<0,05)$ únicamente en los animales suplementados.

Vaz Martins et al. (2003) registraron ganancias en un rango de 0,718-0,880 kg/an/día, obtenidas en 101-118 días de pastoreo en total, en novillos pastoreando sudangrás sin suministro de suplemento. Rovira y Echeverría (2013) presentaron ganancias promedio en 73 días de ensayo sobre la misma base forrajera de 0,823 kg/an/día. Estas ganancias fueron obtenidas a una dotación que varió entre 4,5 y 7,0 novillos/ha.

Por otro lado, Rovira (2002) registró ganancias de 0,451 kg/an/día durante dos ciclos de pastoreo en novillos sobre sudangrás, utilizando una carga de 5,3 novillos/ha, durante un período de 53 días. Esquivel et al. (2006) observaron ganancias de 0,473 $\mathrm{kg} / \mathrm{an} / \mathrm{d}$ ía con novillos pastoreando sudangrás a una carga de 7,0 animales/ha y durante dos ciclos de pastoreo de 68 días en total.

En experiencias sobre sorgo forrajero sin suplementación, Vaz Martins et al. (2003), lograron ganancias en un rango de 0,788 $0,980 \mathrm{~kg} / \mathrm{an} / \mathrm{día}$ con períodos experimentales entre 79 y 107 días y Rovira y Echeverria (2013), durante un período de 73 días obtuvieron ganancias de 0,945 kg/an/día.

La interacción de los dos factores de estudio presentó diferencias significativas únicamente en el peso vivo lleno final, en donde los mayores pesos $(P<0,05)$ correspondieron a los animales suplementados, seguidos por el sorgo sin suplementar y por último el sudangrás sin agregado de expeller en la dieta (SorgoCS 394,8 kg, SudanCS 386,1 kg, SorgoSS 360,6 kg, SudanSS 350,2 kg).

Como ya se mencionó previamente, los valores promedio de valor nutricional y composición botánica no fueron estadísticamente diferentes $(P>0,05)$ en los factores evaluados. Por lo tanto, los resultados en producción animal pueden ser explicados por la cantidad de forraje, es decir, por la asignación forrajera y también por la presencia o ausencia de la suplementación. Los animales que pastorearon sorgo forrajero tenían significativamente más disponibilidad de forraje ofrecido que aquellos que pastorearon sudangrás. Asimismo, el contenido de materia seca del forraje de sudangrás fue, en términos generales, mayor que en el sorgo. Todo esto podría explicar por qué se evidencia una tendencia a un menor desempeño animal en el tratamiento sudangrás sin suplemento y una tendencia a un mejor desempeño sobre sorgo que sobre sudangrás en los tratamientos suplementados.

Las eficiencias de conversión (EC) no difirieron estadísticamente $(P>0,05)$ entre los tratamientos con inclusión de suplementación, siendo de 10,3 kg de suplemento/kg PV ( $\mathrm{kg} \mathrm{S} / \mathrm{kg} \mathrm{PV}$ ) en animales pastoreando sudangrás (SudanCS) y de $11,0 \mathrm{~kg} \mathrm{~S} / \mathrm{kg}$ PV en sorgo (SorgoCS). Simeone y Beretta (2004) presentaron eficiencias de conversión de novillos Hereford (280 kg PV) pastoreando praderas mezclas de gramíneas y leguminosas durante el verano de 6:1, 9:1 y 45:1, suplementados con grano de maíz al $1 \%$ PV, para asignaciones de forraje de 3,6 y $9 \%$, respectivamente.

Rovira (2002), evaluando novillos pastoreando sudangrás sin suplementación, encontró que la ganancia diaria animal estuvo correlacionada positivamente con el porcentaje de hojas disponible en el cultivo. En el presente trabajo, la proporción de hojas en el forraje entre tratamientos no difirió estadísticamente, tanto para forraje disponible como remanente. No obstante, al observar la evolución de la composición botánica en el tiempo, el tratamiento SorgoCS presenta una proporción de hojas en el forraje ofrecido mayor hacia el final del ensayo y esta fracción del forraje aumentó más drásticamente desde la mitad del período experimental en adelante. Al observar la gráfica de evolución del PV (Figura 4), puede notarse que en el tratamiento SorgoCS, comienza a diferenciarse en forma positiva del resto de los tratamientos a mitad del período evaluado. Si se observa el desempeño de los animales de los tratamientos CS, se puede señalar que a partir de mediados de enero, la ganancia de peso vivo resulta mayor que en los tratamientos no suplementados y esta tendencia continúa hasta que se hace notoriamente evidente a partir del inicio del mes de marzo.

En el Cuadro 8 se presentan los resultados de producción animal relacionados a la calidad de canal in vivo. 
Cuadro 8. Efecto de la base forrajera y la suplementación sobre la calidad de canal y carne de novillos sobre verdeo estival (Año 1)

\begin{tabular}{|c|c|c|c|c|c|c|c|}
\hline \multirow{2}{*}{ Parámetro } & \multicolumn{3}{|c|}{ Pastura } & \multicolumn{3}{|c|}{ Suplemento } & \multirow{2}{*}{$\begin{array}{c}\text { P } \\
\text { Past*Supl }\end{array}$} \\
\hline & Sudan & Sorgo & $\mathbf{P}$ & No & Si & $\mathbf{P}$ & \\
\hline AOB i $\left(\mathrm{cm}^{2}\right)$ & 29,4 & 30,2 & ns & 30,2 & 29,5 & ns & ns \\
\hline AOB $f\left(\mathrm{~cm}^{2}\right)$ & 48,2 & 48,8 & ns & 46,9 & 50,1 & ns & ns \\
\hline EGS i (mm) & 2,1 & 2,1 & ns & 2,0 & 2,1 & ns & ns \\
\hline EGS $f(m m)$ & 3,1 & 3,4 & ns & $2,9^{b}$ & $3,6^{a}$ & * & ns \\
\hline P8 i (mm) & 2,0 & 2,2 & ns & 1,9 & 2,2 & ns & ns \\
\hline P8 f (mm) & 3,8 & 4,4 & ns & $3,5^{b}$ & $4,8^{a}$ & ** & ns \\
\hline GIM i (\%) & 2,0 & 2,2 & ns & 2,3 & 1,9 & ns & ns \\
\hline GIM f (\%) & 3,0 & 3,1 & ns & 3,1 & 3,0 & ns & ns \\
\hline
\end{tabular}

Nota: medias con letras diferentes entre columnas son significativamente diferentes; ns = no significativo; ${ }^{*}=\mathrm{P}<0,05 ;{ }^{* *}=\mathrm{P}<0,01 ; \mathrm{i}=$ inicial; $\mathrm{f}=$ final; $\mathrm{AOB}=$ área ojo de bife; $\mathrm{EGS}=$ espesor grasa subcutánea; P8 = espesor de grasa del cuadril; GIM = grasa intramuscular.

Las medidas registradas de área de ojo de bife (AOB), espesor de grasa subcutánea (EGS), espesor de grasa a nivel del cuadril (P8) y contenido de grasa intramuscular (GIM), al inicio del experimento no presentaron diferencias significativas en los diferentes factores evaluados. Al final del estudio, AOB y GIM fueron estadísticamente iguales en las diferentes alternativas nutricionales.

Al final del experimento, el efecto de la suplementación solo fue estadísticamente significativo $(P<0,05)$ para el espesor de grasa subcutánea (EGS) y a nivel del cuadril (P8), lográndose mayores valores en los animales suplementados. Los valores promedio, teniendo en cuenta únicamente el factor tipo de pastura, no difirieron estadísticamente $(P>0,05)$ en estos dos parámetros evaluados.

En ninguno de los parámetros de calidad de la canal y carne analizados se observaron efectos de la interacción entre el tipo de forraje y el uso de suplemento.

\subsubsection{Comentarios y conclusiones del Año 1}

Las características de cantidad del forraje ofrecido de los cultivos, más que las características de calidad de los mismos, fueron las determinantes en afectar el desempeño animal, así como la inclusión o no de la suplementación. Si bien el forraje disponi- ble fue significativamente mayor en el caso del sorgo forrajero, estas diferencias no fueron reflejadas de la misma manera en las variables referentes al desempeño animal. De hecho, más allá de la tendencia a favor del sorgo en comparación con el sudangrás, el factor más relevante en cuanto al desempeño animal individual y por unidad de superficie, fue la inclusión de la suplementación.

Esto concuerda con los resultados de comportamiento animal, en donde nuevamente se destaca que la inclusión de la suplementación tuvo un efecto en las proporciones de tiempo asignados a cada actividad por parte de los animales. Los animales que mejor desempeño lograron fueron los suplementados, que destinaron menos tiempo al pastoreo en proporción a las demás actividades. Cosgrove y Edwards (2007) señalan que las pasturas son ecosistemas complejos con múltiples interacciones que ocurren entre los componentes de estas, y entre el ganado y las plantas. En este sentido, la actividad de pastoreo implicaría costos energéticos asociados a la cosecha del forraje (Nicol y Brookes, 2007), lo cual, en este caso determinaría un gasto de energía mayor en los animales que destinaron mayor tiempo a esta actividad.

En términos generales y desde el punto de vista productivo, los tratamientos suplementados resultaron significativamente su- 
periores que los no suplementados (Cuadro 7). De la misma forma, los tratamientos sobre sudangrás presentaron valores algo inferiores en comparación con el sorgo forrajero, si bien desde el punto de vista estadístico estas diferencias no fueron significativas para todos los parámetros evaluados. Por otra parte, la inclusión de un suplemento sí tiene un impacto de mayor relevancia, por lo cual, a pesar de haberse demostrado la conveniencia productiva de suplementar los animales, esta decisión por parte del productor deberá estar basada -contando con los coeficientes generados en este y otros experimentos- en la relación costo/beneficio de esta práctica.

\subsubsection{Año 2}

\subsubsection{Resultados en pasturas}

El Cuadro 9 presenta los resultados promedio registrados en la pastura durante el período de estudio.

Como fue mencionado en la sección 2.1, se realizaron cortes de pastura para determinar el forraje ofrecido y remanente. Durante este año, el último corte correspondiente al final del ensayo no pudo realizarse por exceso de precipitaciones. Para poder realizar una mejor comprension de la evolución del forraje ofrecido y del remanente, se presenta por un lado la disponibilidad promedio total del período y por otro la disponibilidad promedio de los cortes realizados para ofrecido y remanente, que corresponden con los cortes de estos últimos. La utilización del forraje fue calculada teniendo en cuenta los cortes de disponible con sus respectivos cortes de remanente.

En los parámetros estudiados, el tipo de pastura determinó diferencias significativas $(P<0,05)$ en la disponibilidad de forraje ofrecido y remanente, sin presentar efecto en la altura del forraje. El sorgo forrajero presentó mayores niveles de disponibilidad de forraje ofrecido. Las mayores disponibilidades del sorgo forrajero en comparación al sudangrás son coincidentes con lo señalado por Silungwe (2011). Por otra parte, la suplementación no afectó ninguna de las variables de la pastura analizadas.

La utilización del forraje calculada para el período enero-febrero no difirió $(P>0,05)$ entre las dos bases forrajeras, ni tampoco

Cuadro 9. Efecto de la base forrajera y la suplementación de novillos sobre el forraje de sudangrás y sorgo (Año 2).

\begin{tabular}{|c|c|c|c|c|c|c|c|}
\hline \multirow{2}{*}{ Parámetro } & \multicolumn{3}{|c|}{ Pastura } & \multicolumn{3}{|c|}{ Suplemento } & \multirow{2}{*}{$\begin{array}{c}\text { P } \\
\text { Past*Supl }\end{array}$} \\
\hline & Sudan & Sorgo & $\mathbf{P}$ & No & Si & $\mathbf{P}$ & \\
\hline \multicolumn{8}{|c|}{ OFRECIDO } \\
\hline Altura (cm) & 93,6 & 98,8 & ns & 95,5 & 96,9 & ns & ns \\
\hline Altura $(\mathrm{cm})^{1}$ & 137,9 & 143,7 & ns & 141,1 & 140,6 & ns & ns \\
\hline $\begin{array}{l}\text { Disponible } \\
\text { (kg MS/ha) }\end{array}$ & $2700,5^{b}$ & $4107,4^{a}$ & ** & 3502,8 & 3305,1 & ns & ns \\
\hline $\begin{array}{l}\text { Disponible } \\
(\mathrm{kg} \mathrm{MS} / \mathrm{ha})^{1}\end{array}$ & $3295,4^{b}$ & $5507,5^{a}$ & ** & 4570,0 & 4232,9 & ns & ns \\
\hline \multicolumn{8}{|c|}{ REMANENTE } \\
\hline Altura $(\mathrm{cm})^{1}$ & 93,3 & 104,8 & ns & 93,7 & 104,4 & ns & ns \\
\hline $\begin{array}{l}\text { Disponible } \\
(\mathrm{kg} \mathrm{MS} / \mathrm{ha})^{1}\end{array}$ & $1777,1^{\mathrm{b}}$ & $2686,7^{a}$ & ** & 2383,5 & 2080,4 & ns & ns \\
\hline \multicolumn{8}{|c|}{ UTILIZACIÓN } \\
\hline Utilización (\%) & 45,5 & 49,2 & ns & 48,0 & 46,7 & ns & ns \\
\hline
\end{tabular}

Nota: medias con letras diferentes entre columnas son significativamente diferentes; ns = no significativo; $* *=\mathrm{P}<0,01 ; \mathrm{MS}=$ contenido de materia seca. ${ }^{1}$ durante el período enero - febrero. 
entre los animales sin suplementación y los suplementados.

En este segundo año de evaluación, las utilizaciones fueron menores que en el primer año, pero en este caso los tratamientos no tuvieron un efecto significativo $(P>0,05)$ en la utilización de la pastura. Rovira (2002), logró valores de utilización promedio superiores $(53,5 \%)$ a los encontrados en este ensayo pastoreando un sudangrás con una disponibilidad de $4366 \mathrm{~kg} \mathrm{MS} / \mathrm{ha}$, sin suplementación. Sin embargo, existen registros reportados por Esquivel et al. (2006) con utilizaciones de sudangrás menores de 36 y $45 \%$ para el primer y segundo pastoreo, con disponibilidades de 6084 y $4309 \mathrm{~kg} \mathrm{MS} / \mathrm{ha}$, respectivamente. Las utilizaciones registradas para sorgo son comparables a lo registrado por Vaz Martins et al. (2003) quienes lograron valores de 45-53\% en un cultivo con disponibilidades entre 1331-1549 kg MS/ha, sin suplementación.

En el Cuadro 10 se presentan los resultados del valor nutritivo de cada verdeo, según el factor evaluado.

Al igual que en el Año 1, el valor nutricional del forraje ofrecido no difirió entre los materiales forrajeros utilizados y el uso o no de la suplementación. En el forraje remanen- te, los contenidos de proteína cruda fueron similares para ambos factores estudiados. Sin embargo, los contenidos de FDA y FDN, y la digestibilidad de la materia seca fueron superiores $(P<0,05)$ en el sudangrás en comparación con el sorgo, determinando una mejor calidad del forraje remanente para el caso del sorgo.

En el Cuadro 11 se presenta la composición botánica promedio de cada base forrajera utilizada y el efecto del uso de la suplementación.

Al igual que en el primer año de evaluación, la composición botánica y contenido de materia seca del forraje ofrecido no fueron afectados significativamente $(P>0,05)$ por el tipo de forraje utilizado o por la inclusión del suplemento en el sistema de engorde. Comparando los valores del forraje ofrecido entre años se constata que estos fueron muy similares, a excepción del contenido de la fracción panoja.

Al analizar el forraje remanente, se encontraron algunas diferencias en la composición botánica en el caso del factor suplementación, siendo superior la proporción de tallo y menor la de panoja $(\mathrm{P}<0,05)$ en las parcelas en donde pastorearon los animales que no fueron suplementados.

Cuadro 10. Efecto de la base forrajera y la suplementación de novillos sobre la calidad de la pastura (Año 2).

\begin{tabular}{|c|c|c|c|c|c|c|c|}
\hline \multirow{2}{*}{$\%$} & \multicolumn{3}{|c|}{ Pastura } & \multicolumn{3}{|c|}{ Suplemento } & \multirow{2}{*}{$\begin{array}{c}\text { P } \\
\text { Past*Supl }\end{array}$} \\
\hline & Sudan & Sorgo & $\mathbf{P}$ & No & Si & $\mathbf{P}$ & \\
\hline \multicolumn{8}{|c|}{ OFRECIDO } \\
\hline PC & 9,0 & 11,0 & ns & 10,3 & 9,6 & ns & ns \\
\hline FDA & 37,0 & 34,9 & ns & 35,3 & 36,6 & ns & ns \\
\hline FDN & 62,8 & 61,7 & ns & 61,6 & 63,0 & ns & ns \\
\hline DMS & 60,1 & 61,7 & ns & 61,4 & 60,3 & ns & ns \\
\hline \multicolumn{8}{|c|}{ REMANENTE } \\
\hline PC & 4,0 & 4,3 & ns & 4,4 & 3,9 & ns & ns \\
\hline FDA & $41,6^{a}$ & $34,7^{b}$ & ** & 37,8 & 38,5 & ns & ns \\
\hline FDN & $65,7^{a}$ & $56,6^{b}$ & $\star \star$ & 60,7 & 61,7 & ns & ns \\
\hline DMS & $56,5^{b}$ & $61,9^{a}$ & ** & 59,5 & 58,9 & ns & ns \\
\hline
\end{tabular}

Nota: medias con letras diferentes entre columnas son significativamente diferentes; ns = no significativo; ** $=\mathrm{P}<0,01 ; \mathrm{PC}=$ proteína cruda; FDA = fibra detergente ácido; FDN = fibra detergente neutro; DMS = Digestibilidad de la materia seca. 
Cuadro 11. Efecto de la base forrajera y la suplementación de novillos sobre la composición botánica de la pastura de sudangrás y sorgo (Año 2).

\begin{tabular}{|c|c|c|c|c|c|c|c|}
\hline \multirow{2}{*}{$\%$} & \multicolumn{3}{|c|}{ Pastura } & \multicolumn{3}{|c|}{ Suplemento } & \multirow{2}{*}{$\begin{array}{c}\text { P } \\
\text { Past*Supl }\end{array}$} \\
\hline & Sudan & Sorgo & $\mathbf{P}$ & No & Si & $\mathbf{P}$ & \\
\hline \multicolumn{8}{|c|}{ OFRECIDO } \\
\hline $\mathrm{RS}$ & 3,5 & 2,2 & ns & 3,3 & 2,4 & ns & ns \\
\hline MS verde & 96,5 & 97,8 & ns & 96,7 & 97,6 & ns & ns \\
\hline $\mathrm{Hoja}^{1}$ & 29,9 & 34,2 & ns & 30,2 & 33,8 & ns & ns \\
\hline Tallo $^{1}$ & 70,1 & 63,1 & ns & 69,0 & 64,2 & ns & ns \\
\hline Panoja $^{1}$ & 0,0 & 2,7 & ns & 0,7 & 2,0 & ns & ns \\
\hline \multicolumn{8}{|c|}{ REMANENTE } \\
\hline $\mathrm{RS}$ & 2,0 & 1,7 & ns & 1,7 & 2,0 & ns & ns \\
\hline MS verde & 98,0 & 98,3 & ns & 98,3 & 98,0 & ns & ns \\
\hline Hoja ${ }^{1}$ & 9,9 & 9,0 & ns & 7,9 & 11,0 & ns & ns \\
\hline Tallo ${ }^{1}$ & 87,2 & 85,3 & ns & $91,5^{\text {a }}$ & $81,0^{b}$ & ** & ns \\
\hline Panoja $^{1}$ & 3,0 & 5,6 & ns & $0,6^{b}$ & $8,0^{a}$ & * & ns \\
\hline
\end{tabular}

Nota: medias con letras diferentes entre columnas son significativamente diferentes; ns = no significativo; ${ }^{*}=\mathrm{P}<0,05 ;{ }^{*}=\mathrm{P}<0,01 ; \mathrm{MS}=$ contenido de materia seca; $\mathrm{RS}=$ fracción restos secos. ${ }^{1}$ en relación al contenido de MS verde.

Cabe destacar que no se registraron interacciones significativas $(P>0,05)$ entre los factores evaluados para ninguna de las fracciones de la composición botánica.

La Figura 5 presenta la evolución (en porcentaje) de la composición botánica de forraje ofrecido y de la interacción entre los dos factores evaluados.
En el cultivo de sudangrás, el contenido de hojas fue mayor al principio y tuvo un aumento hacia el final del período. Al comparar la representación gráfica de la Figura 5 con los del Año 1 (Figura 1), se encuentra que en ambos años se registró el mismo aumento de esta fracción hacia el útlimo tercio del período experiemental. En este segundo año de evaluación, el contenido de hojas del fo-

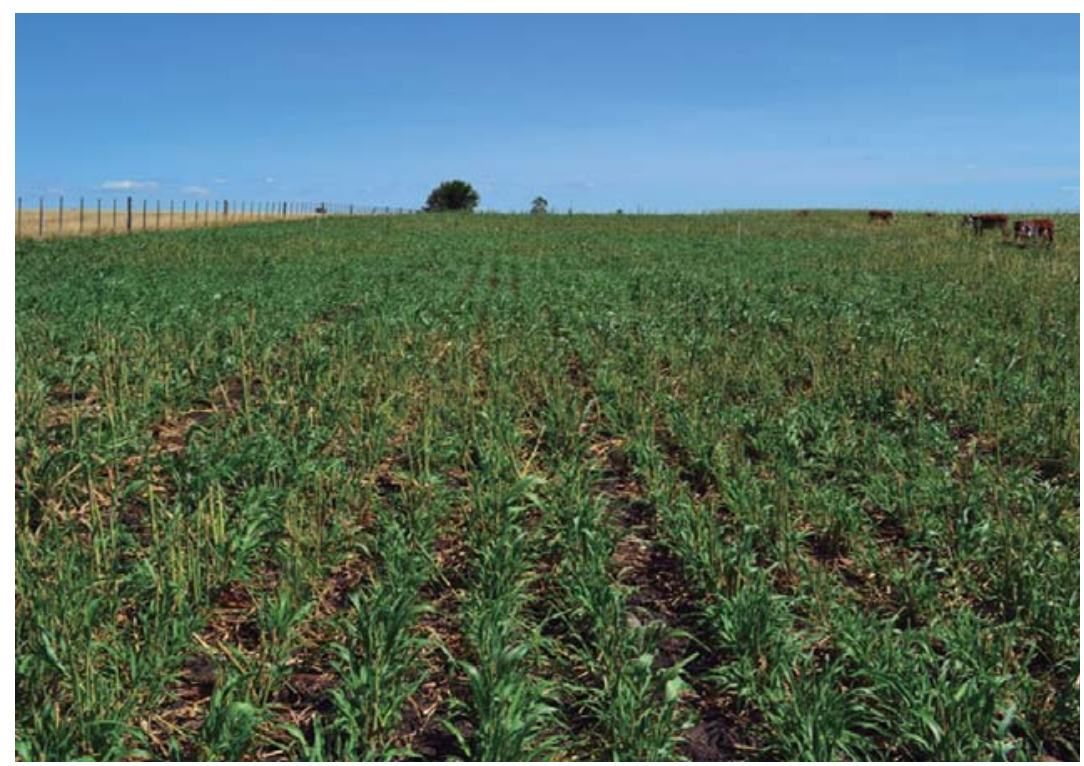




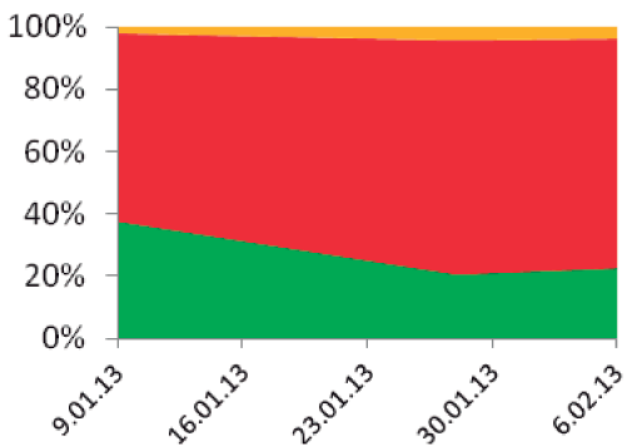

Sudanss

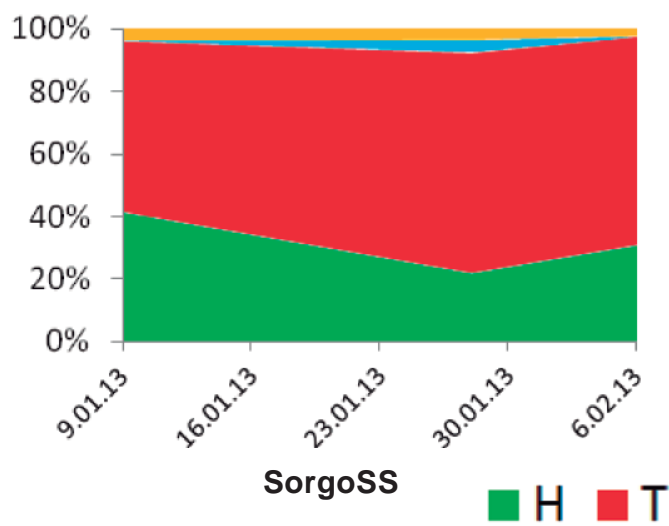

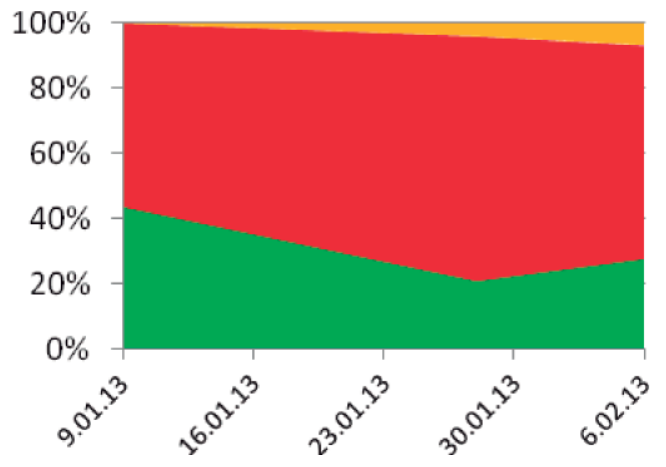

SudanCS

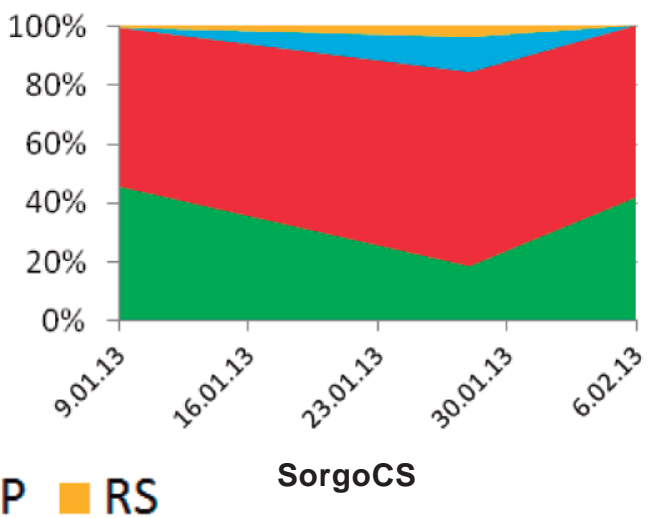

Nota: $\mathrm{SS}=$ sin suplementación; $\mathrm{CS}=$ con suplementación; $\mathrm{H}=$ hoja; $\mathrm{T}=$ tallo; $\mathrm{P}=$ panoja; $\mathrm{RS}=$ restos secos.

Figura 5. Evolución de la composición botánica del forraje ofrecido de sudangrás y sorgo forrajero, con y sin suplementación, bajo pastoreo de novillos (Año 2).

rraje ofrecido en el tratamiento Sudan con suplementación (SudanCS) fue siempre superior al sin suplemento (SS).

La evolución de la composición botánica del cultivo de sorgo forrajero es relativamente similar entre los tratamientos SS y CS. Sin embargo, la proporción de tallo se mantuvo relativamente constante en el tiempo para el caso de CS, mientras que para los SS aumentó este componente hacia el final del período experimental. Al contrastar los dos años de evaluación, se puede mencionar que la fracción panoja se registró más tempranamente en el Año 1 con respecto al Año 2.

La Figura 6 presenta la evolución de la composición botánica del forraje remanente para cada base forrajera, según tratamiento.

La evolución de la composición botánica del forraje remanente tuvo comportamientos muy similares entre los tratamien- tos y con muy poca variación al comparar los valores iniciales con los finales de cada fracción. En todos los casos la fracción hoja fue disminuyendo gradualmente a medida que fue transcurriendo el período de evaluación, observándose una mayor proporción de hojas en SudanCs y Sorgoss.

\subsubsection{Resultados en comportamiento y producción animal}

Al igual que para el Año 1, se presentan en esta sección los resultados registrados en comportamiento animal y posteriormente en desempeño animal.

Los resultados obtenidos en el comportamiento animal a partir de los dos factores evaluados (tipo de forraje y uso de suplemento) se muestran en el Cuadro 12, mientras que la Figura 7 presenta los resultados de comportamiento animal. 

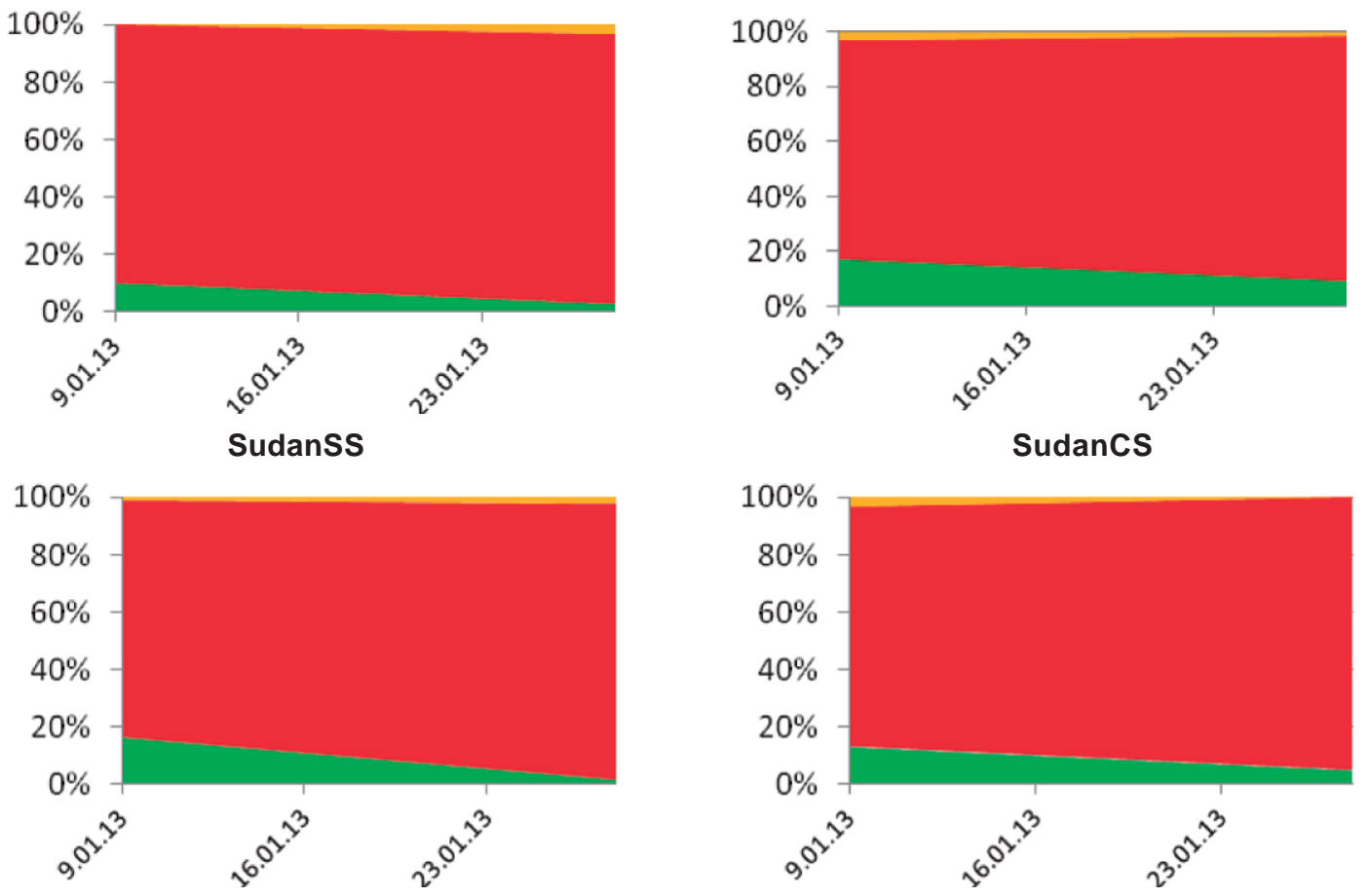

Sorgoss

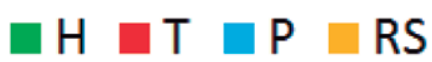

SorgoCs

Nota: $\mathrm{SS}=$ sin suplementación; $\mathrm{CS}=$ con suplementación; $\mathrm{H}=$ hoja; $\mathrm{T}$ = tallo; $\mathrm{P}$ = panoja; $\mathrm{RS}=$ restos secos.

Figura 6. Evolución de la composición botánica del forraje remanente de sudangrás y sorgo forrajero, con y sin suplementación, bajo pastoreo de novillos (Año 2).

Cuadro 12. Efecto de la base forrajera y la suplementación de novillos sobre la conducta animal (Año 2).

\begin{tabular}{|c|c|c|c|c|c|c|c|}
\hline \multirow{2}{*}{$\%$} & \multicolumn{3}{|c|}{ Pastura } & \multicolumn{3}{|c|}{ Suplemento } & \multirow{2}{*}{$\begin{array}{c}\text { P } \\
\text { Past*Supl }\end{array}$} \\
\hline & Sudan & Sorgo & $\mathbf{P}$ & No & Si & $\mathbf{P}$ & \\
\hline \multicolumn{8}{|c|}{ OFRECIDO } \\
\hline Pastoreo & 52,2 & 53,3 & ns & $62,8^{a}$ & $42,7^{b}$ & ** & ns \\
\hline Rumia & $9,8^{b}$ & $14,3^{a}$ & * & $12,5^{a}$ & $9,7^{\mathrm{b}}$ & * & ns \\
\hline Suplemento & $5,0^{a}$ & $3,7^{b}$ & * & - & 8,7 & - & * \\
\hline Agua & $2,7^{b}$ & $4,3^{a}$ & ** & $2,5^{b}$ & $4,6^{a}$ & ** & ns \\
\hline Caminar & 1,5 & 2,2 & ns & 2,0 & 1,7 & ns & ns \\
\hline Descanso & 27,8 & 23,3 & ns & $19,6^{b}$ & $31,4^{a}$ & ** & ns \\
\hline $\begin{array}{l}\text { Tasa de bocado } \\
\text { (bocados/minuto) }\end{array}$ & 15,4 & 14,7 & ns & 15,9 & 14,2 & ns & ns \\
\hline
\end{tabular}

Nota: medias con letras diferentes entre columnas son significativamente diferentes; ns = no significativo; ${ }^{*}=\mathrm{P}<0,05 ;{ }^{*}=\mathrm{P}<0,01$.

No se registraron diferencias significativas en la actividad de pastoreo al comparar las dos bases forrajeras. Sin embargo, al evaluar el efecto de la suplementación, se observa que aquellos animales sin acceso al suplemento dedicaron significativamente mayor tiempo $(P<0,05)$ al pastoreo y a la rumia. Esto podría explicarse por el hecho de que los animales no suplementados debieron cubrir sus requeri- 


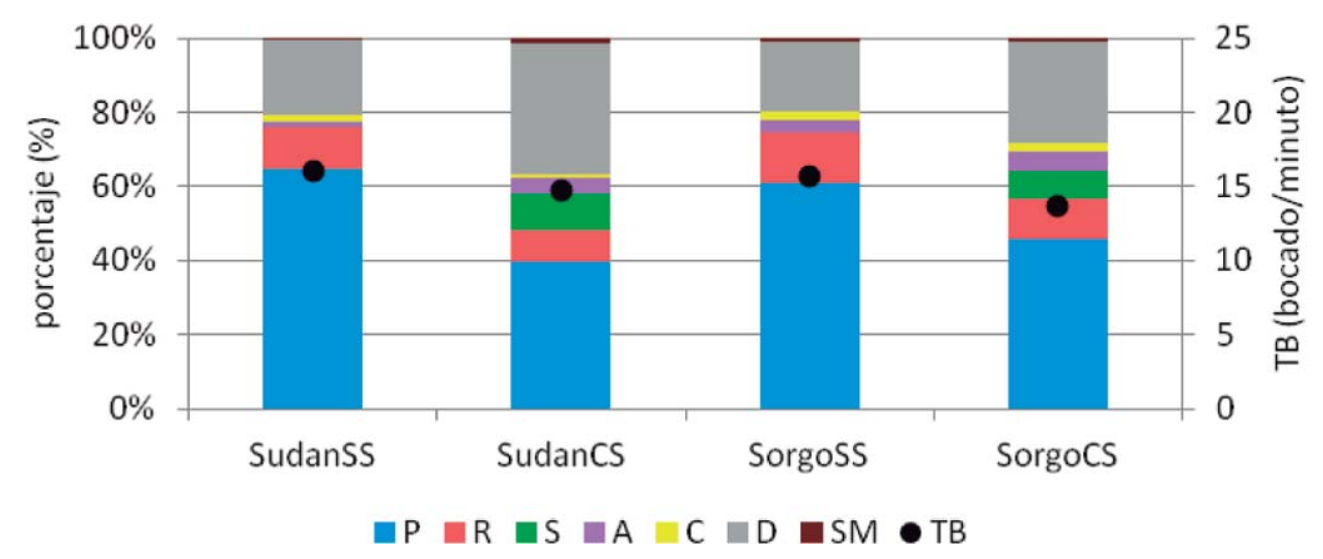

Nota: $\mathrm{SS}$ = sin suplementación; $\mathrm{CS}$ = con suplementación; $\mathrm{TB}$ = tasa de bocado; $\mathrm{P}$ = actividad de pastoreo; $\mathrm{R}$ = actividad de rumia; $S$ = actividad de consumo de suplemento; $A=$ actividad de consumo de agua; $C=$ actividad de caminata; $\mathrm{D}=$ descanso; $\mathrm{SM}=$ consumo de sal mineral

Figura 7. Efecto de la base forrajera y la suplementación sobre el comportamiento animal y la tasa de bocado de novillos (Año 2).

mientos dedicando un mayor tiempo al pastoreo que sus contrapartes suplementados (Montossi, 1995).

En cuanto al tiempo dedicado al consumo de suplemento, los animales pastoreando sorgo dedicaron un menor tiempo $(\mathrm{P}<$ $0,05)$ a esta actividad en comparación a aquellos que pastorearon sudangrás (3,7 vs. $5,0 \%)$.

En este segundo año de evaluación no se detectaron diferencias significativas $(P>$ $0,05)$ en las tasas de bocado según la hora del día, ni en los valores promedio. Esto contrasta con lo hallado en el Año 1, donde se encontraron diferencias en la tasa de bocado entre los animales suplementados y no suplementados, donde fueron menores para los animales suplementados.
La interacción entre los factores del animal y del forraje influye en la selectividad y el valor nutricional de la dieta, las cuales actúan conjuntamente determinando la tasa de bocado y el consumo total del animal (Gordon, 2000). Según Wade y Carvalho (2000), la altura del forraje sería un factor determinante en el consumo diario de forraje. Se debe tener en cuenta que el pastoreo es una actividad que resulta de interacciones muy complejas entre las plantas y los animales (Cosgrove y Edwards, 2007), por lo tanto, no puede simplificarse todo a uno o dos factores en el momento de explicar el comportamiento animal, en este caso la tasa de bocado, sin considerar el tamaño del bocado y el valor nutricional de la dieta.

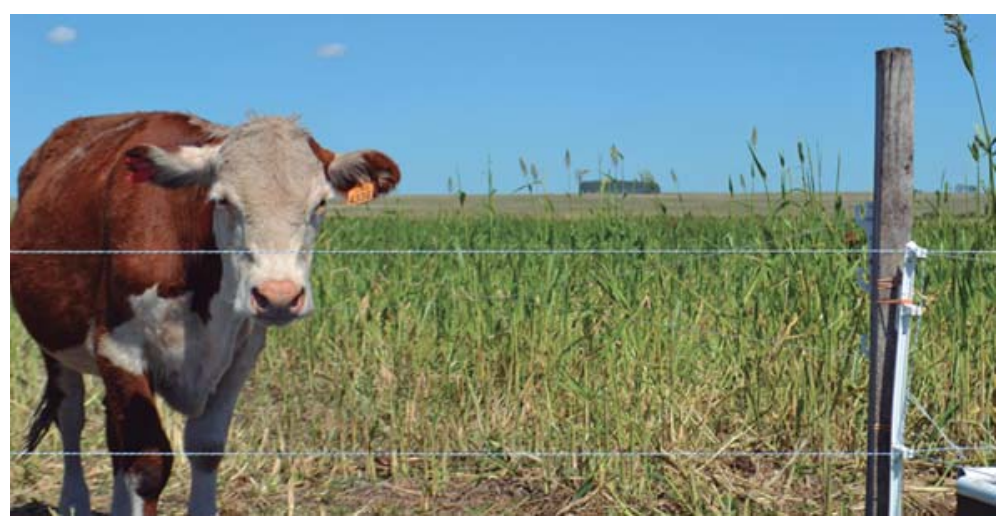


En el Cuadro 13 se presentan los principales resultados obtenidos en desempeño animal, mientras que en la Figura 8 se presenta la evolución del peso vivo de los novillos de cada tratamiento.

Durante el segundo año de estudio, las diferencias encontradas en los pesos vivos Ilenos finales se debieron principalmente al efecto de la suplementación, siendo mayores $(P<0,05)$ en los animales que consumieron expeller de girasol. Asimismo, a pe- sar de no haber encontrado efecto del tipo de pastura, la interacción entre los dos factores estudiados (forraje y uso de suplemento) mostró diferencias $(P<0,05)$ en los resultados finales, siendo el Sorgo suplementado (SorgoCS) $(362,2 \mathrm{~kg}$ ) estadísticamente superior a Sudan sin suplementación (SudanSS) $(339,5 \mathrm{~kg})$ y ubicándose los restantes tratamientos en posiciones intermedias (356,9 kg SudanCS y 346,7 kg SorgoSS).

Cuadro 13. Efecto de la base forrajera y la suplementación sobre el desempeño animal de novillos (Año 2).

\begin{tabular}{|c|c|c|c|c|c|c|c|}
\hline \multirow{2}{*}{ Parámetro } & \multicolumn{3}{|c|}{ Pastura } & \multicolumn{3}{|c|}{ Suplemento } & \multirow{2}{*}{$\begin{array}{c}\text { P } \\
\text { Past*Supl }\end{array}$} \\
\hline & Sudan & Sorgo & $\mathbf{P}$ & No & Si & $\mathbf{P}$ & \\
\hline PVLL i (kg) & 295,6 & 295,5 & ns & 295,5 & 295,5 & ns & ns \\
\hline PVLL f (kg) & 348,2 & 354,5 & ns & $343,1^{b}$ & $359,6^{a}$ & $\star \star$ & * \\
\hline PVV i (kg) & 271,0 & 270,3 & ns & 263,3 & 271,9 & ns & ns \\
\hline PVV f (kg) & 330,4 & 334,4 & ns & $324,4^{b}$ & $340,4^{a}$ & * & * \\
\hline GPVLL (kg/an/día) & $0,908^{b}$ & $1,017^{a}$ & * & $0,821^{b}$ & $1,104^{a}$ & ** & ns \\
\hline GPVV (kg/an/día) & 1,024 & 1,106 & ns & $0,951^{b}$ & $1,179^{a}$ & ** & ns \\
\hline Producción (kg PV/ha) & $394,9^{b}$ & $442,5^{a}$ & * & $357,0^{b}$ & $480,4^{a}$ & $\star \star$ & ns \\
\hline
\end{tabular}

Nota: medias con letras diferentes entre columnas son significativamente diferentes; $n s$ = no significativo; ${ }^{*}=\mathrm{P}<0,05 ;{ }^{*}=\mathrm{P}<0.01 ; \mathrm{i}=$ inicial $; \mathrm{f}=$ final; $\mathrm{PVLL}=$ peso vivo lleno; $\mathrm{PVV}=$ peso vivo vacío; $\mathrm{AA}=$ altura de anca; GPV = ganancia de peso vivo.

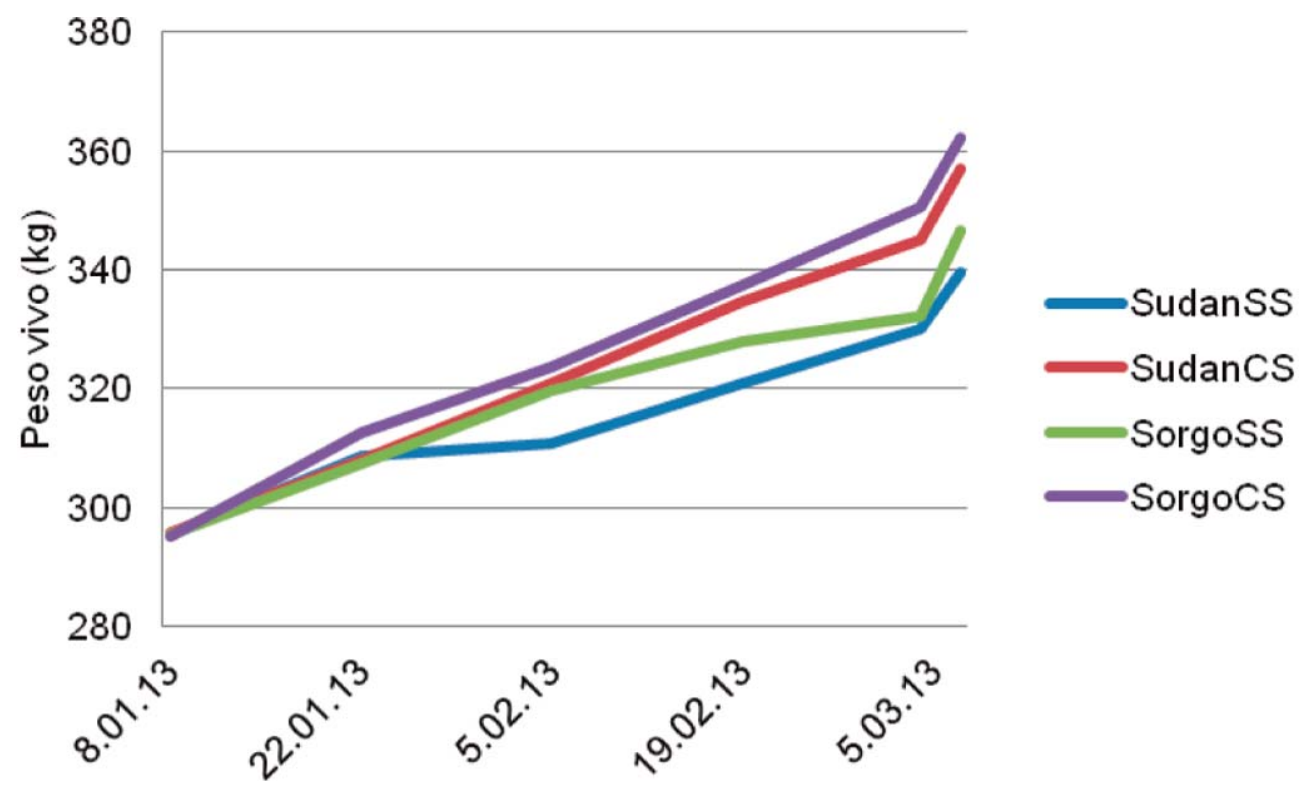

Nota: SS = sin suplementación; CS = con suplementación.

Figura 8. Evolución del peso vivo lleno de novillos sobre verdeos de verano (Año 2). 
Al observar la Figura 8 se destaca que la evolución de pesos correpondientes a los tratamientos suplementados y la correspondiente al tratamiento SorgoSS fueron muy similares hasta el principio de febrero, a partir de donde el SudanSS tiende a presentar ganancias muy inferiores con respecto a los tratamientos suplementados o al SorgoSS. Podría decirse que hasta esa fecha, la mayor productividad del sorgo que reporta la bilbiografía (Carámbula, 2007a) y que fuera mencionada previamente, se asemeja al tratamiento sobre SudanCS, mientras que el tratamiento de SorgoCS probablemente presente un fenómeno de sustitución simple o adición más sustitución de suplemento por forraje.

Hacia el final del ensayo, los tratamientos CS tienden a comportarse de manera muy similar, resaltando la importancia del factor suplementación sobre la base forrajera para explicar el mejor desempeño animal.

En el otro extremo, se encuentra la evolución del tratamiento SudanSS, que como ya se mencionó, contó con una menor cantidad de forraje disponible y esto se reflejó en la curva de crecimiento de los animales de este tratamiento en contraste con los otros tres tratamientos, al menos hasta el segundo tercio de febrero (Figura 8).

El peso vivo vacío final presentó el mismo comportamiento que el peso vivo lleno, en donde el principal efecto se asoció al suministro de suplemento con la misma respues- ta $(P<0,05)$ para la interacción entre el tipo de forraje utilizado y la inclusión de expeller de girasol en la dieta (SorgoCS $\geq$ SudanCS = SorgoSS $\geq$ SudanSS).

Las ganancias medias diarias, comparadas con el Año 1, fueron muy superiores para los dos factores evaluados por separado, sin encontrar diferencias en la interacción de los mismos $(P>0,05)$. Asimismo, en términos generales se puede decir que estos desempeños superaron todos los registros revisados de ensayos nacionales (Rovira, 2002; Vaz Martins et al., 2003; Esquivel et al., 2006; Rovira y Echeverría, 2013).

En cuanto a la producción animal por unidad de superficie, nuevamente se ubica a los animales que pastorearon sorgo y a los suplementados en las posiciones superiores.

La eficiencia de conversión del Año 2 presentó diferencias significativas $(P<0,05)$ a favor del sudangrás $(11,1 \mathrm{~kg} \mathrm{~S} / \mathrm{kg} P V)$ en relación al sorgo forrajero (12,1 kg S/kg PV).

Los resultados obtenidos del estudio realizado en las canales medidas por ultrasonografía según el efecto del forraje y la suplementación se presentan en el Cuadro 14.

Los valores de área de ojo de bife (AOB) finales fueron inferiores a los registrados en el Año 1, incluso partiendo de valores iniciales más altos. El efecto de la suplementación determinó mayores AOB $(P<0,05)$ en los animales suplementados en comparación con los que no lo fueron. Los valores finales

Cuadro 14. Efecto de la base forrajera y la suplementación sobre la calidad de canal y carne de novillos (Año 2).

\begin{tabular}{|c|c|c|c|c|c|c|c|}
\hline \multirow{2}{*}{ Parámetro } & \multicolumn{3}{|c|}{ Pastura } & \multicolumn{3}{|c|}{ Suplemento } & \multirow{2}{*}{$\begin{array}{c}\text { P } \\
\text { Past*Supl }\end{array}$} \\
\hline & Sudan & Sorgo & $\mathbf{P}$ & No & Si & $\mathbf{P}$ & \\
\hline AOB i $\left(\mathrm{cm}^{2}\right)$ & 41,7 & 40,4 & ns & 41,0 & 41,1 & ns & ns \\
\hline AOB $\mathrm{f}\left(\mathrm{cm}^{2}\right)$ & 45,3 & 46,2 & ns & $44,1^{b}$ & $47,4^{a}$ & * & ns \\
\hline EGS i (mm) & 2,1 & 2,1 & ns & 2,1 & 2,1 & ns & ns \\
\hline$E G S f(m m)$ & 2,4 & 2,5 & ns & 2,3 & 2,6 & ns & ns \\
\hline P8 i (mm) & 2,4 & 2,2 & ns & 2,3 & 2,4 & ns & ns \\
\hline$P 8 \mathrm{f}(\mathrm{mm})$ & 3,3 & 3,3 & ns & $2,9^{b}$ & $3,6^{a}$ & $\star *$ & ns \\
\hline GIM f (\%) & 2,8 & 2,7 & ns & 2,7 & 2,8 & ns & ns \\
\hline
\end{tabular}

Nota: medias con letras diferentes entre columnas son significativamente diferentes; $\mathrm{ns}=$ no significativo; ${ }^{*} \mathrm{P}<0,05 ;{ }^{*}=\mathrm{P}<0,01 ; \mathrm{i}=$ inicial; $\mathrm{f}=$ final; $\mathrm{AOB}=$ área ojo de bife; $\mathrm{EGS}=$ espesor grasa subcutánea; P8 = espesor de grasa del cuadril; GIM = grasa intramuscular. 
de espesor de grasa subcutánea (EGS) se encuentran por debajo de lo obtenido en el primer año de evaluación.

Es de destacar que no exisitieron diferencias significativas $(P>0,05)$ en la interacción entre el forraje y el suplemento para ninguna de las variables bajo estudio.

\subsubsection{Comentarios y conclusiones del Año 2}

Una vez más, las características de cantidad de forraje fueron las de mayor relevancia para explicar el desempeño animal, mientras que el valor nutricional fue menos relevante.

El factor suplementación fue determinante en los resultados finales de producción animal en general, así como en el comportamiento animal.

Al igual que en el Año 1, los tratamientos sobre sorgo forrajero tendieron a tener un desempeño animal superior en comparación a aquellos sobre sudangrás, en términos de ganancia de peso y peso vivo final (Fernández Mayer et al., 2011). En algunos casos, no se detectaron diferencias significativas en las variables del valor nutricional del forraje evaluado, pero se observan tendencias a favor del sorgo en términos de valores más altos en contenido de proteína y menores en el contenido de fibra y mayores proporciones de la fracción hoja. En otros casos, como con la disponibilidad de forraje, las diferencias sí fueron estadísticamente significativas a favor del sorgo.

Cuando se suplementa sobre un forraje de alta calidad y en condiciones no limitantes de disponibilidad, el consumo de forraje disminuye en mayor proporción que el aumento del consumo total de materia seca provocado por la suplementación. En otras palabras, se da un fenómeno de sustitución de forraje por suplemento. Normalmente los efectos de la sustitución son mayores cuando mayor es la calidad del forraje y si este no es limitante. En estos casos el animal dejará de consumir forraje y las respuestas al suplemento serán un reflejo de la calidad del suplemento en relación a la calidad del forraje (Elizalde, 2003). En tal sentido, los tratamientos con inclusión de suplementa- ción determinaron mejores desempeños animales, con mayor ganancia de peso vivo y producción por unidad de superficie que los no suplementados, al igual que en el Año 1.

\subsubsection{Comentarios y conclusiones de ambos años $(1+2)$ en conjunto}

La cantidad de forraje fue determinante en los resultados en producción animal en ambos años, que resultaron muy contrastantes en la productividad forrajera. En este contexto, la tendencia de superioridad del sorgo sobre el sudangrás se ubicó en el rango de 12 a $17 \%$ cuando estas diferencias se llevan a producción de PV/ha. Es posible obtener productividades por unidad de superficie sin suplementación entre 300 y $350 \mathrm{~kg} \mathrm{PV/ha.} \mathrm{Incluso} \mathrm{aunque}$ se pudieran lograr ganancias diarias superiores a $1,500 \mathrm{~kg} / \mathrm{an} / \mathrm{d} i ́ a$, con animales que comienzan el engorde sobre los diferentes tipos de sorgo con aproximadamente $300 \mathrm{~kg} \mathrm{PV}$, no es posible terminarlos para faena.

Con el uso de 7,5 novillos/ha sobre esta base forrajera es posible lograr ganancias diarias entre 0,400 a 0,800 kg/an/día. Por efecto de la suplementación estas aumentan a un rango de 0,700 a 1,100 kg/an/día con eficiencias de conversión en el rango de 10 a $12 \mathrm{~kg}$ de suplemento por kg PV. La suplementación permitió aumentar la productividad por unidad de superficie en el rango de 480 a $580 \mathrm{~kg} \mathrm{PV/ha,} \mathrm{pero} \mathrm{aún}$ no es posible terminar los novillos con los pesos de faena que en la actualidad requiere el mercado, cuando estos comienzan el uso de estos verdeos con $300 \mathrm{~kg}$ PV.

La suplementación tuvo la repercusión más evidente en términos de producción total, más que en performance individual. Por ende, se puede reflexionar que: i) estas opciones forrajeras permitirían ganancias individuales para acelerar el proceso de recría de novillos durante el verano, y que podrían lograrse pesos de terminación más cercanos a los adecuados para la faena, si se acelera la ganancia de peso previamente durante el proceso de recría y 
ii) que la suplementación proteica con expeller de girasol, sobre sudangrás o sorgo forrajero es una estrategia tecnológica para mejorar la productividad de esta base forrajera de manera de aumentar la productividad de los predios ganaderos del norte del Uruguay. Cabe señalar que se generaron coeficientes biológicos que permiten a productores y técnicos la evaluación económica del uso de esta tecnología para diferentes condiciones productivas y económicas.

\section{EVALUACIÓN DE NOVILLOS SOBRE SORGO FORRAJERO CON DISTINTAS CARGAS Y TIPOS DE SUPLEMENTACIÓN}

En esta sección, se presentan tres ensayos distintos centrados en: i) la evaluación del uso de distintos suplementos en novillos pastoreando sorgo, ii) la evaluación del uso de distintos suplementos combinados con distintas cargas animales de novillos y finalmente iii) la evaluación del uso de distintos tipos de suplementos en la fisiología ruminal de novillos fistulados.

\subsection{Evaluación del uso de diferentes suplementos sobre sorgo forrajero}

El primer ensayo tuvo por objetivo principal evaluar el pastoreo de un sorgo forrajero en combinación con el uso de diferentes tipos de suplemento como alternativas para acelerar el proceso de recría y/o engorde y calidad de canal de novillos durante el período estival en la región de Basalto. Este ensayo fue replicado por dos años (2014 y 2015).

Los objetivos específicos fueron:

- Evaluar el desempeño de novillos Hereford sobre un verdeo de sorgo forrajero a una carga única de 7,5 novillos/ha.

- Evaluar el efecto de la suplementación con grano de maíz (GM) al 0,5\% del peso vivo (PV) en la productividad animal (carne), sobre un verdeo de sorgo forrajero.
- Evaluar el efecto de la suplementación con afrechillo de arroz (AA) al 0,5\% del $\mathrm{PV}$ en la productividad animal (carne), sobre un verdeo de sorgo forrajero.

- Evaluar el efecto de la suplementación con expeller de girasol (EG) al 0,5\% del $\mathrm{PV}$ en la productividad animal, sobre un verdeo de sorgo forrajero.

- Evaluar el efecto de la suplementación con expeller de soja (ES) (solo año 2015) al 0,5\% del PV en la productividad animal, sobre un verdeo de sorgo forrajero.

- Evaluar el efecto de la suplementación en la productividad de un verdeo de sorgo forrajero.

- Evaluar el efecto de los tratamientos sobre la deposición de tejidos en el animal in vivo.

\subsubsection{Materiales y métodos}

Este ensayo se realizó durante dos años. El primer año, el período experimental comenzó el 11 de diciembre de 2013 y finalizó el 27 de marzo de 2014 (106 días), mientras que el segundo año, el período experimental comenzó el 18 de diciembre de 2014 y finalizó el 25 de marzo de 2015 (96 días).

Los novillos se encontraban a una única carga de 7,5 novillos/ha. Se evaluaron 4 tratamientos en el año 1 (2014) y 5 tratamientos en el año 2, en ambos casos con dos repeticiones en cada tratamiento. El Cuadro 15 presenta un esquema de los tratamientos aplicados para ambos años.

La distribución de los animales para cada tratamiento tuvo en cuenta el tipo de alimentación que recibieron los mismos previamente.

A los efectos de presentar las condiciones en las cuales se desarrolló el ensayo, el Cuadro 16 detalla el manejo de los verdeos según el año de evaluación.

Se utilizaron 10 novillos de la raza Hereford en cada tratamiento (dos repeticiones de cada tratamiento), con 15 meses de edad en promedio al inicio del período experimental (nacidos en la primavera de 2012 y 2013 , para los años 1 y 2 , respectivamente). 
Cuadro 15. Tratamientos experimentales y peso vivo lleno al inicio del ensayo para los años 2014 y 2015.

\begin{tabular}{|c|c|c|c|c|c|}
\hline Tratamiento & 1 & $2^{*}$ & 3 & 4 & 5 \\
\hline Nomenclatura & $\mathrm{T}$ (testigo) & ES & EG & AA & GM \\
\hline Forraje & & & Sorgo & & \\
\hline Suplemento & - & $\begin{array}{l}\text { Expeller } \\
\text { de soja }\end{array}$ & $\begin{array}{l}\text { Expeller } \\
\text { de girasol }\end{array}$ & $\begin{array}{l}\text { Afrechillo } \\
\text { de arroz }\end{array}$ & $\begin{array}{l}\text { Grano } \\
\text { de maíz }\end{array}$ \\
\hline $\begin{array}{l}\text { Nivel de suplementación } \\
(\% \text { PV) }\end{array}$ & - & & & $5 \%$ & \\
\hline PV inicial (kg) - Año 2014 & 269,6 & - & 268,8 & 269,3 & 268,3 \\
\hline PV inicial (kg) - Año 2015 & 265,4 & 265,2 & 265,1 & 265,0 & 265,8 \\
\hline
\end{tabular}

Cuadro 16. Manejo de los verdeos según año de evaluación.

\begin{tabular}{|llc|}
\hline \multicolumn{1}{|c}{ Labor } & \multicolumn{1}{c|}{ Año 2014 (Año 1) } & \multicolumn{1}{c|}{ Año 2015 (Año 2) } \\
\hline Acondicionamiento previo & $\begin{array}{l}\text { 3 L/ha de glifosato potásico } \\
\text { 2.4 D Amina (1 L/ha) }\end{array}$ & $\begin{array}{l}\text { 3 L/ha de glifosato potásico } \\
\text { 2.4 D Amina (1,5 L/ha) }\end{array}$ \\
\hline Material genético & Sorgo forrajero ACA 727 & Sorgo BMR 715 \\
\hline Tratamiento de la semilla & $\begin{array}{l}\text { Protector (Concep) 4 cc/10 kg semilla } \\
\text { Insecticida (Imidaclorpid 60 \% FS) 350 cc/100 kg semilla } \\
\text { Fungicida (Metalaxil 35 \%) 350 cc/100 kg semilla }\end{array}$ \\
\hline Método de siembra & Siembra directa, a 32 cm entre hileras \\
\hline Densidad de siembra & 25 kg/ha & 20 kg/ha \\
\hline Fertilización basal & 100 kg/ha de 25.33.0 & $150 \mathrm{~kg} / \mathrm{ha} 25.33 .0$ \\
\hline Fecha de siembra & 17/10/13 & 23/10/14 \\
\hline Re-fertilización & $50 \mathrm{~kg} / \mathrm{ha} \mathrm{urea}$ & $50 \mathrm{~kg} / \mathrm{ha} \mathrm{urea}$ \\
\hline
\end{tabular}

El sistema de pastoreo utilizado en ambos años fue rotativo, en 3 parcelas, con 10 días de ocupación y 20 días de descanso. Los animales de los tratamientos suplementados eran racionados una vez al día, temprano en la mañana, a razón del 0,5 \% del PV lleno, en forma grupal (comederos). No fue necesario realizar un período de acostumbramiento a los suplementos, debido a que los animales tenían experiencia previa reciente. Los animales contaron con agua de calidad y sales minerales ad libitum durante todo el período experimental. Las determinaciones en el forraje se realizaron de la misma forma en que se describe en la sección 2.1.3.1. Pasturas.

El peso vivo lleno (PVLL) se registró al inicio y al final de cada período experimen- tal y cada 14 días en el año 1 y cada 10 días en el año 2. El peso vivo vacío (PVV) se registró siempre con 16 horas de ayuno, al inicio y al final del período experimental.

El manejo de la sanidad, las determinaciones del comportamiento animal, el ajuste del suplemento ofrecido y la estimación del mismo, se realizaban siguiendo la misma metodología que la descripta en la sección 2.1.3.2. Animales.

Las determinaciones por ultrasonografía fueron tomadas en cuatro oportunidades a lo largo de cada período experimental, según lo descripto en la sección 2.1.3.2. Animales. 


\subsubsection{Diseño experimental}

El diseño experimental utilizado fue completamente al azar con dos repeticiones, en donde se evaluó el efecto de la suplementación. Las variables de pasturas fueron analizadas con el procedimiento GLM y las de origen animal mediante el procedimiento MIXED del paquete estadístico SAS (SAS, 2013). Luego del análisis de varianza (ANOVA), las medias de mínimos cuadrados fueron calculadas para la comparación entre tratamientos con niveles de significancia de $\alpha=0,05$, utilizando la opción PDIFF del LSmeans, cuando los test de $F$ fueron significativos $(P<0,05$ o $P<0,01)$.

\subsubsection{Resultados}

A continuación se presentan los resultados del ensayo, primero discriminado por el año de evaluación y finalmente se realiza un comentario conjunto de los dos años.

\subsubsection{Año 1}

\subsection{Resultados en pasturas}

El Cuadro 17 presenta los resultados obtenidos en el forraje, según el tratamiento aplicado.

Todos los parámetros que describen el forraje cuantitativamente fueron afectados $(P$ $<0,01$ ) por los tratamientos aplicados. La

Cuadro 17. Efecto de la suplementación de novillos sobre sorgo forrajero (Año 1).

\begin{tabular}{|c|c|c|c|c|c|}
\hline Parámetro & $\mathbf{T}$ & EG & AA & GM & $\mathbf{P}$ \\
\hline \multicolumn{6}{|c|}{ OFRECIDO } \\
\hline Altura promedio (cm) & $63.7^{\mathrm{b}}$ & $64,7^{\mathrm{b}}$ & $62,9^{b}$ & $88,5^{a}$ & ** \\
\hline MS promedio (\%) & 19,8 & 19,5 & 19,5 & 20,3 & ns \\
\hline Disponibilidad promedio (kg MS/ha) & $1842,9^{b}$ & $2073,5^{b}$ & $1732,9^{b}$ & $3210,3^{a}$ & $\star *$ \\
\hline \multicolumn{6}{|c|}{ REMANENTE } \\
\hline Altura promedio (cm) & $25,7^{\mathrm{b}}$ & $31,5^{b}$ & $25,5^{b}$ & $57,8^{a}$ & ** \\
\hline MS promedio (\%) & $18,1^{b}$ & $18,5^{a b}$ & $20,8^{a}$ & $18,1^{b}$ & ** \\
\hline Disponibilidad promedio (kg MS/ha) & $898,3^{b}$ & $1036,9^{b}$ & $817,8^{b}$ & $1490,0^{a}$ & ** \\
\hline \multicolumn{6}{|c|}{ UTILIZACIÓN } \\
\hline Utilización (\%) & 54,6 & 51,9 & 54,5 & 52,3 & ns \\
\hline
\end{tabular}

Nota: medias con letras diferentes entre columnas son significativamente diferentes; ${ }^{* *}=\mathrm{P}<0,01$; $\mathrm{MS}=$ contenido de materia seca; $T$ = testigo sin suplementación; $E G$ = suplementación con expeller de girasol; AA = suplementación con afrechillo de arroz; GM = suplementación con grano de maíz.

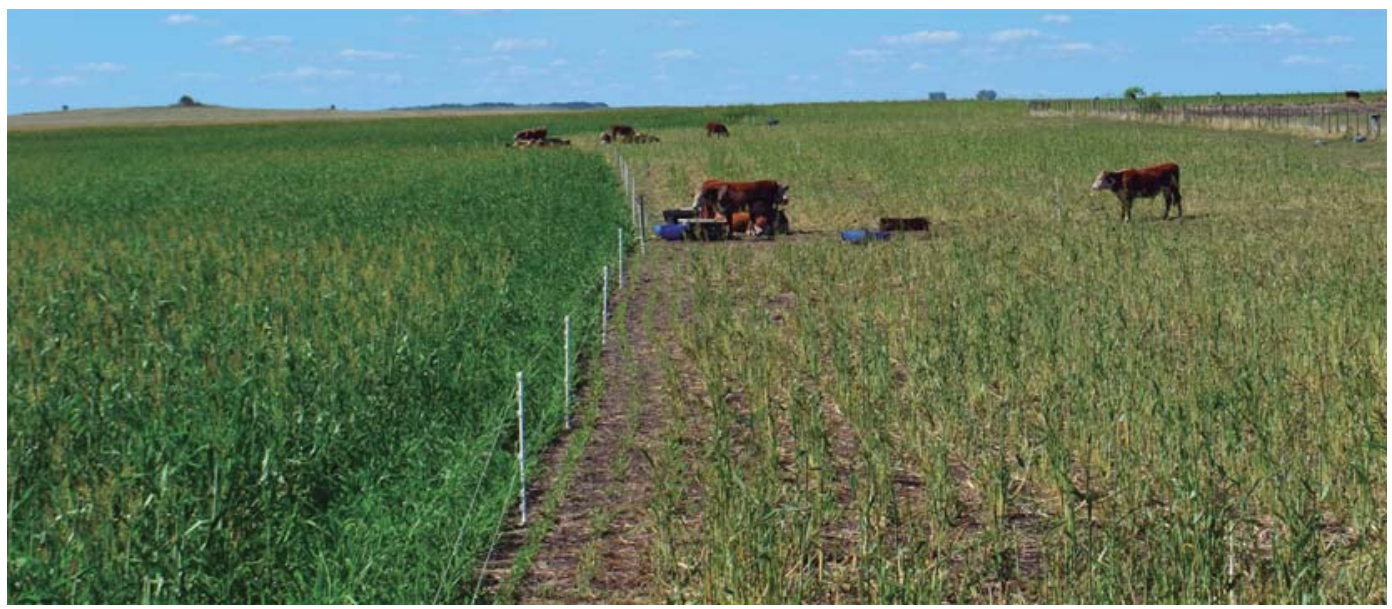


altura y disponibilidad del forraje ofrecido $y$ remanente fueron mayores $(P<0,05)$ en el tratamiento GM respecto a los otros tres tratamientos.

El contenido de materia seca del forraje ofrecido no presentó diferencias $(P>0,05)$ entre tratamientos, mientras que en el forraje remanente los mayores valores fueron registrados en el tratamiento suplementado con AA en comparación con el tratamiento testigo y el suplementado con GM.

En relación a la utilización del forraje, no existieron diferencias significativas $(P>0,05)$ entre los tratamientos. Los valores encontrados son superiores a los reportados en el primer ensayo de esta publicación y se asemejan a las utilizaciones registradas por Vaz Martins et al. (2003), en uno de los híbridos de sorgo evaluados y que fuera pastoreado por novillos, siendo estas de 51 a $53 \%$.

En el Cuadro 18 se presenta el valor nutritivo de la pastura ofrecida y remanente.

Los tratamientos no afectaron significativamente $(P>0,05)$ ninguno de los parámetros asociados al valor nutritivo del forraje ofrecido ni remanente.

Trabajos realizados por Pigurina y Methol (2004) sobre una base de datos de aproximadamente 2000 muestras de sorgo, reportaron niveles promedio de proteína cruda en el forraje ofrecido de $6 \%$. Esquivel et al. (2006) observaron niveles inferiores al $8 \%$ de contenido de este nutriente y Berlangeri (2008) reportó valores entre 12,6 y 13,0 \%. Por su parte, Rovira y Echeverría (2014), evaluaron el contenido de proteína en las diferentes fracciones botánicas, encontrando valores en la fracción hoja de $14,7 \%$ y en la fracción tallo de 5,0 \%.

Se presenta en el Cuadro 19, la composición botánica promedio del sorgo forrajero, según el tratamiento aplicado.

La composición botánica del forraje ofrecido y remanente no fue afectada $(P>0,05)$ por ninguno de los tratamientos experimentales. En general, la principal diferencia en la composición botánica se observa entre el forraje ofrecido y el remanente, donde se detectó un aumento de los restos secos y de la fracción tallo con relación al forraje verde y la hoja. Este proceso puede atribuirse al proceso de selectividad que ejercen los animales durante el pastoreo como ha sido documentado por Montossi (1996) y Montossi et al. (2000).

La Figura 9 presenta la evolución de la composición botánica para el forraje ofrecido, durante todo el período experimental.

Todos los tratamientos fueron evolucionando hacia una menor proporción de hojas

Cuadro 18. Efecto de la suplementación de novillos sobre la calidad del sorgo forrajero (Año 1).

\begin{tabular}{|crlrrr|}
\hline \% & T & \multicolumn{1}{l}{ EG } & AA & GM & P \\
\hline \multicolumn{7}{c}{ OFRECIDO } \\
PC & 8,4 & 10,0 & 9,0 & 8,9 & ns \\
FDA & 38,5 & 37,3 & 38,0 & 38,9 & ns \\
FDN & 66,3 & 63,6 & 64,7 & 64,3 & ns \\
DMS & 58,9 & 59,9 & 59,3 & 58,6 & ns \\
\hline \multicolumn{7}{c}{ REMANENTE } \\
PC & 8,4 & 7,3 & 8,5 & 6,6 & ns \\
FDA & 39,7 & 41,6 & 40,8 & 40,2 & ns \\
FDN & 63,3 & 66,4 & 65,2 & 58,6 & ns \\
DMS & 58,0 & 56,5 & 57,1 & 57,6 & ns \\
\hline
\end{tabular}

Nota: $n s$ = no significativo; $T$ = testigo sin suplementación; $E G$ = suplementación con expeller de girasol; $A A$ = suplementación con afrechillo de arroz; $G M=$ suplementación con grano de maíz; PC = proteína cruda; FDA = fibra detergente ácido; FDN = fibra detergente neutro; DMS = digestibilidad de la materia seca. 
Cuadro 19. Efecto de la suplementación de novillos sobre la composición botánica del sorgo forrajero (Año 1).

\begin{tabular}{|c|c|c|c|c|c|}
\hline$\%$ & $T$ & EG & AA & GM & $\mathbf{P}$ \\
\hline \multicolumn{6}{|c|}{ OFRECIDO } \\
\hline MS verde & 99,6 & 96,0 & 99,1 & 99,2 & ns \\
\hline Hoja & 43,5 & 45,7 & 46,3 & 36,4 & ns \\
\hline Tallo & 49,9 & 40,5 & 53,3 & 62,1 & ns \\
\hline Panoja & 6,6 & 13,7 & 0,4 & 1,4 & ns \\
\hline RS & 0,4 & 6,2 & 1,0 & 0,8 & ns \\
\hline \multicolumn{6}{|c|}{ REMANENTE } \\
\hline MS verde & 95,9 & 97,4 & 89,0 & 95,2 & ns \\
\hline Hoja & 19,0 & 18,6 & 27,9 & 20,4 & ns \\
\hline Tallo & 75,6 & 81,4 & 72,1 & 67,3 & ns \\
\hline Panoja & 5,4 & 0,0 & 0,0 & 12,3 & ns \\
\hline RS & 4,5 & 2,8 & 2,1 & 7,6 & ns \\
\hline
\end{tabular}

Nota: ns = no significativo; $T$ = testigo sin suplementación; $E G$ = suplementación con expeller de girasol; AA = suplementación con afrechillo de arroz; GM = suplementación con grano de maíz MS = contenido de materia seca; RS = fracción restos secos.
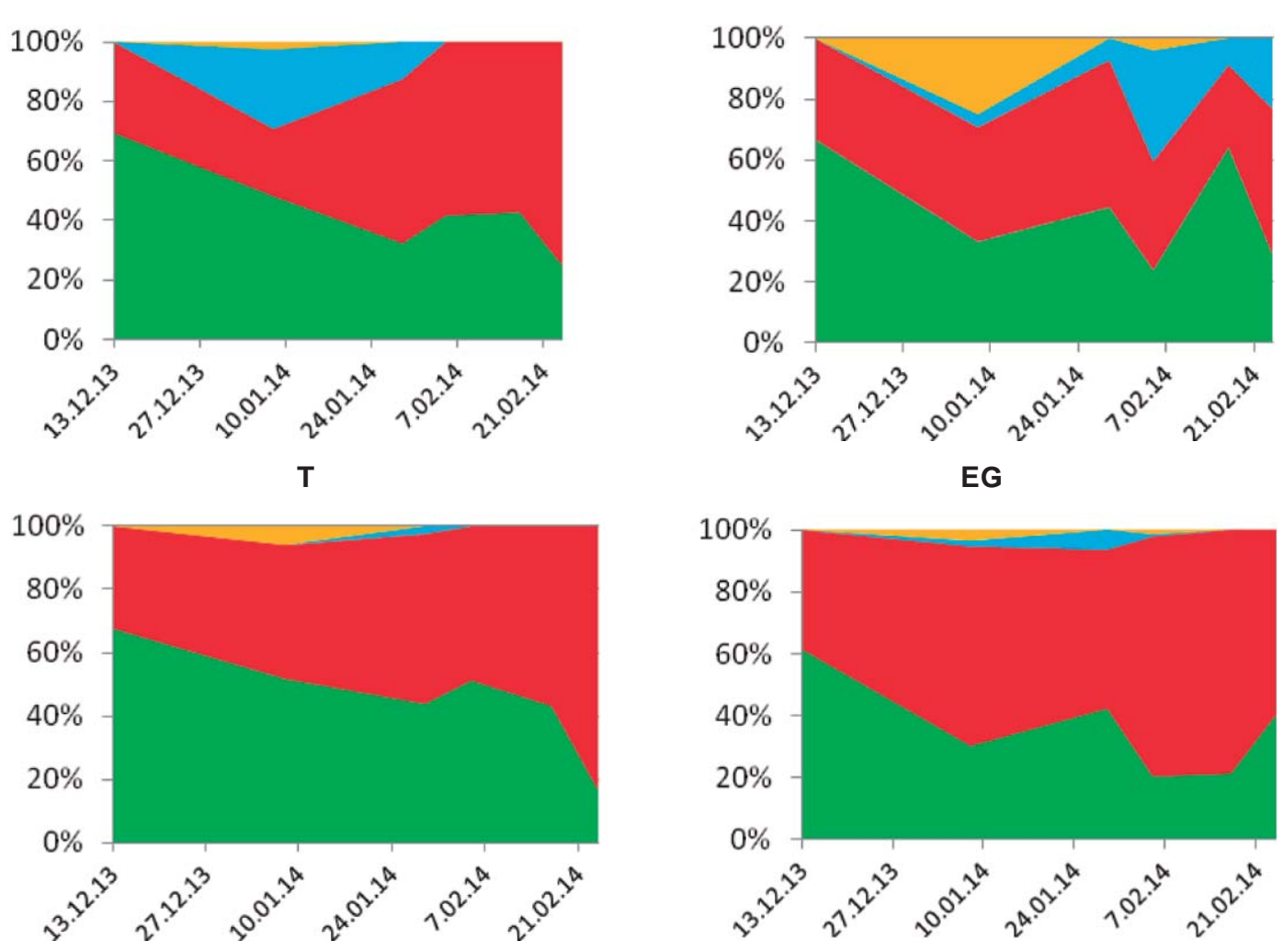

AA

\section{$\square \mathrm{H} \square \mathrm{T} \square \mathrm{P} \square \mathrm{RS}$}

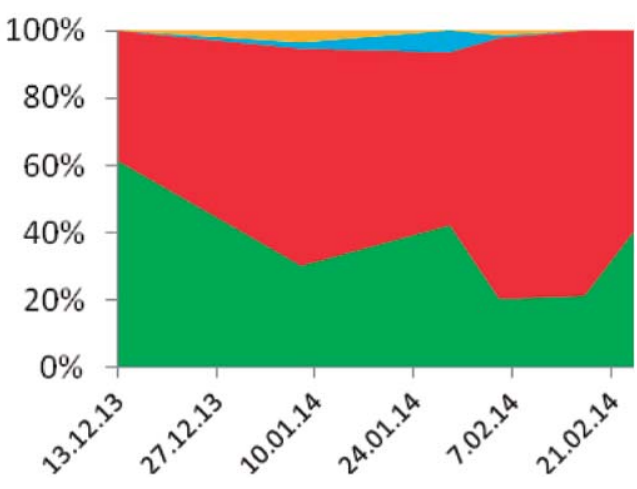

GM

Nota: $T$ = testigo sin suplementación; $E G$ = suplementación con expeller de girasol; $A A$ = suplementación con afrechillo de arroz; GM = suplementación con grano de maíz; $\mathrm{H}=$ hoja; $\mathrm{T}=$ tallo; $\mathrm{P}=$ panoja; $\mathrm{RS}$ = restos secos.

Figura 9. Evolución de la composición botánica del sorgo forrajero ofrecido bajo pastoreo de novillos según tratamiento experimental (Año 1). 


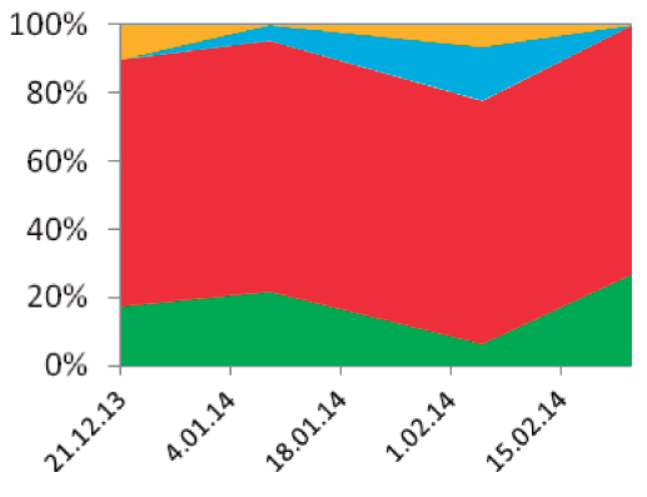

T

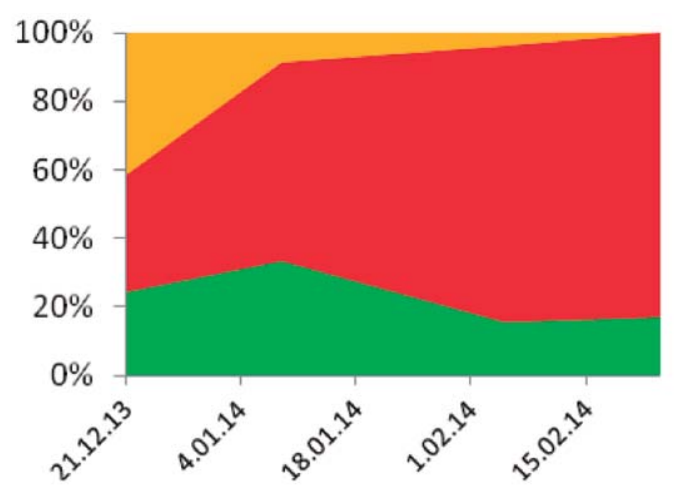

AA

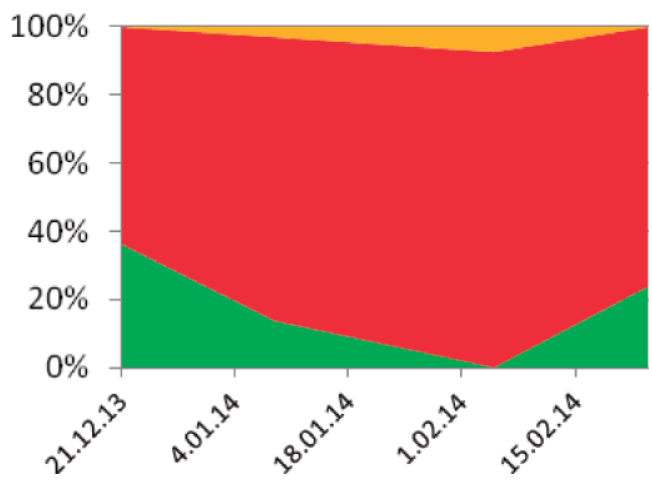

EG

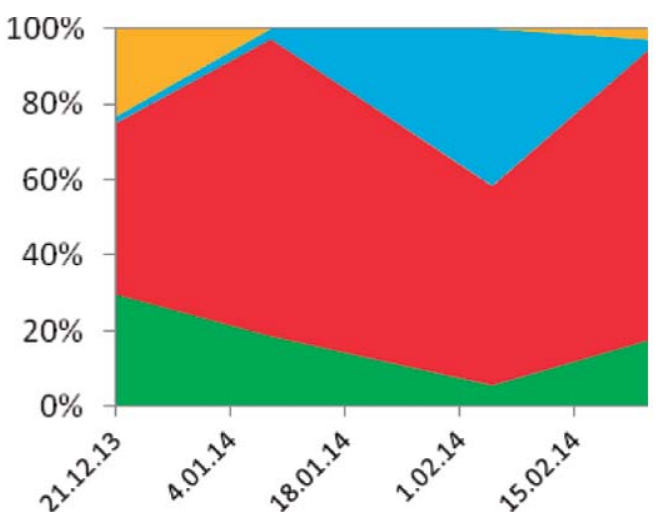

GM

\section{$\square \mathrm{H} \square \mathrm{T} \square \mathrm{P} \square \mathrm{RS}$}

Nota: $\mathrm{T}$ = testigo sin suplementación; $\mathrm{EG}=$ suplementación con expeller de girasol; $\mathrm{AA}=$ suplementación con afrechillo de arroz; $\mathrm{GM}=$ suplementación con grano de maíz; $\mathrm{H}=$ hoja; $\mathrm{T}$ = tallo; $\mathrm{P}=$ panoja; $\mathrm{RS}$ = restos secos.

Figura 10. Evolución de la composición botánica del sorgo forrajero remanente con suplementación bajo pastoreo de novillos (Año 1).

en promedio, con un aumento concomitante de los tallos y de la panoja, en particular para el caso del tratamiento EG.

La Figura 10 presenta la evolución de la composición botánica del forraje remanente, según tratamiento.

Al analizar la evolución de la composición botánica del forraje remanente, independientemente del tratamiento considerado, se observa un bajo aporte de la fracción hoja en relación al componente tallo. En algunos casos la panoja contribuye en una baja proporción como son los casos de GM y T. En los últimos pastoreos, la hoja vuelve a aumentar con respecto a los períodos previos, producto de un rebrote del sorgo.

\subsection{Resultados en comportamiento y producción animal}

Se presentan los registros de conducta animal, según tratamiento, en la Figura 11.

El pastoreo fue la actividad a la que más tiempo le dedicaron los animales, no registrándose diferencias significativas entre tratamientos en este parámetro $(P>0,05)$. Si bien la actividad de rumia presentó los valores más elevados en $\mathrm{T}$, estas diferencias no fueron significativas $(P>0,05)$.

Los tratamientos tuvieron un efecto en las tasas de bocado (TB) $(P<0,05)$ lográndose las mayores $T B$ en el tratamiento $A A$, valores intermedios en $T$ y $E G$ y los menores valores en el tratamiento $\mathrm{GM}$. 


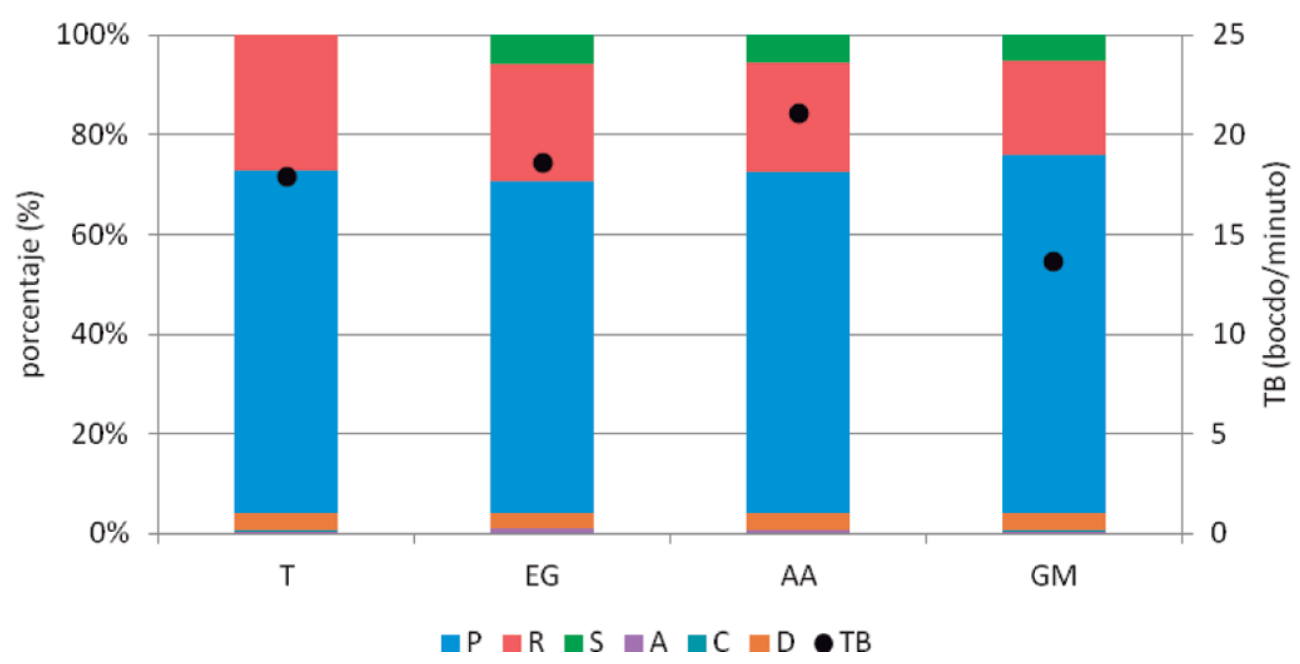

Nota: $T$ = testigo sin suplementación; EG = suplementación con expeller de girasol; $A A$ = suplementación con afrechillo de arroz; $\mathrm{GM}=$ suplementación con grano de maíz; $\mathrm{TB}=$ tasa de bocado; $\mathrm{P}=$ actividad de pastoreo; $\mathrm{R}=$ actividad de rumia; $\mathrm{S}=$ actividad de consumo de suplemento; $\mathrm{A}=$ actividad de consumo de agua; $C$ = actividad de caminata; $D=$ descanso.

Figura 11. Efecto de la suplementación sobre la conducta y tasa de bocado de novillos sobre sorgo forrajero (Año 1).

En el Cuadro 20 se presenta el resumen de los registros de desempeño animal según tratamiento experimental.

El único parámetro que fue afectado por los tratamientos fue la eficiencia de conversión (EC), a favor del tratamiento GM $(P<$ $0,05)$. Esta eficiencia es menor que la regis- trada en el primer y segundo año de evaluación del ensayo presentado previamente en esta publicación, mientras que las registradas para EG y AA son sensiblemente superiores. Esto puede estar explicado por una tasa de sustitución de pastura diferencial por suplemento utilizado y por lo tanto la exis-

Cuadro 20. Efecto de la suplementación sobre la performance animal de novillos sobre sorgo forrajero (Año 1).

\begin{tabular}{|lccccc|}
\hline Parámetro & T & EG & AA & GM & P \\
\hline PVLL i (kg) & 269,6 & 268,8 & 269,3 & 268,3 & ns \\
PVLL f (kg) & 324,8 & 327,0 & 329,8 & 341,3 & ns \\
PVV i (kg) & 262,2 & 256,8 & 257,9 & 257,5 & ns \\
PVV f (kg) & 306,0 & 305,5 & 308,8 & 316,1 & ns \\
AA i (cm) & 119,2 & 119,8 & 119,1 & 118,2 & ns \\
AAN (cm) & 125,2 & 124,6 & 125,3 & 125,6 & ns \\
GPVLL (kg) & 0,521 & 0,549 & 0,571 & 0,689 & ns \\
GPVV (kg) & 0,413 & 0,459 & 0,480 & 0,553 & ns \\
EC (kg S/kg PV) & - & $52,6^{c}$ & $30,0^{b}$ & $9,1^{\text {a }}$ & ** \\
Producción (kg PV/ha) & 414,0 & 436,5 & 453,8 & 547,5 & ns \\
\hline
\end{tabular}

Nota: medias con letras diferentes entre columnas son significativamente diferentes; $\mathrm{ns}=$ no significativo; ${ }^{*}=\mathrm{P}<0,01 ; \mathrm{T}=$ testigo sin suplementación; $\mathrm{EG}=$ suplementación con expeller de girasol; $A A=$ suplementación con afrechillo de arroz; $\mathrm{GM}=$ suplementación con grano de maíz; $\mathrm{i}=$ inicial; $f=$ final; $P V L L=$ peso vivo lleno; $P V V=$ peso vivo vacío; $A A N=$ altura de anca; $G P V=$ ganancia de peso vivo; EC = eficiencia de conversión. 


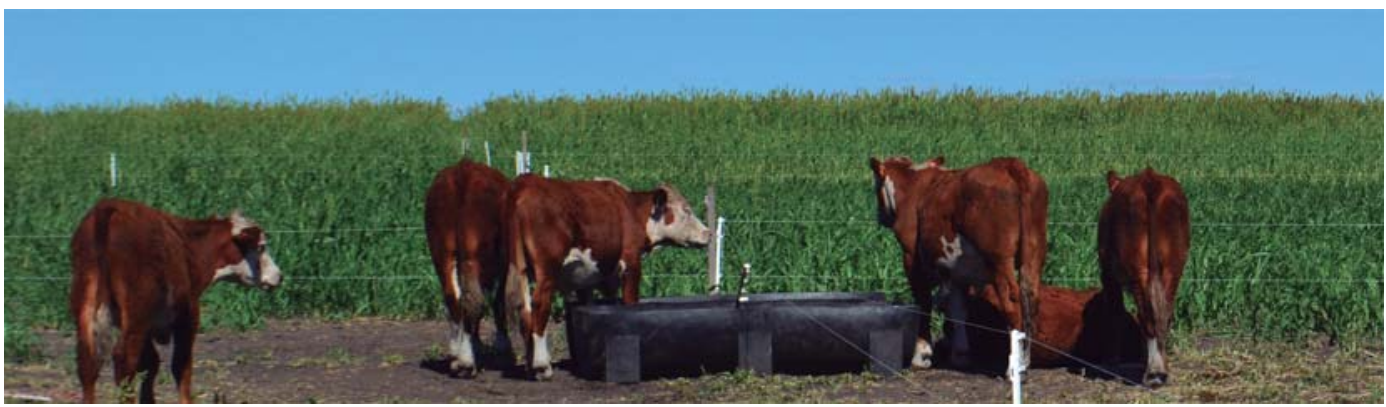

tencia de niveles de disponibilidad de forraje significativamente superiores (Cuadro 17), aunque la disponibilidad del forraje ofrecido promedio fue mayor en el caso de GM, probablemente por una mayor disponibilidad de forraje remanente del pastoreo previo.

La Figura 12 presenta la evolución en el tiempo de los pesos vivos según tratamiento.

Como ya se mencionó, no se registraron diferencias significativas $(P>0,05)$ entre los $\mathrm{PV}$ finales, la gráfica muestra que los tratamientos evolucionaron de manera muy similar a lo largo del mismo si bien al final del mismo el tratamiento suplementado con GM comenzó a destacarse frente a los demás.

En cuanto a la evolución del PV del tratamiento GM, el aumento más marcado hacia el final del período podría estar asociado a un aumento de la fracción hojas del forraje ofrecido e incluso en el remanente.

Se presenta en el Cuadro 21 los datos de calidad de canal in vivo según tratamiento.

Los tratamientos no afectaron $(P>0,05)$ ninguno de los parámetros de calidad de la canal medidos en los animales in vivo.

\subsection{Comentarios y conclusiones del Año 1}

Los tratamientos aplicados no tuvieron un efecto en los parámetros de desempeño animal evaluados ( $P>0,05)$, salvo en la eficiencia de conversión a favor del tratamiento GM. El efecto de sustitución de forraje por el GM, se evidenciaría por la mayor cantidad de forraje promedio disponible y su altura, al igual que para el caso del forraje remanente.

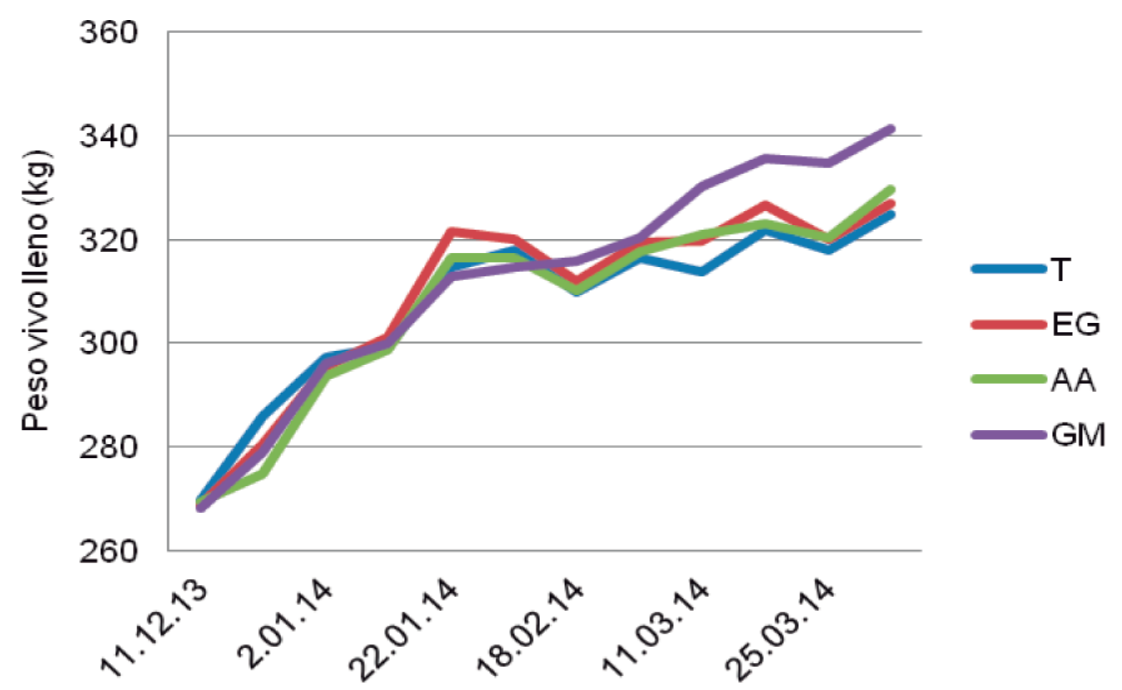

Nota: $T$ = testigo sin suplementación; $E G$ = suplementación con expeller de girasol; $A A$ = suplementación con afrechillo de arroz; GM = suplementación con grano de maíz.

Figura 12. Evolución del peso vivo de novillos suplementados sobre sorgo forrajero (Año 1). 
Cuadro 21. Efecto de la suplementación sobre la calidad de canal de novillos sobre sorgo forrajero (Año 1).

\begin{tabular}{|lrrrrl|}
\hline Parámetro & \multicolumn{1}{c}{ T } & EG & AA & GM & P \\
\hline AOB i $\left(\mathrm{cm}^{2}\right)$ & 38,61 & 36,76 & 35,37 & 37,25 & ns \\
AOB f $\left(\mathrm{cm}^{2}\right)$ & 38,79 & 40,00 & 38,68 & 40,52 & ns \\
EGS i $(\mathrm{mm})$ & 2,31 & 2,16 & 2,26 & 2,10 & ns \\
EGS f $(\mathrm{mm})$ & 2,40 & 2,03 & 2,33 & 2,37 & ns \\
P8 i $(\mathrm{mm})$ & 2,38 & 2,25 & 2,37 & 2,23 & ns \\
P8 f $(\mathrm{mm})$ & 2,65 & 2,20 & 2,19 & 2,46 & ns \\
\hline
\end{tabular}

Nota: medias con letras diferentes entre columnas son significativamente diferentes; ns = no significativo; $\mathrm{T}$ = testigo sin suplementación; $\mathrm{EG}=$ suplementación con expeller de girasol; $A A$ = suplementación con afrechillo de arroz; $G M=$ suplementación con grano de maíz; $\mathrm{i}=$ inicial; $\mathrm{f}$ = final; $\mathrm{AOB}$ = área ojo de bife; $\mathrm{EGS}$ = espesor grasa subcutánea; $\mathrm{P} 8$ = espesor de grasa del cuadril.

También puede mencionarse una evolución (tendencia) de la composición botánica con mayor proporción de hojas en el tratamiento suplementado con GM, especialmente hacia el final del período experimental, lo que se reflejó en las ganancias de peso vivo de los animales hacia el final del período.

Es de destacar que a una única carga de 7,5 novillos/ha y una oferta de suplemento del $0,5 \%$ del PV, no es posible incidir en ganancias de PV diferenciales entre suplementos de una amplia variación en su contenido nutricional, particularmente en el com- ponente PC. Las ganancias de PV estuvieron en un rango de 0,500 a 0,700 kg/an/día, con niveles de productividad por unidad de superficie de 400 a $550 \mathrm{~kg} \mathrm{PV/ha} \mathrm{en} \mathrm{un} \mathrm{pe-}$ ríodo de 106 días. Con las ganancias diarias obtenidas en este experimento no fue posible superar los $350 \mathrm{~kg}$ de PV final, demostrando que las opciones tecnológicas manejadas permiten acelerar el proceso de recría pero no son lo suficientemente importantes como para facilitar la terminación de animales sobre esta base forrajera con el nivel y tipo de suplementos y carga animal empleados.

Cuadro 22. Efecto de la suplementación de novillos sobre sorgo forrajero (Año 2).

\begin{tabular}{|lcccccc|}
\hline Parámetro & T & ES & EG & AA & GM & P \\
\hline \multicolumn{7}{c|}{ OFRECIDO } \\
Altura (cm) & 86,6 & 90,6 & 82,7 & 79,1 & 83,4 & $\mathrm{~ns}$ \\
MS (\%) & 24,3 & 23,7 & 23,4 & 24,2 & 23,3 & $\mathrm{~ns}$ \\
Disponible (kg MS/ha) & 3900,0 & 3958,9 & 3373,3 & 3327,0 & 3342,4 & $\mathrm{~ns}$ \\
\hline \multicolumn{7}{c}{ REMANENTE } \\
Altura (cm) & $63,3^{\text {ab }}$ & $66,9^{\mathrm{a}}$ & $54,3^{\mathrm{b}}$ & $53,1^{\mathrm{b}}$ & $65,6^{\mathrm{a}}$ & $*$ \\
MS (\%) & 22,6 & 20,5 & 22,9 & 22,1 & 21,3 & $\mathrm{~ns}$ \\
Disponible (kg MS/ha) & 1908,4 & 1732,5 & 1587,5 & 1351,6 & 1511,4 & $\mathrm{~ns}$ \\
\hline \multicolumn{7}{c}{ UTILIZACIÓN } \\
Utilización (\%) & 55,7 & 57,8 & 54,2 & 58,3 & 55,8 & $\mathrm{~ns}$ \\
\hline
\end{tabular}

Nota: medias con letras diferentes entre columnas son significativamente diferentes; ${ }^{*}=\mathrm{P}<0,01$; $\mathrm{MS}=$ contenido de materia seca; $\mathrm{T}$ = testigo sin suplementación; $\mathrm{ES}=$ suplementación con expeller de soja; $E G$ = suplementación con expeller de girasol; $A A$ = suplementación con afrechillo de arroz; $\mathrm{GM}$ = suplementación con grano de maíz. 


\subsubsection{Año 2}

\subsection{Resultados en pasturas}

Los resultados registrados en la pastura se presentan en el Cuadro 22.

Salvo en la altura del forraje remanente, ninguno de los parámetros de la pastura fueron afectados $(P>0,05)$ por los tratamientos experimentales.

Las alturas, tanto del forraje ofrecido como del forraje remanente, fueron sensiblemente mayores a las presentadas en el Año 1, lo cual también se vio reflejado en mayores disponibilidades de forraje. De cualquier manera, las alturas del forraje ofrecido están por debajo de las reportadas para el primer y segundo ciclo de pastoreo de la experiencia llevada a cabo por Rovira y Echeverría (2013), quienes registraron 98 y $93 \mathrm{~cm}$, respectivamente.

Al considerar las disponibilidades ofrecidas, en todos los casos se superaron los $3000 \mathrm{~kg} \mathrm{MS} / \mathrm{ha}$. Sin embargo, estas disponibilidades de sorgo BMR están por debajo de lo reportado por Rovira y Echeverría (2013), las que fueron 6845, 9550 y $6935 \mathrm{~kg}$ MS/ha para el primer, segundo y tercer pastoreo, respectivamente.

De la misma manera que en el Año 1, las utilizaciones de forraje no registraron diferen- cias significativas entre tratamientos $(P>$ $0,05)$, si bien en este último caso presentaron valores superiores a los del Año 1. Las eficiencias de utilización del forraje reportadas por Vaz Martins et al. (2003) corresponden a valores de entre $45-53 \%$ para una altura de ingreso a la franja de $60 \mathrm{~cm}$ y 46 $51 \%$ para alturas de ingreso de $100 \mathrm{~cm}$.

El Cuadro 23 presenta los parámetros de calidad del forraje determinados para cada tratamiento.

Al igual que en el Año 1, ninguno de los parámetros del valor nutritivo del forraje ofrecido y remanente fue afectado significativamente $(P>0,05)$ por los tratamientos.

Rovira y Echeverría (2013) registraron contenidos de proteína cruda del orden del $12,9 \%$ en hoja y $3,5 \%$ en tallo. En esta oportunidad, el contenido de proteína cruda del forraje ofrecido está por debajo del contenido reportado por Rovira y Echeverría (2013) para la fracción hoja, resultando en valores similares a los de la fracción tallo. Sin embargo, es importante tener presente que en este trabajo los resultados no se encuentran discriminados en fracción hoja y tallo, sino que es un valor promedio de todas las fracciones del forraje.

Los valores de digestibilidad, tanto del forraje ofrecido como del forraje remanente, se

Cuadro 23. Efecto de la suplementación de novillos sobre los parámetros del valor nutritivo del sorgo forrajero (Año 2).

\begin{tabular}{|c|c|c|c|c|c|c|}
\hline$\%$ & $T$ & ES & EG & AA & GM & $\mathbf{P}$ \\
\hline \multicolumn{7}{|c|}{ OFRECIDO } \\
\hline PC & 8,4 & 9,2 & 8,4 & 8,7 & 7,4 & ns \\
\hline FDA & 50,1 & 50,1 & 49,2 & 50,0 & 48,7 & ns \\
\hline FDN & 64,7 & 60,9 & 64,5 & 63,2 & 65,5 & ns \\
\hline DMS & 49,9 & 49,9 & 50,5 & 49,9 & 50,9 & ns \\
\hline \multicolumn{7}{|c|}{ REMANENTE } \\
\hline PC & 3,2 & 3,4 & 3,2 & 3,4 & 3,3 & ns \\
\hline FDA & 47,8 & 46,2 & 47,5 & 47,4 & 48,1 & ns \\
\hline FDN & 74,6 & 72,9 & 73,9 & 73,8 & 74,6 & ns \\
\hline DMS & 51,7 & 52,9 & 51,9 & 51,9 & 51,5 & ns \\
\hline
\end{tabular}

Nota: $\mathrm{ns}$ = no significativo; $\mathrm{ES}$ = suplementación con expeller de soja; $\mathrm{EG}$ = suplementación con expeller de girasol; $A A=$ suplementación con afrechillo de arroz; $G M=$ suplementación con grano de maíz; $P C=$ proteína cruda; FDA = fibra detergente ácido; FDN = fibra detergente neutro; DMS = digestibilidad de la materia seca. 
encuentran muy por debajo de lo reportado por Rovira y Echeverría (2014) quienes publicaron valores de 67,7 y $70,7 \%$ de digestibilidad in vitro para la fracción hoja y tallo, respectivamente, de un sorgo de pastoreo BMR. Por otro lado, Cummins (1981) citado por Contreras-Govea et al. (2010) reportaron valores de digestibilidad de sorgos con distintos grados de madurez de 38-44 y 53-55 \% para la fracción hoja y tallo, respectivamente. Ambas fuentes citan valores mayores de digestibilidad en la fracción tallo con respecto a la fracción hoja, lo cual podría explicar por qué en este caso, las digestibilidades del forraje ofrecido (mayor contenido de hoja relativo) son menores que las del forraje remanente (mayor contenido de tallo relativo).

Los valores de FDA son mayores que los reportados por Rovira y Echeverría (2014) de 38,8 y $39,4 \%$ para hoja y tallo, respectivamente. De la misma forma, estos autores encontraron valores inferiores de FDN: 63,7 y $60,2 \%$ para hoja y tallo, respectivamente.

El Cuadro 24 presenta los resultados registrados en la composición botánica del sorgo forrajero, según tratamiento.

Ninguno de los parámetros descriptivos de la composición botánica del verdeo fue afectado $(P>0,05)$ por los tratamientos experimentales, tanto para el forraje ofrecido como para el remanente.

Al comparar estos resultados con los del primer año de evaluación, se destaca que en el Año 2, la proporción de la fracción hoja está muy por debajo de la obtenida en el Año 1 , mientras lo inverso sucede con la fracción tallo. En otras palabras, desde el punto de vista de la composición botánica, en esta oportunidad el forraje fue de menor calidad que en el primer año de evaluación.

Al igual que en Año 1, al comparar la composición botanica del forraje ofrecido con respecto al permanente, se observan los efectos de la selectividad (Montossi, 1996; Montossi et al., 2000), favoreciendo la utilización del componente verde y en particular de la fracción hoja en desmedro del resto de los componentes; en particular la fracción tallo de esta opción forrajera.

La Figura 13 presenta la evolución de la composición botánica en el forraje ofrecido, a lo largo del período experimental.

Todos los tratamientos presentaron una mayor proporción de panojas al principio del ensayo, para luego desaparecer. Esto podría indicar que el inicio del pastoreo se realizó

Cuadro 24. Efecto de la suplementación de novillos sobre la composición botánica del sorgo forrajero (Año 2).

\begin{tabular}{|c|c|c|c|c|c|c|}
\hline Parámetro & $\mathbf{T}$ & ES & EG & AA & GM & $\mathbf{P}$ \\
\hline \multicolumn{7}{|c|}{ OFRECIDO (\%) } \\
\hline MS verde & 92,4 & 93,5 & 92,6 & 92,8 & 91,2 & ns \\
\hline Hoja & 28,9 & 24,2 & 25,8 & 25,1 & 22,8 & ns \\
\hline Tallo & 69,8 & 71,0 & 73,8 & 70,8 & 72,6 & ns \\
\hline Panoja & 1,3 & 4,8 & 0,4 & 4,1 & 4,6 & ns \\
\hline RS & 7,6 & 6,5 & 7,4 & 7,2 & 8,8 & ns \\
\hline \multicolumn{7}{|c|}{ REMANENTE (\%) } \\
\hline MS verde & 89,2 & 92,4 & 87,9 & 88,4 & 90,0 & ns \\
\hline Hoja & 4,1 & 3,1 & 2,9 & 2,4 & 1,7 & ns \\
\hline Tallo & 95,9 & 95,3 & 97,1 & 97,6 & 98,3 & ns \\
\hline Panoja & 0,0 & 1,5 & 0,0 & 0,0 & 0,0 & ns \\
\hline RS & 10,8 & 7,6 & 12,1 & 11,6 & 10,0 & ns \\
\hline
\end{tabular}

Nota: $n s$ = no significativo; $T$ = testigo sin suplementación; $E S$ = suplementación con expeller de soja; $E G$ = suplementación con expeller de girasol; $A A$ = suplementación con afrechillo de arroz; $\mathrm{GM}$ = suplementación con grano de maíz; MS = contenido de materia seca; RS = fracción restos secos. 


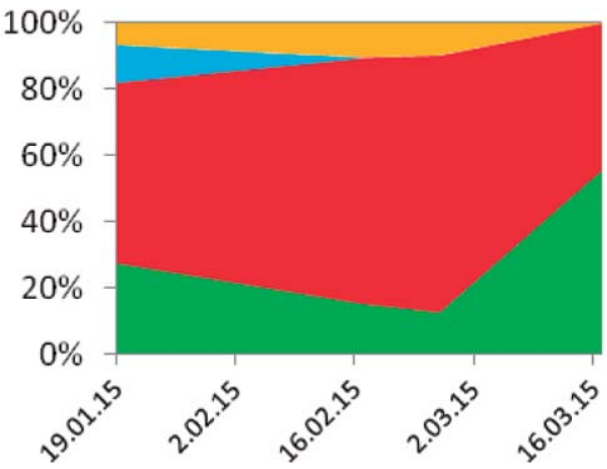

T

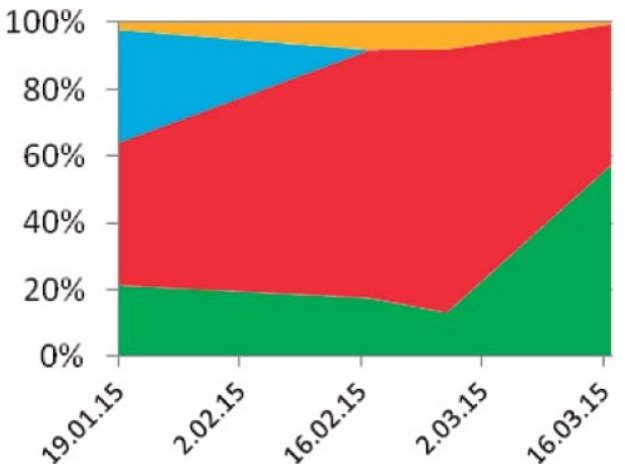

ES
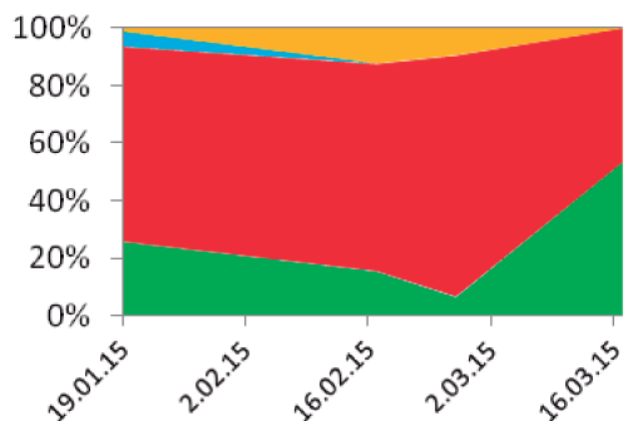

EG
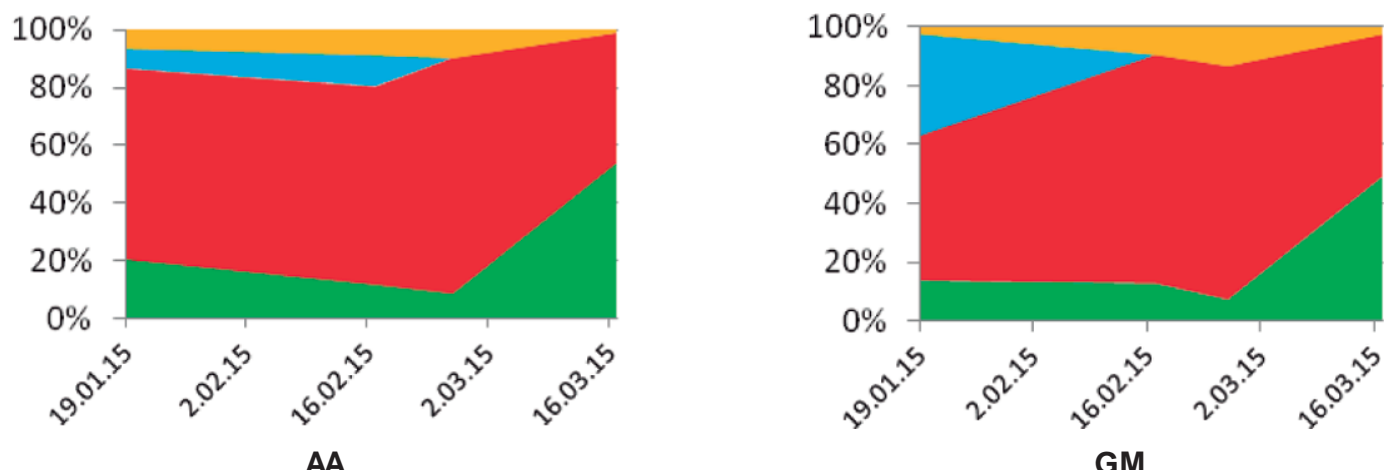

AA

$\square \mathrm{H} \square \mathrm{T} \square \mathrm{P} \square \mathrm{RS}$

Nota: $T$ = testigo sin suplementación; $E S$ = suplementación con expeller de soja; $E G$ = suplementación con expeller de girasol; $A A=$ suplementación con afrechillo de arroz; $G M=$ suplementación con grano de maíz; $\mathrm{H}=$ hoja; $\mathrm{T}$ = tallo; $\mathrm{P}=$ panoja; $\mathrm{RS}$ = restos secos.

Figura 13. Evolución de la composición botánica del sorgo forrajero ofrecido según tratamiento (Año 2).

en un estado vegetativo del sorgo un tanto avanzado. Por otra parte, en el último tercio del período experimental, la fracción hoja aumentó en todos los tratamientos. Esto indicaría que el sorgo fue manejado correctamente desde el punto de vista del control de la floración y de la proporción de hojas en el forraje.

En la Figura 14 se presenta la evolución de la composición botánica del forraje rema- nente del sorgo forrajero, a lo largo del período de evaluación.

Cabe destacar que todos los tratamientos tuvieron una evolución de la composición del forraje remanente muy similar entre sí. A diferencia de lo que se registró en el Año 1, el forraje remanente del Año 2 evolucionó con proporciones muy bajas de la fracción hoja y un predominio total de la fracción tallo. A diferencia del Año 1, los RS estuvieron pre- 

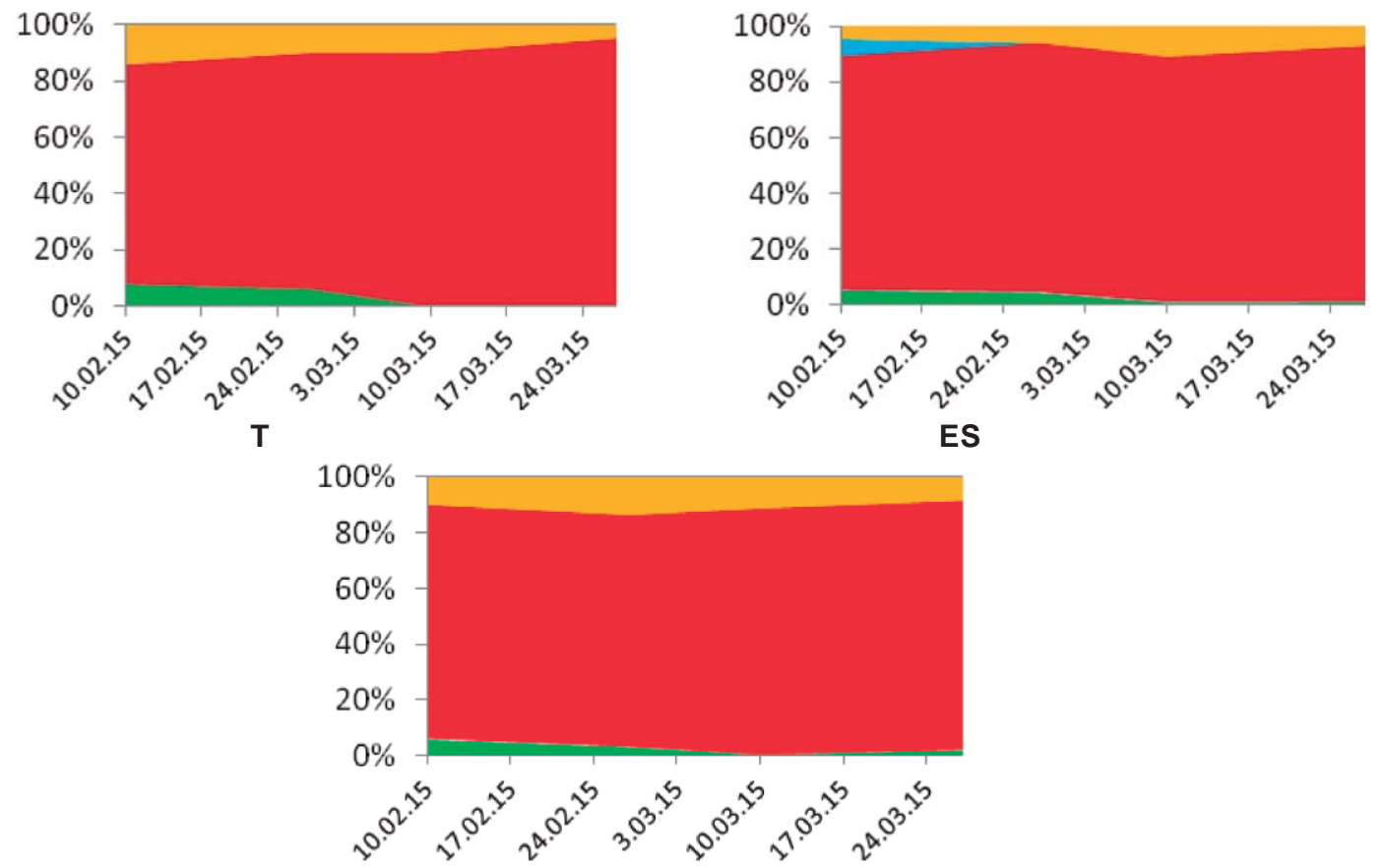

EG

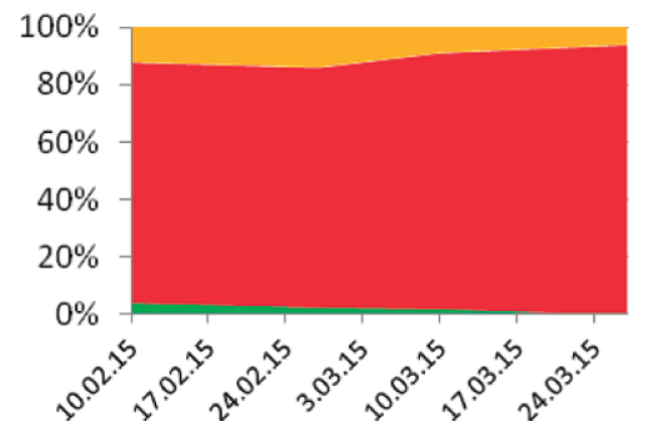

AA

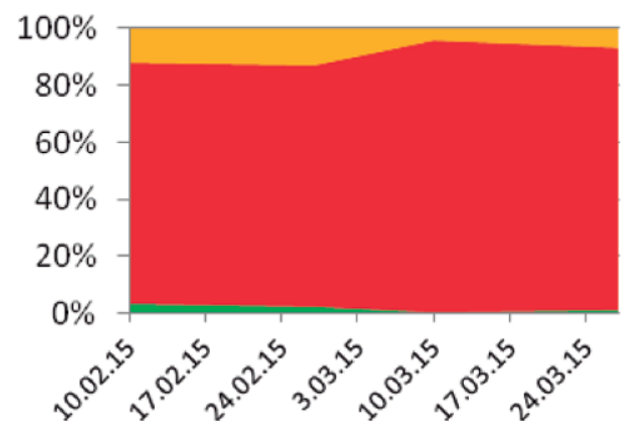

GM

\section{$\mathrm{H} \square \mathrm{T} \square \mathrm{P} \square \mathrm{RS}$}

Nota: $T$ = testigo sin suplementación; $E S$ = suplementación con expeller de soja; EG = suplementación con expeller de girasol; $A A$ = suplementación con afrechillo de arroz; $G M=$ suplementación con gano de maíz; $\mathrm{H}=$ hoja; $\mathrm{T}$ = tallo; $\mathrm{P}=$ panoja; $\mathrm{RS}$ = restos secos.

Figura 14. Evolución de la composición botánica del sorgo forrajero remanente, según tratamiento (Año 2).

sentes a lo largo de todas las evaluaciones realizadas, demostrando un mayor grado de madurez del forraje, que concuerda con la composoción botánica y valor nutricional obtenidos en este estudio.

\subsection{Resultados en comportamiento y producción animal}

En la Figura 15 se presentan los resultados del comportamiento animal, según tratamiento. Al igual que en el primer año de evaluación, el pastoreo fue la actividad con mayor dedicación de tiempo por parte de los animales. Al comparar este parámetro estadísticamente, se encuentra que los tratamientos Ty GM fueron iguales entre sí $(P>0,05)$ y superiores al tratamiento ES $(P<0,05)$, mientras que EG y $A A$ se situaron en una posición intermedia.

Por otra parte, al analizar las tasas de bocado, el tratamiento $T$ logró los valores más elevados, si bien no difirieron significa- 


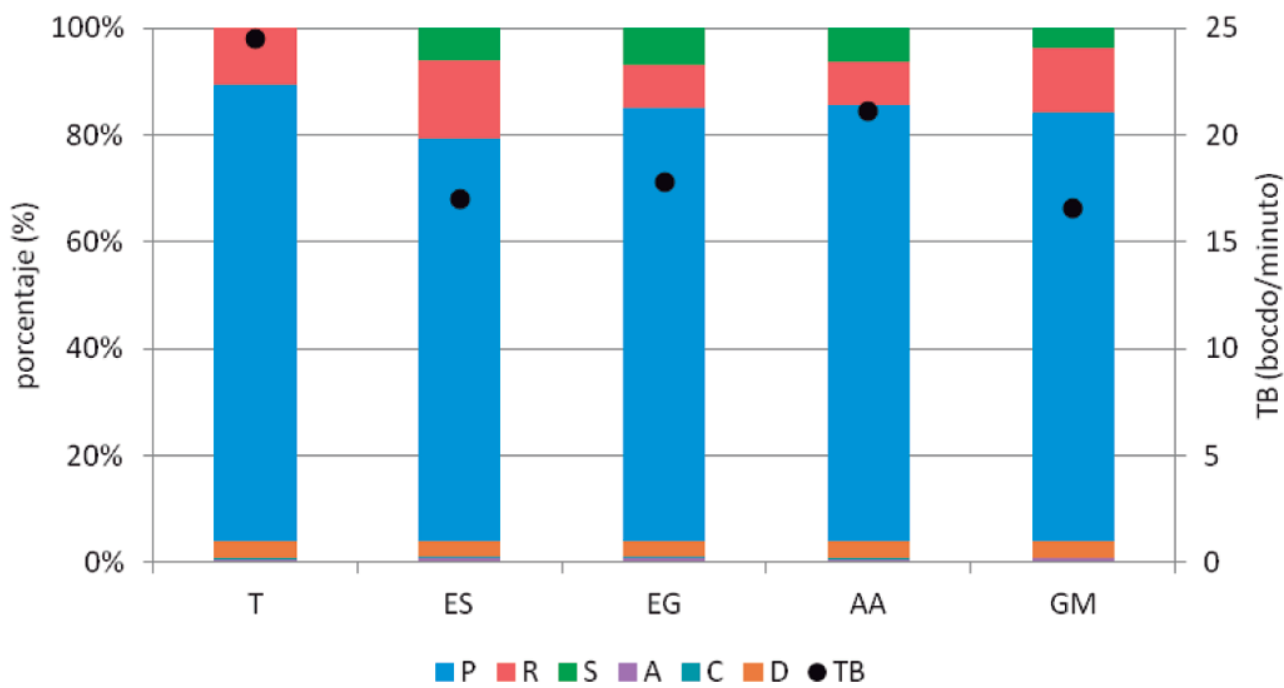

Nota: $T$ = testigo sin suplementación; $E S$ = suplementación con expeller de soja; $E G$ = suplementación con expeller de girasol; AA = suplementación con afrechillo de arroz; GM = suplementación con grano de maíz; $\mathrm{TB}=$ tasa de bocado; $\mathrm{P}=$ actividad de pastoreo; $\mathrm{R}=$ actividad de rumia; $\mathrm{S}=$ actividad de consumo de suplemento; $A$ = actividad de consumo de agua; $C$ = actividad de caminata; $D=$ descanso.

Figura 15. Efecto de la suplementación sobre el comportamiento animal y tasa de bocado, según tratamiento experimental (Año 2).

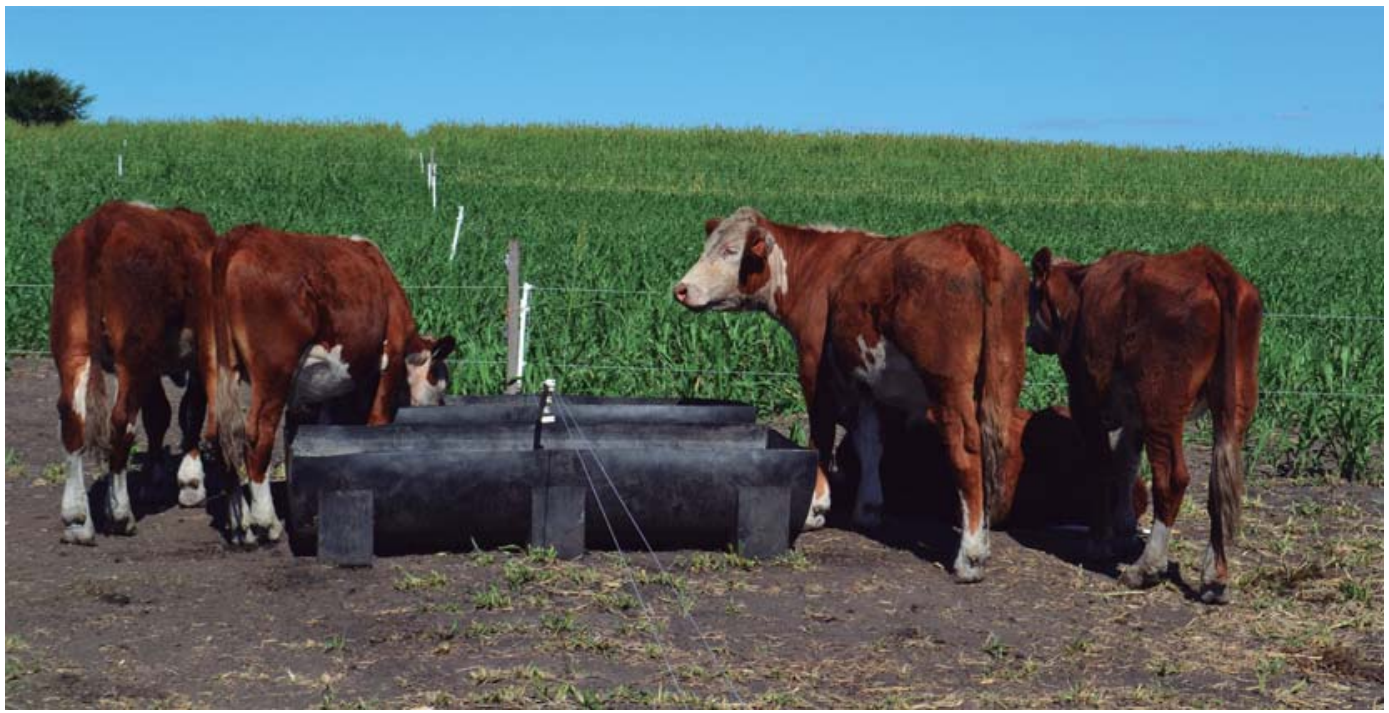

tivamente respecto al tratamiento suplementado con AA; no obstante, los tratamientos ES, EG y GM presentaron tasas de bocado significativamente $(P<0,05)$ menores al tratamiento $\mathrm{T}$. Situaciones más restrictivas para la producción animal en pastoreo resultan en aumentos en la tasa de bocados de bovinos y ovinos (Montossi, 1996).

En el Cuadro 25 se presentan los resultados del desempeño animal.
Es de destacar que no se registraron diferencias significativas $(P>0,05)$ entre tratamientos en los pesos vivos finales, tanto llenos como vacíos. Sin embargo, se registraron diferencias significativas $(P<0,05)$ en la GPVLL, en donde el tratamiento suplementado con GM obtuvo los menores valores productivos respecto a los otros tres tratamientos suplementados. Con respecto al tratamiento GM, en este segundo año de evalua- 
Cuadro 25. Efecto de la suplementación sobre el desempeño animal según tratamiento (Año 2).

\begin{tabular}{|c|c|c|c|c|c|c|}
\hline Parámetro & $\mathrm{T}$ & ES & EG & AA & GM & $\mathbf{P}$ \\
\hline PVLL i (kg) & 265,4 & 265,0 & 265,1 & 265,2 & 265,8 & ns \\
\hline PVLL f (kg) & 313,2 & 323,7 & 324,1 & 321,8 & 311,6 & ns \\
\hline PVV i (kg) & 248,7 & 250,7 & 246,3 & 248,7 & 249,7 & ns \\
\hline$P \vee V f(k g)$ & 297,1 & 315,4 & 311,8 & 304,8 & 294,3 & ns \\
\hline AA i (cm) & 117,3 & 117,2 & 117,2 & 117,3 & 117,3 & ns \\
\hline$A A f(c m)$ & $123,0 \mathrm{ab}$ & $124,1^{\mathrm{ab}}$ & $124,2^{a b}$ & $124,3^{a}$ & $122,7^{b}$ & * \\
\hline GPVLL (kg) & $0,503^{b c}$ & $0,618^{a}$ & $0,621^{a}$ & $0,596^{a b}$ & $0,482^{c}$ & * \\
\hline GPVV (kg) & $0,504^{b}$ & $0,674^{a}$ & $0.682^{a}$ & $0,584^{a b}$ & $0,464^{b}$ & ** \\
\hline EC (kg S/kg PV) & - & $13,0^{b}$ & $12,6^{b}$ & $16,0^{b}$ & $-69,3^{a}$ & ** \\
\hline Producción (kg PV/ha) & $358,5^{b c}$ & $440,3^{a b}$ & $442,5^{a}$ & $424,5^{a b}$ & $343,5^{c}$ & * \\
\hline
\end{tabular}

Nota: medias con letras diferentes entre columnas son significativamente diferentes; ns = no significativo; ${ }^{*}=\mathrm{P}<0,05 ;{ }^{*}=\mathrm{P}<0.01 ; \mathrm{T}=$ testigo sin suplementación; $\mathrm{ES}=$ suplementación con expeller de soja; $E G$ = suplementación con expeller de girasol; $A A$ = suplementación con afrechillo de arroz; $G M$ = suplementación con grano de maíz; i = inicial; $f$ = final; $P V L L$ = peso vivo lleno; $P V V$ = peso vivo vacío; $A A=$ altura de anca; $\mathrm{GPV}=$ ganancia de peso vivo; $\mathrm{EC}=$ eficiencia de conversión.

ción se registró un comportamiento inverso a lo observado en el Año 1, donde si bien las ganancias no fueron significativamente diferentes entre tratamientos, el GM en esa oportunidad presentó una eficiencia de conversión significativamente $(P>0,05)$ más favorable respecto a los otros tratamientos suplementados.
Las ganancias de peso vivo registradas en el presente experimento fueron inferiores a aquellas reportadas por Rovira y Echeverría (2013) de 0,945 kg/an/día en 73 días de evaluación, con una disponibilidad promedio de $8677 \mathrm{kgMS} / \mathrm{ha}$ en un rango de carga anmal de 4,5 a 7 novillos/ha. Estas ganancias también fueron inferiores a las publica-

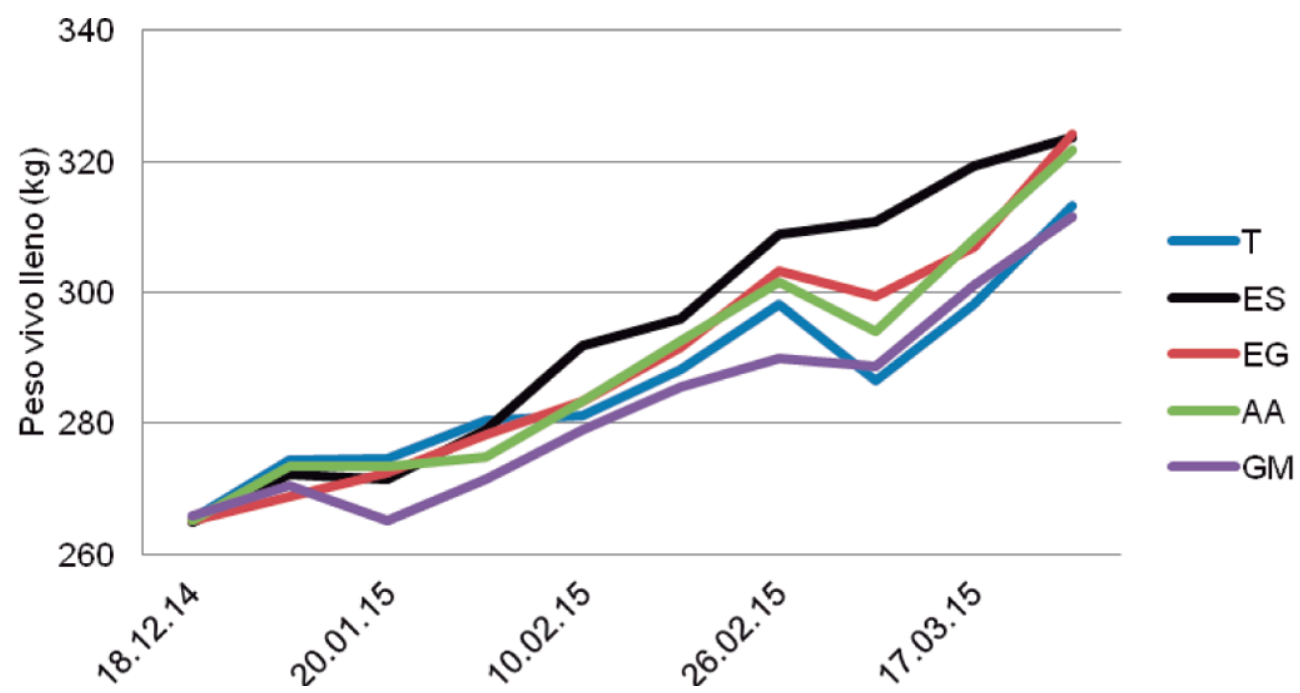

Nota: $\mathrm{T}$ = testigo sin suplementación; $\mathrm{ES}=$ suplementación con expeller de soja; $\mathrm{EG}=$ suplementación con expeller de girasol; AA = suplementación con afrechillo de arroz; GM = suplementación con grano de maíz.

Figura 16. Evolución del peso vivo de novillos pastoreando un sorgo forrajero, según tratamiento (Año 2). 
das por Vaz Martins et al. (2003) quienes reportaron 0,788-0,980 kg/an/día en 79-107 días de evaluación con disponibilidades ubicadas entre 1537 y $1549 \mathrm{~kg}$ MS/ha.

La eficiencia de conversión del tratamiento suplementado con GM fue negativa, ello refleja que los animales suplementados con GM tuvieron ganancias de peso vivo inferiores a las registradas en el tratamiento $T$.

Las EC de los tratamientos ES, EG y AA no fueron significativamente diferentes entre sí $(P>0,05)$, presentando, en general, valores más favorables que los registrados en el primer año.

La Figura 16 presenta la evolución del peso vivo de los animales a lo largo del período experimental.

Desde la segunda pesada, el tratamiento GM quedó rezagado, por debajo de todos los demás tratamientos incluido $\mathrm{T}$, si bien en las últimas tres pesadas estos dos tratamientos ( $T$ y GM) evolucionaron de manera muy similar.

Cabe señalar que el tratamiento de los animales suplementados con ES fue el único cuyo promedio de pesos siempre evolucionó positivamente.

El Cuadro 26 presenta los parámetros de calidad de la canal medidos in vivo.

Las medidas de P8 y GIM iniciales registraron diferencias significativas $(P>0,05)$, a favor de T y GM respecto a ES para P8 y a favor de EG respecto a ES para GIM. No obstante, al finalizar el período experimental, no se observaron diferencias significativas $(P>0,05)$ entre tratamientos en ninguno de los parámetros de calidad de la canal medidos in vivo.

\subsection{Comentarios y conclusiones del Año 2}

El forraje no fue afectado por los tratamientos, en términos de su cantidad y calidad (valor nutritivo y composición botánica). Además, las evoluciones de la composición botánica de cada tratamiento no presentaron mayores diferencias atribuibles a los tratamientos.

Al igual que en el Año 1, se señala que a una única carga de 7,5 novillos/ha y una oferta de suplemento del 0,5\% del PV, no es posible incidir en ganancias de PV diferenciales entre suplementos con una amplia variación en su contenido nutricional, particularmente en el componente de PC. Las ganancias medias se ubicaron en un rango de 0,480 a 0,620 kg/an/día, con niveles de productividad por unidad de superficie de 340 a $440 \mathrm{~kg} \mathrm{PV} / \mathrm{ha}$ en un período de 96 días. Con las ganancias diarias obtenidas en este nuevo experimento no fue posible superar los $320 \mathrm{~kg}$ de $\mathrm{PV}$ final, demostrando que las op-

Cuadro 26. Efecto de la suplementación sobre la calidad de canal y carne determinada in vivo de novillos sobre sorgo forrajero (Año 2).

\begin{tabular}{|c|c|c|c|c|c|c|}
\hline Parámetro & $\mathbf{T}$ & ES & EG & AA & GM & $\mathbf{P}$ \\
\hline AOB i $\left(\mathrm{cm}^{2}\right)$ & 32,15 & 31,78 & 30,37 & 34,16 & 31,84 & ns \\
\hline AOB $\mathrm{f}\left(\mathrm{cm}^{2}\right)$ & 38,25 & 40,78 & 38,12 & 42,21 & 39,41 & ns \\
\hline EGS i (mm) & 1,87 & 1,68 & 1,68 & 1,68 & 1,69 & ns \\
\hline EGS $f(m m)$ & 2,41 & 2,71 & 2,34 & 2,81 & 2,52 & ns \\
\hline P8 i (mm) & $1,97^{\mathrm{a}}$ & $1,63^{b}$ & $1,83^{a b}$ & $1,70^{a b}$ & $1,93^{\mathrm{a}}$ & * \\
\hline$P 8 \mathrm{f}(\mathrm{mm})$ & 3,05 & 3,35 & 2,95 & 3,26 & 3,27 & ns \\
\hline GIM i (\%) & $1,95^{a b}$ & $1,88^{b}$ & $2,22^{a}$ & $1,99 a b$ & $1,90 a b$ & * \\
\hline GIM f (\%) & 2,62 & 2,57 & 2,27 & 2,52 & 2,71 & ns \\
\hline
\end{tabular}

Nota: medias con letras diferentes entre columnas son significativamente diferentes; ns = no significativo; ${ }^{*}=\mathrm{P}<0,05 ; \mathrm{T}=$ testigo sin suplementación; $\mathrm{ES}=$ suplementación con expeller de soja; EG = suplementación con expeller de girasol; $A A=$ suplementación con afrechillo de arroz; $\mathrm{GM}$ = suplementación con grano de maíz; $i$ = inicial; $f$ = final; $A O B=$ área ojo de bife; $E G S$ = espesor grasa subcutánea; P8 = espesor de grasa del cuadril; GIM = grasa intramuscular. 
ciones tecnológicas manejadas aceleran el proceso de recría, pero no son lo suficientemente importantes para facilitar la terminación de animales sobre esta base forrajera con el nivel y tipo de suplementos empleados y partiendo de PV iniciales de $260 \mathrm{~kg}$.

La respuesta animal obtenida entre las diferentes variantes de suplementos fue diferente a la obtenida en el Año 1. En el Año 2 el valor nutricional del forraje fue inferior en contenido de PC, la eficiencia de conversión fue desfavorable para el GM, mientras que las otras alternativas con mayor nivel de contenido proteico ( $A A, E S$ y $E G$ ) fueron más favorables.

\subsubsection{Comentarios y conclusiones de ambos años $(1+2)$ en conjunto}

Al analizar los dos años en conjunto, se puede decir que tanto la producción vegetal como la animal fue muy afectada por el año de evaluación. Por ejemplo, en el Año 1 la cantidad de forraje ofrecido y remanente fue afectado significativamente $(P<0,05)$ por los tratamientos, mientras que en el Año 2 solamente la altura del forraje remanente fue afectada.

El valor nutritivo y la composición botánica no fueron afectados por los tratamientos en ninguno de los dos años. Además, al comparar los valores de la composición botánica y valor nutricional del forraje ofrecido, y particularmente el remanente, se observa que estos fueron superiores para la producción animal en el primer año.

Las ganancias medias diarias oscilaron entre $0,500-0,700$ y 0,480-0,600 kg/an/día para el Año 1 y Año 2, respectivamente. Sobre sorgos forrajeros, cabe señalar que los valores alcanzados en desempeño animal de los presentes trabajos realizados sobre suelos de Basalto fueron menores a los reportados por la bibliografía nacional (Rovira y Echeverría, 2013; Vaz Martins et al., 2003; Berlangeri, 2008). Asimismo, también es importante tener en cuenta que para la mayoría de los casos la carga animal utilizada fue menor a la de los trabajos presentados en esta publicación y que -en general- tam- bién están asociados a niveles de disponibilidad de forraje mayores.

En términos de producción animal por unidad de superficie, en períodos cercanos a los tres meses de utilización de forraje de sorgos durante el verano, la producción varió entre 400 a $550 \mathrm{~kg}$ PV/ha y 340 a $440 \mathrm{~kg}$ $\mathrm{PV} /$ ha para el Año 1 y Año 2, respectivamente.

A la carga ( 7,5 novillos/ha) y niveles de oferta de suplemento (0,5\% de PV) manejados, la suplementación no significó un aumento sustancial en la ganancia diaria de peso vivo con respecto a los animales que no recibieron suplemento. Sin embargo, en términos generales, la suplementación permitió mayor productividad por hectárea. Los coeficientes de eficiencia de conversión de suplementos en PV logrados señalan que las condiciones de suplementación no fueron óptimas para utilizar esta tecnología. Ello puede deberse a una serie de factores asociados, principalmente a las características de la base forrajera utilizada y el manejo animal empleado, donde potencialmente las restricciones no fueron lo suficientemente severas para mejorar la eficiencia y la dimensión de la respuesta a la suplementación en la producción animal esperada.

En cuanto a las diferentes alternativas de suplementos evaluadas, se observó una situación dispar entre años para el caso del $\mathrm{GM}$, donde en condiciones más restrictivas de niveles de proteína en el forraje de base su respuesta es limitada. En cuanto a las diferentes opciones de suplementos con mayores niveles de proteína (AA, EG y ES), los resultados son más consistentes entre años, pero sin encontrar importantes diferencias de respuesta entre las diferentes fuentes consideradas en estos experimentos.

La suplementación establecida en estos trabajos experimentales demuestra claramente que el uso de suplementos no tiene implicancias mayores en la deposición de los diferentes tejidos (músculo y grasa) en esta categoría animal.

Como consideración general, es claro que los sorgos forrajeros permiten sostener altas cargas instantáneas por hectárea. No 
obstante, su valor nutritivo es adecuado para favorecer la recría con ganancias moderadas para novillos de sobre año por un período corto de tiempo. Por otro lado, la suplementación en estas condiciones permite aumentar la capacidad de carga del sistema de recría en su conjunto más que la producción individual.

La viabilidad económica de esta alternativa no solo debe ser evaluada durante el período en la cual se aplica, sino en su impacto en todo el sistema productivo, como lo demuestran las modelaciones bio-económicas realizadas por Montossi et al. (2014) para los sistemas ganaderos del Basalto.

\subsection{Evaluación del uso de diferentes suplementos y cargas sobre sorgo forrajero}

Esta línea de trabajo experimental tuvo por objetivo principal evaluar el pastoreo del sorgo forrajero bajo diferentes cargas y tipos de suplementos como alternativas para acelerar la recría y/o el engorde de novillos durante el período estival, en la región de Basalto.

Los objetivos específicos fueron:

- Evaluar el desempeño animal de novillos Hereford sobre un verdeo de sorgo forrajero a una carga de 7,5 novillos/ha.

- Evaluar el desempeño animal de novillos Hereford sobre un verdeo de sorgo forrajero a una carga de 10 novillos/ha.
- Evaluar el efecto de la suplementación con expeller de girasol (EG) al 0,5\% del peso vivo (PV) en la productividad animal, sobre un verdeo de sorgo forrajero.

- Evaluar el efecto de la suplementación con afrechillo de arroz (AA) al 0,5\% del peso vivo (PV) en la productividad animal, sobre un verdeo de sorgo forrajero.

- Evaluar el efecto de la suplementación con grano de maíz (GM) al 0,5\% del peso vivo (PV) en la productividad animal, sobre un verdeo de sorgo forrajero.

- Evaluar el efecto de la suplementación en la productividad de un verdeo de sorgo forrajero.

- Evaluar el efecto de los tratamientos sobre la deposición de tejidos en el animal in vivo.

- Evaluar el efecto interactivo de los factores evaluados.

\subsubsection{Materiales y métodos}

El período experimental comenzó el 23 de diciembre de 2013 y finalizó 28 de marzo de 2014 (95 días).

Se evaluaron 2 cargas animales (7,5 y 10 novillos/ha) sobre un sorgo forrajero, combinando 3 tipos de suplemento y el testigo sin suplementación, con dos repeticiones por cada tratamiento. El Cuadro 27 detalla los tratamientos aplicados.

Cuadro 27. Descripción de los tratamientos experimentales aplicados y peso vivo lleno al inicio del ensayo.

\begin{tabular}{|c|c|c|c|c|c|c|c|c|}
\hline Tratamiento & 1 & 2 & 3 & 4 & 5 & 6 & 7 & 8 \\
\hline Nomenclatura & $\mathbf{T}$ & EG & AA & GM & $\mathbf{T}$ & EG & AA & GM \\
\hline Carga & & 7,5 novi & illos/ha & & & 10 novil & illos/ha & \\
\hline Pastura & \multicolumn{8}{|c|}{ Sorgo BMR } \\
\hline Suplemento & - & $\begin{array}{c}\text { Expeller } \\
\text { de gira- } \\
\text { sol }\end{array}$ & $\begin{array}{l}\text { Afrechi- } \\
\text { llo de } \\
\text { arroz }\end{array}$ & $\begin{array}{c}\text { Grano } \\
\text { de maíz }\end{array}$ & - & $\begin{array}{c}\text { Expeller } \\
\text { de gira- } \\
\text { sol }\end{array}$ & $\begin{array}{c}\text { Afrechi- } \\
\text { llo de } \\
\text { arroz }\end{array}$ & $\begin{array}{c}\text { Grano } \\
\text { de maíz }\end{array}$ \\
\hline$\%$ PV de suplemento & - & & 0,5 & & - & & 0,5 & \\
\hline PV inicial (kg) & 237,8 & 237,0 & 236,5 & 236,3 & 237,0 & 236,6 & 237,0 & 237,6 \\
\hline
\end{tabular}

Nota: $\mathrm{T}$ = testigo sin suplementación; ES = suplementación con expeller de soja; EG = suplementación con expeller de girasol; AA = suplementación con afrechillo de arroz; GM = suplementación con grano de maíz. 
El manejo de los verdeos fue el mismo que el descrito en el Cuadro 16, dentro de la sección 3.1.1. Materiales y métodos (Año 1).

Se trabajó con un total de 64 novillos Hereford de 15 meses de edad al inicio del período experimental (nacidos en la primavera de 2012).

El sistema de pastoreo, la suplementación, el suministro de agua y sales fueron iguales a los descritos en la sección 3.1.1. Materiales y métodos. Las determinaciones en la base forrajera, así como todas las determinaciones registradas sobre los animales, también fueron descritas la sección previamente mencionada. De todas maneras, es importante señalar que en este caso los pesos vivos llenos se tomaron cada 14 días y que se realizaron en tres instancias mediciones de peso vivo vacío (inicio, medio y final del período experimental).

\subsubsection{Diseño experimental}

El diseño experimental utilizado fue uno factorial de $2 \times 4$ completamente aleatorizado con dos repeticiones. Un factor estuvo representado por la carga animal (7,5 y 10 novillos/ha) y el otro por la suplementación (testigo sin suplementar, expeller de girasol, afrechillo de arroz y grano de maíz). Las variables fueron analizadas mediante un modelo mixto utilizando el procedimiento MIXED del paquete estadístico SAS (SAS, 2013).

\subsubsection{Resultados}

\subsubsection{Resultados en las pasturas}

El Cuadro 28 presenta los resultados registrados en la pastura.

Las disponibilidades del forraje ofrecido y remanente estuvieron dentro de los rangos de los ensayos anteriormente presentados en esta misma publicación. En contraste, Berlangeri (2008) estimó disponibilidades de 11152 y $9918 \mathrm{~kg} \mathrm{MS} / \mathrm{ha}$ con un material de sorgo BMR en el primer pastoreo con noviIlos Holando, mientras que Rovira y Echeverría (2013) estimaron disponibilidades ofrecidas de 6845, 9550 y $9635 \mathrm{~kg} \mathrm{MS} / \mathrm{ha}$ para el primer, segundo y tercer ciclo de pastoreo de sorgo BMR con alturas de 98, 93 y 82 $\mathrm{cm}$, respectivamente.

Al contrastar las dos cargas utilizadas (7,5 y 10 novillos/ha) la disponibilidad, tanto del forraje ofrecido como del remanente, y

Cuadro 28. Efecto de la carga animal y suplementación de novillos pastoreando sorgo forrajero sobre las características de la pastura.

\begin{tabular}{|c|c|c|c|c|c|c|c|c|c|}
\hline \multirow{2}{*}{ Parámetro } & \multicolumn{2}{|c|}{ Carga } & \multirow{2}{*}{$\mathbf{P}$} & \multicolumn{4}{|c|}{ Suplementación } & \multirow{2}{*}{$\mathbf{P}$} & \multirow{2}{*}{$\begin{array}{c}P \\
C * S\end{array}$} \\
\hline & Baja & Alta & & $\mathbf{T}$ & EG & AA & GM & & \\
\hline \multicolumn{10}{|c|}{ OFRECIDO } \\
\hline Altura (cm) & 70,9 & 68,7 & ns & 66,6 & 68,2 & 70,9 & 73,4 & ns & ns \\
\hline MS (\%) & 19,7 & 19,7 & ns & 19,6 & 19,2 & 20,2 & 19,8 & ns & ** \\
\hline $\begin{array}{l}\text { Disponible } \\
\text { (kg MS/ha) }\end{array}$ & 2443,3 & 2188,9 & ns & 2310,1 & 2342,0 & 2335,7 & 2276,6 & ns & ns \\
\hline \multicolumn{10}{|c|}{ REMANENTE } \\
\hline Altura (cm) & 48,8 & 45,2 & ns & 48,3 & 47,0 & 45,5 & 47,2 & ns & ns \\
\hline MS (\%) & 19,6 & 20,0 & ns & $18,6^{c}$ & 20,2 ab & 19,1 bc & $21,4^{a}$ & ** & ns \\
\hline $\begin{array}{l}\text { Disponible } \\
\text { (kg MS/ha) }\end{array}$ & 1154,4 & 1128,3 & ns & 1158,9 & 1200,7 & 1041,3 & 1164,5 & ns & * \\
\hline \multicolumn{10}{|c|}{ UTILIZACIÓN } \\
\hline Utilización (\%) & 45,0 & 50,6 & ns & 46,4 & 47,6 & 52,1 & 45,1 & ns & ns \\
\hline
\end{tabular}

Nota: medias con letras diferentes entre columnas son significativamente diferentes; ${ }^{*}=\mathrm{P}<0,05$; ${ }^{*}=\mathrm{P}<$ 0,01; T = testigo sin suplementación; EG = suplementación con expeller de girasol; AA = suplementación con afrechillo de arroz; GM = suplementación con grano de maíz; Carga baja = 7,5 novillos/ha; Carga alta = 10 novillos/ha; $\mathrm{C}$ = carga; $\mathrm{S}$ = Suplemento; $\mathrm{MS}$ = contenido de materia seca. 
su correspondiente altura, no se vieron afectadas por el factor carga animal.

Por su parte, la suplementación determinó diferencias significativas $(P<0,05)$ únicamente en el \% MS del forraje remanente, siendo menor en el tratamiento que no recibió ningún tipo de suplemento en comparación con los tratamientos suplementados con EG y GM. Las eficiencias de utilización del sorgo forrajero no fueron afectadas $(P>0,05)$ por la carga animal ni por la suplementación.

La interacción entre los dos factores de estudio fue significativa $(P<0,05)$ para $\%$ MS de ambos forrajes analizados y en la disponibilidad de forraje remanente (Cuadro 29).

En el Cuadro 30 se presentan los parámetros de calidad de la pastura, según carga animal y tipo de suplementación.

Cuadro 29. Efecto de la interacción de carga por suplementación en novillos pastoreando sorgo forrajero sobre las características de la pastura.

\begin{tabular}{|c|c|c|c|c|c|c|c|c|c|}
\hline \multirow{2}{*}{ Parámetro } & \multicolumn{4}{|c|}{7,5 novillos/ha } & \multicolumn{4}{|c|}{10 novillos/ha } & \multirow{2}{*}{$\mathbf{P}$} \\
\hline & $\mathbf{T}$ & EG & $A A$ & GM & $\mathbf{T}$ & EG & AA & GM & \\
\hline \multicolumn{10}{|c|}{ OFRECIDO } \\
\hline MS (\%) & $19,6^{b}$ & $19,2^{b}$ & $20,2^{a}$ & $19,8^{b}$ & $19,7^{\mathrm{b}}$ & $18,7^{b}$ & $18,8^{b}$ & $21,4^{a}$ & ** \\
\hline \multicolumn{10}{|c|}{ REMANENTE } \\
\hline MS (\%) & 18,9 ab & $19,4^{a b}$ & 18,8 ab & $21,4^{a}$ & $18,3^{b}$ & $21,0^{a}$ & $19,5^{a b}$ & $21,3^{a}$ & ** \\
\hline $\begin{array}{l}\text { Disponible } \\
\text { (kg MS/ha) }\end{array}$ & $1359,0^{a}$ & 1125,3 ab & $939,2^{b}$ & 1194,2 ab & $958,9^{b}$ & $1276,1^{a}$ & $1143,4{ }^{a b}$ & 1134,8 ab & * \\
\hline
\end{tabular}

Nota: medias con letras diferentes entre columnas son significativamente diferentes; * $=\mathrm{P}<0,05$; ${ }^{*}=\mathrm{P}<$ 0,$01 ; T=$ testigo sin suplementación; $E G$ = suplementación con expeller de girasol; $A A=$ suplementación con afrechillo de arroz; GM = suplementación con grano de maíz; MS = contenido de materia seca.

Cuadro 30. Efecto de la carga y suplementación de novillos sobre los parámetros de calidad del sorgo forrajero.

\begin{tabular}{|c|c|c|c|c|c|c|c|c|c|}
\hline \multirow{2}{*}{$\%$} & \multicolumn{2}{|c|}{ Carga } & \multirow{2}{*}{$\mathbf{P}$} & \multicolumn{4}{|c|}{ Suplementación } & \multirow{2}{*}{$\mathbf{P}$} & \multirow{2}{*}{$\begin{array}{l}P \\
C * S\end{array}$} \\
\hline & Baja & Alta & & $T$ & EG & $A A$ & GM & & \\
\hline \multicolumn{10}{|c|}{ OFRECIDO } \\
\hline PC & 8,4 & 9,1 & ns & 7,9 & 10,3 & 8,5 & 8,3 & ns & ns \\
\hline FDA & 35,6 & 35,9 & ns & $37,4^{a}$ & $34,2^{b}$ & $35,8^{a b}$ & $35,8^{a b}$ & * & * \\
\hline FDN & 61,8 & 62,6 & ns & 63,0 & 60,5 & 62,8 & 62,6 & ns & * \\
\hline C & 10,7 & 11,6 & ns & 12,2 & 11,4 & 10,4 & 10,6 & ns & ns \\
\hline DMS & 66,3 & 65,2 & ns & 67,3 & 66,0 & 65,0 & 64,7 & ns & ns \\
\hline \multicolumn{10}{|c|}{ REMANENTE } \\
\hline PC & 4,7 & 4,0 & ns & 4,7 & 4,0 & 4,3 & 4,5 & ns & ns \\
\hline FDA & 42,0 & 45,0 & ns & 42,4 & 42,3 & 42,7 & 45,3 & ns & ns \\
\hline FDN & $66,2^{b}$ & $71,0^{a}$ & * & 66,5 & 67,8 & 68,6 & 71,5 & ns & ns \\
\hline C & 8,3 & 8,9 & ns & 9,3 & 8,0 & 8,7 & 8,6 & ns & ns \\
\hline DMS & 66,5 & 61,8 & ns & 65,5 & 64,2 & 63,6 & 61,4 & ns & ns \\
\hline
\end{tabular}

Nota: $n s=$ no significativo; * $=\mathrm{P}<0,05 ; \mathrm{ES}=$ suplementación con expeller de soja; $\mathrm{EG}$ = suplementación con expeller de girasol; $\mathrm{AA}=$ suplementación con afrechillo de arroz; $\mathrm{GM}=$ suplementación con grano de maíz; Carga baja = 7,5 novillos/ha; Carga alta = 10 novillos/ha; $P C=$ proteína cruda; $F D A=$ fibra detergente ácido; FDN = fibra detergente neutro; $C$ = cenizas; DMS = digestibilidad de la materia seca. 
A excepción del porcentaje de FDA, ninguno de los parámetros descriptivos del valor nutritivo fue afectado $(P>0,05)$ por la carga animal ni por la suplementación en el forraje ofrecido. Rovira y Echeverría (2014) reportaron valores de FDA de 38,8-39,4\% para hoja y tallo, respectivamente. Estos autores reportaron contenidos de FDN similares a los obtenidos en el presente trabajo sobre el forraje ofrecido, del orden de 60,2$63,7 \%$. La carga animal tuvo un efecto significativo $(P<0,05)$ en el porcentaje de FDN en el forraje remanente, presentando un mayor valor la carga de 10 novillos/ha.
La digestibilidad tanto del forraje ofrecido como del remanente, fue mayor a los valores reportados anteriormente en esta misma publicación. Sin embargo, los valores presentados en el Cuadro 30 son inferiores a las digestibilidades de $67,7-70,7 \%$ reportadas por Rovira y Echeverría (2014).

Los valores de proteína cruda en el presente trabajo se encontraron por encima del $6 \%$ reportado por Pigurina y Methol (2004), aunque están por debajo del 12,6-13,0 \% haIlado por Berlangeri (2008).

La interacción entre factores determinó diferencias significativas $(P<0,05)$ en el

Cuadro 31. Interacción significativa de carga por suplementación en novillos sobre sorgo forrajero sobre la pastura.

\begin{tabular}{|c|c|c|c|c|c|c|c|c|c|}
\hline \multirow{2}{*}{$\%$} & \multicolumn{4}{|c|}{7,5 novillos/ha } & \multicolumn{4}{|c|}{10 novillos/ha } & \multirow{2}{*}{$\mathbf{P}$} \\
\hline & $T$ & EG & AA & GM & $\mathbf{T}$ & EG & AA & GM & \\
\hline \multicolumn{10}{|c|}{ OFRECIDO } \\
\hline FDA & 36,0 abc & $33,6^{b}$ & $35,7^{a b}$ & $37,1^{\mathrm{ab}}$ & $38,7^{a}$ & $34,8^{a b}$ & $35,9^{a b}$ & $34,4^{b}$ & * \\
\hline FDN & $61,8^{a b c}$ & $59,3^{c}$ & $62,2^{a b c}$ & $64,0^{a b}$ & $64,2^{a}$ & $61,7^{a b c}$ & $63,3^{a b}$ & $61,1^{b c}$ & * \\
\hline
\end{tabular}

Nota: medias con letras diferentes entre columnas son significativamente diferentes; ns = no significativo; * $=\mathrm{P}<0,05 ; \mathrm{T}=$ testigo sin suplementación; $\mathrm{EG}=$ suplementación con expeller de girasol; $\mathrm{AA}$ = suplementación con afrechillo de arroz; GM = suplementación con grano de maíz; FDA = fibra detergente ácido; FDN = fibra detergente neutro.

Cuadro 32. Efecto de la carga y suplementación de novillos sobre la composición botánica del sorgo forrajero.

\begin{tabular}{|c|c|c|c|c|c|c|c|c|c|}
\hline \multirow{2}{*}{$\%$} & \multicolumn{2}{|c|}{ Carga } & \multirow{2}{*}{$\mathbf{P}$} & \multicolumn{4}{|c|}{ Suplementación } & \multirow{2}{*}{$\mathbf{P}$} & \multirow{2}{*}{$\begin{array}{c}P \\
C * S\end{array}$} \\
\hline & Baja & Alta & & $\mathbf{T}$ & EG & AA & GM & & \\
\hline \multicolumn{10}{|c|}{ OFRECIDO } \\
\hline MS verde & 97,0 & 96,7 & ns & 95,4 & 96,2 & 98,3 & 97,5 & ns & ns \\
\hline Hoja & 39,5 & 39,7 & ns & 33,1 & 41,2 & 40,3 & 43,8 & ns & ns \\
\hline Tallo & 55,2 & 57,3 & ns & 62,6 & 54,5 & 55,8 & 52,2 & ns & ns \\
\hline Panoja & 5,3 & 2,9 & ns & 4,3 & 4,3 & 3,8 & 4,1 & ns & ns \\
\hline RS & 3,0 & 3,0 & ns & 4,6 & 3,8 & 1,7 & 2,5 & ns & ns \\
\hline \multicolumn{10}{|c|}{ REMANENTE } \\
\hline MS verde & 98,9 & 98,3 & ns & 98,9 & 99,3 & 98,0 & 98,1 & ns & ns \\
\hline Hoja & 21,0 & 16,7 & ns & 17,9 & 22,1 & 15,6 & 24,1 & ns & ns \\
\hline Tallo & 78,6 & 83,1 & ns & 86,8 & 77,5 & 82,3 & 75,9 & ns & ns \\
\hline Panoja & 0,4 & 0,2 & ns & 0,6 & 0,5 & 0,2 & 0 & ns & ns \\
\hline RS & 1,1 & 1,7 & ns & 1,1 & 0,7 & 2,0 & 1,9 & ns & ns \\
\hline
\end{tabular}

Nota: ns = no significativo; T = testigo sin suplementación; EG = suplementación con expeller de girasol; $\mathrm{AA}$ = suplementación con afrechillo de arroz; $\mathrm{GM}=$ suplementación con grano de maíz; $\mathrm{H}$ = hoja; $\mathrm{T}$ = tallo; $\mathrm{P}=$ panoja; $\mathrm{MS}=$ contenido de materia seca; $\mathrm{RS}=$ restos secos. 


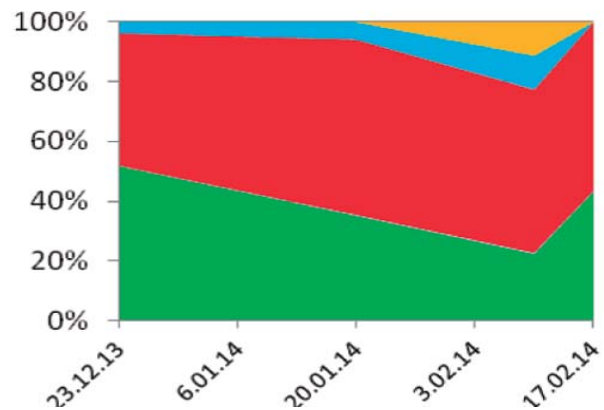

7,5 novillos/ha - $\mathrm{T}$

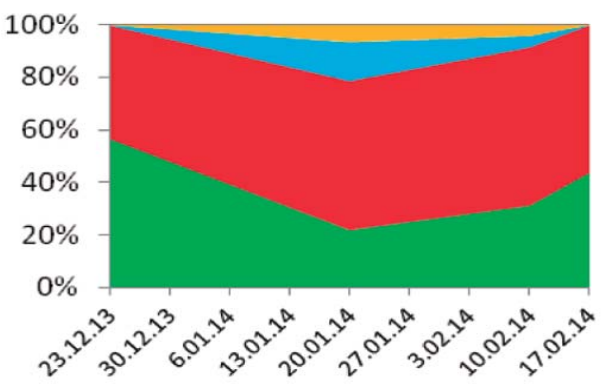

7,5 novillos/ha - AA

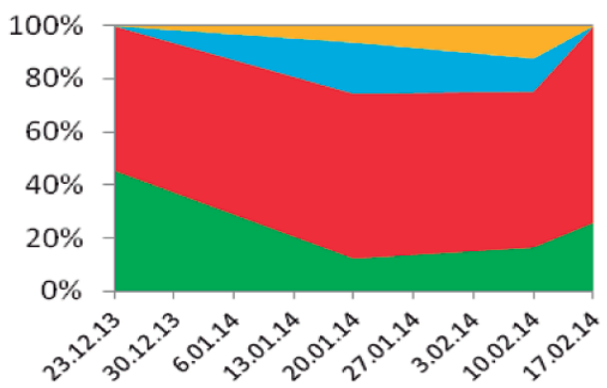

10 novillos/ha - T

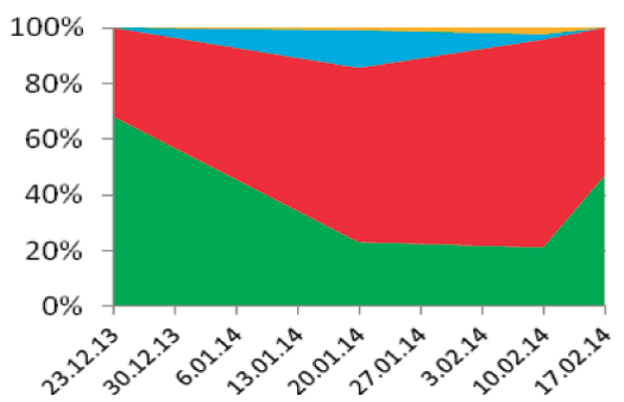

10 novillos/ha - AA

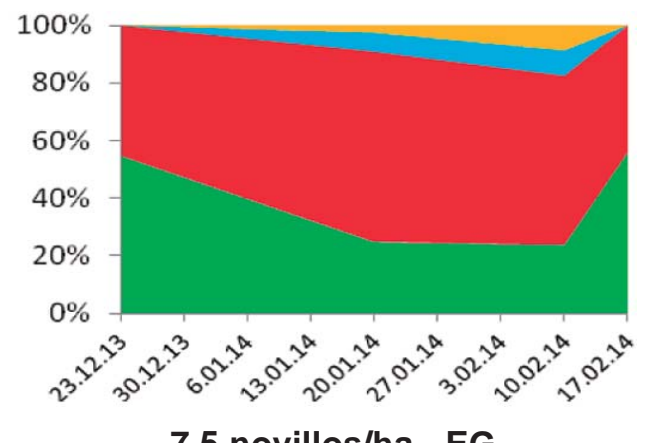

7,5 novillos/ha - EG

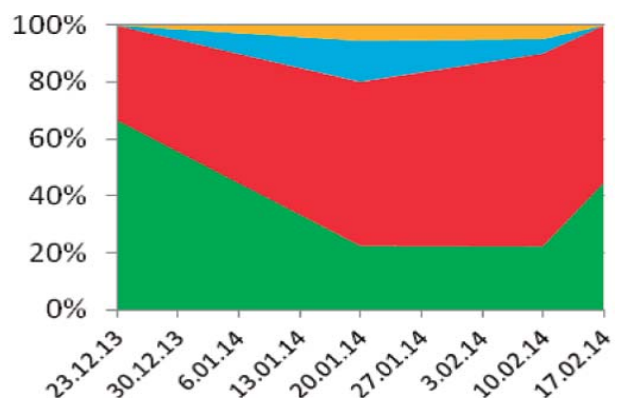

7,5 novillos/ha - GM

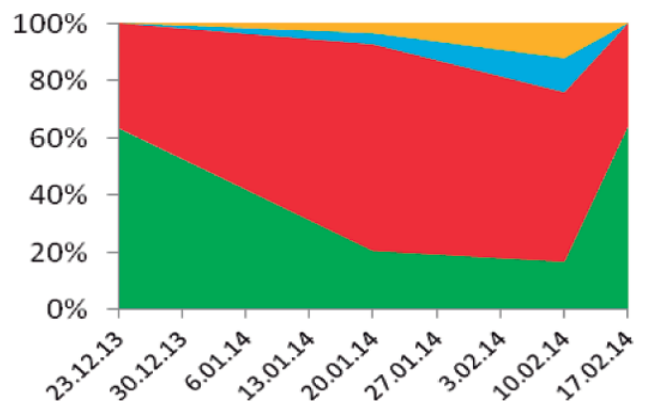

10 novillos/ha - EG

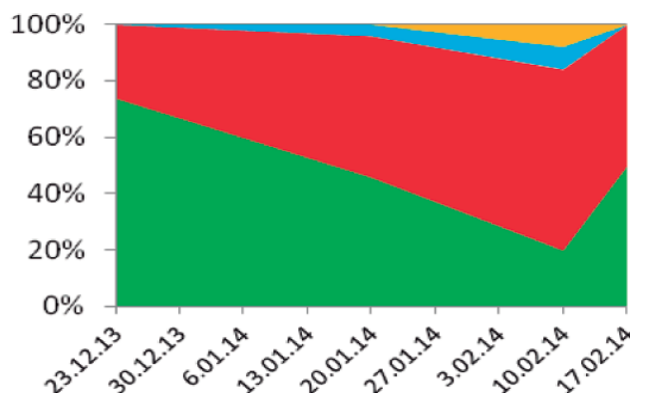

10 novillos/ha - GM

\section{$\square \mathrm{H} \square \mathrm{T} \square \mathrm{P} \square \mathrm{RS}$}

Nota: $\mathrm{T}$ = testigo sin suplementación; $\mathrm{EG}$ = suplementación con expeller de girasol; AA = suplementación con afrechillo de arroz; GM = suplementación con grano de maíz; $\mathrm{H}=$ hoja; $\mathrm{T}$ = tallo; $\mathrm{P}=$ panoja; $\mathrm{RS}=$ restos secos.

Figura 17. Evolución de la composición botánica del forraje ofrecido de sorgo forrajero con y sin suplementación y con dos cargas animales bajo pastoreo de novillos. 
contenido de fibra (FDA y FDN) del forraje ofrecido. Las interacciones significativas son ampliadas en el Cuadro 31.

El Cuadro 32 presenta los componentes de la composición botánica promedio del forraje ofrecido y remanente según carga animal y suplementación.

Coincidentemente con los resultados anteriormente presentados en esta publicación, la composición botánica no fue afectada $(P>0,05)$ por el tipo de suplementación, así como tampoco por la carga animal.

Rovira y Echeverría (2014) reportaron porcentajes de hoja del $49 \%, 26 \%$ y $21 \%$ para el primer, segundo y tercer pastoreo de un sorgo BMR con novillos de razas británicas. Si bien no se registraron diferencias estadísticamente significativas $(P<0,10)$ entre tratamientos, todos los porcentajes de hoja de la carga baja son mayores que los de la carga alta. Asimismo, lo inverso ocurre en la fracción tallo.

En la Figura 17 se presenta la evolución de la composición botánica del forraje ofrecido segun tratamiento para todo el período experimental.

En términos generales, la fracción hoja fue descendiendo desde el inicio del período pero aumentó abruptamente al final del período experimental en todos los tratamientos, producto de condiciones favorables que permitieron un rebrote del sorgo. De la misma manera, en general, se observa que la fracción panoja registra un aumento hacia la mitad del período, pero disminuyendo hacia el final del mismo. Esto podría estar asociado a un correcto manejo del pastoreo, que controló la fase reproductiva del cultivo, especialmente hacia el final del período experimental.

Al comparar el tratamiento T-carga baja con el T-carga alta, se observó que en el primer caso, el contenido de hoja inicial se ubicó apenas por encima del contenido final (diferencia menor del $10 \%$ ), mientras que en el segundo caso, el contenido inicial fue $20 \%$ mayor que el contenido de hoja final.

La Figura 18 presenta la evolución de la composición botánica del forraje rema- nente según tratamiento a lo largo del período experimental. La fracción tallo predominó en el forraje remanente en todos los tratamientos, a lo largo de todo el período. Asimismo, en todos los tratamientos, el contenido de hojas en el remanente tiende a ser insignificante al final del período experimental, con la única excepción del tratamiento GM-7,5 nov/ha, que presentó un $8 \%$ de contenido de hojas.

Se destaca que el contenido de panojas en todos los casos se mantuvo controlado a lo largo de todo el período, lo que es coherente con el correcto manejo reflejado en el forraje disponible.

\subsubsection{Resultados en comportamiento y producción animal}

Los resultados obtenidos en el comportamiento animal a partir de los dos factores evaluados (carga animal y uso de suplemento) se detallan en el Cuadro 33 y en la Figura 19, donde se presentan los resultados en conducta y tasa de bocado promedio para el período experimental, según tratamiento.

El pastoreo fue la actividad a la que los animales dedicaron más tiempo, lo cual es coincidente con los ensayos presentados anteriormente. El tiempo destinado al pastoreo no fue afectado por la carga animal empleada. Sin embargo, fue afectado significativamente $(P<0,05)$ por el factor suplementación, siendo los animales que no recibieron suplemento los que dedicaron mayor tiempo en esta actividad, en comparación a todos los animales suplementados.

Con respecto a la tasa de bocado, se registraron diferencias estadísticamente significativas $(P<0,05)$ entre las cargas utilizadas, siendo superiores las de la carga baja. La interacción entre ambos factores en la tasa de bocado, no determinó diferencias significativas $(P>0,05)$ en las variables estudiadas.

Los resultados del desempeño animal se presentan en el Cuadro 34, según carga animal y suplementación.Los resultados obtenidos en este estudio determinaron que el 

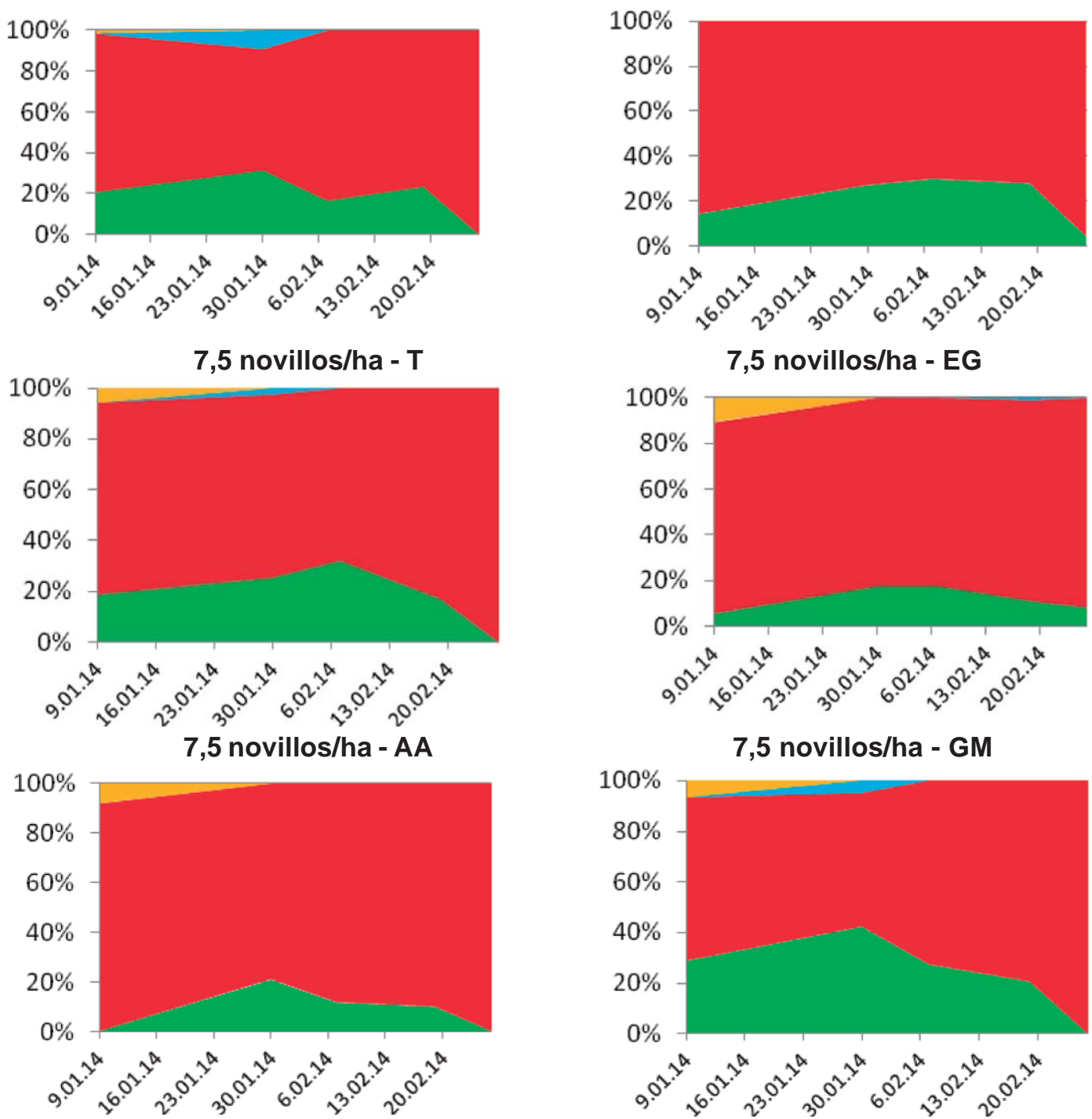

10 novillos/ha - T

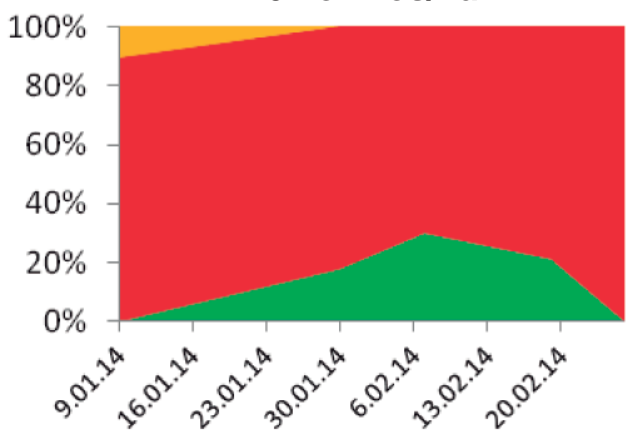

10 novillos/ha - AA

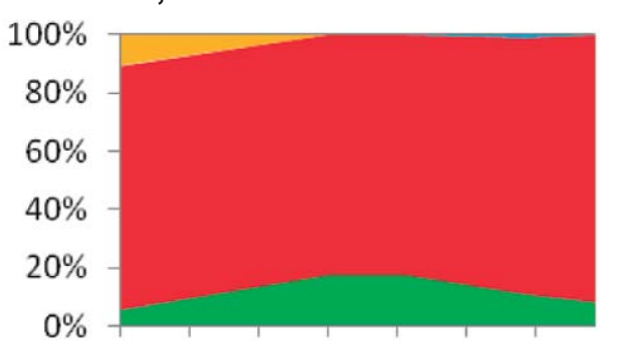

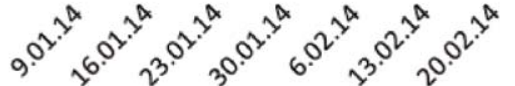

7,5 novillos/ha - GM

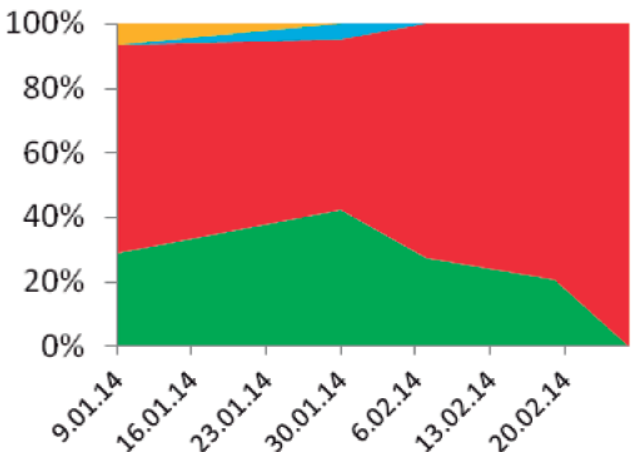

10 novillos/ha - EG

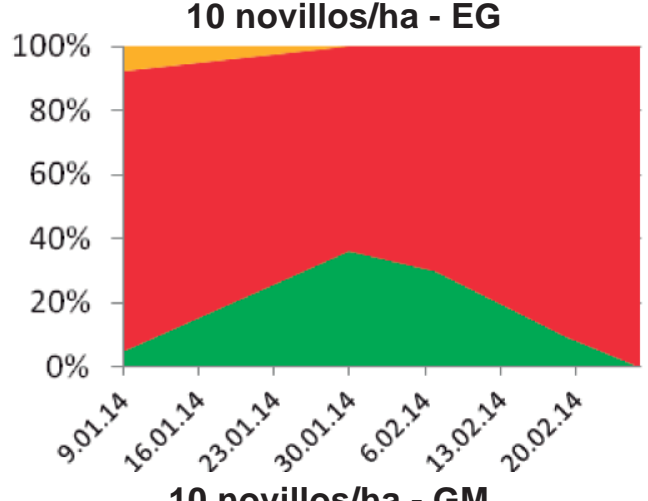

10 novillos/ha - GM

\section{$\square \mathrm{H} \square \mathrm{T} \square \mathrm{P} \square \mathrm{RS}$}

Nota: $\mathrm{T}$ = testigo sin suplementación; $\mathrm{EG}=$ suplementación con expeller de girasol; $\mathrm{AA}=$ suplementación con afrechillo de arroz; $\mathrm{GM}=$ suplementación con grano de maíz; $\mathrm{H}=$ hoja; $\mathrm{T}=$ tallo; $\mathrm{P}=$ panoja; $\mathrm{RS}=$ restos secos.

Figura 18. Evolución de la composición botánica del forraje remanente de sorgo forrajero con y sin suplementación y con dos cargas animales, bajo pastoreo de novillos. 
Cuadro 33. Efecto de la carga y la suplementación de novillos sobre la conducta animal (Año 1).

\begin{tabular}{|c|c|c|c|c|c|c|c|c|c|}
\hline \multirow{2}{*}{$\%$} & \multicolumn{4}{|c|}{7,5 novillos/ha } & \multicolumn{4}{|c|}{10 novillos/ha } & \multirow{2}{*}{$\mathbf{P}$} \\
\hline & $T$ & EG & $\mathrm{AA}$ & GM & $\mathbf{T}$ & EG & AA & GM & \\
\hline$P$ & 53,9 & 50,1 & ns & $59,5^{\text {a }}$ & $47,6^{b}$ & $50,6^{b}$ & $50,5^{b}$ & $\star \star$ & ns \\
\hline $\mathrm{R}$ & 13,6 & 12,7 & ns & 12,5 & 15,5 & 11,7 & 13,8 & ns & ns \\
\hline S & $3,8^{a}$ & $3,1^{b}$ & * & $0 \mathrm{~b}$ & $4,2^{a}$ & $4,8^{a}$ & $5,0^{a}$ & $\star \star$ & ns \\
\hline A & 4,5 & 4,1 & ns & 3,7 & 3,3 & 5,4 & 4,8 & ns & ns \\
\hline C & $2,1^{b}$ & $2,9^{a}$ & $*$ & 2,8 & 2,7 & 2,0 & 2,4 & ns & ns \\
\hline$D$ & $22,0^{b}$ & $27,1^{\mathrm{a}}$ & $\star \star$ & $21,5^{b}$ & $27,7^{\mathrm{a}}$ & $25,5^{a b}$ & $23,4^{b}$ & * & ns \\
\hline $\begin{array}{l}\text { Tasa de } \\
\text { bocado } \\
\text { (boc/min) }\end{array}$ & $16,5^{a}$ & $13,5^{b}$ & ** & 14,1 & 15,1 & 15,4 & 15,3 & ns & ns \\
\hline
\end{tabular}

Nota: medias con letras diferentes entre columnas son significativamente diferentes; ns = no significativo; * $=\mathrm{P}<0,05 ;{ }^{* *}=\mathrm{P}<0,01 ; \mathrm{T}=$ testigo; $\mathrm{EG}=$ expeller de girasol; $\mathrm{AA}=$ afrechillo de arroz; $\mathrm{GM}$ = grano de maíz. $\mathrm{P}=$ actividad de pastoreo; $\mathrm{R}=$ actividad de rumia; $\mathrm{S}=$ actividad de consumo de suplemento; $\mathrm{A}=$ actividad de consumo de agua; $C$ = actividad de caminata; $\mathrm{D}=$ descanso.

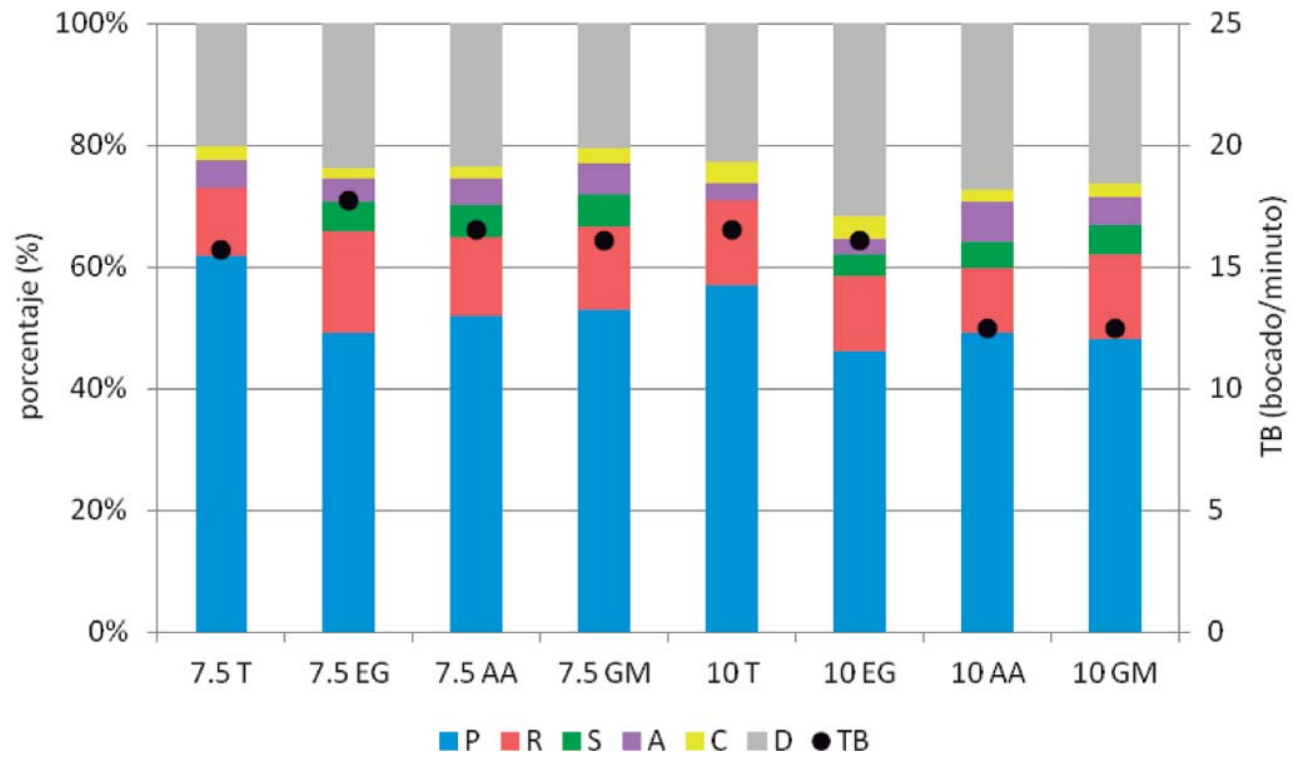

Nota: $\mathrm{T}$ = testigo sin suplementación; EG = suplementación con expeller de girasol; $A \mathrm{~A}=$ suplementación con afrechillo de arroz; $\mathrm{GM}$ = suplementación con grano de maíz; $\mathrm{P}=$ actividad de pastoreo; $\mathrm{R}$ = actividad de rumia; $\mathrm{S}=$ actividad de consumo de suplemento; $\mathrm{A}$ = actividad de consumo de agua; $\mathrm{C}=$ actividad de caminata; $\mathrm{D}=$ descanso.

Figura 19. Efecto de la carga animal y la suplementación sobre la conducta animal y tasa de bocado de novillos pastoreando sorgo forrajero.

factor carga animal tuviera un efecto significativo $(P<0,05)$ en el peso vivo lleno final, en donde los animales a una menor carga pesaron casi $17 \mathrm{~kg}$ más $(P<0,05)$ que aquellos que estuvieron a una carga de 10 novillos/ha. Las ganancias medias diarias, tanto para el peso vivo lleno como vacío, presenta- ron diferencias significativas $(P<0,05)$ para ambos factores evaluados. Todos los tratamientos suplementados tuvieron ganancias de peso vivo lleno significativamente superiores $(P<0,05)$ al tratamiento $T$. 


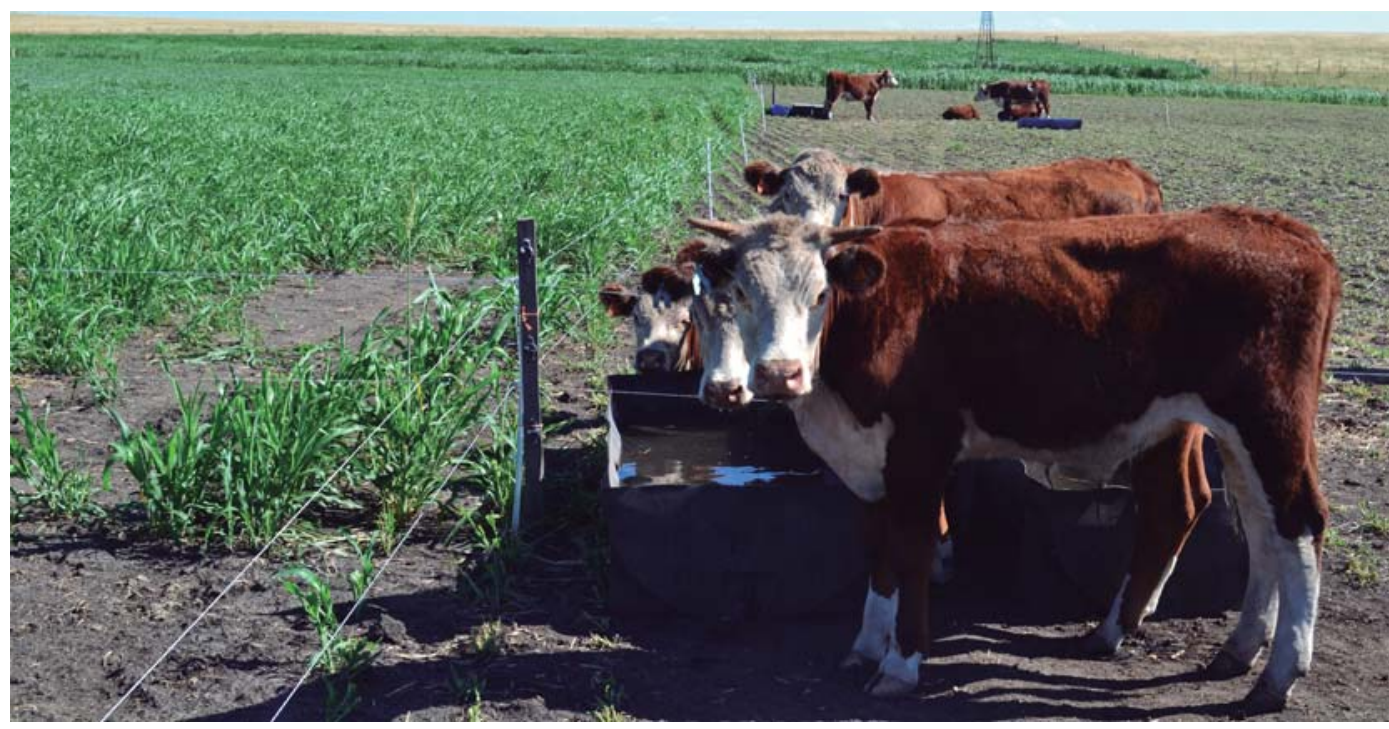

Cuadro 34. Efecto de la carga y la suplementación sobre el desempeño animal de novillos.

\begin{tabular}{|c|c|c|c|c|c|c|c|c|c|}
\hline \multirow{2}{*}{ Parámetro } & \multicolumn{2}{|c|}{ Carga } & \multirow{2}{*}{$\mathbf{P}$} & \multicolumn{4}{|c|}{ Suplementación } & \multirow{2}{*}{$\mathbf{P}$} & \multirow{2}{*}{$\begin{array}{l}P \\
C * S\end{array}$} \\
\hline & Baja & Alta & & $\mathbf{T}$ & EG & AA & GM & & \\
\hline PVLL i (kg) & 236,7 & 237,1 & ns & 237,4 & 236,8 & 236,8 & 237,0 & ns & ns \\
\hline PVLL $f(k g)$ & $279,5^{a}$ & $262,8^{b}$ & * & 260,9 & 272,8 & 274,7 & 276,3 & ns & ns \\
\hline PVV i (kg) & 221,6 & 222,0 & ns & 222,5 & 221,1 & 221,7 & 222,0 & ns & ns \\
\hline$P \vee V f(k g)$ & 255,8 & 245,1 & ns & 241,5 & 254,6 & 252,2 & 253,4 & ns & ns \\
\hline AA i $(\mathrm{cm})$ & 113,1 & 113,7 & ns & 113.8 & 113,1 & 113,1 & 113,7 & ns & ns \\
\hline $\operatorname{AA} \mathrm{f}(\mathrm{cm})$ & 118,3 & 117,7 & ns & 118,1 & 118,1 & 118,1 & 117,9 & ns & ns \\
\hline GPVLL (kg) & $0,453^{a}$ & $0,274^{b}$ & ** & $0,251^{b}$ & $0,382^{a}$ & $0,403^{a}$ & $0,418^{a}$ & $* *$ & ns \\
\hline GPVV (kg) & 0,359 a & $0,242^{b}$ & ** & $0,200^{b}$ & $0,352^{a}$ & $0,321 \mathrm{ab}$ & $0,330^{a}$ & ** & ns \\
\hline EC (kg S/kg PV) & - & - & - & - & 9,8 & 8,1 & 8,4 & ns & - \\
\hline $\begin{array}{l}\text { Producción } \\
\text { (kg PV/ha) }\end{array}$ & $319,5^{a}$ & $257,8^{\mathrm{b}}$ & ** & $193,0^{b}$ & $302,8^{a}$ & $322,0^{a}$ & $336,7^{a}$ & ** & ns \\
\hline
\end{tabular}

Nota: medias con letras diferentes entre columnas son significativamente diferentes; ns = no significativo; * $=\mathrm{P}<0,05 ;{ }^{*}=\mathrm{P}<0,01 ; \mathrm{T}=$ testigo sin suplementación; $\mathrm{EG}=$ suplementación con expeller de girasol; AA = suplementación con afrechillo de arroz; $G M=$ suplementación con grano de maíz; $i$ = inicial; $f$ = final; PVLL = peso vivo lleno; PVV = peso vivo vacío; $A A=$ altura de anca; $G P V$ = ganancia de peso vivo; $E C$ = eficiencia de conversión. (La EC fue calculada separadamente entre cargas; se presentan por separado los niveles de significancia).

Cuadro 35. Interacción entre la carga animal y suplementación sobre la eficiencia de conversión del suplemento de novillos pastoreando sorgo.

\begin{tabular}{|c|c|c|c|c|c|c|c|c|c|c|}
\hline & \multicolumn{4}{|c|}{7,5 novillos/ha } & \multirow{2}{*}{$\mathbf{P}$} & \multicolumn{4}{|c|}{10 novillos/ha } & \multirow{2}{*}{$\mathbf{P}$} \\
\hline & $T$ & EG & $\mathrm{AA}$ & GM & & $\mathrm{T}$ & EG & AA & GM & \\
\hline EC (kg S/kg PV) & - & 10,9 & 10.3 & 11,1 & ns & - & $8,6^{b}$ & $5,9^{a}$ & $5,8^{a}$ & *夫 \\
\hline
\end{tabular}

Nota: medias con letras diferentes entre columnas son significativamente diferentes; ns = no significativo; ** $=\mathrm{P}<0,01 ; \mathrm{T}=$ testigo sin suplementación; $\mathrm{EG}=$ suplementación con expeller de girasol; $A A$ = suplementación con afrechillo de arroz; GM = suplementación con grano de maíz. 
Con respecto a los valores de ganancias logrados, en términos generales, estos son los menores que se han registrado en los trabajos realizados en el marco de la presente publicación. Las referencias biliográficas consultadas que trabajaron con sorgos híbridos o BMR (Berlangeri, 2008; Vaz Martins et al., 2003; Rovira y Echeverría, 2013) siempre registraron valores superiores a los obtenidos en esta instancia.

Las EC del suplemento fueron comparadas dentro de cada carga animal evaluada. En este sentido, se registraron diferencias significativas $(P<0,05)$ en la carga de 10 novillos/ha a favor de los animales suplementados con AA y GM respecto a aquellos que recibieron EG, siendo menos favorables en esta última (Cuadro 35).

En la carga alta (10 novillos/ha) se registraron las mejores EC de todos los ensayos anteriormente presentados en esta publicación, e incluso al compararlas con los datos provenientes de la bibliografía nacional (Peruchena, 1999; Peruchena, 2003), que utilizaron animales de igual categoría que los de esta experiencia.

Pordomingo (2003), señala que, en términos generales, solamente con niveles de suplementación mayores al 0,75\% PV se manifiesta una mejora en la eficiencia; en esta experiencia se trabajó a razón del 0,50 \% PV de suplementación con cada tipo de alimento. Teniendo esto en cuenta, en combinación con el hecho que las EC de la carga baja fueron menores que las EC de la carga alta, puede decirse que la afirmación de este autor concuerda con los valores registrados en la carga baja, pero no así en el caso de la carga alta.

Al observar la producción total de carne por unidad de superficie (Cuadro 34), también se destaca que son de las menores registradas hasta el momento en esta publicación, en especial en el tratamiento $\mathrm{T}, \mathrm{y}$ que además fue significativamente menor a todas las demás producciones con inclusión de suplemento. En relación a la carga, la utilización de mayor cantidad de novillos por unidad de superficie determina menores producciones por hectárea.

La Figura 20 presenta la evolución del peso vivo según la carga animal y los animales suplementados (combinados) versus sin suplementar. Al observarse la evolución del peso vivo a lo largo del período experimental, se aprecia que, si bien los animales tu-

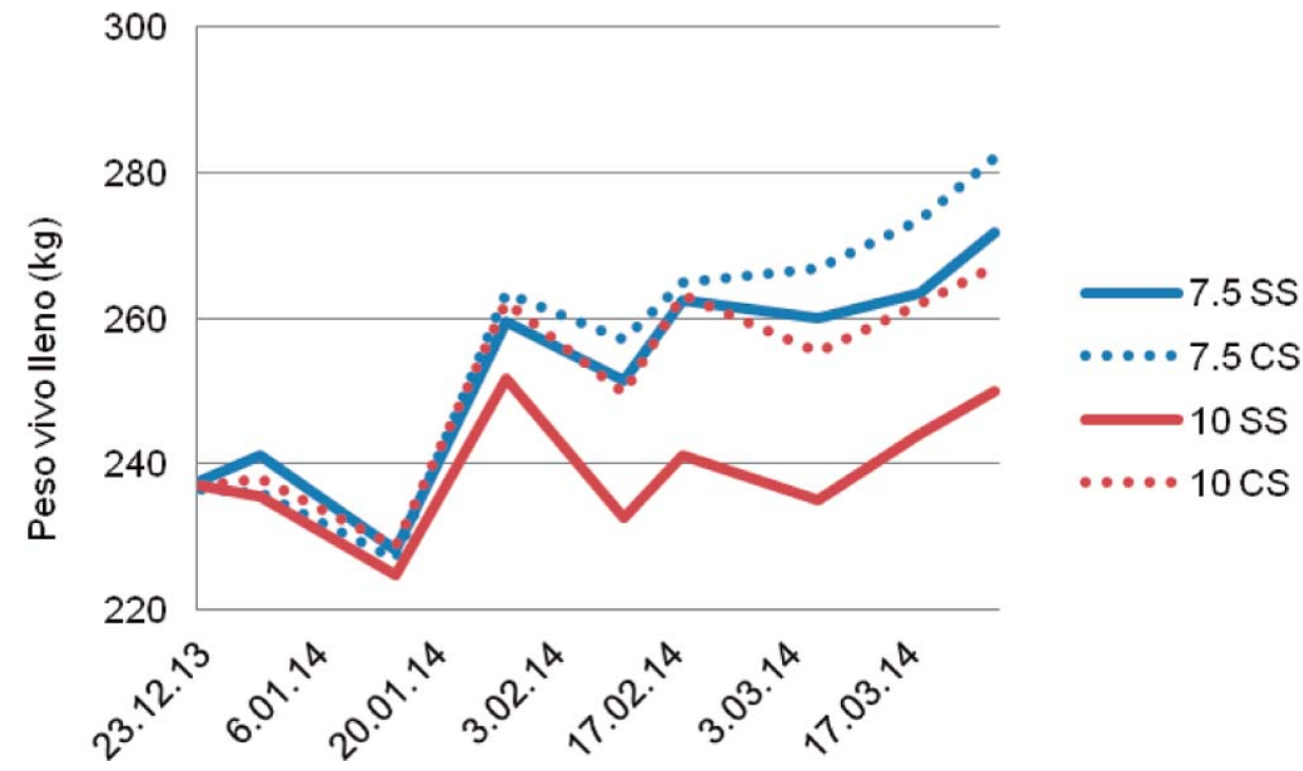

Nota: SS = sin suplementación; CS = con suplementación.

Figura 20. Evolución del peso vivo de los novillos según carga y suplementados vs. sin suplementar. 
vieron el correspondiente período previo de acostumbramiento al suplemento, recién a mediados de enero comenzó a observarse un aumento del peso vivo. Las pérdidas de peso hacia la mitad del período coincidieron con la disminución de la fracción hoja en ese momento y la predominancia de la fracción tallo.

En la Figura 20, se destaca consistentemente el menor desempeño animal de los novillos de la carga alta sin suplementación, lo cual es coincidente con los resultados finales de producción animal presentados en el Cuadro 34. También se destaca que la evolución del peso vivo de los animales correspondientes a la carga alta con suplementación se comportó de manera muy similar a la evolución registrada en los animales de la carga baja sin suplementación.

La Figura 21 presenta la evolución del peso vivo de los novillos durante el período experimental según la carga animal. En

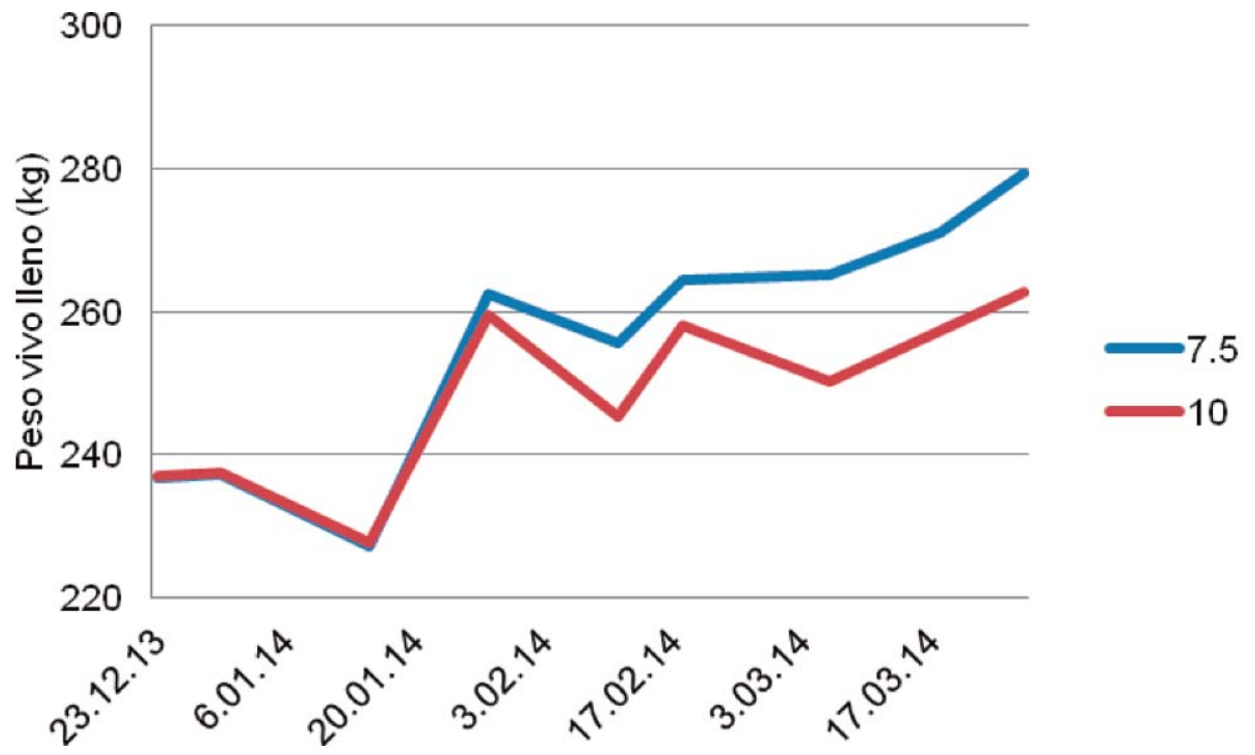

Figura 21. Evolución del peso vivo de los novillos según carga animal.

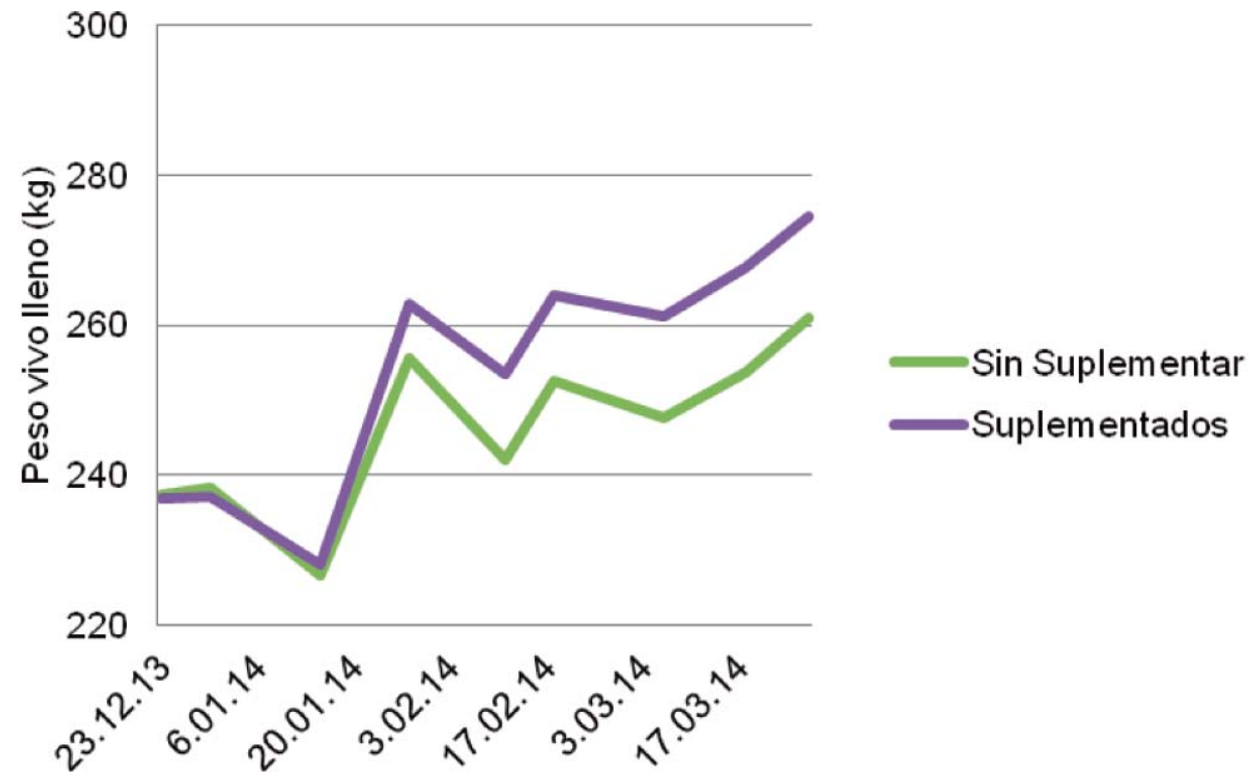

Figura 22. Evolución del peso vivo de los novillos comparando animales suplementados vs. sin suplementación. 
el primer tercio del período experimental, la evolución de los pesos vivos fue prácticamente la misma para ambas cargas animales, pero posteriormente se comienzan a visualizar diferencias por efecto de este factor. Como era de esperar, los animales manejados a una menor carga $(7,5$ novillos/ha) presentaron un mejor desempeño animal a partir de fines de enero, y las diferencias con la carga de 10 novillos/ha, se amplió aún más hacia el final del experimento.

La evolución del peso vivo de los animales evaluando el efecto de la suplementación se presenta en la Figura 22. El efecto de la suplementación en la evolución del peso vivo comenzó a visualizarse más tempranamente que el efecto de la carga animal. No obstante, la magnitud de las diferencias en la evolución del peso vivo entre animales suplementados y sin suplementar parecería mantenerse hasta el final del experimento.

Los parámetros de calidad de la canal según los factores de la carga animal y la suplementación se presentan en el Cuadro 36.

EI AOB no se vio afectado $(P>0,05)$ por la diferencia de carga o la inclusión y tipo de suplemento en el sistema, pero el engrasamiento de los animales a nivel de la $12^{\mathrm{a}}$ costilla fue mayor en animales que se encontraron a una menor carga y en los que fueron suplementados, en este último caso coincidiendo con Luzardo et al. (2014) si bien trabajando sobre pasturas sembradas. A nivel del cuadril (P8), la diferencia fue observada entre los animales T y EG con los AA y GM.

La proporción de animales a los que se les pudo efectuar la medición de grasa intramuscular (GIM) fue muy baja, por lo que no se presentan en este ensayo.

\subsubsection{Comentarios y conclusiones}

En esta experiencia, la cantidad y calidad del forraje ofrecido y remanente no presentó grandes diferencias entre los tratamientos evaluados, así como tampoco se registraron diferencias sustanciales al analizar cada factor por separado.

Las ganancias de PV estuvieron en un rango de 0,250 a 0,450 kg/an/día, con niveles de productividad por unidad de superficie de 190 a $330 \mathrm{~kg} \mathrm{PV/ha} \mathrm{en} \mathrm{un} \mathrm{período} \mathrm{con}$ una extensión de 95 días.

Los resultados del desempeño (individual y por unidad de superficie) obtenidos a cargas de 7,5 novillos/ha -con y sin suplementación- siguen la misma línea de los resultados obtenidos en las experiencias previas presentadas en esta publicación, con diferentes fuentes de suplementos, demostrando similares respuestas a los suplementos GM, AA y EG.

Cuadro 36. Efecto de la carga y suplementación sobre la calidad de la canal determinada in vivo.

\begin{tabular}{|c|c|c|c|c|c|c|c|c|c|}
\hline \multirow{2}{*}{$\%$} & \multicolumn{2}{|c|}{ Carga } & \multirow{2}{*}{$\mathbf{P}$} & \multicolumn{4}{|c|}{ Suplementación } & \multirow{2}{*}{$\mathbf{P}$} & \multirow{2}{*}{$\begin{array}{c}P \\
C * S\end{array}$} \\
\hline & Baja & Alta & & $T$ & EG & AA & GM & & \\
\hline AOB i $\left(\mathrm{cm}^{2}\right)$ & 29,9 & 29,5 & ns & 31,0 & 29,0 & 29,2 & 29,6 & ns & ns \\
\hline $\mathrm{AOB} f\left(\mathrm{~cm}^{2}\right)$ & 34,5 & 32,6 & ns & 33,2 & 34,0 & 33,8 & 33,3 & ns & ns \\
\hline EGS i (mm) & 1,9 & 1,8 & ns & 1,9 & 1,8 & 1,9 & 1,8 & ns & ns \\
\hline EGS $f(m m)$ & $2,0^{a}$ & $1,9^{b}$ & * & $1,9^{b}$ & $1,8^{b}$ & $2,1^{\mathrm{a}}$ & $2,0^{a b}$ & * & ns \\
\hline P8 i (mm) & 1,7 & 1,8 & ns & 1,8 & 1,7 & 1,8 & 1,7 & ns & ns \\
\hline$P 8 \mathrm{f}(\mathrm{mm})$ & 2,2 & 2,0 & ns & $2,0^{b}$ & $2,0^{b}$ & $2,4^{a}$ & $2,1^{a b}$ & * & ns \\
\hline
\end{tabular}

Nota: medias con letras diferentes entre columnas son significativamente diferentes; ns = no significativo * $=\mathrm{P}<0,05 ; \mathrm{T}=$ testigo sin suplementación; $\mathrm{EG}=$ suplementación con expeller de girasol; $\mathrm{AA}=$ suplementación con afrechillo de arroz; $G M=$ suplementación con grano de maíz; i = inicial; $f$ = final; $A O B=$ área ojo de bife; EGS = espesor grasa subcutáneas; P8 = espesor de grasa del cuadril. 
El explorar sistemas productivos con cargas de 10 novillos/ha sobre sorgos forrajeros sin suplementación no permitiría superar niveles de ganancias de peso de 0,300 kg/an/día e inclusive generaría un nivel de productividad por unidad de superficie inferior al de una carga de 7,5 novillos/ha, demostrándose así la limitación de la capacidad de carga de esta alternativa tecnológica. A esta carga extrema se observa el efecto positivo y aditivo de la inclusión de la suplementación, permitiendo que los niveles de ganancias de peso vivo alcancen valores cercanos a los 0,400 kg/an/día. En esta situación, las eficiencias de conversión alcanzan valores dentro del rango de 5,8 a 8,6 kg de suplemento por kg PV adicional obtenido. Aquí, la diferencia en el uso alternativo entre las diferentes opciones de suplementos probados, muestran ventajas a favor de GM y AA con relación a EG, explicado por la eficiencia de conversión lograda más que por las diferencias en las ganancias de peso obtenidas por las diferentes fuentes.

Los resultados de desempeño animal reafirman el uso del sorgo forrajero como una alternativa para mejorar la recría estival de novillos de sobreaño, demostrando su alta capacidad de carga pero sus restricciones para alcanzar ganancias altas, al menos en las condiciones productivas de suelos medios a profundos de Basalto.

\subsection{Evaluación del uso de diferentes suplementos sobre sorgo forrajero en la fisiología ruminal}

Este ensayo tuvo por objetivo principal evaluar el efecto del pastoreo de sorgo forrajero de alto rendimiento y el uso de diferentes suplementos en la fisiología ruminal de novillos fistulados.

\subsubsection{Materiales y métodos}

El período experimental comenzó el 9 de enero y finalizó el 18 de febrero (40 días).

Se llevó a cabo simultáneamente y en el marco del ensayo descripto en la sección 3.1.1. Materiales y métodos, por lo tanto, los tratamientos fueron los mismos que los pre- sentados en dicha sección (Año 1). Cada tratamiento contaba con 4 animales fistulados, con un peso vivo promedio inicial de $694 \mathrm{~kg}$.

Los animales fistulados fueron asignados a los diferentes tratamientos en 4 períodos experimentales de 10 días cada uno, dividiendo dicho período en dos subciclos ( 1 y 2 ) de 5 días cada uno en cada repetición de muestreo según el experimento de referencia ya mencionado. Cada novillo fistulado pasó por cada uno de los tratamientos ( $T, G M, A A$ y $E G$ ) y se encontraban pastoreando junto con los novillos del ensayo de referencia, durante todo el período experimental.

El último día de cada período del sub-ciclo 2 , se realizaron medidas cada 5 horas del líquido ruminal, comenzando a las 6:00 a.m. y culminando a las 9:00 p.m.

El muestreo del contenido ruminal se realizó una vez en cada estrategia de alimentación para cada animal fistulado. En cada día de muestreo se realizaban diferentes mediciones horarias, de tal forma que la primera se tomaba antes del suministro de suplemento y luego, los siguientes muestreos se realizaban a las 5, 10 y 15 horas posteriores del primer muestreo.

A cada una de las muestras de líquido ruminal se les medía el $\mathrm{pH}$ y posteriormente se conservaban a $-20^{\circ} \mathrm{C}$ con reactivo ácido (ácido sulfúrico al $5 \%$ ) hasta el momento de la determinación de la concentración de amonio en el Laboratorio de Nutrición Animal de INIA La Estanzuela.

\subsubsection{Diseño experimental}

El diseño experimental utilizado fue un diseño de cuadrado latino de $4 \times 4$. Se utilizó el procedimiento GLM del paquete estadístico SAS (SAS, 2013). Las medias se contrastaron con el test de LSmeans $(\mathrm{P}<$ $0,01)$.

\subsubsection{Resultados}

Los resultados de los parámetros descriptivos de la fisiología ruminal se presentan en el Cuadro 37. El pH ruminal es una variable de importancia clave, ya que sus cambios modifican el ambiente ruminal y repercuten 
Cuadro 37. Efecto de la suplementación de novillos pastoreando sorgo forrajero sobre la fisiología ruminal.

\begin{tabular}{|lccccc|}
\hline Parámetro & $\mathbf{T}$ & EG & AA & GM & P \\
\hline $\mathrm{pH}$ & 5,7 & 5,7 & 6,0 & 5,9 & $\mathrm{~ns}$ \\
$\mathrm{~N}-\mathrm{NH}_{3}\left(\mathrm{mgNH}_{3} / 100 \mathrm{ml}\right)$ & $31,5^{\mathrm{b}}$ & $46,3^{\mathrm{a}}$ & $29,5^{\mathrm{b}}$ & $34,6^{\mathrm{b}}$ & ${ }^{*}$ \\
\hline
\end{tabular}

Nota: medias con letras diferentes entre columnas son significativamente diferentes; ns = no significativo; ${ }^{\star *}=\mathrm{P}<0,01 ; \mathrm{T}=$ testigo sin suplementación; $\mathrm{EG}=$ suplementación con expeller de girasol; $A A$ = suplementación con afrechillo de arroz; $G M=$ suplementación con grano de maíz.

sobre su dinámica. Los valores de $\mathrm{pH}$ en el rumen se encuentran influenciados por varios factores, como ser el tipo de alimentación y el tiempo de ingestión. Normalmente se encuentran en valores de entre 5,5 y 6,9 (Relling y Mattioli, 2003).

En el presente estudio, las diferentes estrategias nutricionales no presentaron un efecto significativo $(\mathrm{P}>0,05)$ en el $\mathrm{pH}$ ruminal, encontrándose los mismos dentro de los valores normales mencionados (Relling y Mattioli, 2003).

Los niveles normales de concentración de amoníaco en el rumen varían según diferentes autores, oscilando entre 2 y $50 \mathrm{mg}$ por $100 \mathrm{ml}$, dependiendo de la ración y del tiempo transcurrido desde la ingesta. Este compuesto es el principal nutriente nitrogenado para las bacterias del rumen (Garriz y López, 2002). Si la concentración del N amoniacal es baja, desciende la efectividad del crecimiento microbiano, por lo cual, también la síntesis de proteína microbiana, la que re- presenta el $40 \%$ del $\mathrm{N}$ no amoniacal que se absorbe a nivel del intestino (Church, 1988).

En el estudio, el nitrógeno amoniacal (N$\left.\mathrm{NH}_{3}\right)$ fue afectado $(\mathrm{P}<0,01)$ por la dieta. Los niveles de $\mathrm{N}-\mathrm{NH}_{3}$ fueron significativamente mayores $(P<0,05)$ en el caso de la suplementación con EG que en los otros tres tratamientos. Esto tendría su lógica ya que el EG es un suplemento proteico, por lo cual es esperable que el nivel de $\mathrm{N}^{-\mathrm{NH}_{3}}$ aumentara más que en los casos de la suplementación energética (GM), energética-proteica (AA) o directamente sin suplementación (T) (Church, 1988).

Cuanto menor sea el $\mathrm{pH}$ en el rumen, mayor será la captación de amoníaco en forma de ion $\left(\mathrm{NH}_{4}\right)$. Además, la absorción del amoníaco desde el rumen depende del $\mathrm{N}$ amoniacal y del pH (Church, 1988), con lo cual ambos parámetros están muy relacionados. Sin embargo, en este estudio, el pH no fue afectado significativamente pero sí lo fue el nitrógeno amoniacal.

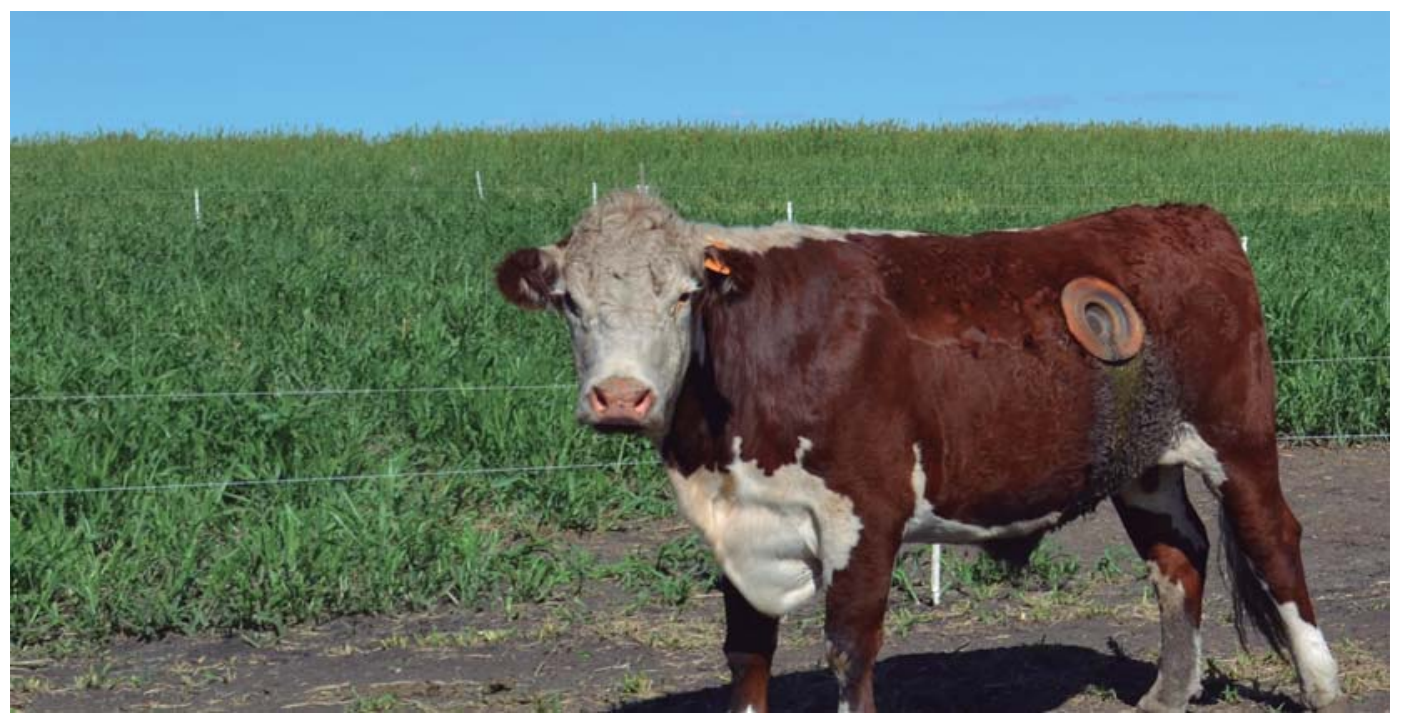




\subsubsection{Comentarios y conclusiones}

La suplementación con distintas fuentes no varió el $\mathrm{pH}$ ruminal de los animales, pero sí afectó el contenido de amonio en sangre. Esto significa que los animales suplementados con un concentrado proteico (EG) lograron aumentar la concentración de $\mathrm{N}$ amoniacal en rumen, posiblemente favoreciendo la síntesis de proteína microbiana, de muy elevada calidad biológica para el animal. Esto cobra especial importancia en animales jóvenes que se encuentran en etapa de recría.

A pesar de estas diferencias, si se considera la performance individual y grupal de los animales que estaban bajo el mismo tratamiento que los de este ensayo (Cuadros 20 y 21), se observa que no se registraron diferencias significativas en ningún parámetro, salvo en la EC, aunque el tratamiento con suplemento proteico (EG) no fue quien presentó mejor eficiencia.

\section{RESUMEN Y DISCUSIÓN CONJUNTA DE LA INFORMACIÓN PRESENTADA}

A partir de la información presentada previamente, se sintetizan y sistematizan una serie de consideraciones y reflexiones finales en relación el engorde de novillos sobre verdeos estivales en la región de Basalto.

\subsection{Base forrajera: sorgo híbrido vs. sudangrás}

En términos generales, como fuera indicado en la revisión bibliográfica, los sorgos híbridos han representado una alternativa mejorada al sudangrás para mejorar la productividad animal.

En las experiencias presentadas en esta publicación, el sorgo forrajero tipo BMR resultó siempre superior al sudangrás, especialmente en términos de la ganancia de peso y la producción de peso vivo por unidad de superficie (Cuadros 7 y 13). Las diferencias en términos de la conducta animal y deposición de tejidos en el animal fueron escasas o nulas (Figuras 3 y 7 ; Cuadros 8 y 14).
Estas diferencias se deben principalmente a la mayor disponibilidad de forraje generado por el sorgo híbrido en comparación con el sudangrás (Cuadros 3 y 9) y en menor medida a las diferencias observadas en los parámetros del valor nutritivo analizados en estos estudios (Cuadros 4 y 10).

La incorporación de la suplementación cuando se compararon estos dos tipos de sorgos no influyó en la producción vegetal (Cuadros 3 y 9 ) o en su valor nutritivo (Cuadros 4 y 10).

\subsection{Manejo del pastoreo y comportamiento animal}

Al examinar lo ocurrido con la composición botánica, se observa que en todos los ensayos la proporción de panoja en el forraje, tanto de sudangrás como de sorgo, siempre fue mínima. Esto estaría indicando un correcto manejo del pastoreo (rotativo) aplicado con franjas de pastoreo por 7 días con descansos de 21 días entre pastoreos, permitiendo así que la fracción vegetativa del forraje siempre predominara por sobre la reproductiva y/o restos secos. Se puede destacar entonces que el manejo del pastoreo propuesto a las cargas consideradas fue correcto y puede recomendarse para las condiciones manejadas en estas experiencias. De cualquier manera, cuando sea necesario, el uso estratégico de una rotativa puede controlar rápidamente potenciales excesos de forraje y sus implicancias positivas en la mejora del valor nutritivo del forraje y la producción animal.

En cuanto al comportamiento animal, se concluye que el pastoreo tendió a ser siempre la actividad a la que los animales le dedicaron más tiempo, independientemente del año de evaluación y del tipo de ensayo. En los dos primeros ensayos se observó que los tratamientos suplementados presentaron significativamente menor tiempo dedicado al pastoreo, en contraste con los animales no suplementados. Posiblemente la adición de nutrientes mediante el suplemento permitió mejorar la dieta global (cantidad y calidad) de los animales, lo cual repercutió en que los animales requirieran de un menor aporte 


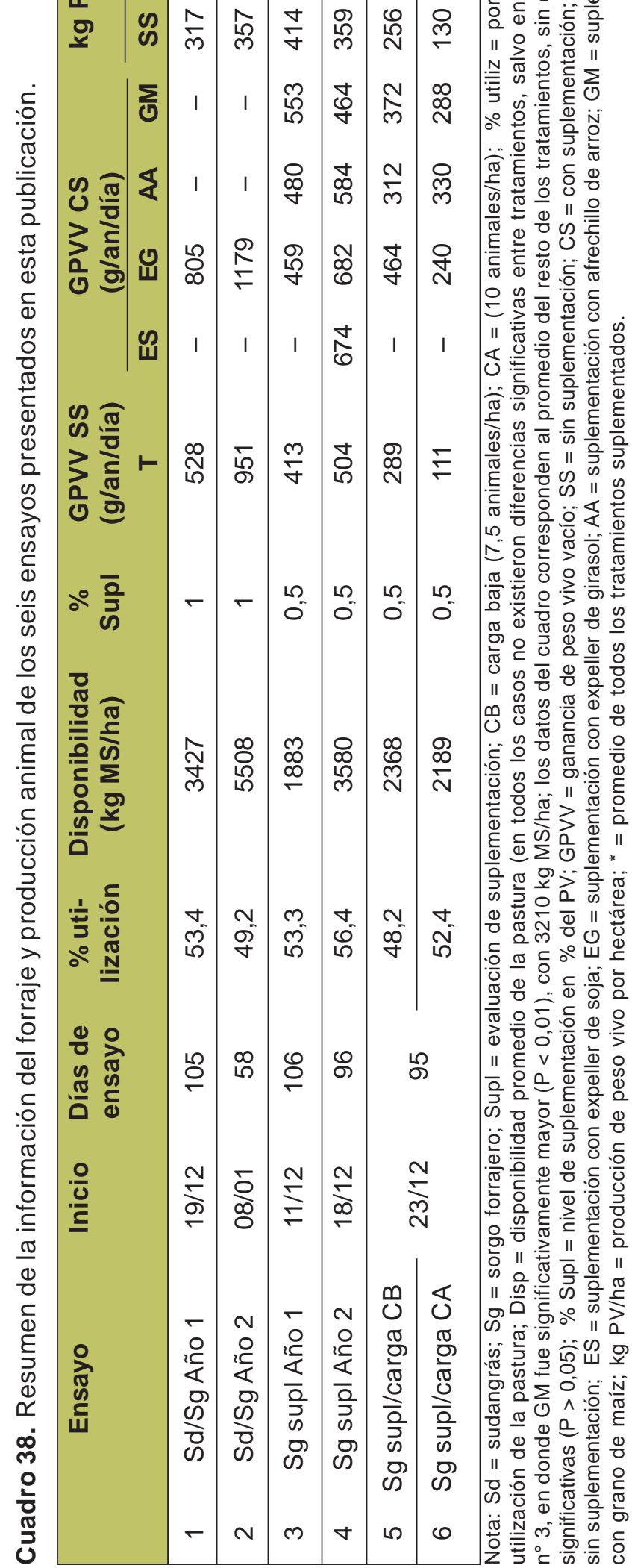


relativo desde el forraje, en comparación con aquellos provenientes del suplemento. Sin embargo, en los demás ensayos esta tendencia no fue tan clara. Los niveles de suplementación utilizados fueron bajos $(0,5$ a $1,0 \%$ del PV) y esto podría explicar los efectos menores de la suplementación sobre los parámetros de la conducta animal.

\subsection{Producción forrajera y producción animal}

En el Cuadro 38 se presenta un resumen de los principales resultados en relación al forraje y producción animal de todos los ensayos considerados en esta publicación.

Como se puede apreciar, salvo en el caso del Ensayo 2 que tuvo que ser finalizado tempranamente (duración total de aproximadamente 2 meses) debido a intensas precipitaciones registradas durante los meses de verano que impidieron un correcto seguimiento del mismo, los ensayos tuvieron un rango de duración de 95 a 106 días. Este período coincide -en términos generales- con el tiempo de utilización del cultivo en condiciones comerciales, lo que facilita la interpretación y uso de la información en el contexto de estos sistemas productivos. Es importante destacar que la información obtenida fue generada sobre suelos medios a profundos del Basalto, donde pueden ubicarse estas propuestas tecnológicas para mejorar la producción de forraje y su efecto positivo en la producción animal. El modelo conceptual subyacente se presenta en la Figura 23 (Montossi et al., 2014).

En las áreas "verdes" representadas en la Figura 23 se pueden implementar esquemas forrajeros mejorados, donde el cultivo de sorgo puede ser parte de una rotación de cultivos forrajeros de verano e invierno (avenas y raigrases) y pasturas cultivadas con diferentes mezclas de gramíneas y leguminosas. La rotación forrajera seleccionada no solo tendrá en cuenta la potencialidad de los suelos para la optimización de la productividad vegetal y animal, si no por sobre todo,

\section{La Intensificación de la Ganadería Extensiva El ejemplo del Basalto}
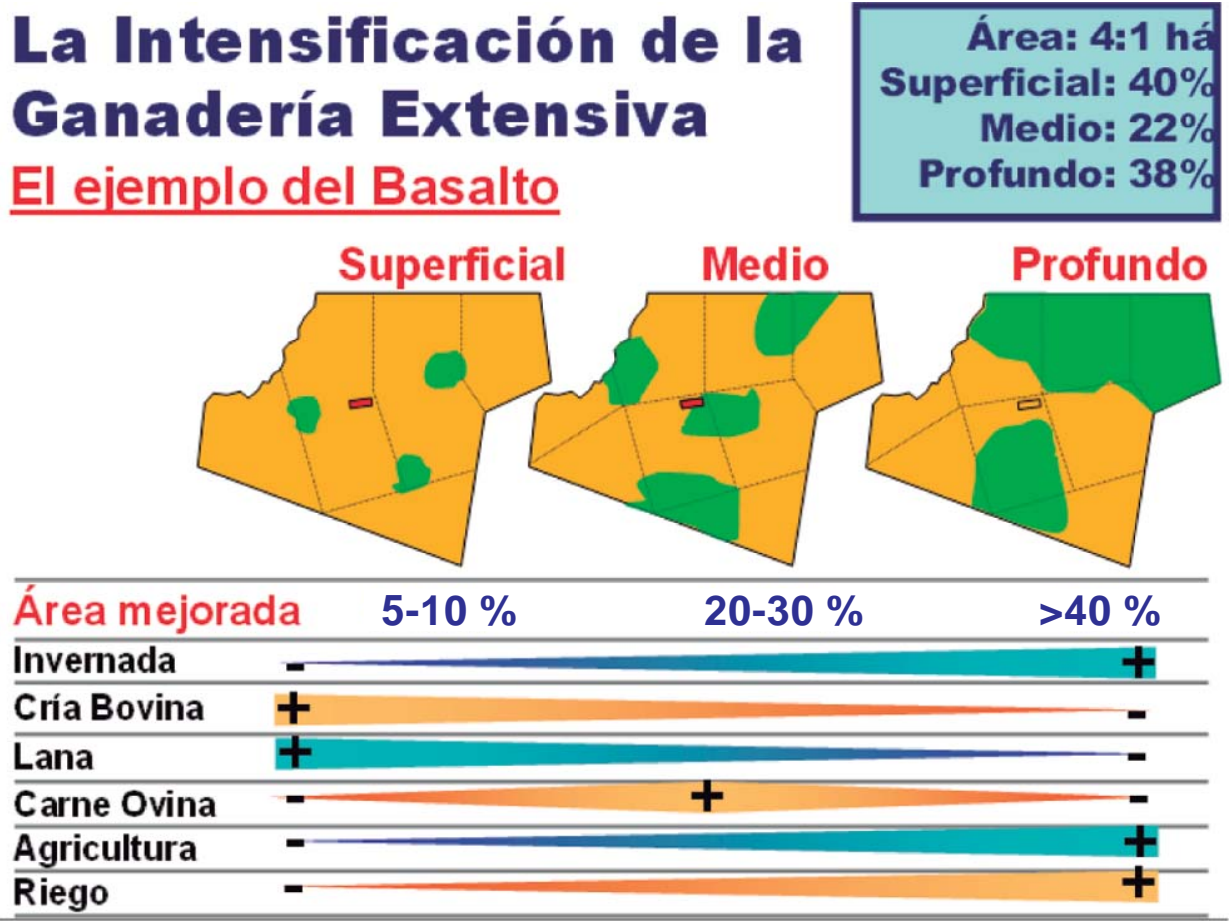

Nota: Áreas en naranja = suelos superficiales; áreas verdes = suelos medios y profundos.

Figura 23. Representación esquemática y conceptual de la especialización productiva ovina y bovina, combinada con otros rubros agrícolas, de acuerdo a la aptitud productiva de los diferentes suelos del Basalto (Montossi et al., 2014). 
la maximización del margen bruto de la rotación planteada como un todo. Por otra parte, además del efecto de las condiciones agroecológicas sobre la producción de forraje de los sorgos utilizados sobre los suelos mencionados, es importante señalar que es imprescindible seguir las recomendaciones agronómicas (ej. densidad de siembra, control de malezas, fertilización) para el cultivo de sorgo forrajero en la búsqueda de favorecer su producción forrajera y animal.

Las utilizaciones de forraje oscilaron entre 45,5 y $46,9 \%$ (Cuadros 3 y 9 ) en el caso del sudangrás, y entre un máximo de 58,3\% (Cuadro 22) y un mínimo de 45,0 \% (Cuadro 28), para el caso del sorgo forrajero utilizando una carga de 7,5 animales/ha. Al aumentar la carga a 10 animales/ha, las utilizaciones del sorgo se ubicaron en el entorno de 50,6 \% (Cuadro 28). Estos valores son cercanos a los reportados por la bibliografía nacional para sudangrás: 53,5\% (Rovira, 2002), 44-72 \% (Gabard y Russi, 2005), 36$45 \%$ (Esquivel et al., 2006) y Vaz Martins et al. (2003) 42-49 \%; y para sorgo forrajero: Vaz Martins et al. (2003) 45-53\% y Berlangeri (2008) 48-58 \%.

Las variaciones en el tipo de sorgo usado, el nivel de suplementación (0,5-1,0\% del $P V)$ y el tipo de suplemento empleado no tuvieron una clara implicancia en el nivel de utilización logrado.

La morfología y fisiología de los sorgos de pastoreo no permite una alta utilización de pastoreo como ocurre con otras especies de gramíneas, templadas o sub-tropicales. Por otro lado, el uso de mayores cargas animales por unidad de superficie como instrumento de mejora de la utilización de forraje, tendría implicancias negativas en la producción animal.

Al analizar la cantidad de forraje disponible promedio de cada ensayo, en ningún caso se registraron diferencias significativas entre los tratamientos, con la única excepción del Ensayo 3 y solamente distinguiéndose el tratamiento GM por su mayor forraje disponible. El tipo y nivel de suplementación empleados en estos ensayos demuestra que no tienen implicancias mayores en la producción de forraje en los sorgos utilizados.
Se observó, en términos generales, que a una misma carga animal (7,5 novillos/ha), el pasar de suplementar de una tasa del $1 \%$ PV (Ensayos 1 y 2) a 0,5 \% PV (Ensayos 3 al 5), significó que las ganancias individuales se vieran disminuidas. Si solo se toma en cuenta el Ensayo 1 -con un período comparable a los demás ensayos- se observa que los tratamientos suplementados presentan mejores desempeños individuales $(0,321$ 0,805 kg/an/día; Cuadros 34 y 7) y producción por hectárea (343-577 kg PV/ha; Cuadros 25 y 7) que los no suplementados (0,200-0,528 kg/an/día; Cuadros 34 y 7, y 193-414 kg PV/ha; Cuadros 34 y 20), independientemente de la base forrajera considerada. Estos aumentos de ganancia con respecto a T tuvieron una magnitud de 52-60 \%, mientras que la producción de $\mathrm{kg} P V / \mathrm{ha}$ de lotes suplementados fue entre un $40 \%$ y un $78 \%$ superior a los lotes no suplementados.

Según los estudios liderados por Beretta et al. (2008) sobre praderas artificiales durante el verano, para obtener una respuesta a la suplementación es necesario manejar el ganado con una asignación de forraje (AF) restringida (3 \% PV). En este caso, se lograría una eficiencia de conversión de 6:1; mientras que asignaciones de forraje del orden del 9-12\% no tendrían respuesta a la suplementación en las condiciones estudiadas. En los ensayos presentados en esta publicación, las asignaciones de forraje oscilaron entre 3,3 y $5,5 \%$ cada $100 \mathrm{~kg}$ de PV por lo que se estaría algo por encima del límite que estos autores consideran para una AF restringida. A ello se debería agregar el componente de valor nutricional del forraje ofrecido del sorgo forraje, el cual en general es menor al de las praderas cultivadas en el verano (Carámbula, 2007b). Como se puede observar en los Cuadros 20, 25 y 34, la EC fue muy variable en función del año de evaluación y del suplemento evaluado. Es el caso de suplemento GM con resultados variables entre experimentos (Cuadros 20 y 34).

Por otro lado, en términos cuantitativos, se observa por ejemplo que un mismo suplemento $(E G)$ presentó una diferencia máxima de 43,2 puntos (Cuadros 20 y 34) entre un año y otro. En definitiva, las EC fueron su- 
mamente variables y por lo tanto pueden considerarse difíciles de predecir con precisión a partir de la información generada en estas experiencias.

En cuanto a la carga óptima para este tipo de cultivos, los Ensayos 5 y 6 demuestran que al aumentar la carga de 7,5 a 10 novillos/ha sin suplementación, tanto el desempeño individual como la producción por unidad de superficie, se vieron afectados negativamente. En definitiva, en base a los resultados obtenidos en este ensayo, el pasar de una carga de 7,5 a 10 novillos/ha sin el uso de suplemento, significaría afectar negativamente tanto la ganancia individual como la producción por hectárea.

El desempeño animal individual sobre verdeos de verano con una carga de 7,5 animales/ha sin suplementación, logró un máximo de 0,951 kg/an/día, lo cual se logró en un ensayo que tuvo una duración de 58 días en total (Ensayo 2). Para el resto de los ensayos, con una duración aproximadamente equivalente al período normal de utilización del cultivo, el rango osciló entre un máximo de 0,528 (Cuadro 7) kg/an/día y un mínimo de $0,200 \mathrm{~kg} / \mathrm{an} /$ día (Cuadro 34).

Al comparar estas ganancias con las reportadas por Vaz Martins et al. (2003), quienes trabajaron por períodos superiores a los 100 días con $60 \mathrm{~cm}$ de altura al ingreso a las parcelas de sudangrás y sorgo forrajero, lograron una ganancia promedio de 0,906 kg/an/día. De cualquier manera, estos autores trabajaron con cargas de 3,0-3,5 UG/ha y los animales comenzaron con pesos cercanos a los $190 \mathrm{~kg}$ PV, finalizando con 270-290 kg PV, es decir, se trataba de un pastoreo mucho menos intenso en dotación animal y se utilizaron animales más jóvenes y por lo tanto más eficientes biológicamente.

Por otro lado, Rovira (2002) trabajando con sudangrás y animales similares a los de estos ensayos ( $P V$ inicial $=400 \mathrm{~kg}$ ), con utilizaciones de forraje también similares $(53,5 \%)$ -pero con una carga menor, correspondiente a 5,5 UG/ha- y solamente por un período de 53 días, lograron ganancias promedio de $0,451 \mathrm{~kg} / \mathrm{an} / \mathrm{día}$. En este caso, es la dotación animal utilizada la que podría explicar las buenas ganancias registradas, que sin embargo, se encuentran dentro del rango reportado para los ensayos de la presente publicación, que duraron aproximadamente 100 días.

Por otra parte, al analizar el factor suplementación sin considerar la carga manejada, los resultados indican que sería conveniente suplementar sobre esta base forrajera, ya que se obtienen mejores ganancias de peso vivo y producciones totales por unidad de superficie.

Al contrastar las cargas evaluadas en las mismas condiciones (Ensayos 5 y 6 ) en relación al efecto de la suplementación, se observa que los animales suplementados utilizando la carga baja presentaron performances individuales $34 \%$ mayores en promedio que los animales suplementados de la carga alta. Al contrastar estos dos lotes en producción total, la carga baja presentó una producción $15 \%$ superior a la carga alta. A la razón de suplementación considerada $(0,5 \% \mathrm{PV})$, tanto la producción individual como producción por hectárea se vieron resentidas por utilizar una carga de 10 noviIlos/ha a igualdad de condiciones ambientales, por lo que no se verificó ninguna ventaja por utilizar una carga mayor.

Sobre las bases forrajeras consideradas, en términos del desempeño animal, no se observó una clara superioridad por el uso de un determinado suplemento versus otra opción, donde se compararon distintas clases de suplementos: proteicos (ES y EG), energético-proteicos (AA) y energéticos (GM). Inclusive no se lograron diferencias en algunos casos en la respuesta individual entre los diferentes suplementos y el tratamiento testigo sin suplementación. Sin embargo, cuando los efectos de la suplementación se evalúan a nivel de la producción por unidad de superficie, esta tecnología fue consistentemente superior a los tratamientos donde los animales no recibieron suplemento.

Del punto de vista de la comparación entre las diferentes fuentes de suplementos en la respuesta animal, se presentaron solo dos ocasiones donde existieron diferencias significativas entre tratamientos, que fueron los casos de los Ensayos 4 y 5 . En el primer 
caso, solamente se puede afirmar que la performance individual en los animales suplementados con GM (energético) fue inferior a la de los novillos suplementados con ES y EG (proteicos), mientras que los animales suplementados con AA presentaron valores intermedios (Cuadro 25). En el Ensayo 5 se observó que la performance individual de los animales suplementados con AA (suplemento energético-proteico) no fue estadísticamente diferente a EG y GM, pero tampoco lo fue del testigo ( $T$ ) (Cuadro 34). Por lo tanto, para las condiciones experimentales que se presentaron en estos ensayos, el uso de las diferentes fuentes de suplementos no tendrían implicancias consistentes en la producción animal, pero sí podrían tenerlas en la respuesta económica, más aún si se incluye la eficiencia de conversión de suplemento en peso vivo.

\section{BIBLIOGRAFÍA}

BERLANGERI, S. 2008. Efecto del manejo y el material genético en la productividad de sorgo forrajero bajo pastoreo. Tesis Ingeniero Agrónomo. Montevideo, Uruguay. Facultad de Agronomía. 189 p.

BERETTA, V.; SIMEONE, A. ELIZALDE, J. 2008. Manejo de animales en engorde durante el verano. En: Una década de investigación para una ganadería más eficiente: Décima jornada anual de la Unidad de Producción Intensiva de Carne. Montevideo: Facultad de Agronomía. E.E.M.A.C. p. 29-31.

CARÁMBULA, M. 2007a. Verdeos de verano. Montevideo: Hemisferio Sur. 226 p.

CARÁMBULA, M. 2007b. Pasturas y forrajes: potenciales y alternativas para producir forraje. Montevideo: Hemisferio Sur. v. 1. $357 \mathrm{p}$.

CHURCH, C. 1988. El rumiante: fisiología digestiva y nutrición. Zaragoza: Acribia. 641 p.

COSGROVE, G.; EDWARDS, G. 2007. Control of grazing intake. En: Rattray, P.V.; Brookes, I.M.; Nicol, A.M. (eds.). Pasture and supplements for grazing animals. Hamilton: New Zealand Society of Animal Production. p. 61-80. (Occasional publication; 14).
CONTRERAS-GOVEA, F.; MARSALIS, M.; LAURIAULT, L.; BEAN, B. 2010. Forage sorghum nutritive value: A review. Forage and Grazinglands, 8(1).

ESQUIVEL, J.; VELAZCO, J.; ROVIRA, P. 2006. Efecto del acceso a sombra artificial en la ganancia de peso, estrés y conducta de novillos pastoreando sudangrás durante el verano. En: Jornada Anual Unidad Experimental Palo a Pique. INIA Treinta y Tres. Montevideo: INIA. p 2236. (Serie Actividades de Difusión; 511).

ELIZALDE, J. 2003. Suplementación en condiciones de pastoreo. [En línea]. Consultado 19 ene. 2017. Disponible en http:// www.produccion-animal.com.ar/ informacion_tecnica/suplementacion/ 13-suplementacion_en_condiciones_de_pastoreo.pdf

FERNÁNDEZ MAYER, A.; STUART, R.; CHONGO, B.; MARTIN, P. 2011. Ceba pastoril con sorgos nervadura marrón o BMR (Brown Middle Rib) como forraje fresco. Revista Cubana de Ciencia Agrícola, 45 (3): 251256.

GABARD, L.; RUSSI, I. 2005. Efecto de la intensidad de pastoreo en producción y dinámica poblacional de Sorghum sudanense var. Comiray. Tesis Ingeniero Agrónomo. Montevideo, Uruguay. Facultad de Agronomía. 100 p.

GIORELLO, D.; JAURENA, M.; BOGGIANO, P.; PÉREZ GOMAR, E. 2012. Respuesta al riego suplementario en pasturas y forrajes. En: Seminario internacional, riego en cultivos y pasturas, 2., Salto, Uruguay. Montevideo: INIA. p. 45 - 54.

GORDON, I. 2000. Plant-animal interactions in complex plant communities: from mechanism to modelling. En: Grassland ecophysiology and grazing ecology. Cambridge: CABI Publishing. p. 191-207.

HOLLAND, C.; KEZAR, W. 1990. Pioneer forage manual: A nutritional guide. Pioneer HiBred International. $55 \mathrm{p}$.

JAMIESON, W.; HODGSON, J. 1979. The effect of daily herbage allowance and sward characteristics upon the ingestive behaviour and herbage intake of calves under strip grazing management. Grass and Forage Science, 34: 261-271. 
KJELDAL, J. 1984. Official methods of analysis. Washington, D. C.: Association of Official Agricultural Chemists.

LUZARDO, S.; CUADRO, R.; LAGOMARSINO, X.; BRITO, G.; LA MANNA, A. 2014. Tecnologías para la intensificación de la recría bovina en el basalto - suplementación infrecuente sobre campo natural y pasturas mejoradas en basalto. En: Berretta, E.J.; Montossi, F.; Brito, G. (eds.). Alternativas tecnológicas para los sistemas ganaderos del Basalto. Montevideo: INIA. p. 93-125. (Serie Técnica; 217).

MONTOSSI, F. 1996. Comparative studies of the implications of condensed tannins in the evaluation of Holcus lanatus and Lolium spp. swards for sheep performance. PhD. Thesis. Massey University. New Zealand. $228 \mathrm{p}$.

MONTOSSI, F.; PIGURINA, G.; SANTAMARINA, I.; BERRETTA, E. 2000. Selectividad animal y valor nutritivo de la dieta de ovinos y vacunos en sistemas ganaderos: teoría y práctica, INIA Tacuarembó. Montevideo: INIA. 84 p. (Serie Técnica; 113).

MONTOSSI, F.; SOARES DE LIMA, J. M.; BRITO, G.; BERRETTA, E. 2014. Impacto en lo productivo y económico de las diferentes orientaciones productivas y tecnológicas propuestas para la región de Basalto. En: Berretta, E.J.; Montossi, F.; Brito, G. (eds.). Alternativas tecnológicas para los sistemas ganaderos del Basalto. Montevideo: INIA p. 557-568 (Serie Técnica; 217).

NICOL, A.; BROOKES, A. 2007. The metabolisible energy requirements of grazing livestock. En: Rattray, P.V.; Brookes, I.M.; Nicol, A.M. (eds.). Pasture and supplements for grazing animals. Hamilton: New Zealand Society of Animal Production. p. 151-172. (Occasional publication; 14).

PIGURINA, G.; METHOL, M. 2004. Anexo I. Recopilación de resultados de análisis efectuados en el laboratorio nutrición animal de la Estación Experimental Alberto Böerger, INIA La Estanzuela, Años 19721985. En: Mieres, J.M. (ed.). Guía para la alimentación de rumiantes, INIA La Estanzuela. Montevideo: INIA. p. 7-8. (Serie Técnica; 142).
PORDOMINGO, A. 2003. Suplementación con granos a bovinos en pastoreo. [En línea]. Consultado abr. 2015. Disponible en: http:/ /www.produccion-animal.com.ar/ informacion_tecnica/suplementacion/67suplementacion_con_granos en_pastoreo.pdf

RELLING, A. E.; MATIOLLI, G. A. 2003. Fisiología digestiva y metabólica de los rumiantes. La Plata: EDULP. 72 p.

ROVIRA, P. 2002. Efecto de la sombra artificial en el engorde de novillos durante los meses de verano. En: Jornada anual de producción animal. Resultados experimentales, INIA Treinta y Tres. Montevideo: INIA. p. 79-95. (Serie Actividades de Difusión; 294).

ROVIRA, P.; ECHEVERRÍA, J. 2013. Desempeño productivo de novillos pastoreando sudangras o sorgo forrajero nervadura marrón (BMR) durante el verano. Revista Veterinaria, 24 (2): 91-96.

ROVIRA, P.; ECHEVERRÍA, J. 2014. Desempeño productivo de novillos pastoreando sudangrás o sorgo forrajero nervadura marrón (BMR) durante el verano. En: Estrategias de intensificación ganadera, INIA Treinta y Tres. Montevideo: INIA. p. 59-65. (Serie Actividades Difusión; 734).

SAS INSTITUTE INC. 2013. SAS/STAT 9.4 User's Guide. Cary: SAS Institute Inc.

SILUNGWE, D. 2011. Evaluation of forage yield and quality of sorghum, sudangrass and pearl millet cultivars in Manawatu. MSc. Thesis, Massey (NZ), Massey University. $140 \mathrm{p}$.

SIMEONE, A.; BERETTA, V. 2004. Uso de alimentos concentrados en sistemas ganaderos: ¿Es buen negocio suplementar al ganado? En: Jornada anual de la Unidad de Producción Intensiva de Carne: Manejo nutricional en ganado de carne. Montevideo: Facultad de Agronomía. EEMAC. p. 10-17.

VAN SOEST, J. 1982. Nutritional ecology of the ruminant. Ithaca, New York: Cornell University Press. p. 23-38.

VAZ MARTINS, D.; SEIGAL, E.; PITTALUGA, 0. 2003. Producción de carne con sudangrass dulce, híbrido de sudangrass por sorgo granífero y sorgo doble propósito. En: Vaz Martins, D. (eds.). Avances sobre engorde de novillos en forma in- 
tensiva, INIA Tacuarembó. Montevideo: INIA. p. 19-22. (Serie Técnica; 135).

WADE, M.; CARVALHO, P. 2000. Defoliation patterns and herbage intake on pastures. En: Lemaire, G.; Hodgson, J.; De Moraes, A.; Carvalho, P.C. de F.; Nabinger, C. (eds.). Grassland ecophysiology and grazing ecology. Oxfordshire: Cab International. p. 233248.
WILLAMSON, J.; BLAIR, H.; GARRICK, D.; POMROY, W.; P. DOUCH. 1994. The relationship between internal parasite burden, faecal egg count, and mucosal mast cells in fleeceweight-selected and control sheep. Proc. New Zeal. Soc. An., 54: 9-13. 
1. INTEGRACIÓN DE RESULTADOS DE ENSAYOS NACIONALES DE RECRÍA Y ENGORDE DE NOVILLOS SOBRE SORGOS FORRAJEROS

Para poder integrar la información presentada en esta publicación con la investigación previa realizada a nivel nacional, se analizaron los promedios de las performances animales individuales (GMD) de cada ensayo y se resume en la Figura 1 (información ampliada en Anexo 1).

Las ganancias registradas sobre estos verdeos de verano oscilan en un rango aproxi- mado de 0,250 a 1,200 kg/an/día. Al discriminar la información entre aquellas situaciones sin suplementación y aquellas con animales suplementadas, no se detecta una gran diferencia en las ganancias promedio $(0,600$ vs. $0,700 \mathrm{~kg} / \mathrm{an} /$ día para animales sin suplementar y suplementados, respectivamente), salvo por un valor máximo en animales suplementados logrado en un período corto de tiempo coincidente con el momento de mayor productividad y calidad del cultivo.

Por otro lado, el Cuadro 1 presenta los promedios y rangos de las cargas animales manejadas en promedio para cada una de las experiencias de investigación nacionales.

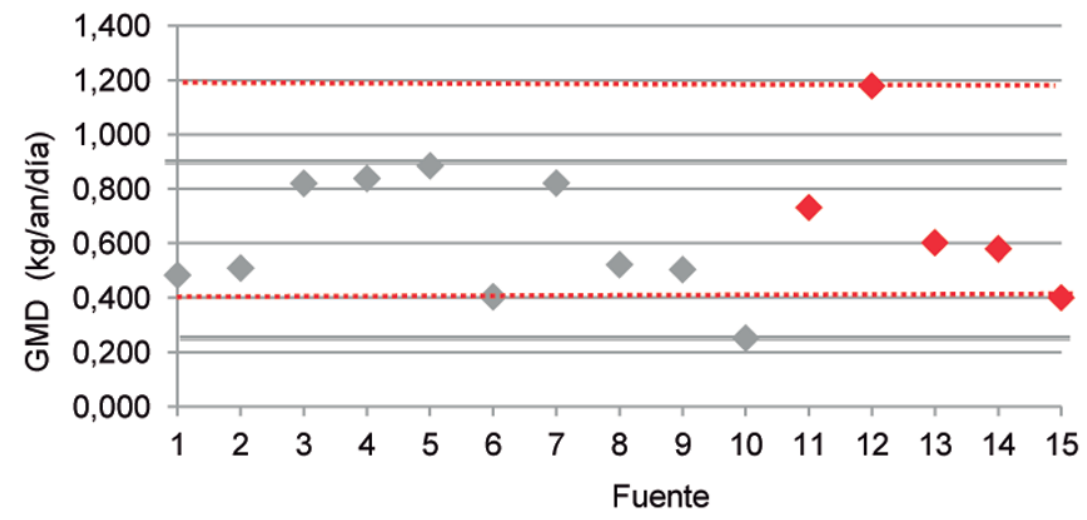

- sin suplementar $\quad$ suplementados

Nota: Correspondencia entre número en la gráfica y fuente: ver Anexo 1.

Figura 1. Ganancia media diaria promedio de trabajos de investigación nacionales de recria y engorde de novillos sobre sorgos forrajeros (con y sin suplementación).

\footnotetext{
*Ing. Agr. MSc. Programa Nacional de Producción de Carne y Lana, INIA Tacuarembó.

** Ing. Agr. PhD. Director Nacional de INIA.

*** Ing. Agr. Programa Nacional de Producción de Carne y Lana, INIA Tacuarembó (2011-2015).

**** Ing. Agr. PhD. Programa Nacional de Producción de Carne y Lana, INIA Tacuarembó.

***** Ing. Agr. Programa Nacional de Producción de Carne y Lana, INIA Tacuarembó.
} 
Cuadro 1. Carga animal promedio de trabajos de investigación sobre verdeos de verano (con y sin suplementación).

\begin{tabular}{|ccc|}
\hline Suplementación & \multicolumn{2}{c|}{$\begin{array}{c}\text { Carga Animal } \\
\text { (UG/ha promedio) }\end{array}$} \\
\hline \multirow{3}{*}{ Sin } & Promedio & 5,7 \\
& Máximo & 6,6 \\
& Mínimo & 4,9 \\
\hline \multirow{3}{*}{ Con } & Promedio & 6,0 \\
& Máximo & 6,6 \\
& Mínimo & 5,6 \\
\hline
\end{tabular}

1 UG $=380 \mathrm{~kg}$ de PV (peso vivo).

A pesar de una leve tendencia superior en dotación animal para los ensayos sobre estos verdeos de verano suplementados (promedio y mínimo), estas diferencias no son de gran magnitud.

Para conocer cómo se relaciona la performance individual en términos de ganancia individual (GMD), con la capacidad de carga del sistema en términos de carga animal, se presenta la Figura 2.

No existió una asociación fuerte entre la carga animal promedio y la performance animal individual. Esto probablemente se explique por la elevada variabilidad de las bases forrajeras, siendo $72 \%$ el coeficiente de variación (CV \%) de las disponibilidades de forraje en promedio de los ensayos, lo cual influyó seguramente en las performances individuales, las cuales fueron muy variadas entre ensayos. Complementariamente, la variabilidad de la calidad del forraje ofrecido (CV PC = 112 \%; CV FDN = 26 \%) también contribuye a la variabilidad de la respuesta animal entre ensayos y entre años. Las variaciones en el estrés térmico entre años, entre otros factores, pueden afectar esta asociación.

En cuanto a la alta variabilidad interanual en performance individual, con respecto a las razas utilizadas, de los 15 ensayos analizados, solo uno utilizó la raza Holando y el resto utilizó razas británicas y sus cruzas. Otra fuente de variación podría ser la categoría utilizada, pero igualmente en solo 2 casos de los 15 presentados se trata de categorías "más extremas" en el rango de peso vivo al comenzar los ensayos (animales de menos de $200 \mathrm{~kg}$ iniciales o animales de más de $400 \mathrm{~kg}$ iniciales). En definitiva, las condiciones experimentales en cuanto al componente genético animal y categoría no serían tan relevantes como la variabilidad atribuible al "efecto año" reflejada en la cantidad y calidad del forraje.

Otro elemento adicional a considerar en el análisis es la inclusión de la suplementación (cantidad, fuente, valor nutritivo, etc.). Este factor es evaluado a continuación, separando la base de datos entre los animales que recibieron o no suplementación.

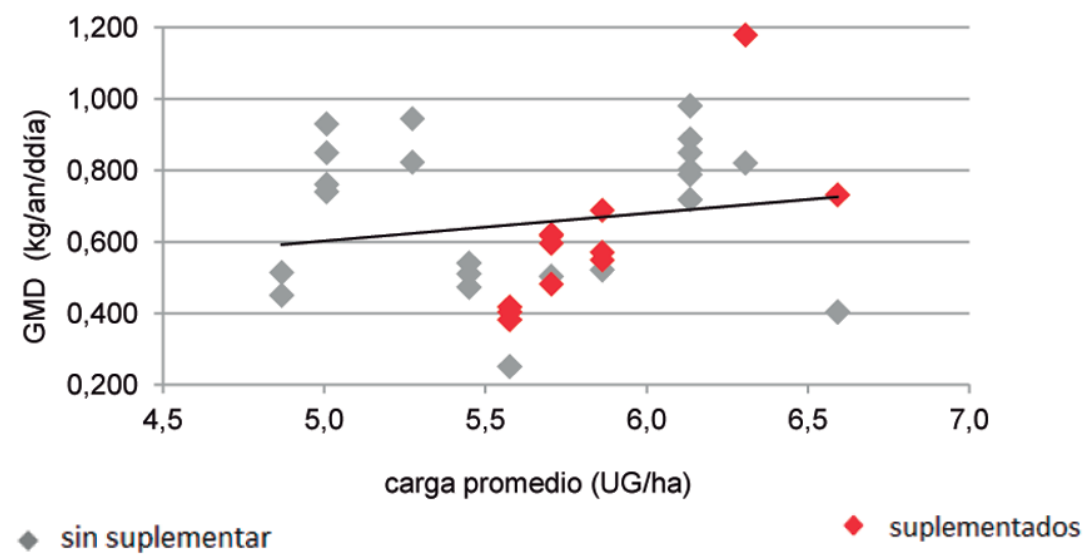

Figura 2. Asociación entre carga animal promedio (UG/ha) y performance animal individual promedio (GMD) de novillos en trabajos de investigación nacionales sobre sorgos forrajeros (con y sin suplementación) (1 UG $=380 \mathrm{~kg}$ de PV). 
a) Sin suplementación

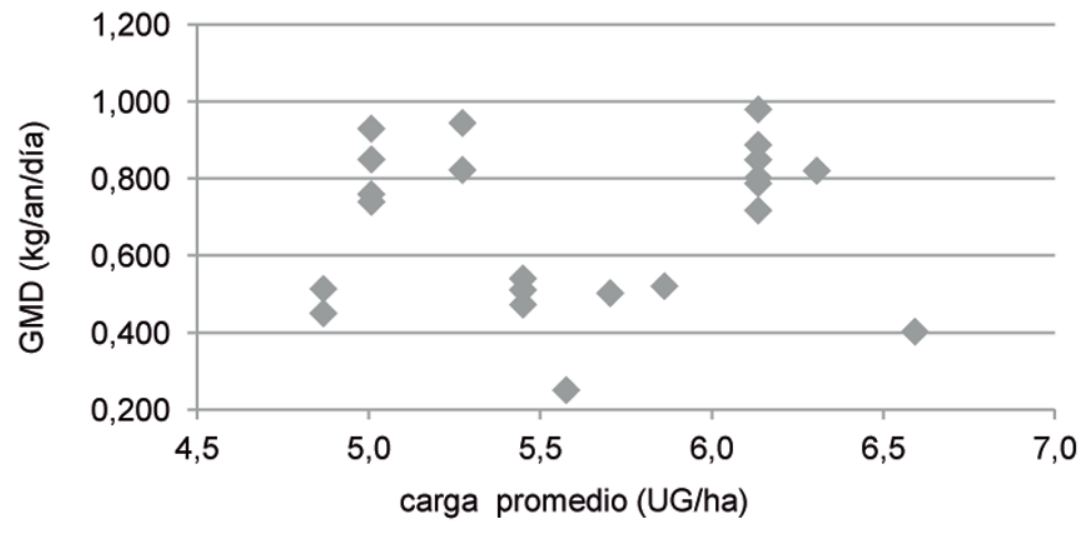

b) Con suplementación

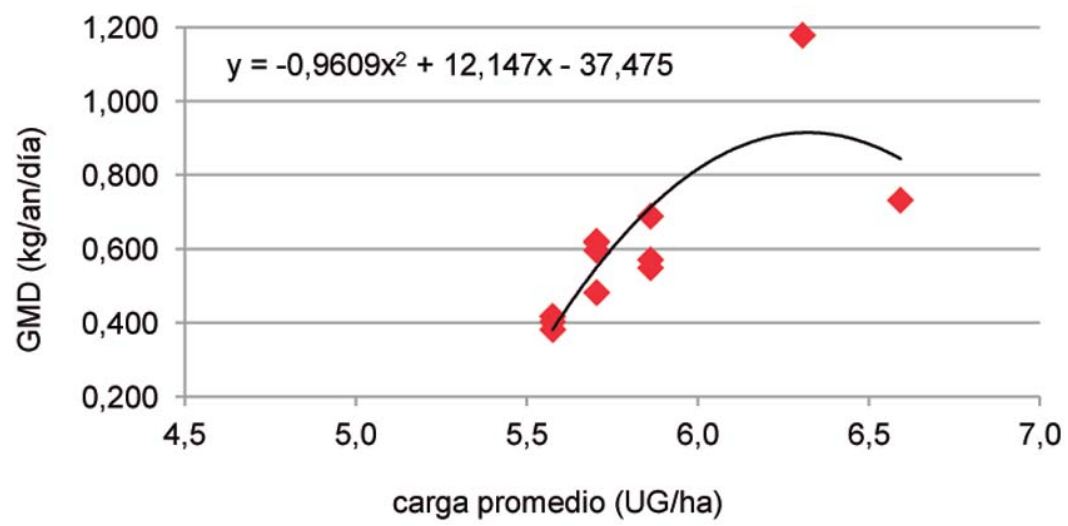

Figura 3. Asociación entre carga animal promedio (UG/ha) y ganancia media diaria (GMD) de novillos en trabajos de investigación nacionales sobre sorgos forrajeros, discriminado por tratamientos no suplementados (a) y suplementados (b).

Si se analiza la misma información, pero separando los casos suplementados de los no suplementados, se obtienen los resultados que se observan en la Figura 3.

No se encontró una asociación entre la carga animal y la ganancia media diaria de los animales que no fueron suplementados. Dado que en este caso la performance individual está explicada por el consumo de forraje exclusivamente, esto se ajusta a las fuentes de variación mencionadas previamente, en cuanto a la elevada variabilidad entre las bases forrajeras de cada ensayo y año de evaluación.

En contraste, se registró una asociación cuadrática entre la carga animal promedio y la performance individual en animales suplementados $\left(R^{2}=0,70\right)$, constatándose que con los datos promedio considerados, una mayor carga determina mayores ganancias individuales hasta un punto $(6,3 \mathrm{UG} / \mathrm{ha})$ en que las ganancias empiezan a disminuir. $\mathrm{Si}$ no se considera el valor extremo de ganancia diaria superior -que se obtuvo con un período de solamente 58 días- el ajuste sigue siendo cuadrático $\left(R^{2}=0,71\right)$. Cuando solo se consideran asignaciones de suplemento al 0,5\% PV, el ajuste mejora y sigue siendo cuadrático $\left(R^{2}=0,75\right)$, si bien la carga animal no debería superar 5,8 UG/ha para no afectar la ganancia individual. Este tipo de ajuste concuerda con lo reportado por Riewe (1984), quien menciona un rango óptimo teórico de carga animal en pastoreo en donde la performance individual va descendiendo mientras que la producción por unidad de superficie tiene un comportamiento cuadrático a medida que aumenta la carga animal. 
Una mala calidad del forraje de los verdeos de verano está asociada al manejo de la fase reproductiva de estos cultivos -encañazónla cual aumenta normalmente con el uso de cargas animales bajas (Carámbula, 2007).

Por ende, extremos muy bajos de carga animal promedio/ha no presentan performances individuales óptimas como lo reporta Riewe (1984).

La suplementación implica, por lo general, un cierto nivel de sustitución del consumo de forraje por suplemento (Holmes, 1987). Esto significa que la ganancia de peso vivo de los animales suplementados está explicada por la suma del consumo de forraje más el suplemento, por lo cual la incidencia del consumo proveniente del forraje en la ganancia es menor que en los casos de animales no suplementados. Tomando en cuenta lo que ya se discutió en cuanto a la elevada variabilidad en cantidad y calidad del cultivo de sorgo (efecto año), los animales suplementados cuentan con un consumo mucho menos variable proveniente del suplemento y eso explicaría la mejor asociación encontrada entre la carga animal y la ganancia individual de los novillos (Figura 3). Por lo tanto, la incorporación de la suplementación resulta en una mayor predictibilidad y estabilidad en la producción animal de novillos en crecimiento sobre esta base forrajera (sorgo forrajero) en las condiciones estivales del Uruguay.
Por último, se evaluó la asociación entre la carga animal utilizada en cada caso en función de la productividad lograda por unidad de superficie, para conocer el impacto que tiene una variable sobre la otra (Figura 4).

Al presentar toda la información conjuntamente (suplementados y no suplementados), si bien el coeficiente de determinación obtenido es bajo $\left(R^{2}=0,28\right)$, existe una asociación cuadrática que determina que en el rango considerado, cargas mayores resultan en producciones de PV por hectárea mayores. Para conocer si la suplementación podría tener un efecto en la asociación entre la carga y la productividad animal por unidad de superficie, se evaluó su asociación por separado estas dos variables (Figura 5).

Los tratamientos de los ensayos sin suplementación representan la mayoría de la información y aún así, nuevamente presenta un coeficiente de determinación bajo $\left(R^{2}=\right.$ $0,23)$. Al evaluar solamente los tratamientos suplementados, dicho coeficiente es marcadamente superior $\left(R^{2}=0,73\right)$, determinando la existencia de una asociación cuadrática y positiva entre la carga animal promedio y productividad de peso vivo/ha, donde a partir de una carga de 6,4 UG/ha, la asociación se transforma en negativa. Nuevamente, se destacan los argumentos esgrimidos con respecto a la ventaja de la inclusión de la su-

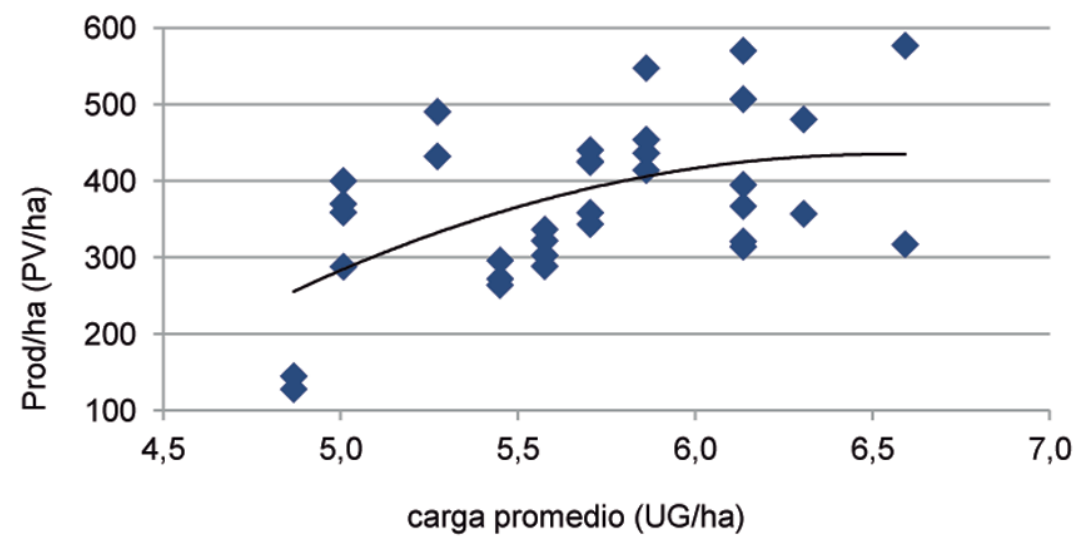

1 UG = $380 \mathrm{~kg}$ de PV (peso vivo); datos con y sin suplementación juntos, sin discriminar.

Figura 4. Asociación entre carga animal promedio (UG/ha) y productividad por unidad de superficie (PV/ha) de novillos en trabajos de investigación nacionales sobre sorgos forrajeros (con y sin suplementación). 
a) Sin suplementación

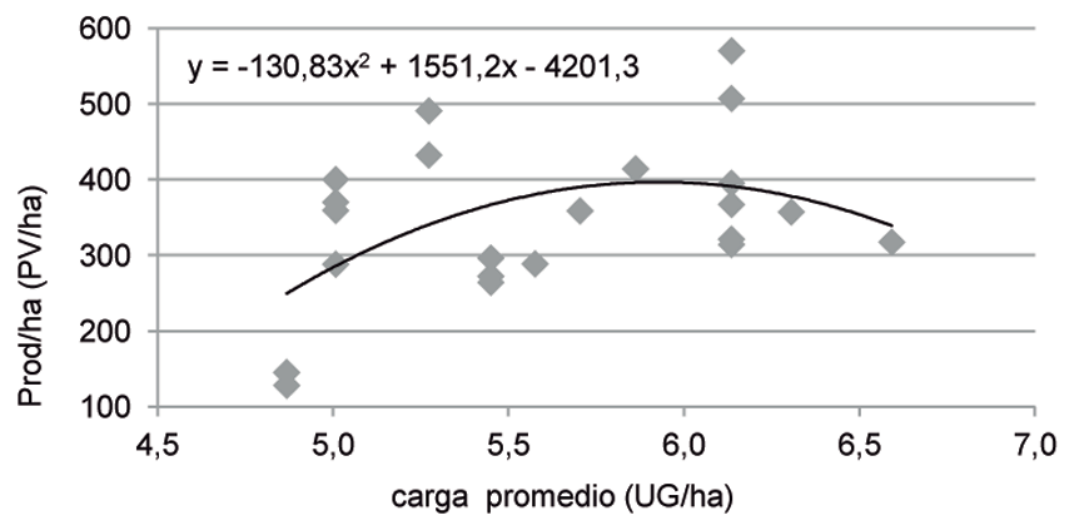

b) Con suplementación

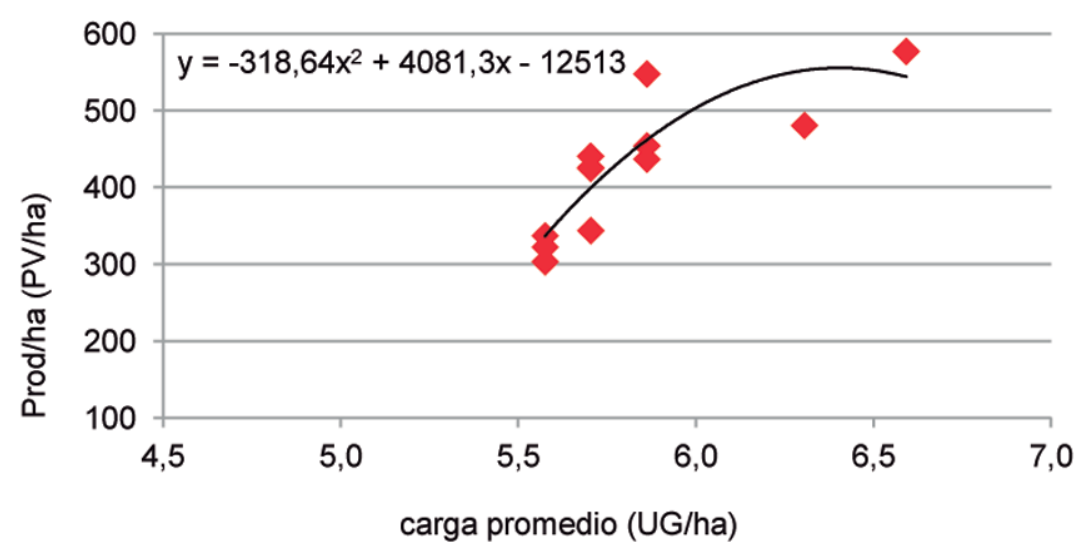

Figura 5. Asociación entre carga animal promedio (UG/ha) y productividad animal por unidad de superficie ( $\mathrm{PV} / \mathrm{ha}$ ) de novillos en trabajos de investigación nacionales sobre sorgos forrajeros, discriminado por tratamientos no suplementados (a) y suplementados (b) (1 UG = $380 \mathrm{~kg}$ de PV).

plementación para aumentar la producción y estabilidad de la misma en estos sistemas de recría y engorde de novillos.

\section{IMPLICANCIAS Y}

\section{RECOMENDACIONES}

PRÁCTICAS DE ESTA LÍNEA DE TRABAJO DE INVESTIGACIÓN

Para explorar las productividades de forraje registradas en los estudios presentados en la presente publicación, es necesario un adecuado manejo agronómico del verdeo de sorgo forrajero, particularmente asegurándose una fecha temprana de siembra, lo cual es fundamental para lograr un mayor período utilización del mismo y así lograr buenos resultados productivos a nivel de la recría y engorde de novillos de sobre año. Las opciones forrajeras presentadas (sudangrás y sorgo) se adaptan a las condiciones agroecológicas de la región del Basalto.

La planificación de la siembra de los sorgos forrajeros debe comenzar con una adecuada preparación desde la etapa de barbecho. Esto posibilita un control adecuado de malezas así como también una mayor acumulación de agua en el perfil suelo, lo cual es clave en las etapas tempranas del cultivo. La fecha de siembra de este verdeo va a estar condicionada por la temperatura del 
suelo que debe ser de al menos 17 o $18^{\circ} \mathrm{C}$, tres días consecutivos antes de la siembra.

La densidad de siembra depende de factores inherentes a la calidad de la semilla (peso específico, \% de germinación, pureza y vigor). De cualquier forma, como recomendación técnica debe considerarse ciertos rangos de siembra entre $16-20 \mathrm{~kg} / \mathrm{ha}$, si se utilizan semillas de buena calidad. Las semillas deben ubicarse a una profundidad de siembra cercana a los $2-3 \mathrm{~cm}$.

El potencial productivo de forraje de sudangrás y sorgos forrajeros bien implantados, y con un paquete de manejo agronómico acorde al ambiente ecológico donde se desarrollan, es generalmente muy alto. El manejo de la fertilización de cultivos de verano se basa fundamentalmente en análisis del nivel nutritivo del suelo, con el fin de evaluar la capacidad de aporte de nutrientes claves del suelo para el cultivo. Por este motivo, este debe comenzar con un correcto muestreo de suelo para tener en cuenta las disponibilidades de nutrientes y adecuarla a las necesidades del cultivo. Los requerimientos de estos cultivos de fósforo en el suelo se ubican en los 16 ppm de P (Bray I). Las unidades de nitrógeno a la siembra van a estar condicionadas en función del manejo del cultivo antecesor, así como también de la etapa de barbecho. De igual manera, hay que tener en cuenta que tanto el sudangrás como el sorgo son especies de alta respuesta en materia seca a la fertilización nitrogenada, si las condiciones ambientales permiten expresar dicho potencial. Esta alta producción de forraje no siempre se traduce en altas productividades de peso vivo por hectárea debido principalmente a la baja utilización que se logra del forraje a nivel comercial. Este último aspecto es muy importante a tener en cuenta a la hora de planificar la fecha de las diferentes áreas de siembra, de manera de asegurar que una vez que los cultivos estén aptos para ser pastoreados, se cuente con el suficiente número de animales y divisiones, entre y a nivel de los potreros, como para hacer una correcta utilización del forraje.

Estratégicamente, el uso de esta base forrajera durante los meses estivales podrían tener un impacto importante en la producción y estabilidad del sistema global de producción ganadera, ya que su uso permitiría el descanso o disminución de carga animal sobre las praderas permanentes que pudieran estar co-existiendo en el mismo. De esta forma, las praderas aumentarían la chance de superar exitosamente el estrés normal del verano, en cuanto a eventuales déficits hídricos y/o temperaturas excesivas, contribuyendo así a su persistencia y productividad.

La disponibilidad de forraje, su composición botánica y valor nutritivo explican las restricciones de esta alternativa forrajera -como única fuente de nutrientes- para las condiciones del Basalto durante el período estival para potencializar la expresión productiva de novillos con alta demanda de nutrientes. Estos parámetros -usando estos verdeos de verano como única dieta- indican la limitante del uso de cargas superiores a 7 novillos/ha para alcanzar pesos superiores a los $300 \mathrm{~kg} \mathrm{PV} \mathrm{a} \mathrm{la}$ salida del verano.

Los sorgos forrajeros cuando son utilizados con novillos en activo crecimiento, con importantes demandas de energía y proteína, permiten lograr ganancias superiores a los 0,400-0,600 kg/an/día con una suplementación estratégica en el rango del 0,5-1,0 \% del PV. En estas condiciones, la producción animal por unidad de superficie supera los $450 \mathrm{~kg} \mathrm{PV/ha} \mathrm{en} \mathrm{un} \mathrm{período} \mathrm{aproximado} \mathrm{de}$ 100 días de utilización del sorgo. El beneficio obtenido por la suplementación sobre los animales se observa particularmente en etapas muy avanzadas del cultivo cuando este comienza a perder valor nutricional, donde la respuesta a la suplementación mejora. Posiblemente, la respuesta productiva y económica mejoraría si la aplicación de esta suplementación estratégica se concentrara en esta "ventana" de oportunidad.

En un contexto de una recría acelerada (novillo de 15 meses con $300 \mathrm{~kg}$ ), la implementación del uso de una alimentación estratégica de verano con sorgos de pastoreo a alta carga permiten intensificar el sistema productivo, logrando una disminución de la edad de faena y la calidad del producto (ca- 
nal y carne), aumentando la producción de carne por superficie, por lo tanto, haciendo más eficiente todo el sistema de producción ganadero.

En un contexto de ciclo completo, lo mencionado en el punto anterior, sumado a una posterior buena alimentación -7 meses desde principios de otoño a fin de primavera- en base a pasturas mejoradas combinado con el uso de suplementos, podría permitir terminar los novillos con $500-520 \mathrm{~kg}$ a una edad inferior a los 30 meses. Siguiendo este esquema, no sería necesario implementar una alimentación estival mejorada una vez más, ya que los animales se irían antes del sistema. Consecuentemente, los costos del predio se verían controlados y el balance forrajero global se vería mejorado.

En un contexto de ciclo incompleto, una opción válida a la hora de planificar las actividades del predio sería considerar la venta de los novillos recriados en el verdeo -con o sin suplementación-dependiendo del PV final a la salida del verano.

\section{CONCLUSIONES GENERALES}

Los cultivos forrajeros anuales estivales, como lo son el sudangrás o el sorgo forrajero, son las alternativas forrajeras que necesitan de una mayor inversión económica relativa en comparación con otras opciones forrajeras para los esquemas de producción animal sobre base pastoril. Esto es compensado por su muy elevada producción de forraje en un período de uso relativamente corto de tiempo, y particularmente en el período estival, donde las alternativas son muy escasas. Ello requiere aún mayor relevancia en suelos medios a profundos de la región del Basalto durante el verano. Para poder recuperar la inversión, el productor debe tener la seguridad de que espera obtener altas producciones individuales pero, sobre todo, altas producciones de peso vivo por hectárea en los períodos considerados.
Según los resultados obtenidos en los ensayos de esta publicación y la combinación de estos con los antecedentes nacionales, la asociación entre carga y productividad individual no es tan clara si no se considera la suplementación en la implementación de esta tecnología. Existe una alta variabilidad en la productividad individual y por unidad de superficie en esquemas de recría-engorde de ganado de carne sobre estas bases forrajeras no suplementadas, lo que hace difícil predecir los resultados físicos de la actividad, y por tanto económicos, en relación a la carga animal. Al incluir la suplementación en el esquema de verano sobre estos sorgos, la asociación entre la carga animal y productividad animal mejora, facilitando las proyecciones de producción y mejorando la estabilidad del sistema productivo.

Por otro lado, a los costos de implantación de estos verdeos de sorgos se deben agregar los costos de suplementación, es por ello que es clave la aplicación de un paquete tecnológico integral que facilite y que mejore la certidumbre del cumplimento de los objetivos productivos, de manera de minimizar el riesgo financieroeconómico que implica la inversión de esta propuesta. Aún con determinados escenarios muy favorables de relaciones de precios de los insumos y productos, el productor y/o su asesor deberían realizar la evaluación económica de esta opción en varios escenarios de precios y productivos. En este sentido, el INIA con esta publicación aporta coeficientes técnicos que pueden ayudar al proceso de toma de decisiones en cuanto a la conveniencia o no de implementar esta tecnología en predios ganaderos extensivos o semi-extensivos, particularmente del Basalto. Sobre esta base, se recomienda que la decisión de implementar esta tecnología en los predios ganaderos siempre considere no solo el beneficio puntual de realizar esta actividad (margen bruto o neto/ha), si no también poner atención especialmente en el impacto que la tecnología tenga en todo el sistema (ingreso de capital en el predio). 


\section{BIBLIOGRAFÍA}

BERLANGERI, S. 2008. Efecto del manejo y el material genético en la productividad de sorgo forrajero bajo pastoreo. Tesis Ingeniero Agrónomo. Montevideo, Uruguay. Facultad de Agronomía. 189 p.

CARÁMBULA, M. 2007. Verdeos de verano. Montevideo: Hemisferio Sur. 226 p.

ESQUIVEL, J.; VELAZCO, J.; ROVIRA, P. 2006. Efecto del acceso a sombra artificial en la ganancia de peso, estrés y conducta de novillos pastoreando sudangrás durante el verano. En: Jornada Anual Unidad Experimental Palo a Pique, INIA Treinta y Tres. Montevideo: INIA. p 2236. (Serie Actividades de Difusión; 511).

HOLMES, C. 1987. Pastures for dairy cows. En: Nicol, A.M. (ed.). Livestock feeding on pasture. Hamilton: NZSAP. p. 113-145. (Occasional Publication; 10).

QUINCKE, A; GARCÍA LAMOTHE, L.; MORÓN, A. 2008. Manejo de la fertilización en cultivos de verano. En: Jornada INIA La Estanzuela. Cultivos de verano, INIA La Estanzuela. Montevideo: INIA. p. 1-4. (Serie Actividades de Difusión; 550).
RIEWE, M. 1984. Manejo del pastoreo fijo o variable en la evaluación de pasturas. En: Lascano, C.; Pizarro, E. (eds). Evaluación de pasturas con animales: alternativas metodológicas. Memorias de una reunión de trabajo celebrada en Perú, 15 de octubre, 1984. Cali: CIAT. p 61-84.

ROVIRA, P. 2002. Efecto de la sombra artificial en el engorde de novillos durante los meses de verano. En: Jornada anual de producción animal. Resultados experimentales, INIA Treinta y Tres. Montevideo: INIA. p. 79-95. (Serie Actividades de Difusión; 294).

ROVIRA, P.; ECHEVERRÍA, J. 2013. Desempeño productivo de novillos pastoreando sudangras o sorgo forrajero nervadura marrón (BMR) durante el verano. Revista Veterinaria, 24 (2): 91-96.

VAZ MARTINS, D.; SEIGAL, E.; PITTALUGA, 0. 2003. Producción de carne con sudangrass dulce, híbrido de sudangrass por sorgo granífero y sorgo doble propósito. En: Vaz Martins, D. (eds.). Avances sobre engorde de novillos en forma intensiva, INIA Tacuarembó. Montevideo: INIA. p. 19-22. (Serie Técnica; 135). 


\section{ANEXOS}

\section{ANEXO I}

Resumen de resultados de investigación nacionales sobre pastoreo de verdeos de verano con y sin suplementación.

\begin{tabular}{|c|c|c|c|c|c|c|c|}
\hline $\mathbf{n}$ & Fuente & Trat. & BF & PVi & PV & gmd & Días \\
\hline \multicolumn{8}{|c|}{ SIN SUPLEMENTACIÓN } \\
\hline \multirow{3}{*}{$r$} & \multirow{3}{*}{ 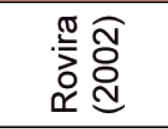 } & \multicolumn{2}{|c|}{ Lomadas del Este } & \multirow{3}{*}{401} & & \multirow[b]{2}{*}{0,451} & \multirow{3}{*}{53} \\
\hline & & Sin sombra & $\mathrm{Sd}$ & & 424 & & \\
\hline & & Con sombra & Sd & & 429 & 0,514 & \\
\hline \multirow{4}{*}{$N$} & \multirow{4}{*}{ 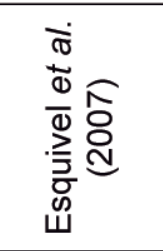 } & \multicolumn{2}{|c|}{ Lomadas del Este } & \multirow{4}{*}{278} & & & \multirow{4}{*}{68} \\
\hline & & Sin sombra & $\mathrm{Sd}$ & & 312 & 0,473 & \\
\hline & & $\begin{array}{l}\text { Sombra } \\
\text { restringida }\end{array}$ & Sd & & 312 & 0,511 & \\
\hline & & $\begin{array}{l}\text { Con sombra } \\
\text { libre acceso }\end{array}$ & Sd & & 317 & 0,541 & \\
\hline \multirow{5}{*}{$m$} & \multirow{5}{*}{ 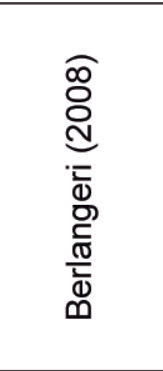 } & \multicolumn{2}{|c|}{ Suelos del Litoral Norte } & \multirow{5}{*}{333} & & \multirow{5}{*}{90} \\
\hline & & $\begin{array}{c}\text { Sorgo BMR con } \\
\text { rotativa }\end{array}$ & $\mathrm{Sg}$ & & 417 & 0,930 & \\
\hline & & $\begin{array}{l}\text { Sorgo BMR sin } \\
\text { rotariva }\end{array}$ & $\mathrm{Sg}$ & & \multicolumn{2}{|r|}{0,760} & \\
\hline & & $\begin{array}{l}\text { Sorgo no BMR } \\
\text { con rotativa }\end{array}$ & $\mathrm{Sg}$ & & \multicolumn{2}{|r|}{0,850} & \\
\hline & & $\begin{array}{l}\text { Sorgo no BMR } \\
\text { sin rotativa }\end{array}$ & $\mathrm{Sg}$ & & \multicolumn{2}{|l|}{400} & \\
\hline & & Suelos del Lito & Sur & & & & \\
\hline & & Sudan $60 \mathrm{~cm}$ & $\mathrm{Sd}$ & & 290 & 0,888 & \\
\hline & in & $\mathrm{Sg} \mathrm{H} 160 \mathrm{~cm}$ & $\mathrm{Sg}$ & & 270 & 0,849 & \\
\hline Q & 资 & $\mathrm{Sg} \mathrm{H} 260 \mathrm{~cm}$ & $\mathrm{Sg}$ & 192 & 277 & 0,980 & $82-$ \\
\hline & $\sum^{\pi} \mathbb{N}$ & Sudan $100 \mathrm{~cm}$ & $\mathrm{Sd}$ & & 263 & 0,718 & \\
\hline & $N$ & $\mathrm{Sg} \mathrm{H} 1100 \mathrm{~cm}$ & $\mathrm{Sg}$ & & 266 & 0,788 & \\
\hline & & $\mathrm{Sq} \mathrm{H} 2100 \mathrm{~cm}$ & $\mathrm{Sq}$ & & 255 & 0,803 & \\
\hline & 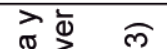 & Lomadas del & & & & & \\
\hline ما & $\frac{\pi}{3} \frac{\pi}{2} . \frac{\pi}{6}$ & Sudan & $\mathrm{Sd}$ & 304 & 378 & 0,823 & 73 \\
\hline & 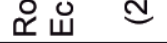 & Sorgo & $\mathrm{Sg}$ & & 388 & 0,945 & \\
\hline & $-r$ & Basalto & & & & & \\
\hline 0 & $\frac{2}{x} \div$ & Sudan & Sd & 313 & & & 105 \\
\hline & 崖 & Sorgo & $\mathrm{Sg}$ & & 355 & 0,403 & \\
\hline & $-N$ & Basalto & & & & & \\
\hline$\Lambda$ & $\frac{0}{x}, 0$ & Sudan & Sd & 296 & & & 58 \\
\hline & 崖完 & Sorgo & $\mathrm{Sq}$ & & 343 & 0,821 & \\
\hline & సু ঃ _ & Basalto & & & & & \\
\hline$\infty$ & 离哭- & Sorgo & $\mathrm{Sq}$ & 269 & 325 & 0.521 & 106 \\
\hline ه & ㄸำ & Basalto & & 265 & & & of \\
\hline क) & 离选 & Sorgo & $\mathrm{Sg}$ & 265 & 313 & 0,503 & 96 \\
\hline 0 & ח్ & Basalto & & 207 & & & 05 \\
\hline$F$ & 岦 & Sorgo & $\mathrm{Sg}$ & 231 & 261 & 0,251 & 95 \\
\hline
\end{tabular}




\begin{tabular}{|c|c|c|c|c|c|c|c|}
\hline $\mathbf{n}$ & Fuente & Trat. & BF & PVi & PVf & gmd & Días \\
\hline \multicolumn{8}{|c|}{ CON SUPLEMENTACIÓN } \\
\hline \multirow[b]{2}{*}{$\mp$} & \multirow{2}{*}{$\begin{array}{l}\overline{\frac{\alpha}{x}} \\
\text { 道 }\end{array}$} & Basal & & \multirow[b]{2}{*}{313} & & & \multirow[b]{2}{*}{105} \\
\hline & & $\begin{array}{c}\text { Suplemento } \\
\text { Sg/Sd }\end{array}$ & $\mathrm{Sg} / \mathrm{Sd}$ & & 391 & 0,732 & \\
\hline \multirow[b]{2}{*}{$\stackrel{N}{\sim}$} & \multirow{2}{*}{ 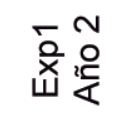 } & Basal & & \multirow[b]{2}{*}{296} & & & \multirow[b]{2}{*}{58} \\
\hline & & $\begin{array}{c}\text { Suplemento } \\
\mathrm{Sg} / \mathrm{Sd}\end{array}$ & $\mathrm{Sg} / \mathrm{Sd}$ & & 272 & 1,179 & \\
\hline \multirow{4}{*}{$m$} & \multirow{4}{*}{ 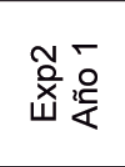 } & \multicolumn{2}{|c|}{ Basalto } & \multirow{4}{*}{269} & & & \multirow{4}{*}{106} \\
\hline & & EG & \multirow{3}{*}{$\mathrm{Sg}$} & & 327 & 0,549 & \\
\hline & & AA & & & 330 & 0,571 & \\
\hline & & GM & & & 341 & 0,689 & \\
\hline \multirow{5}{*}{$\stackrel{ \pm}{\leftarrow}$} & \multirow{5}{*}{ 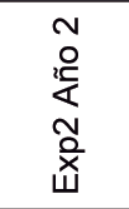 } & \multicolumn{2}{|c|}{ Basalto } & \multirow{5}{*}{265} & & & \multirow{5}{*}{96} \\
\hline & & ES & \multirow{4}{*}{$\mathrm{Sg}$} & & 234 & 0,618 & \\
\hline & & EG & & & 324 & 0,621 & \\
\hline & & AA & & & 322 & 0,596 & \\
\hline & & GM & & & 317 & 0,482 & \\
\hline \multirow{4}{*}{$\stackrel{2}{2}$} & \multirow{4}{*}{ 啇 } & \multicolumn{2}{|c|}{ Basalto } & \multirow{4}{*}{237} & & & \multirow{4}{*}{95} \\
\hline & & EG & \multirow{3}{*}{$\mathrm{Sg}$} & & 273 & 0,382 & \\
\hline & & AA & & & 275 & 0,403 & \\
\hline & & GM & & & 277 & 0,418 & \\
\hline
\end{tabular}

Sd: sudangrás; Sg: sorgo; ES: expeller soja; EG: expeller sorgo; AA: afrechillo arroz; GM: grano maíz; gmd: ganancia media diaria en kg/an/día 
Impreso en Editorial Hemisferio Sur S.R.L.

Buenos Aires 335

Montevideo - Uruguay

Depósito Legal 369.600 
INIA Dirección Nacional

Andes 1365, P. 12

Montevideo

Tel.: 59829020550

Fax: 59829023633

iniadn@dn.inia.org.uy

INIA La Estanzuela Ruta 50, Km 11

Colonia

Tel.: 59845748000

Fax: 59845748012

iniale@le.inia.org.uy

INIA Las Brujas Ruta 48, Km 10 Canelones

Tel.: 59823677641

Fax: 59823677609 inia_lb@lb.inia.org.uy

INIA Salto Grande Camino al Terrible Salto

Tel.: 59847335156

Fax: 59847329624 inia_sg@sg.inia.org.uy

INIA Tacuarembó Ruta 5, Km 386 Tacuarembó

Tel.: 59846322407

Fax: 59846323969 iniatbo@tb.inia.org.uy

INIA Treinta y Tres Ruta 8, Km 281

Treinta y Tres Tel.: 59844522023 Fax: 59844525701 iniatt@tyt.inia.org.uy

www.inia.uy 